\title{
CLUES: A WEB-BASED LAND USE EXPERT SYSTEM FOR THE WESTERN CAPE
}

\author{
ADRIAAN VAN NIEKERK \\ Dissertation presented for the degree of Doctor of Philosophy \\ at \\ Stellenbosch University
}

Promotor: Prof JH van der Merwe

December 2008 


\section{DECLARATION}

By submitting this dissertation electronically, I declare that the entirety of the work contained therein is my own, original work, that I am the owner of the copyright thereof (unless to the extent explicitly otherwise stated) and that I have not previously in its entirety or in part submitted it for obtaining any qualification.

Date: December 2008

Copyright (C) 2008 Stellenbosch University

All rights reserved 


\section{SUMMARY}

GIS has revolutionized geographic analysis and spatial decision support and has greatly enhanced our understanding of the real world though it's mapping and spatial modelling capabilities. Although GIS software is becoming more powerful, less expensive and more userfriendly, GIS still remains the domain of a selected few who can operate and afford these systems. Since the introduction of web mapping tools such as Google Earth, accessibility to geographic information has escalated. Such tools enable anyone with access to a computer and the Internet to explore geographic data online and produce maps on demand. Web mapping products have, however, a very narrow range of functionality. In contrast to GIS that focuses on spatial data capture, storage, manipulation, analysis and presentation, the function of web mapping tools is to visualize and communicate geographical data. The positive impact of web mapping tools suggests, however, that GIS has not yet developed to a level where anyone can use the technology to support spatial decisions and enhance productivity. A possible solution is to close the functional gap between web mapping tools and GIS to make spatial analysis more accessible, thereby promoting geographical awareness and supporting better spatial decisions.

In this research, a web-based spatial decision support system (SDSS) was developed to demonstrate how the Internet can be used to deliver low-cost, user-friendly and interactive spatial analysis functionality to a wide audience. Although the resulting Cape Land Use Expert System (CLUES) was specifically developed to perform land suitability analysis in the Western Cape, the technology can also be applied to other regions and modified for other applications. CLUES consists of five components: a land unit database (LUD), knowledge base, inference engine, web map service (WMS) and graphical user interface (GUI). The LUD consists of polygons (land units) and attributes (land properties), while the knowledge base stores each user's land use requirement rules. These rules are used by the inference engine to rate the suitability of each land unit in the LUD. The result is then mapped by the WMS and presented to the users as suitability maps. Users can direct the entire analysis through a user-friendly GUI.

The development and demonstration of CLUES exposed several advantages and limitations of current technology and has demonstrated that the Internet offers great opportunities for the deployment of spatial analysis and modelling functionality to a wide audience.

\section{KEY WORDS}

Expert system, geographical information system, Internet, land evaluation, spatial decision support system, suitability analysis, web mapping, Web 


\section{OPSOMMING}

GIS het geografiese analise en die ondersteuning van ruimtelike besluitneming revolusionêr verander en ons begrip van die werklike wêreld aansienlik versterk deur die karterings- en ruimtelike modelleringsvermoëns daarvan. Alhoewel GIS programmatuur kragtiger, goedkoper en meer gebruikersvriendelik raak, bly dit steeds die domein van uitverkorenes wat hierdie stelsels kan bedryf en bekostig. Die bekendstelling van web karteringsgereedskap soos Google Earth het toegang tot geografiese inligting grootliks verbreed. Sulke gereedskap laat enigeen met rekenaar- en die Internettoegang toe om geografiese data aanlyn te ondersoek en kaarte op aanvraag te maak. Web karteringsprodukte het egter ' $n$ baie nou funksionele reikwydte. In teenstellings met GIS, wat fokus op vaslegging, berging, manipulasie, analise en visuele voorstelling van ruimtelike data, is die funksie van web karteringsgereedskap slegs om geografiese data te visualiseer en te kommunikeer. Die positiewe impak van web karteringsgereedskap dui egter daarop dat GIS nog nie tot die vlak onwikkel het waar enigeen toegang tot die tegnologie kan bekom om ruimtelike besluite te steun en produktiwiteit te verbeter nie. ' $n$ Moontlike oplossing is om die funksionele gaping tussen web karteringsgereedskap en GIS uit te skakel en ruimtelike analise meer toeganklik te maak om sodoende geografiese bewustheid te bevorder en ruimtelike besluitneming beter te ondersteun.

In hierdie navorsing is ' $\mathrm{n}$ web-gebaseerde ruimtelike besluitsteunstelsel (RBSS) ontwikkel om te toon hoe die Internet gebruik kan word om lae-koste, gebruikersvriendelike en interaktiewe ruimtelik-analitiese funksionaliteit aan ' $n$ wye gehoor te lewer. Alhoewel die voortvloeiende Cape Land Use Expert System (CLUES) spesifiek vir grondgeskiktheidsanalise in die Wes-Kaap ontwikkel is, kan die tegnologie aangewend word in soortgelyke gebiede of vir ander toepassings aangepas word. CLUES bestaan uit vyf komponente: 'n landeenheid databasis (LED), kennisbasis, afleidingsenjin, web kaartdiens (WKD) en grafiese gebruikerskoppelvlak (GGK). Die LED bestaan uit poligone (landeenhede) en attribute (land eienskappe), terwyl die kennisbasis elke gebruiker se reëls rakende grondgebruikvereistes hou. Die afleidingsenjin gebruik hierdie reëls om die geskiktheid van elke landeenheid in die LED te skaal. Die WKD karteer dan die resultaat en stel dit as geskiktheidskaarte aan die gebruikers voor. Gebruikers kan die hele analise met behulp van die gebruikersvriendelike GGK rig.

Die ontwikkeling en demonstrasie van CLUES het die voordele en beperkinge van die huidige tegnologie blootgelê en kon demonstreer dat die Internet groot geleenthede vir die ontplooiing van ruimtelike analise en modelleringsfunksionaliteit aan 'n wye gehoor inhou. 


\section{TREFWOORDE}

Deskundige stelsel, geografiese inligtingstelsel, Internet, grond/land evaluering, ruimtelike besluitsteunstelsel, geskiktheidsanalise, web kartering, Web 


\section{ACKNOWLEDGEMENTS}

I sincerely thank:

- Helene, my wife, for her support, understanding and patience throughout this project.

- The staff of the Department of Geology, Geography and Environmental Studies, for their continued support and guidance.

- Dr Pieter de Necker, for his editorial work on this document, insightful suggestions and for keeping me motivated.

- Mr Bennie Schloms, who was always willing to provide advice and share his expertise.

- Prof Hannes van der Merwe, my promotor, for his continued motivation, support and timely suggestions. 


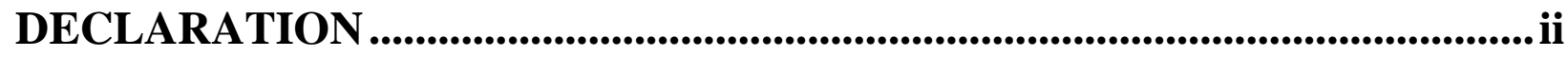

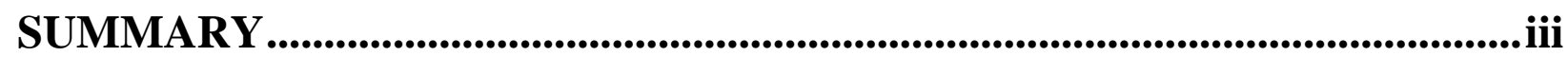

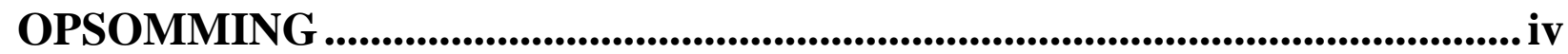

ACKNOWLEDGEMENTS............................................................................ vi

CONTENTS ................................................................................................................. vii

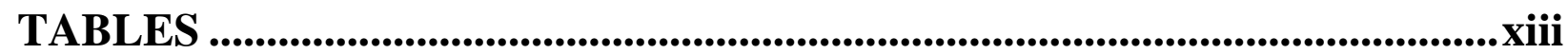

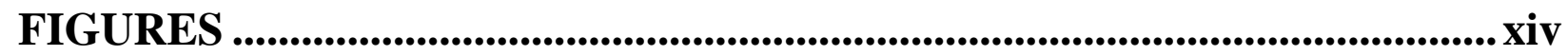

ACRONYMS AND ABBREVIATIONS .........................................................xvii

CHAPTER 1: TOWARDS IMPROVED SPATIAL DECISION MAKING.. 1

1.1 GEOGRAPHICAL INFORMATION SYSTEMS: AN OVERVIEW .........................2

1.2 SPATIAL DECISION SUPPORT SYSTEMS AND GIS............................................... 4

1.3 THE INTERNET AND GIS COMMUNICATION .......................................................

1.4 WEB MAPPING FOR SPATIAL DECISION SUPPORT …………………................. 7

1.5 RESEARCH PROBLEM FORMULATION ……..........................................................

1.6 RESEARCH AIM AND OBJECTIVES ...................................................................9

1.7 THE STUDY REGION .................................................................................................... 10

1.8 RESEARCH METHODOLOGY AND AGENDA …..................................................11

CHAPTER 2: THE PRINCIPLES AND PRACTICE OF LAND

SUITABILITY ANALYSIS ................................................. 14

2.1 LAND EVALUATION APPROACHES.....................................................................14

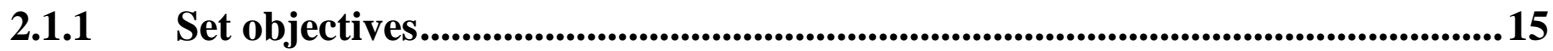

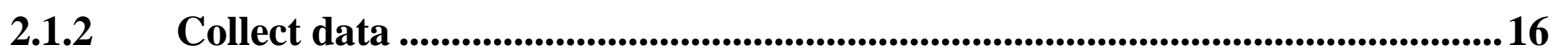

2.1.3 Identify land uses............................................................................................16

2.1.4 Specify land use requirements .............................................................................16

2.1.5 Map land units........................................................................................................17

2.1.6 Determine land properties spatially ......................................................................18

2.1.7 Analyse land units for suitability ........................................................................ 18

2.1.8 Present results...................................................................................................... 18

2.2 BOOLEAN OVERLAY ....................................................................................... 19

2.3 MULTI-CRITERIA DECISION MAKING ............................................................21

2.3.1 The MCDM procedure ..............................................................................................22 


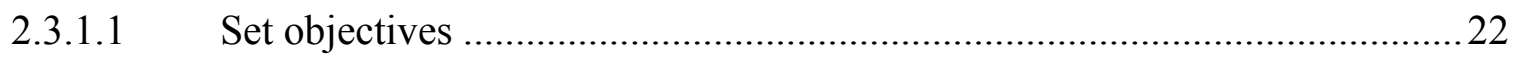

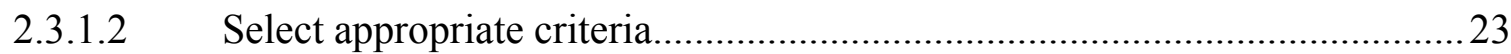

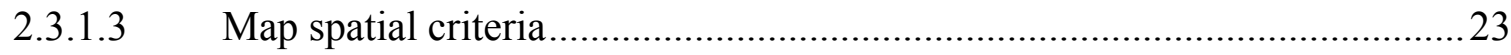

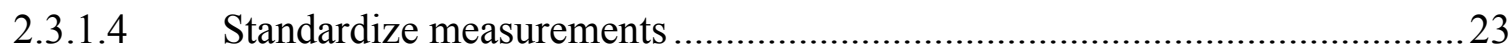

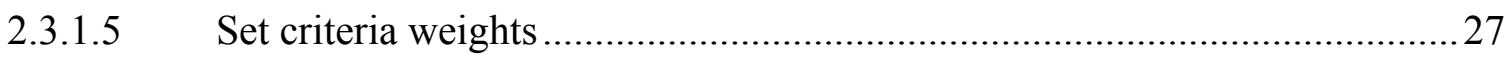

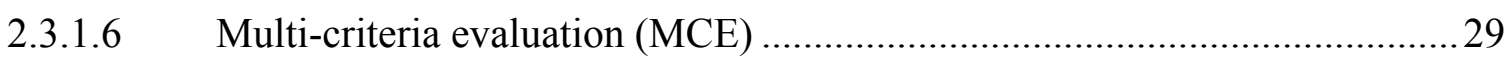

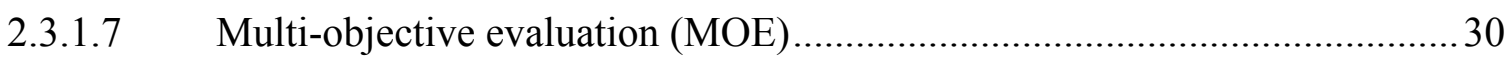

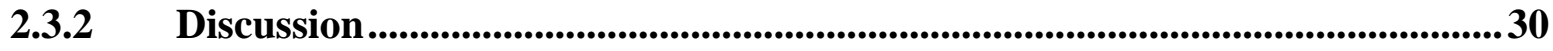

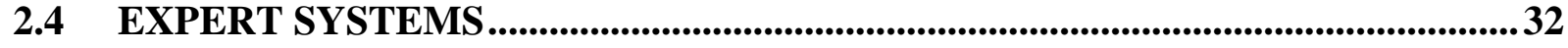

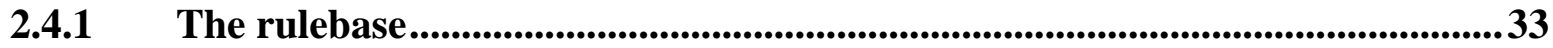

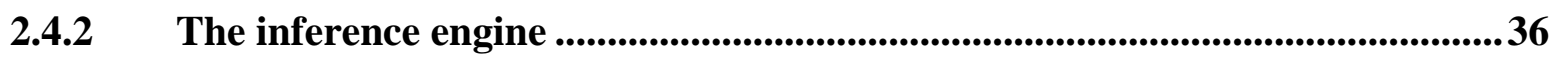

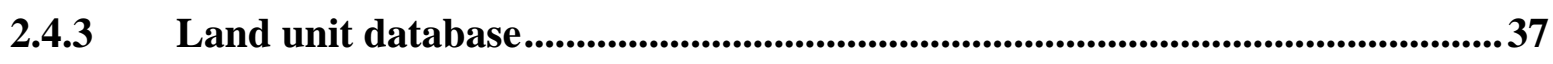

2.4.4 Existing land evaluation systems ................................................................37

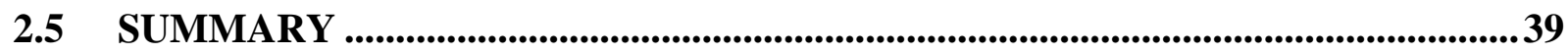

CHAPTER 3： WEB MAPPING TECHNOLOGY ...................................... 40

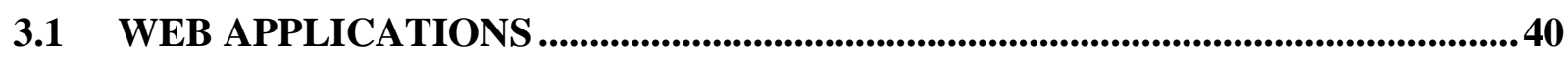

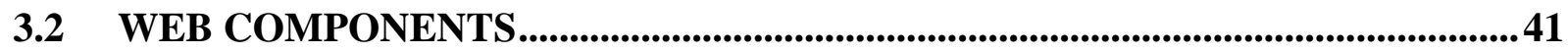

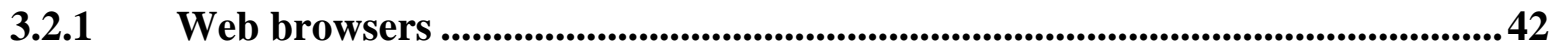

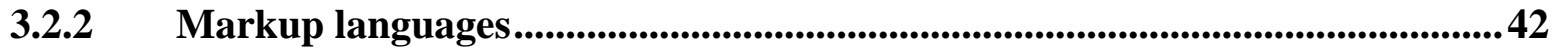

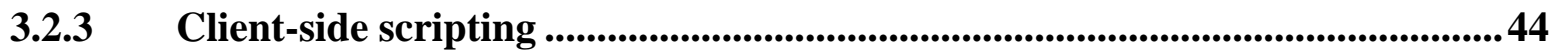

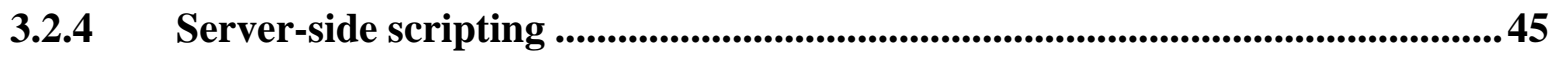

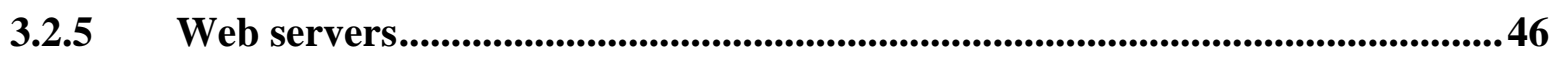

3.2.6 Relational database management systems .................................................47

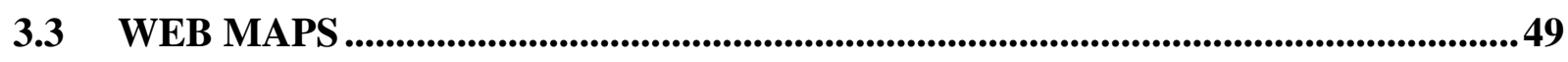

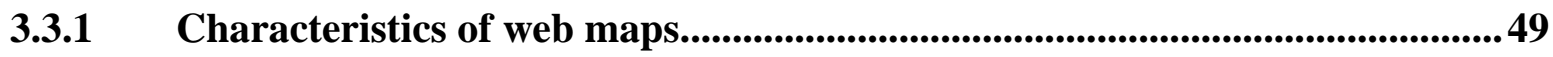

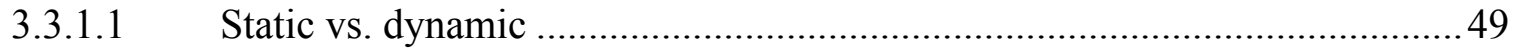

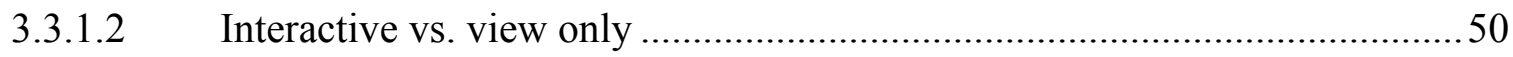

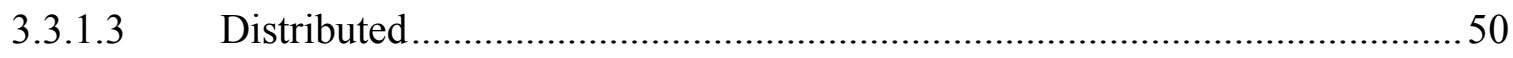

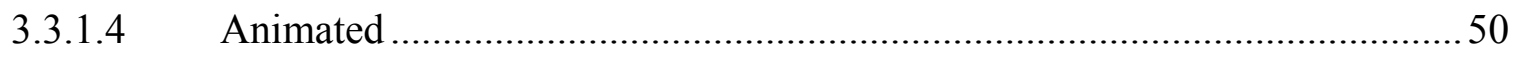

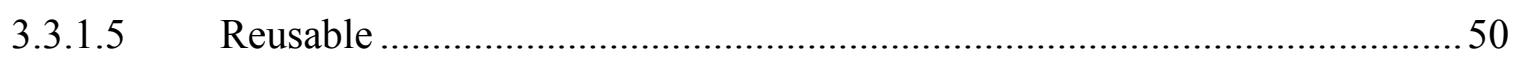

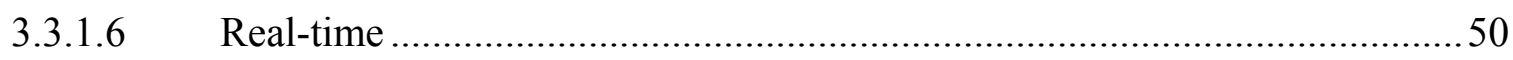

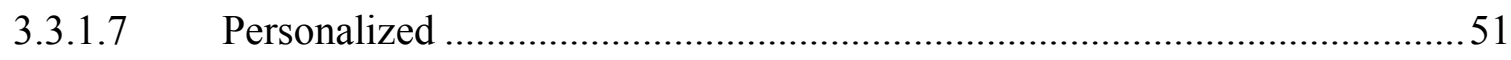

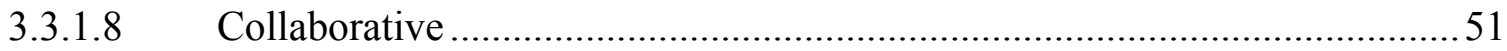

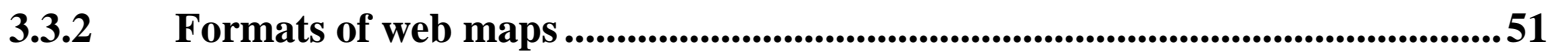

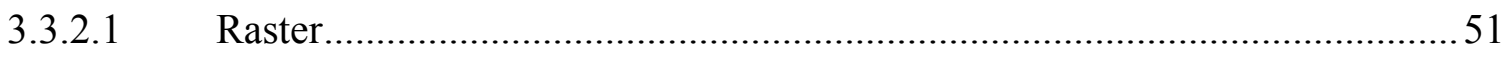




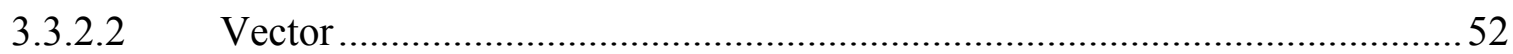

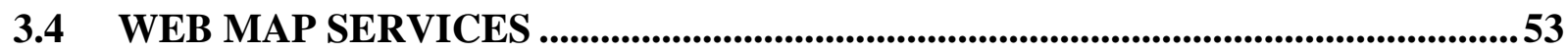

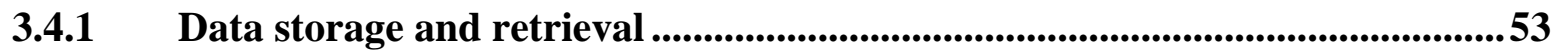

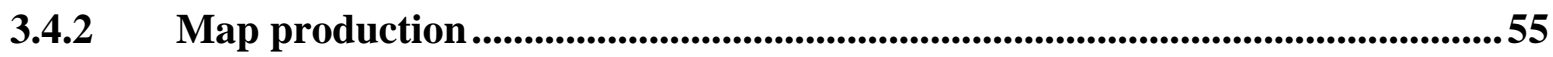

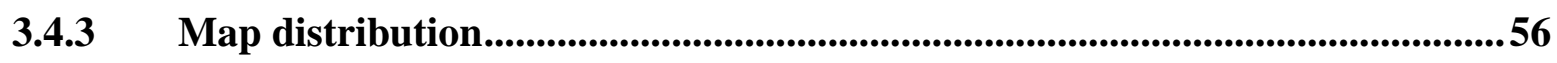

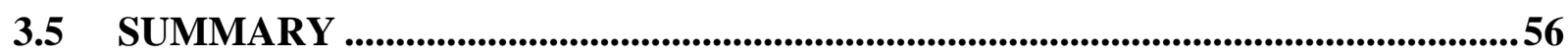

CHAPTER 4: REQUIREMENT ANALYSIS AND DESIGN OF CLUES .. 58

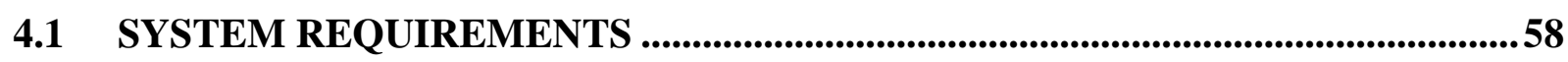

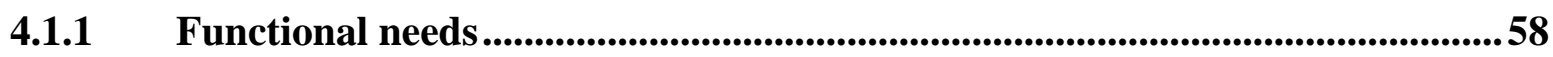

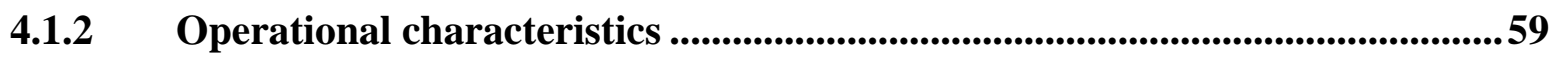

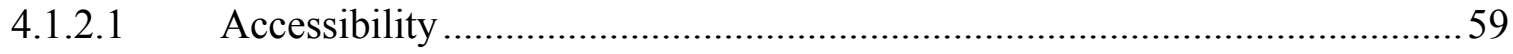

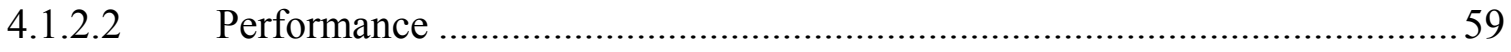

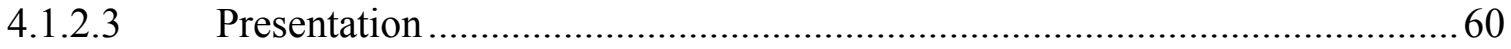

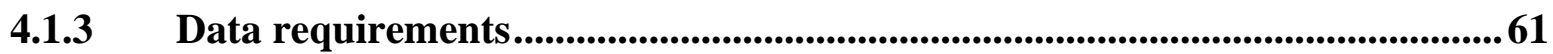

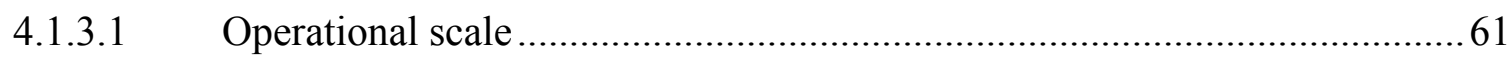

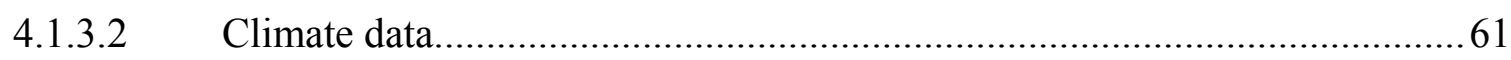

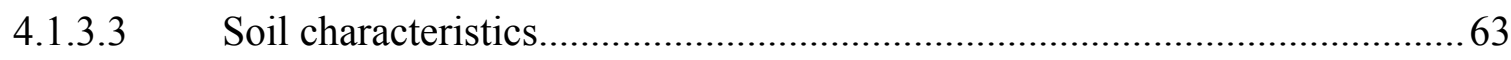

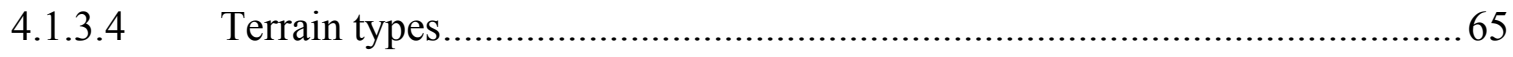

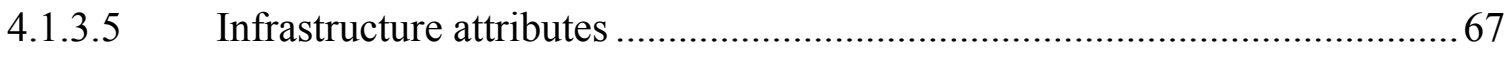

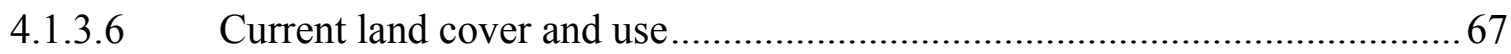

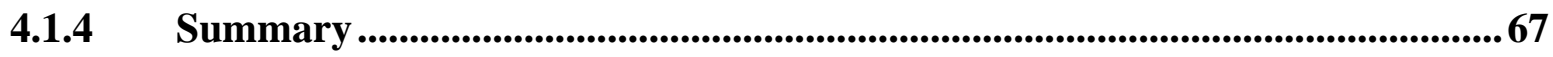

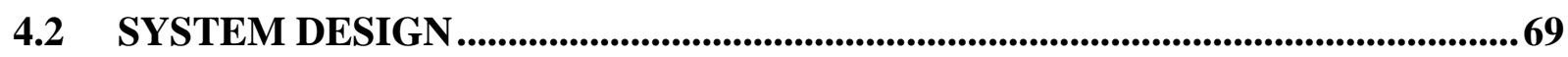

CHAPTER 5: LAND PROPERTY DATA COLLECTION ....................... 72

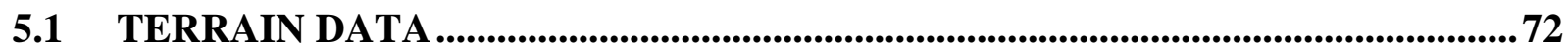

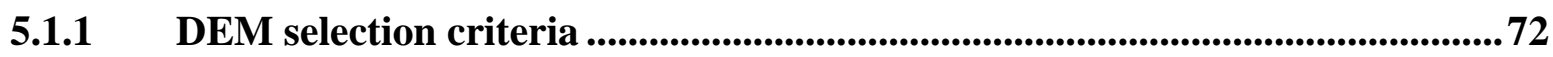

5.1.2 Existing Western Cape DEM .................................................................74

5.1.3 DEM accuracy assessment.....................................................................75

5.2 WESTERN CAPE SOIL INFORMATION ................................................................77

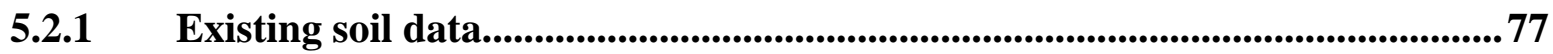

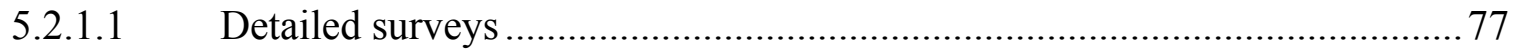

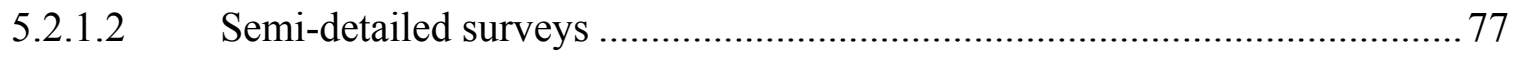

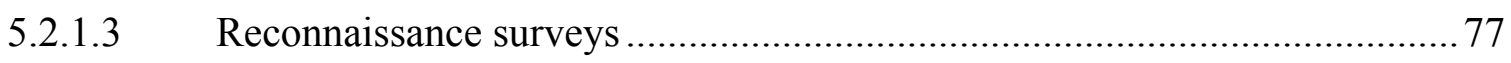

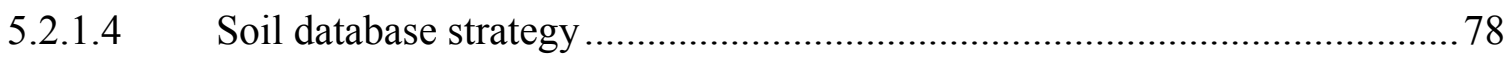

5.2.2 Land types information system................................................................................. 78

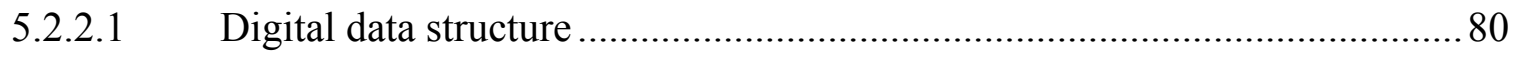




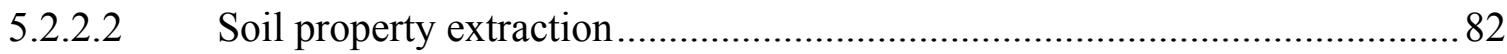

5.3 WESTERN CAPE CLIMATE INFORMATION ..................................................87

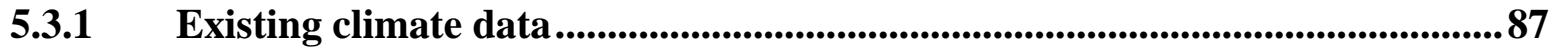

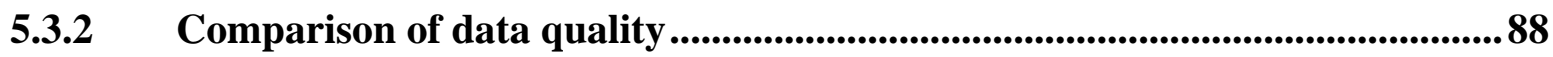

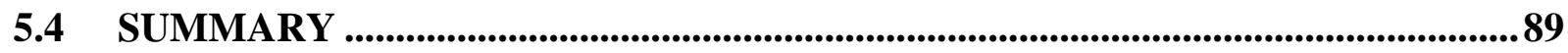

CHAPTER 6: DEVELOPING THE LAND UNIT DATABASE....................91

6.1 LAND COMPONENT MAPPING TECHNIQUES .............................................91

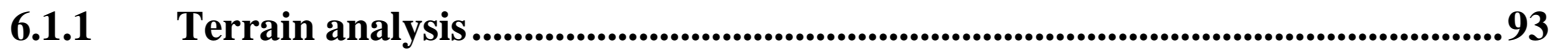

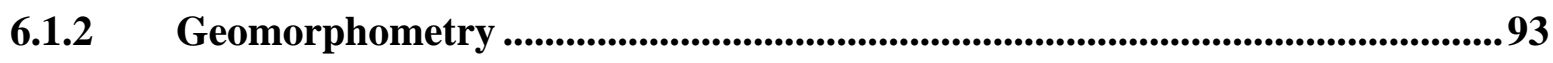

6.1.3 Automated mapping....................................................................................................994

6.2 Automated component mapping with ALCoM ................................................................95

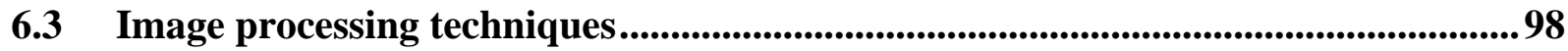

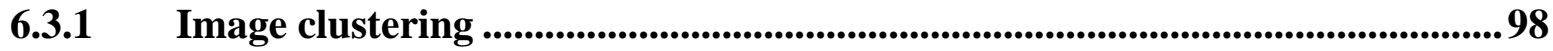

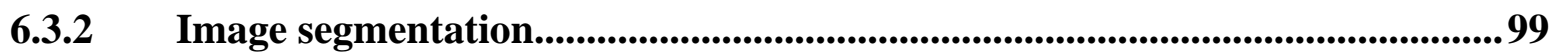

6.4 Comparison of ALCoM and MRS.................................................................... 103

6.5 SEGMENTING THE WESTERN CAPE .............................................................. 103

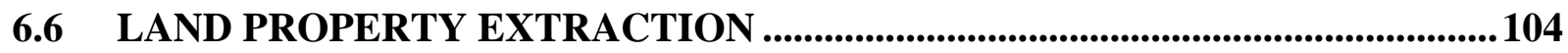

6.7 STORAGE ................................................................................................................... 105

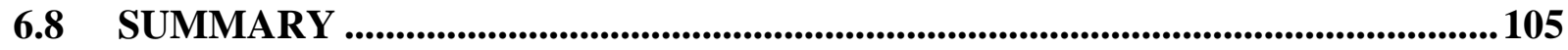

CHAPTER 7: DEVELOPING THE KNOWLEDGE BASE ........................ 107

7.1 LOGICAL DATA MODELLING METHODOLOGY .......................................... 107

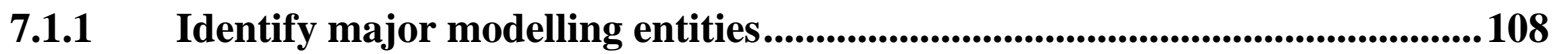

7.1.2 Determine operational relationships between entities ......................................108

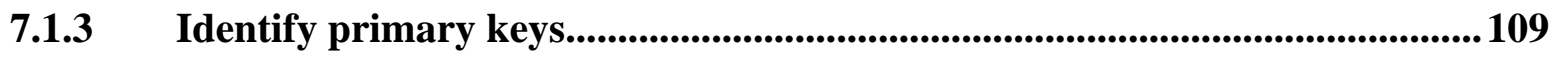

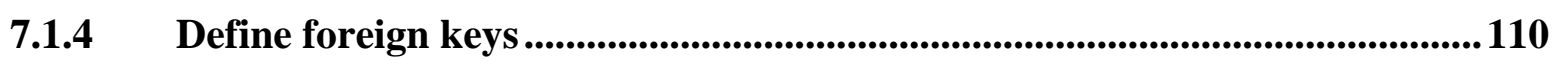

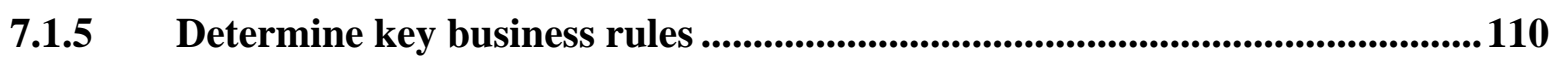

7.1.6 Add remaining non-key attributes.........................................................................110

7.1.7 Normalize data structure ................................................................................................. 112

7.1.8 Specify additional attribute business rules ....................................................113

7.1.9 Combine user views.......................................................................................114

7.1.10 Integrate with existing data models...................................................................... 114

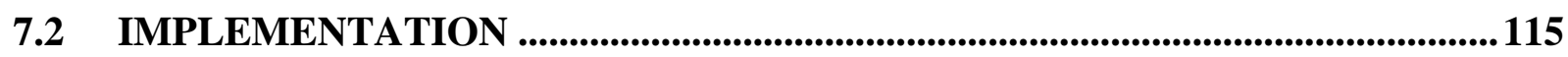

CHAPTER 8: DEVELOPMENT OF THE CLUES WEBSITE .................. 117

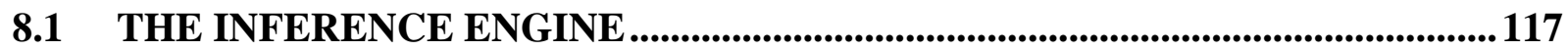

8.1.1 Suitability calculation procedure ...............................................................117 
8.1.2 Membership value calculation ...................................................................... 118

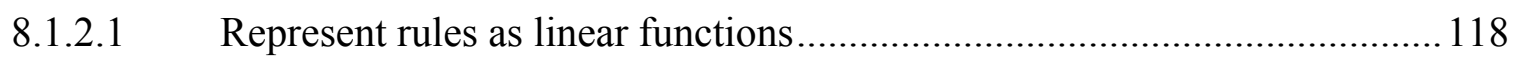

8.1.2.2 Determine membership function equations.............................................. 119

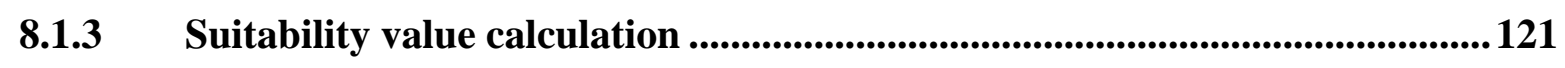

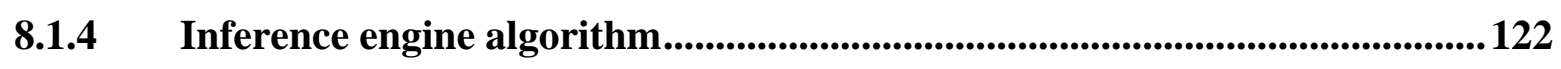

8.2 THE WEB MAP SERVICE AND WEB SERVER............................................... 124

8.2.1 Choice of software and hardware ..................................................................... 124

8.2.2 WMS configuration...................................................................................... 125

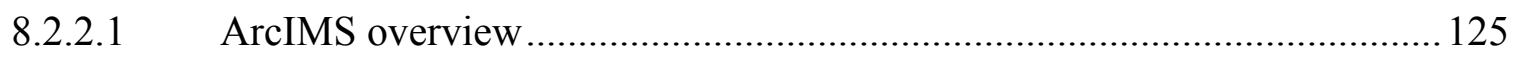

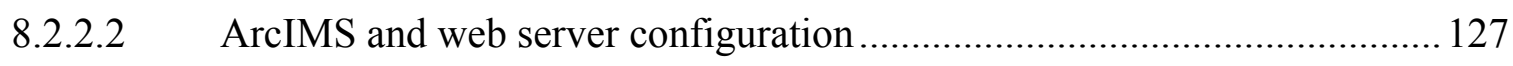

8.3 Development of a user-friendly GUI ........................................................................... 128

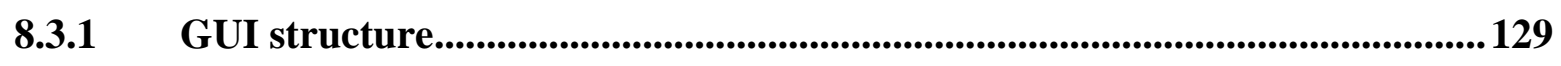

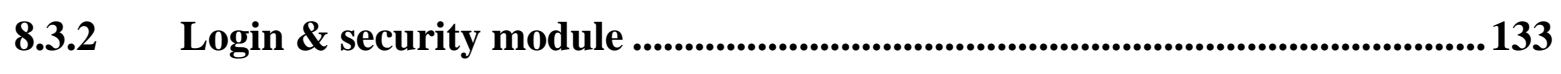

8.3.3 Menu module .................................................................................... 135

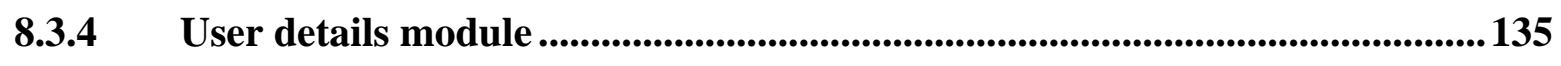

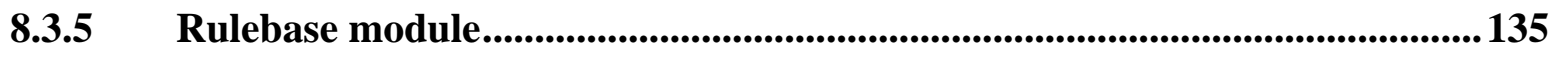

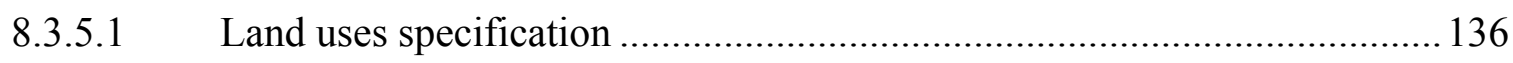

8.3.5.2 Land use requirements specification ................................................... 137

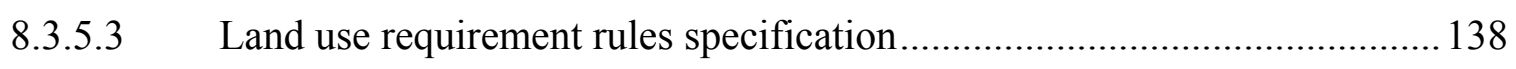

8.3.6 Projects module ....................................................................................... 139

8.3.7 Analyse \& map module............................................................................... 140

CHAPTER 9: DEMONSTRATIONS OF CLUES ................................... 143

9.1 SETTING RULES FOR PERENNIAL CROPS ............................................... 144

9.1.1 Terrain requirement rules......................................................................... 144

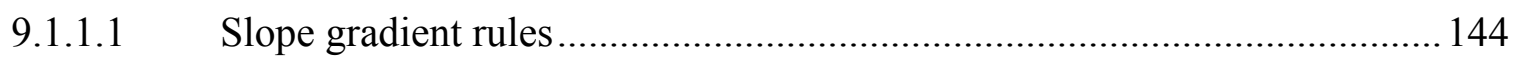

9.1.1.2 Aspect, curvature and elevation ......................................................... 148

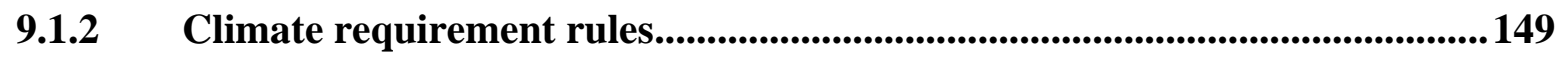

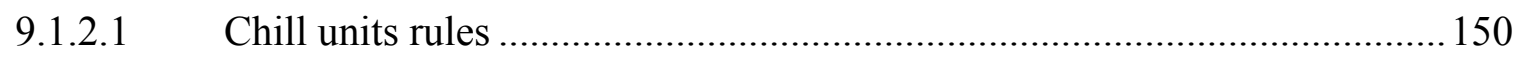

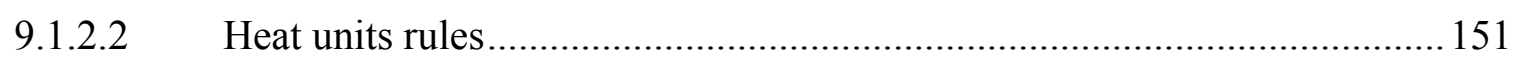

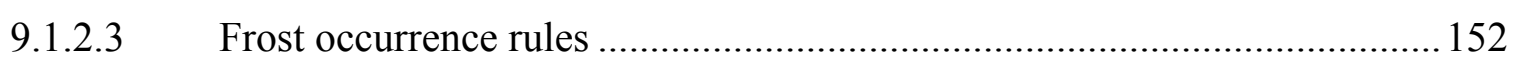

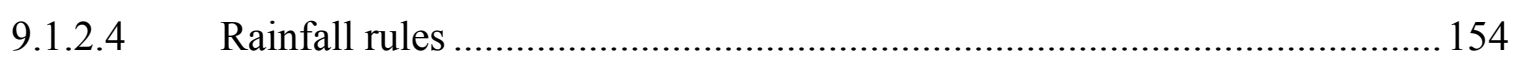

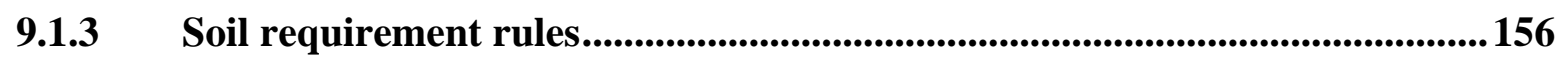

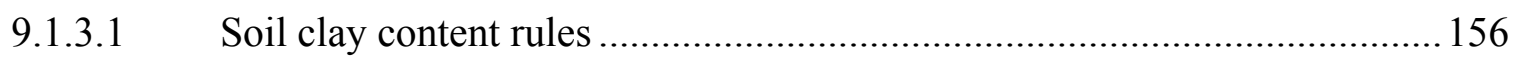

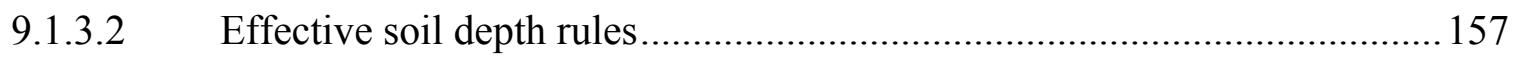

9.1.4 Current land uses and wetlands requirement rules .....................................159 


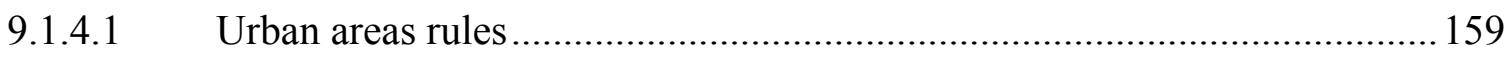

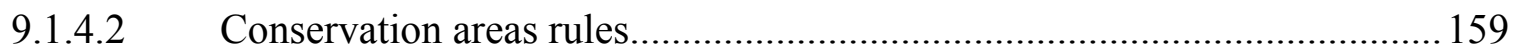

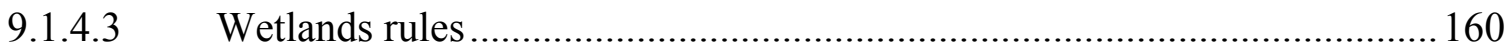

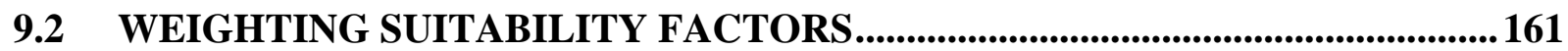

9.3 CASE STUDY SUITABILITY ANALYSIS AT VARYING SCALES.................163

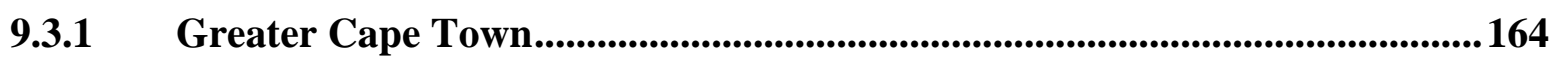

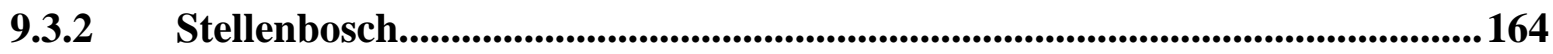

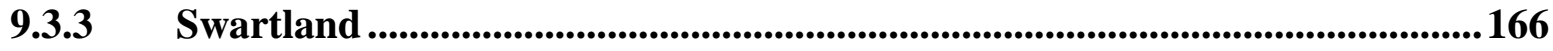

9.3.4 Rural Malmesbury …............................................................................................... 166

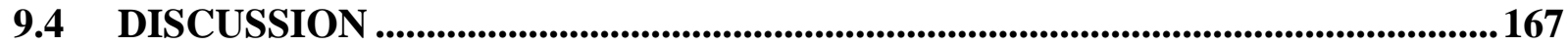

CHAPTER 10: EVALUATION OF THE RESEARCH................................. 169

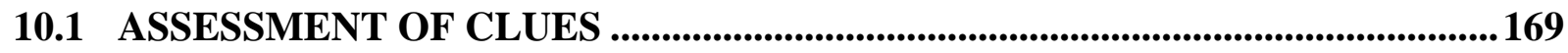

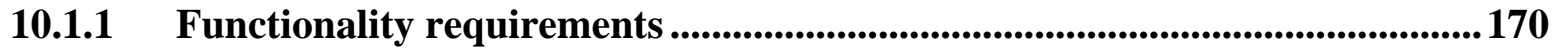

10.1.2 Operational performance requirements ................................................172

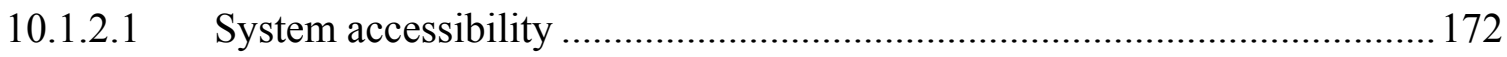

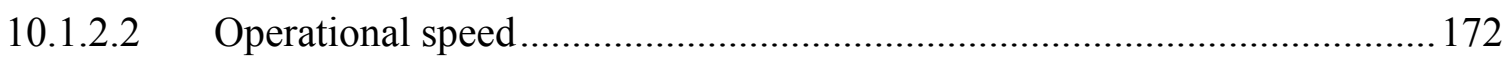

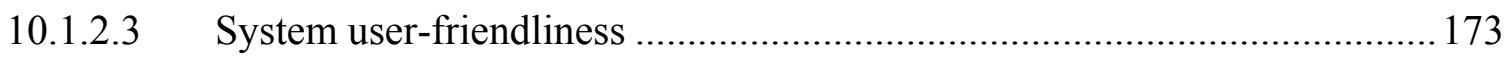

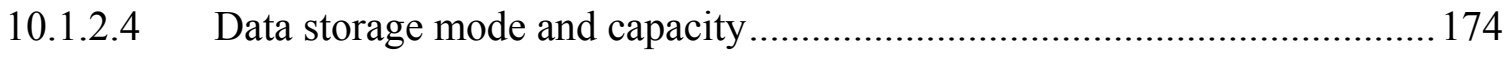

10.1.3 Data requirements................................................................................................. 174

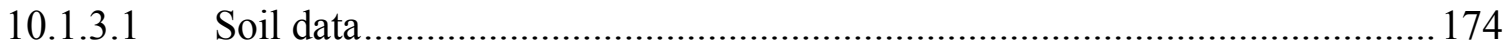

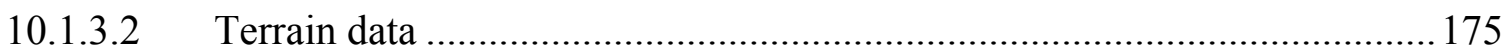

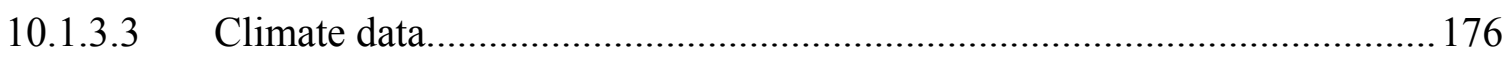

10.1.4 Scale, scalability and flexibility requirements ................................................ 176

10.2 POTENTIAL OF WEB TECHNOLOGY FOR SDSS DEVELOPMENT............178

10.3 RESEARCH OBJECTIVES REVISITED ...........................................................180

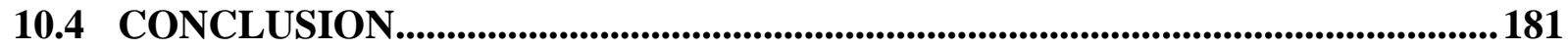

REFERENCES ............................................................................................. 183

PERSONAL COMMUNICATIONS.................................................................... 201

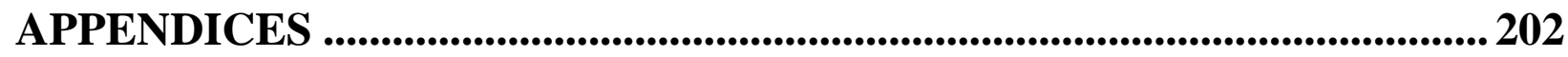




\section{TABLES}

Table 2-1 Scale of analytical hierarchy process (AHP) comparisons .................................... 28

Table 2-2 AHP comparison matrix for perennial crops ..........................................................28

Table 2-3 Example of five Boolean rules specifying effective soil depth requirements for

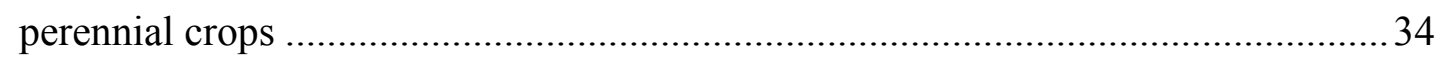

Table 2-4 Example of two Boolean and five fuzzy rules specifying effective soil depth

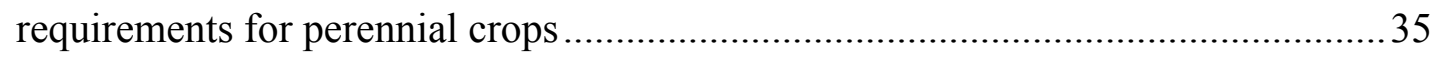

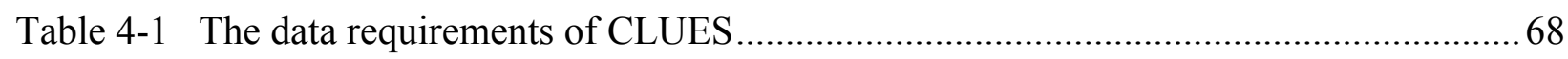

Table 5-1 Vertical error in the WCDEM and the STRM DEM .............................................. 76

Table 5-2 Accuracy summary of existing climatic data for the Western Cape.......................... 88

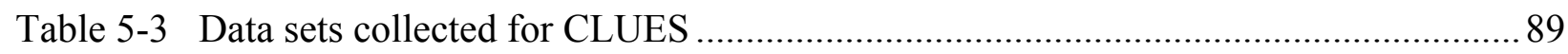

Table 6-1 Statistical comparison of land components mapped using ALCoM and MRS ........ 101

Table 7-1 Relationship matrix of entities in the knowledge base ........................................ 109

Table 7-2 Entity attributes in the knowledge base ................................................................ 111

Table 7-3 Business rules for USER entity in the knowledge base ........................................ 113

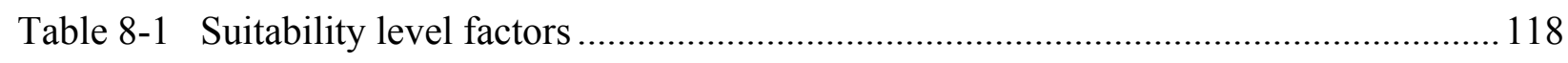

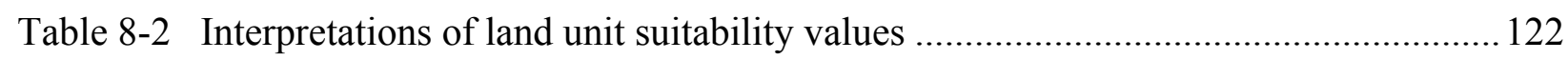

Table 8-3 Tools available for manipulating the map frame ................................................ 141

Table 9-1 Calculated suitability values and levels for selected slope values.......................... 147

Table 9-2 AHP comparison matrix of weights assigned to land use requirements.................. 162 


\section{FIGURES}

Figure 1-1 The Western Cape province, South Africa. 11

Figure 1-2 Research design for developing CLUES, a web-based SDSS for the Western Cape 12

Figure 2-1 The steps in a land evaluation process 15

Figure 2-2 Intersect and union Boolean overlay operations 20

Figure 2-3 A step-wise procedure for multi-criteria decision making..... 22

Figure 2-4 Linear scaling of effective soil depth for perennial crop suitability... 24

Figure 2-5 Ascending (a) and descending (b) S-membership functions 26

Figure 2-6 Effective soil depth (a) and soil acidity (b) membership functions for perennial crops

Figure 2-7 Boolean constraint of effective soil depth for perennial crops. 27

Figure 2-8 Graduated shades used to visualize suitability levels of factors and results ..... 30

Figure 2-9 Procedure for an expert system land suitability analysis. 33

Figure 2-10 Levels of suitability of effective soil depths for perennial crops using Boolean classification

Figure 2-11 Levels of suitability of effective soil depths for perennial crops using fuzzy classification

Figure 3-1 Example of a HTML document.

Figure 3-2 Example of an email stored as XML.

Figure 3-3 Example of JavaScript code that displays an error message when the web page is opened.

Figure 3-4 A SQL statement using the SELECT and JOIN operators..... 48

Figure 3-5 Classification of web maps

Figure 4-1 Soil texture triangle showing the twelve major textural classes and particle size scales .....

Figure 4-2 The components of CLUES... 69

Figure 5-1 Selection of reference points used in the DEM accuracy assessment ..................... 75

Figure 5-2 Land type Ca6 in memoir format ........................................................................ 79

Figure 5-3 Land type polygon (a) and its associated attribute information (b)........................ 80

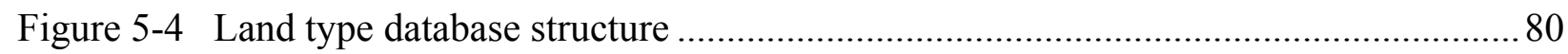

Figure 5-5 Conceptual view of land type object levels ........................................................ 82

Figure 5-6 Effective soil depth derived from land type data................................................... 84

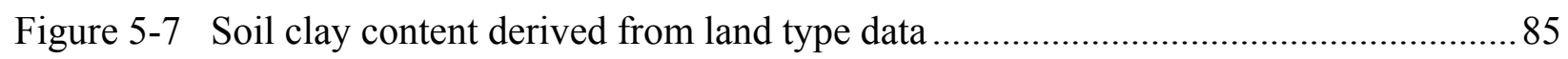

Figure 5-8 Soil mechanical limitations derived from the land type data ................................. 86 
Figure 6-1 Two hypothetical hillslopes, each consisting of a sequence of five land components

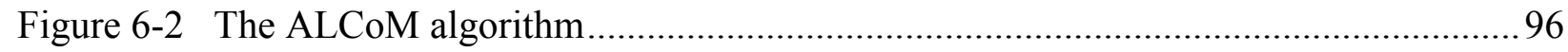

Figure 6-3 Location of the test area for ALCoM .................................................................. 97

Figure 6-4 Detailed view of the test area, with selected terrain features indicated.................... 97

Figure 6-5 Land component boundaries mapped by ALCoM ................................................. 97

Figure 6-6 A conceptual comparison of clusters (a) and segments (b) in relation to attributes A

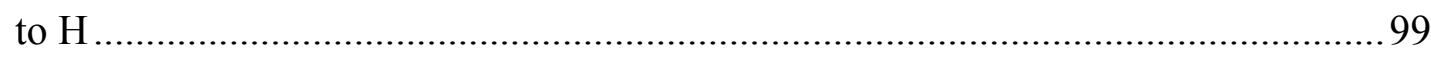

Figure 6-7 Land components mapped by multi-resolution segmentation................................ 102

Figure 7-1 Logical data model diagram of CLUES knowledge base .................................... 115

Figure 8-1 Levels of suitability of effective soil depths for perennial crops using linear fuzzy classification

Figure 8-2 Symmetrical (a) and asymmetrical (b) fuzzy rules deconstructed to two lines, A and

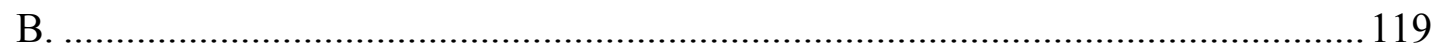

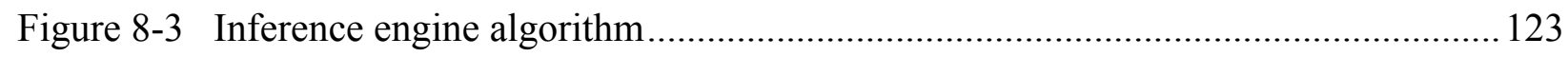

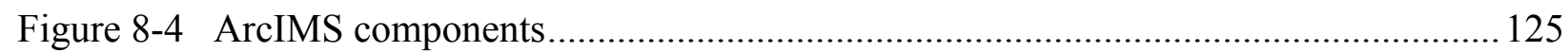

Figure 8-5 Main steps followed to produce a suitability map using CLUES ......................... 129

Figure 8-6 GUI pages and linkages in CLUES ................................................................... 131

Figure 8-7 Code of the main page showing interaction between HTML, JavaScript and Visual

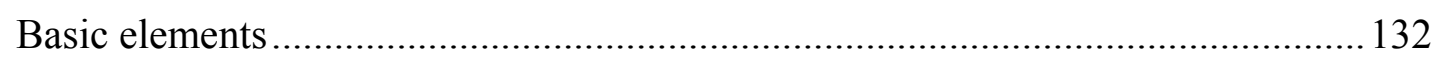

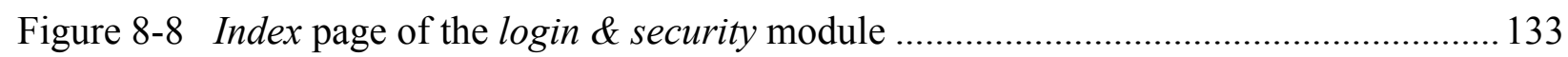

Figure 8-9 Register page of the login \& security module .................................................... 134

Figure 8-10 Register_success page of the login \& security module ....................................... 134

Figure 8-11 Main and banner menus as shown on the main page ........................................ 135

Figure 8-12 A list of land uses owned by the current user shown on the landuses page.......... 136

Figure 8-13 Requirements for land use $\mathrm{A}$ as listed on the req page ..................................... 137

Figure 8-14 Requirement rules for a hypothetical land use as displayed on the rules page ..... 138

Figure 8-15 Information about a user's projects shown on the projects page ......................... 139

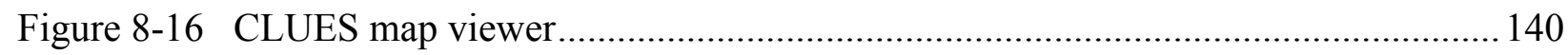

Figure 9-1 The landuse_create page used to create a new land use called 'perennial crops' .. 144

Figure 9-2 Slope gradient (\%) added as a requirement for perennial crops ........................... 145

Figure 9-3 Rules defining suitability levels for slope gradient ......................................... 146

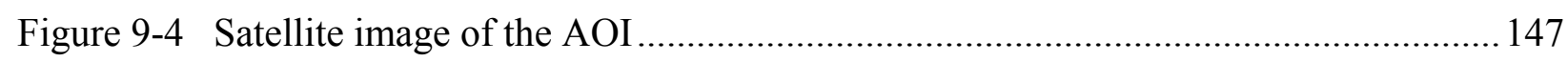

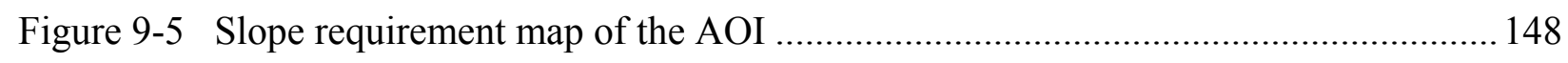

Figure 9-6 Suitability results overlaying a satellite image for orientation purposes................ 149 


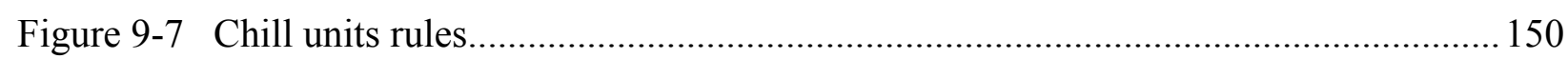

Figure 9-8 Chill units requirement map of the AOI ............................................................ 151

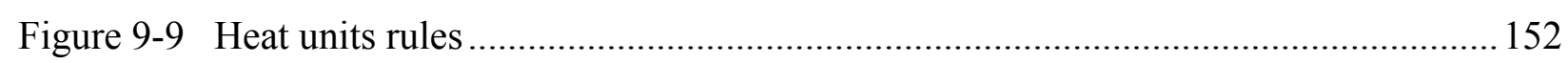

Figure 9-10 Heat units requirement map of the AOI .......................................................... 153

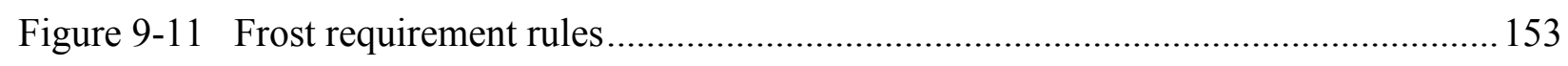

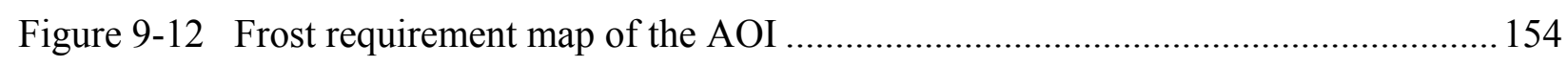

Figure 9-13 Mean annual rainfall requirement rules.............................................................. 155

Figure 9-14 Mean annual rainfall requirement map of the AOI ......................................... 155

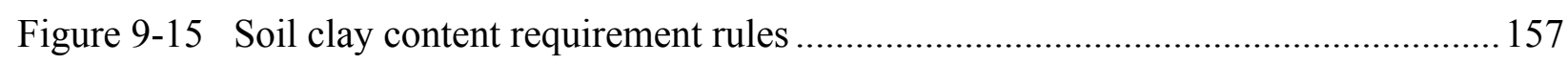

Figure 9-16 Soil clay content suitability map of the AOI ................................................... 157

Figure 9-17 Effective soil depth requirement rules............................................................. 158

Figure 9-18 Effective soil depth requirement map of the AOI ............................................ 159

Figure 9-19 Urban areas in the AOI considered permanently unsuitable ............................... 160

Figure 9-20 Conservation areas in the AOI considered permanently unsuitable..................... 161

Figure 9-21 Wetlands in the AOI considered permanently unsuitable ................................. 161

Figure 9-22 List of perennial crop requirements with weights shown on the req page ............ 163

Figure 9-23 Suitability map for perennial crops in the greater Cape Town AOI..................... 164

Figure 9-24 A compilation of CLUES screen captures showing detailed maps of (a) the suitability analysis result, (b) the suitability overlay, (c) land unit outlines, and (d) the satellite image of the area north of Stellenbosch............................................. 165

Figure 9-25 A compilation of CLUES screen captures showing (a) the satellite image and (b) the suitability analysis results for the Swartland area .......................................... 166

Figure 9-26 A compilation of CLUES screen captures showing detailed maps of (a) the satellite image and (b) the suitability analysis results for the area west of Malmesbury ..... 167 


\section{ACRONYMS AND ABBREVIATIONS}

ADF

ADO

AEZWIN

AHP

AHPP

ALCoM

ALES

AOI

API

ARPANET

ASP

$\mathrm{BDE}$

CAD

CAPE

CDSM

CEMS

CFML

CFR

CGA

CGI

CGIS

CLUES

$\mathrm{COM}$

CPU

CR

CSIR

$\mathrm{CU}$

DBMS

DEM

DHTML

DIME

DSS
Application development framework

ActiveX data objects

Agro-Ecological Zone for Windows

Analytical hierarchy process

AHP Program

Automated Land Component Mapper

Automated Land Evaluation System

Area of interest

Application programming interface

Advanced Research Projects Agency Network

Active server pages

Borland database engine

Computer-aided design

Cape Action for People and the Environment

Chief Directorate Surveys and Mapping

Cape Environmental Management System

ColdFusion markup language

Cape floristic region

Centre for Geographical Analysis

Common gateway interface

Canada Geographic Information System

Cape Land Use Expert System

Component object model

Central processing unit

Consistency ratio

Council for Scientific and Industrial Research

Chill units

Database management system

Digital elevation model

Dynamic hypertext markup language

Dual independent map encoding

Decision support systems 
EROS

ESRI

FAO

FMF

GDD

GIF

GIS

GPS

GRASS

GUI

HTML

HTTP

HU

IBM

IIS

IP

ISCW

ISLE

ISODATA

JPEG

LAN

LBS

LDM

LDMD

LEIGIS

LIDAR

LT

MADM

MAE

MCA

MCDM

MCE

MicroLEIS

MODM

MOE
Earth Resources Observation and Science

Environmental Systems Research Institute

Food and Agriculture Organization

Fuzzy membership function

Growing degree-days

Graphics interchange format

Geographical information system

Global positioning system

Geographic resources analysis support system

Graphical user interface

Hypertext markup language

Hypertext transfer protocol

Heat units

International Business Machines

Internet Information Services

Internet protocol

Institute of Soil, Climate and Water

Intelligent System for Land Evaluation

Iterative self-organizing data analysis technique algorithm

Joint photographic experts group

Local area network

Location based services

Logical data modelling

Logical data model diagram

Land Evaluation using an Intelligent Geographical Information System

Light detecting and ranging

Land type

Multi-attribute decision making

Mean absolute error

Multi-criteria analysis

Multi-criteria decision making

Multi-criteria evaluation

Mediterranean Land Evaluation Information System

Multi-objective decision making

Multi-objective evaluation 
MRS

MS

MS-DOS

NASA

NDA

ODBC

OGC

OS

OWA

PDF

PHP

PNG

RADAR

RAM

RDBMS

RGB

RLE

RMSE

SAAAC

SANBI

SAWS

SAWIS

SDE

SDSS

SGML

SGV

SQL

SRTM

SVG

SWF

TIFF

TOC

TU

TWI

UNEP
Multi-resolution segmentation

Microsoft

Microsoft Disk Operating System

National Aeronautics and Space Administration

National Department of Agriculture

Open database connection

Open Geospatial Consortium

Open source

Ordered weighted averaging

Portable document format

PHP hypertext preprocessor

Portable network graphics

Radio detecting and ranging

Random access memory

Relational database management system

Red, green and blue

Run length encoding

Root mean square error

South African atlas of agrohydrology and -climatology

South African National Biodiversity Institute

South African Weather Services

South African Wine Information \& Systems

Spatial data engine

Spatial decision support systems

Standard generalized markup language

Slope gradient variance

Structured query language

Shuttle Radar Topography Mission

Scalable vector graphics

Shock wave flash

Tagged image file format

Table of contents

Terrain units

Topographical wetness index

United Nations Environment Programme 
URL Uniform resource locator

USGS

United States Geological Survey

UTM

Universal transverse Mercator

WCCG

Western Cape climate grids

WCDEM

Western Cape Digital Elevation Model (20m resolution)

WCDEM80

Western Cape Digital Elevation Model (80m resolution)

WLC

Weighted linear combination

WMS

Web map service

WWW

World Wide Web

XHTML

Extensible hypertext markup language

XML

Extensible markup language 


\section{CHAPTER 1: TOWARDS IMPROVED SPATIAL DECISION MAKING}

"GIS is an unfinished revolution...Now, more than ever, geographic awareness, thinking and curiosity remain the key to getting the best from a tool that can help support Earth-changing decision making." (Van Wyngaarden \& Waters 2007: s.p.).

During their forty years of existence, geographical information systems (GIS) have revolutionized geographical analysis and spatial decision support and greatly enhanced our understanding of the real world through their mapping and spatial modelling capabilities. Although the GIS industry is continuing to grow as the software becomes more powerful, less expensive and more user-friendly GIS remain the domain of a select few who can operate and afford these systems.

Since the introduction of web mapping tools such as Yahoo Maps, Microsoft Virtual Earth and NASA World Wind, accessibility to geographical information has escalated. Web mapping software enables anyone with access to a computer and the Internet to explore geographical data online and produce maps on demand. An increasingly popular web mapping tool is Google Earth which bundles seamless satellite imagery and other geographical information into a tool that is simple and easy to use. Unlike GIS that require users to know how data is acquired, prepared, stored and queried, Google Earth allows users to use the geotechnology in an intuitive, interactive manner.

Web mapping products such as Google Earth have a very narrow range of functionality. In contrast to GIS that focus on spatial data capture, storage, manipulation, analysis and presentation, the function of web mapping tools is to visualize and communicate geographical data in a highly user-friendly manner. The positive impact of web mapping tools on geographical awareness, as well as the popularity of these systems, suggest that GIS have not yet developed to a level where all professionals can use the technology to support spatial decisions and enhance productivity. GIS functionality is not yet accessible enough to significantly enhance the geographical awareness of the general public. According to Van Wyngaarden \& Waters (2007) GIS should do more to encourage geographical thinking and to demonstrate the principles of geographical science. The functional gap between web mapping tools and GIS should be closed to make GIS functionality more accessible to all professionals and the public in general. The adoption of these capabilities by a wider audience will promote geographical awareness and ultimately lead to better spatial decisions.

This chapter sets out to provide critical perspective concerning GIS and its development as spatial decision support system, lately also via the Internet and web mapping. It concludes with 
formal statements of the research problem, aim and objectives, describes the study region and introduces the research methodology applied in the dissertation.

\subsection{GEOGRAPHICAL INFORMATION SYSTEMS: AN OVERVIEW}

The value of computer technology for spatial applications was first recognized in the early 1960s when Canada's Department of Forestry and Rural Development set out to map and compile an inventory of its natural resources in order to manage land more effectively. Realizing that the manual production of the maps required considerable time and funds, the project team decided to use computer technology. In conjunction with IBM, the first GIS - called the Canada Geographic Information System (CGIS) - was developed and completed in 1963 (DeMers 2005).

In 1967 the United States government needed a similar tool to support the automatic referencing and aggregation of their census records. The dual independent map encoding (DIME) program was developed and, although the applications for which CGIS and DIME were developed were quite different, the many similarities were quickly recognized. As a result, the Harvard Laboratory for Computer Graphics and Spatial Analysis, under direction of Howard Fisher, developed the first general purpose GIS, called ODYSSEY GIS, which was completed in 1977 (Longley et al. 2002). Other GIS followed.

The computer systems on which early GIS operated were prohibitively expensive and only a select few governmental and educational institutions could afford ownership. The dramatic fall in prices of computer hardware in the 1980s changed this. Smaller institutions started to implement GIS and natural resources agencies particularly, such as the Environmental Systems Research Institute (ESRI), drove the development of better software. During the 1990s hardware and software continued to be improved, leading to further price reductions and an explosion in the number of users and applications (Longley et al. 2002). Today geospatial technology, along with nanotechnology and biotechnology, is one of the three most important emerging industries in the United States (U.S. Department of Labor 2006).

Because of the diverse applications of GIS there is no generally accepted definition for the technology. Opinions about what is essential for a system to be called a GIS differ according to each user's needs. Most users agree, however, that GIS are special computer systems that capture, store, query, analyse and display geographically referenced data (Chang 2006; Clarke 2003; DeMers 2005). But the property which truly differentiates GIS from other spatial systems, such as Computer-aided design (CAD), is their ability to analyse spatial data (Clarke 2003). Spatial analysis is defined by Longley et al. (2002: 278) as "the process by which we turn raw 
spatial data into useful information...[to]...add value, support decisions, and reveal patterns and anomalies that are not immediately obvious."

In order to conduct spatial analysis, a spatial database is required. Geographical data is expensive to collect and capture and can be the most expensive component of GIS. Fortunately GIS are maturing and more data is becoming available and many governments, including that of South Africa, have made state-owned data freely accessible to the public (South Africa 2000). In spite of the improved policies and advances in technologies to aid data capture, such as remote sensing and global positioning systems (GPS), the establishment of spatial databases still impedes many GIS implementations.

For a GIS to operate, a computer system is required. The early GIS ran on bulky mainframes, which were later replaced with UNIX workstations and since the mid-1990s workstations have made way for personal (micro) computers running Microsoft (MS) Windows. Today nearly three quarters of GIS professionals use MS Windows, while only one quarter use UNIX-based systems (GISjobs.com 2006). This trend is continuing, primarily because of the affordability and computing power of modern personal computers.

Besides hardware, a GIS also requires specialized software to process spatial data. GIS software became considerably more affordable during the 1990s, mainly due to greater demand and technological advances. Even some free GIS software packages, such as GRASS (GRASS 2006) and LandSerf (Wood 2006), have become available. In spite of the availability of free GIS software, most GIS professionals use proprietary GIS software products. More than three quarters of GIS professionals currently use either ArcView or ArcGIS developed by ESRI, while Autodesk, Intergraph and other GIS software developers compete for the remaining market share (GISjobs.com 2006). The drop in prices, together with advances in providing user-friendly interfaces, are perceived to be the major reasons why GIS software sales more than doubled from 1995 to 2000 (Longley et al. 2002).

Undoubtedly the most important and least valued component of GIS are the people, also called 'brainware' (Chang 2006), who use and manage these systems. GIS are often regarded as a set of spatial tools (Clarke 2003) and it is the GIS operator's function to choose the correct set of tools for a specific application and to use these tools in the correct order and manner to achieve the desired results. A GIS is useless without human input.

The number of GIS users, estimated in 2000 to have been about three million worldwide, is rapidly increasing (Longley et al. 2002). GIS have become more accessible mainly owing to the lower costs of GIS software and hardware and the advent of intuitive, user-friendly desktop GIS. With these systems users can perform many spatial tasks with little GIS training, thus enabling 
many managers, scientists and decision makers to perform routine queries and analyses themselves instead of relying on dedicated GIS staff. This has opened a vast new market for GIS vendors, resulting in huge increases in software sales.

Increasing numbers of users, resulting from better access to GIS, is certainly good for the industry, but off-the-shelf desktop GIS cannot ensure that unskilled users choose the appropriate procedures ('tools') for a specific task or that they use appropriate procedures correctly. Such failings can lead to meaningless results or errors which might remain undetected by the casual user. One way to ensure that GIS users, skilled or unskilled, use the technology appropriately is to specialize a GIS, i.e. set it up so that it is used for a specific task only. The term 'specialized GIS' is somewhat ambiguous as GIS are by nature not specialized (i.e. can be used for many purposes), but it signifies that some operations are automated in such a way that there is little room for error. When GIS are customized to perform a combination of automated operations, they are often called spatial decisions support systems (SDSS).

\subsection{SPATIAL DECISION SUPPORT SYSTEMS AND GIS}

SDSS are decision support systems (DSS) with a spatial component. DSS were developed in the 1960s to aid decision making when problems are neither well structured nor unambiguous. These problems are referred to in the literature as ill-structured or semi-structured (Ascough et al. 2001; Densham 1991; Goodchild \& Densham 1990), i.e. they cannot be solved with an algorithm or a predefined sequence of operations. To solve such problems, predictive analysis is often required to present decision makers with different scenarios to explore the possible effects of their decisions. This type of interactive exploration enables a decision maker to develop a better understanding of a given problem. DSS are therefore not meant to provide solutions, but rather to support decisions.

DSS distinguish themselves from other information systems in that they require a database management system, analytical modelling capabilities, analysis procedures, and a user interface with display and report generators. DSS often use expert systems to interpret and analyse data. Expert systems are software programs that mathematically analyse subject-specific knowledge in the form of rules to solve problems.

In addition to the capabilities of DSS, SDSS should include a geographical database, mechanisms for spatial data input, representation of spatial relations and structures, spatial analysis functions and various output forms including maps (Ascough et al. 2001). SDSS can therefore be created by extending existing DSS to handle spatial data. 
When the sub-systems of a GIS (i.e. input, storage, management and analysis, and output) are compared to those of a SDSS, many similarities are evident, although a GIS cannot be regarded as a SDSS on its own as it lacks the interactivity and automation required for scenario generation. However, thanks to GIS's capability to handle spatial data, GIS and DSS are often combined to create SDSS (Agrell, Stam \& Fischer 2004). Such GIS/DSS implementations save development time as they make use of existing software.

Another approach to developing SDSS is to modify and customize GIS to incorporate the functionality of DSS (Basson 2005; Bester 2004; Mlisa 2007; Van Niekerk 1997; Varma, Ferguson \& Wild 2000). Most modern GIS allow developers to extend their functionality through programming. This approach has a cost benefit as no DSS licensing costs are applicable, although, as with the GIS/DSS approach, the user must still own a copy of the GIS software in order to use the system. In many cases users are managers or decision makers for whom the GIS software must be purchased especially to run the SDSS. This can be very costly and, because these SDSS (and users) rarely use the full GIS functionality, it is highly inefficient.

Some GIS developers offer component-based software development tools that allow spatial functionality to be incorporated in other non-GIS software. With these tool kits, GIS functions (components) can be seamlessly embedded in existing applications such as spreadsheets, digital atlases, and routing systems. A programmer can also create an entirely new SDSS (or even GIS) containing only the functionality that is needed for a specific application (Longley et al. 2002). This lowers the implementation cost per user as the developer purchases a tool kit once and pays a small licensing fee for each deployment (ESRI 2002c). However, the procedure requires additional development time and resources and is only viable when the number of users is large.

Because SDSS are designed for specific applications, many of the operations can be automated. This reduces the need for user input, which limits the chances of human error. The software can also be developed to be highly user-friendly as the user interfaces can be simplified to include only the functions that are necessary for the specific task. The user can also be directed through the process and adequately informed about the available options, thereby reducing the possibility of error.

The ease of use and increased integrity of SDSS make spatial analysis more accessible to users with very little or even no GIS skills. Unfortunately, the higher level of sophistication of SDSS comes at a price. Even if an organization is willing to invest in the development of a SDSS, the GIS licensing costs that are often involved impede its deployment. 


\subsection{THE INTERNET AND GIS COMMUNICATION}

The Internet is a publicly accessible worldwide system of interconnected computer networks. It is based on the packet-switching Advanced Research Projects Agency Network (ARPANET) created by the US Department of Defence in the early 1970s (Longley et al. 2002; Wikipedia 2005). Since the development of the World Wide Web (WWW) by Berners-Lee (1989), the uptake of WWW (or Web) technology has been remarkably quick. This hypertext-based service has brought the Internet into the realm of everyday use. In 2002 it was estimated that the Web consisted of two billion publicly-indexed web pages (netz-tipp.de 2002), while in a more recent study this figure was estimated to be 11.5 billion (Antonio \& Signorini 2005).

Since the early 1990s, the Internet has had a profound effect on technology, science and society. It has changed the way we conduct business, communicate, educate and govern (Longley et al. 2002). In the South African banking sector alone, more than one million online banking accounts existed in 2003. In 2004 the total increased by 32\% (Goldstuck 2004) and in 2006 by 49\% (Research Surveys 2006). Web-based automated information systems, such as online banking, enable companies to link their data-processing systems in an efficient and flexible manner. By doing so, companies can work more closely with suppliers and partners and better satisfy the needs and expectations of their customers.

The Internet is fast becoming a primary source of information and web users increasingly resort to it to support their decision making (Jarupathirun \& Zahedi 2007). DSS are being web-enabled owing to the increased accessibility and familiar interfaces of websites (Salewicza \& Nakayama 2004; Thysen \& Detlefsen 2006; Wang \& Chein 2003; Wang 2005) and because Internet technologies offer tighter integration with existing information systems (Vahidov \& Kersten 2004).

Concerning spatial technologies, the Internet has been the greatest external stimulus for GIS since the late 1990s. It has shifted the vision and basic role of GIS, i.e. to perform spatial tasks more efficiently, to communicating geographical information between users. The Internet provides an easy, cost-effective way to access spatial databases distributed worldwide. Greatly stimulated by market demand for geographical information, web applications that serve spatial data have grown rapidly since the first Internet mapping site was introduced by Xerox PARC in 1993. In 2002, more users made use of GIS functionality through the Internet than through all the other types of GIS software combined (Longley et al. 2002). 


\subsection{WEB MAPPING FOR SPATIAL DECISION SUPPORT}

Since its introduction in the middle to late 1990s, web mapping has been increasingly used to distribute geographical data over the Internet. Web mapping software such as Google Maps (Google 2005), MapMachine (National Geographic Society 2005), AlertNet (Reuters Foundation 2005), MapQuest (MapQuest 2008) and StreetMap (MWEB 2005), enables anyone with access to a computer and the Internet to explore geographical data online and produce maps on demand. With these tools, users can access geographical information without the need for expensive GIS software (Van Wyngaarden \& Waters 2007).

The introduction of products such as Google Earth has highlighted the value of web mapping for spatial decision support. Many organizations have recognized the potential of such systems for the cost-effective distribution of maps and other spatial information within organizational structures to improve productivity and to make better decisions. The functionality of most web mapping applications is however limited to data display and does not support GIS functionality such as editing, spatial analysis and modelling (Pummakarnchana, Tripathi \& Dutta 2005).

Web mapping solutions that offer more advanced functionality often require supplementary software to be installed on the user's computer. The installation of these so-called plug-ins and interpreters (Jiang 2003) is a deterrent to many web users who are not familiar with downloading and installing software. In addition, many users regard software downloads as a security risk. Consequently, for optimal accessibility, web-based SDSS should preferably be compatible with existing web browser software.

GIS functionality is difficult to implement using web technology due to the complexity of managing the data used, created and updated during these operations (Green \& Bossomaier 2001). However, the gap between GIS and web mapping applications is expected to close as the demand for more functionality increases. The addition of spatial analysis and modelling capabilities to web mapping applications holds much potential (Jiang 2003), especially for the cost-effective development of web-based spatial decision support systems.

\subsection{RESEARCH PROBLEM FORMULATION}

The research addresses two problem fields: firstly, the technical development needs of spatial decision support systems, and secondly the application of the technology towards a specific development challenge in the Western Cape Province. Both problem elements are encapsulated here. 
GIS are invaluable for supporting spatial decision making. GIS can be regarded as toolboxes with a large selection of operations that can be used for a wide range of applications. Unfortunately, the flexibility of GIS makes them difficult to use because operators not only need to know which tools are appropriate for a specific task, but they also need to know how each operation functions. The implication is that only those skilled in GIS have access to functionality such as spatial analysis and modelling. To make this functionality more accessible to decision makers, GIS are often customized into easily used SDSS. The development and deployment of SDSS have traditionally been expensive due to high development and/or software licensing costs.

Web mapping has established itself as a highly cost-effective means of communicating spatial information. Internet technology provides a possible solution to the high cost of SDSS, as it eliminates the need for expensive hardware or software. However, the problem exists that, due to the complexities involved, existing web mapping technologies do not offer the spatial analysis capabilities required by SDSS. This limitation currently impedes the use of the Internet for SDSS deployments. Consequently, one purpose of this research is to answer the following question: Can web mapping be extended with currently available technology to include the spatial analytical functionality required by SDSS?

The second problem element is embedded in the particular development challenges of the Western Cape Province - the area of application for this research. The province is experiencing an alarmingly high population growth rate of $2.86 \%$ which is the second highest of the nine South African provinces (Statistics South Africa 2001). Population growth, together with an urbanization level of 90\% (Kok \& Collinson 2006), are causing increasing needs for housing and food which place immense pressures on the province's land resources.

The Western Cape contributes 23\% towards the national agricultural contribution to GDP and agriculture is one of the major industries and the biggest user of land in the province. More than 11 million hectares $(84 \%)$ of the province's land surface is currently producing more than $55 \%$ of South Africa's total agricultural exports, of which the principal products are fruit (27\%), winter grain (21\%), white meat (18\%), wine (18\%) and vegetables (16\%) (CNDV Africa 2005).

Wine and fruit are the two principal export products of the Western Cape. Due to an everincreasing demand for quality wine and fruit, the land area under wine grapes and orchard cultivation is steadily expanding. Nationally, the total area under vineyards increased from 84 030ha in 1994 to 101 958ha in 2007 - an average annual expansion of nearly 2\%. Almost all (96\%) of the wine-grape vine plantings occurred in the Western Cape, placing increased pressure on existing land resources (SAWIS 2008). 
The Western Cape's natural resources cannot be managed sustainably without performing sound land use planning. Such planning requires accurate information about the suitability of land for specific purposes. Because of the size and variety of existing land uses in the Western Cape, more accessible and easily used tools are needed to support decisions about land use in the province. It is therefore a secondary challenge to this research to adapt and apply the developed spatial decision support system to demonstrate its applicability to implementation in the Western Cape Province.

Land suitability analysis is one of the first and arguably the most useful applications of spatial technology as it strongly relies on spatial analysis techniques (Malczewski 2006). In South Africa, and specifically in the Western Cape Province, there is a general lack of awareness among planners of the benefits and possibilities of SDSS for land use planning (Moss 2006, pers com). Most of the few SDSS that can be applied for land suitability analysis are prohibitively expensive, difficult to implement, user-unfriendly, or lack interactive spatial scenario-building capabilities. In addition, most systems require considerable resources of hardware, software and data (De la Rosa 2002; De la Rosa et al. 2004; Kalogirou 2002; Rossiter 2001; Rossiter \& Van Wambeke 1997).

No land evaluation systems are presently in use in the Western Cape. The only comparable systems actively in use are C-Plan and the Cape Environmental Management System (CEMS). C-Plan is being employed by the Cape Action for People and the Environment (CAPE) to aid conservation planning in the Cape floristic region (CFR) (New South Wales National Parks and Wildlife Service 2001; SANBI s.d.), whereas CEMS was developed for CapeNature to evaluate land for its conservation potential and to determine the effectiveness of existing nature reserves (CapeNature 2007; Van Niekerk 1997). Because both systems are only concerned with one land use, namely conservation, neither of them is a true land evaluation system. There is clearly an urgent need for a land evaluation system that can be used to support decisions about the Western Cape's stressed land resources.

\subsection{RESEARCH AIM AND OBJECTIVES}

The primary aim of this research is to evaluate the potential of the Internet to deliver low-cost, user-friendly and interactive spatial analysis functionality to a wide audience. To serve the widest possible audience, only technologies that are compatible with existing web browser software will be considered. A web-based SDSS will be developed to better understand the capabilities and limitations of the currently available technologies. Because SDSS are problemspecific, an application that adequately evaluates the spatial analysis capabilities of the SDSS is required. 
Hence, the secondary aim of this research is to build a web-based SDSS, called the Cape Land Use Expert System (CLUES), which can be used to perform land suitability analyses for the Western Cape Province.

To achieve the research aims, five objectives have been set:

1. Review the literature to determine the system and data requirements of a web-based land evaluation system and to overview the technologies now available.

2. Collect and prepare fundamental data sets for testing and demonstrating a web-based land evaluation system.

3. Design, develop and implement CLUES.

4. Demonstrate how CLUES can be used to create land use scenarios for the Western Cape.

5. Critically evaluate CLUES, make recommendations for its improvement and point out the limitations and potentials of Internet technology for SDSS development.

\subsection{THE STUDY REGION}

While the spatial application is of secondary importance only, some introductory background to the Western Cape as province is in order. The Western Cape is South Africa's fourth largest province, covering 11\% of the country's land area (see Figure 1-1). In 2007 the province accommodated approximately 4.8 million people, $10.1 \%$ of the national total (Statistics South Africa 2007). At $129462 \mathrm{~km}^{2}$ it is about the same size as England or Bangladesh.

The Western Cape is well known for its natural beauty and its environmental and biological diversity. It comprises most of the CFR, the only floral kingdom located entirely within the geographical confines of one country. The CFR is recognized globally as a biodiversity hotspot which covers only $0.05 \%$ of the earth's land surface, but as for biodiversity it contains three per cent of the world's plant species (SANBI s.d.).

Thanks to its Mediterranean climate and relatively fertile soils, agriculture is one of the main economic activities of the Western Cape. The province generates more than $20 \%$ of South Africa's gross farming income while employing one quarter of all the country's farm workers (Statistics South Africa 2006b).

Economically, the Western Cape has been booming, with an average real annual economic growth rate of $4.2 \%$ between 1996 and 2005. This is the highest of all the provinces and is considerably better than the national rate (3.7\%) over the same period. In 2005 the Western Cape's growth rate increased to 5.7\% (Statistics South Africa 2006a). 


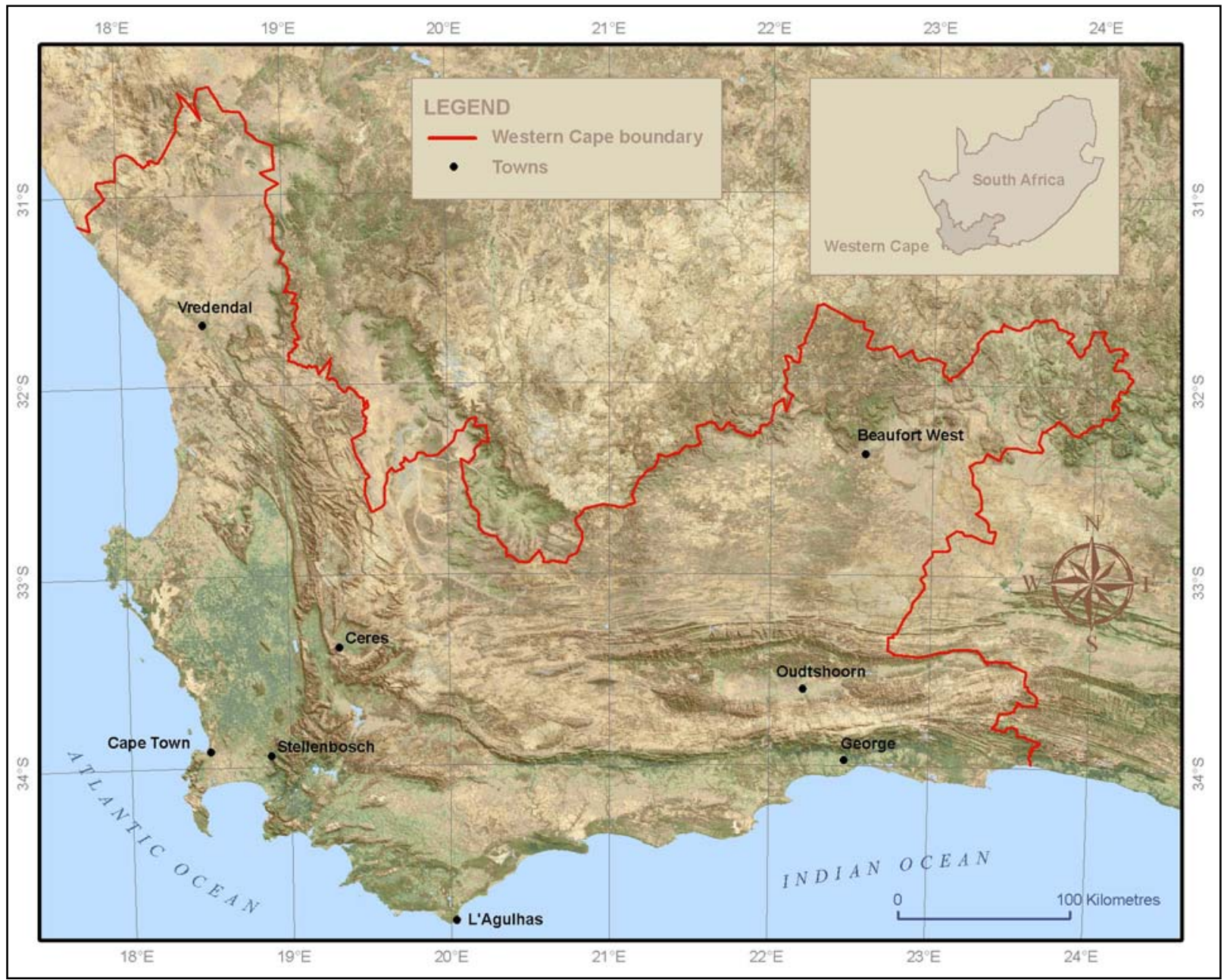

Figure 1-1 The Western Cape province, South Africa

\subsection{RESEARCH METHODOLOGY AND AGENDA}

This research is investigative and experimental in nature, with the intended outcome being a new online information system by which available Internet technology can be qualitatively evaluated for its applicability to land suitability analysis specifically and SDSS in general.

The research design is shown in Figure 1-2. The planning phase involves the identification and formulation of the problem, followed by setting the aims and objectives. These aspects have been dealt with in this chapter.

The second research activity is to conduct a literature review to establish what land suitability analysis entails. An outline of the land evaluation process and related concepts is provided in Chapter 2. The literature review also covers the various approaches to land suitability analysis, namely Boolean overlay, multi-criteria decision making and expert systems. Chapter 2 concludes with an overview of existing SDSS for land evaluation. 


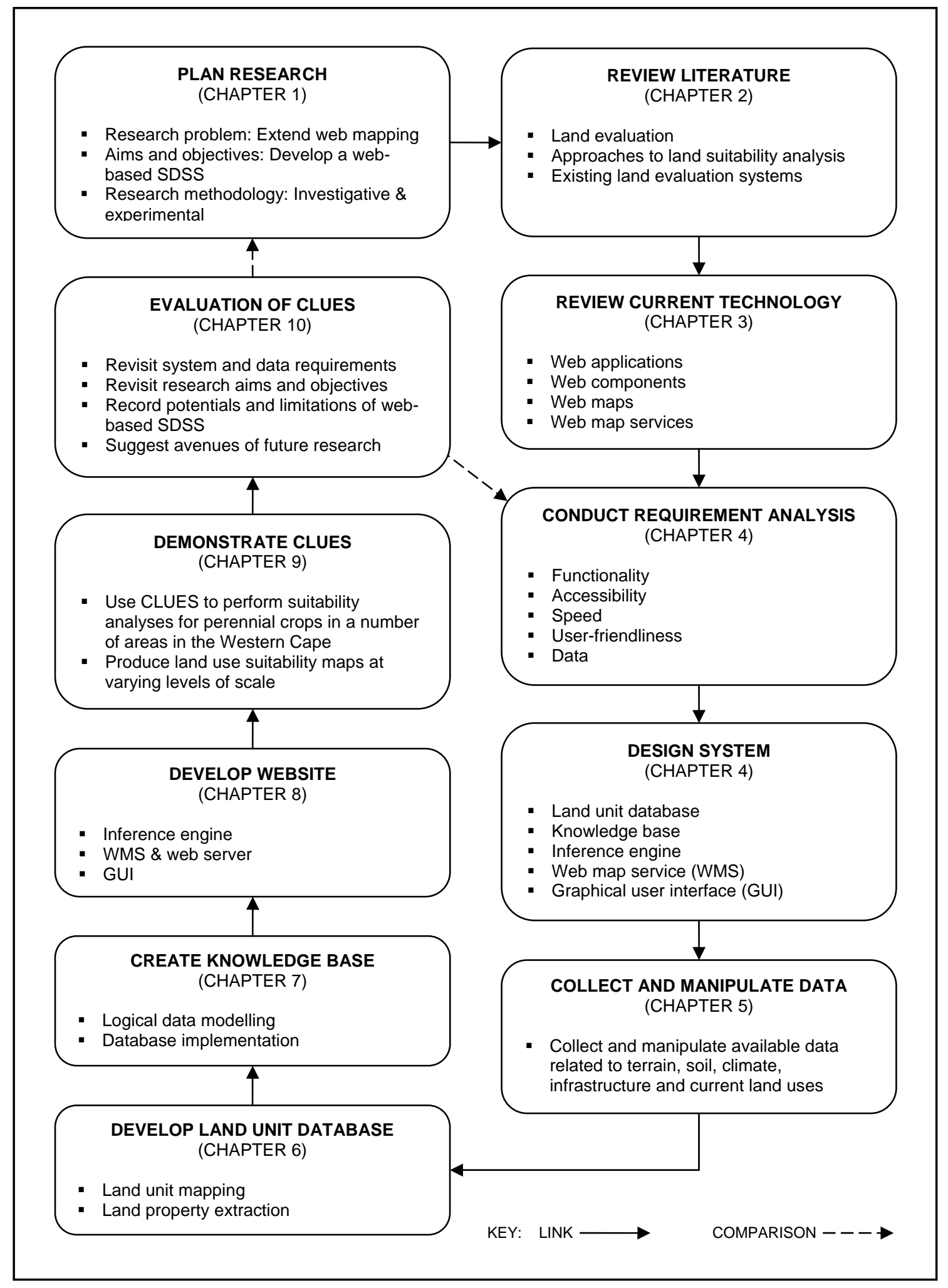

Figure 1-2 Research design for developing CLUES, a web-based SDSS for the Western Cape

Owing to the strong focus on technology in this research, Chapter 3 is a review of technologies now available for online spatial information system development. The review provides a basis for designing the web-based land evaluation system reported in Chapter 4. As with most software developments, this design phase was preceded by a requirement analysis to determine what functionality and other characteristics the system should exhibit. 
Owing to the intrinsically spatial nature of land suitability analysis, the types of geographical data to be analysed had to be considered in the system implementation. Chapter 5 describes the availability and collection of fundamental data sets on terrain, climate and soils. Activities related to the collection of the data and the manipulations needed to redress the lack of data are reported.

Data collection is followed by the implementation of the system, which comprises the creation of the three main components of the land evaluation expert system. The development of the first component, namely the land unit database, involves the mapping of the land units (i.e. basic mapping unit) and the extraction of land property data (i.e. environmental and physical information about land units). These two activities are discussed in Chapter 6.

The second component of CLUES is the knowledge base. As the name suggests, the knowledge base is a database that stores expert knowledge in the form of land use requirement rules. The design and implementation of the knowledge base, using the logical data modelling procedure, are described in Chapter 7.

The knowledge base not only contains rules, but also manages the operational data needed for the functioning of the CLUES website, as set out in Chapter 8. The website component consists of three parts, namely the inference engine, web map service (WMS) and graphical user interface (GUI). The function of the inference engine is to relate the information in the land unit database to the rules in the knowledge base to evaluate each land unit's suitability for a particular use. The suitability ratings are used to produce suitability maps, which are created and distributed through the WMS. The most prominent element of the CLUES website is the GUI, which directs user interaction with the system.

To demonstrate the functionality of CLUES, a number of land use scenarios are created and discussed in Chapter 9. This involves performing a suitability analysis for perennial crops and producing suitability maps at varying scales for two agricultural regions in the Western Cape. Each step is described in detail and illustrated with screen captures of the user interface.

Finally, in Chapter 10 CLUES is evaluated by comparing it with each of the requirements stipulated in Chapter 4. In addition, the research is critically assessed regarding the achievement of objectives. The potentials and limitations of web technology for SDSS deployment are discussed. The report concludes with some suggestions for further research.

In Chapter 2 which follows, the relevant literature is reviewed. 


\section{CHAPTER 2: THE PRINCIPLES AND PRACTICE OF LAND SUITABILITY ANALYSIS}

Physical land suitability analysis is a prerequisite for land use planning and development as it supports decisions regarding land use and leads to optimal use of land resources (Van Ranst et al. 1996). Owing to the vast quantities of data required in land suitability analysis, computer technology is often employed to manage, store, and analyse such data (Davidson 1986; De la Rosa et al. 2004; FAO 1984). Because most data used in suitability analysis is spatial in nature, geographical information systems (GIS) have become invaluable in land evaluation (Dai, Lee \& Zhang 2001). The GIS procedures in land suitability analysis can, however, be extremely timeconsuming and laborious processes without the aid of some level of automation. Fortunately, most modern GIS allow users to program the software to repeat a series of operations. This capability of GIS not only speeds up the process of suitability analysis, but also facilitates the creation of land use scenarios.

GIS that are customized for scenario building are often called spatial decision support systems (SDSS). SDSS are excellent platforms for land suitability analysis as different land suitability scenarios can be generated by making slight changes to the land use requirements or land properties. The true potential of SDSS, supported by GIS, lies in the ability to incorporate large spatial data sets so that local land use decisions can be made while considering the effects on a regional or provincial scale. The user-friendliness of SDSS also allows planners and decision makers, who are often incapable to use GIS, to perform land suitability assessments themselves.

The primary aim of this research is to develop a web-based Cape Land Use Expert System (CLUES) to demonstrate how the Internet can be used as a platform for SDSS and how it can make GIS functionality, in particular spatial analysis, more accessible and cost-effective. In this chapter, an outline of the land evaluation procedure is provided to illustrate what functionality is needed by such a system. This is followed by an overview of the three main approaches to developing land evaluation systems, namely Boolean overlay, multi-criteria decision making, and expert systems, as elements of each of these techniques are used in the system design.

\subsection{LAND EVALUATION APPROACHES}

Land evaluation is the interpretation of land properties such as climate, soils, fauna and flora in terms of the requirements of alternative land uses (FAO 1976). Land evaluation can therefore be defined as the process of estimating the potential of land for alternative uses (Dent \& Young 1981). Land evaluation, based on the guidelines set out by the Food and Agriculture Organization of the United Nations (FAO 1976; 1984; 1985), is an integral part of land use 
planning and has been established as one of the preferred methods to support sustainable land use management. Land evaluation gives an holistic, multi-disciplinary approach to sound development and conservation by combining economic and social principles with environmental, agricultural and biological sciences (Fourie 2006).

Land evaluation is based on the principle that certain land properties (i.e. soils, climate, topography and other environmental and social variables) influence the success of a particular land use. In essence, the objective is to compare and match each potential land use with the properties of each type of land (FAO 1984). The land evaluation process (Figure 2-1) involves eight interrelated steps: set objectives to be reached; collect appropriate spatial data; identify land uses to be considered; specify land use requirements; map land units; determine land properties; analyse the match between requirements and properties; and present results. These steps are described in the following sections.

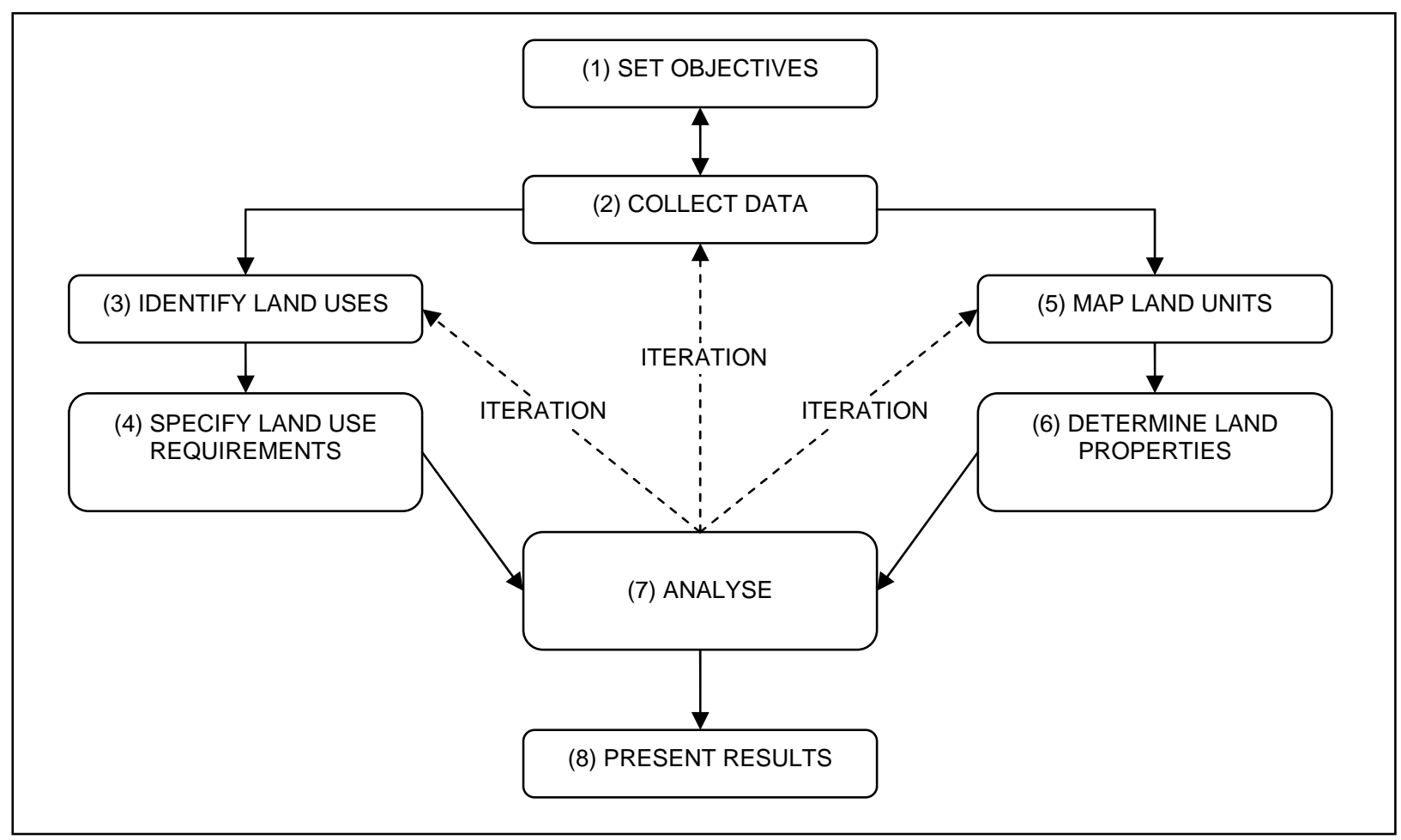

Figure 2-1 The steps in a land evaluation process

Adapted from Dent \& Young (1981)

\subsubsection{Set objectives}

The land evaluation process starts with a planning exercise in which the objectives of the evaluation are set. The most important decisions made at this stage are about the boundaries of the study area in which the evaluation will be carried out, and the level of detail required. The level of detail depends on the type of evaluation that will be done. Investigative and reconnaissance investigations are conducted at the largest map scale available to cover large (e.g. national or provincial) areas. The scales of such surveys vary from 1:2 000000 to 1:120 000 . 
Semi-detailed investigations are performed at scales ranging from 1:100 000 to 1:30 000, usually for smaller regions such as districts, municipalities or catchment areas. For applications at local or farm level, intensive and detailed investigations are needed. Such evaluations are usually carried out at scales larger than 1:10 000 (FAO 1976; Lambrechts \& Ellis s.d.).

Careful consideration is needed about the type of investigation envisaged because it determines how elaborate the evaluation will be and also dictates what data is required.

\subsubsection{Collect data}

The data requirements are determined during the second step of the evaluation process. This involves inventorying the available data to determine, if necessary, what additional data will have to be captured or purchased. If it is too costly to collect additional data, the evaluation objectives will have to be revisited. As most of the data used in land evaluation is spatial in nature, a GIS is often used to capture, store and prepare the data. Analysts must ensure that the data sets are of an appropriate scale and that they conform geographically (i.e. are registered and projected to the same coordinate system). Examples of phenomena captured in data might include topography, geology, soils, hydrology, vegetation, land use and climate parameters.

\subsubsection{Identify land uses}

Once the data has been collected, land uses that are worth considering for their suitability in the specified study area need to be identified. Land uses can be described in terms of 'major land uses' or 'land utilization types'. When an evaluation is done for a large area it is probably sufficient to specify broad or major land use types, such as rain-fed agriculture, irrigated agriculture, urban, and conservation areas. For more detailed studies, more specific and demanding subdivisions or land utilization types such as grains, deciduous fruit, residential, and wilderness area are more appropriate. Information about the production of goods (timber, crops or livestock) or the offering of services (recreational, sewage or refuse) is often used to describe land utilization types. Additional attributes to consider include market orientation, capital intensity, labour intensity, and type of technology employed. Commercial sugarcane production, on large privately owned properties, with low labour intensity, high capital inputs and level of mechanization, would be an example of a land utilization type (FAO 1985).

\subsubsection{Specify land use requirements}

In land evaluation, land is described according to land characteristics and land qualities. Land characteristics are properties of land that can be measured. Examples are slope gradient, slope aspect, soil depth and land cover. Several land characteristics can be combined to form a more 
complex land quality, such as fertility, available moisture supply or erodibility. Land qualities are often qualitative in nature, whereas land characteristics are usually quantitative (FAO 1976). Because either land characteristics or land qualities can be used to describe and classify land, the term land property is used in this research to encompass both terms.

The set of land properties needed to sustain a particular land use is called a land requirement. To determine land suitability, land properties are compared with land requirements (Burrough, MacMillan \& Van Deursen 1992), for instance the production of a certain crop needing deep, well-drained soils on gentle slopes, with an average annual rainfall of $300-500 \mathrm{~mm}$. In step five of the land evaluation process these requirements are specified for each of the land uses identified in step three (cf. Figure 2-1). Not only must the land use requirements be identified, but the values that will be considered suitable $(\mathrm{S})$ or not suitable $(\mathrm{N})$ should be specified. The principle classification of $\mathrm{S}$ and $\mathrm{N}$ is mainly based on technical, environmental or economic factors. In most cases a further classification is required to differentiate between highly suitable (S1), moderately suitable (S2), marginally suitable (S3), unsuitable at present (N1), and permanently unsuitable (N2).

\subsubsection{Map land units}

In step five of the land evaluation process, land units are demarcated. The term 'land' is often associated with any portion of the earth's surface not covered by oceans or water bodies, but the concept of land in the land evaluation context is much wider. Besides terrain and soil, land includes the total physical environment (e.g. climate, hydrology, vegetation) and the results of present and past human activity (e.g. salinization, vegetation clearance). For the purposes of land evaluation, social and economic characteristics are not included in the concept of land (FAO 1976).

Land units, or land-mapping units, are areas with properties that differ sufficiently from those of other land units to affect their suitability for different land uses. Although any parcel of land can be considered a land unit, it is more efficient and meaningful to use parcels that can be adequately described in terms of one or a combination of land properties. A land unit should therefore represent an area that is, in terms of predetermined properties, different from the surrounding land and can be assumed to have homogeneous land properties (FAO 1984). The degree of homogeneity or internal variation will vary depending on the scale and intensity set out in the evaluation objectives (FAO 1976). When a reconnaissance evaluation is carried out over a large region at a small map scale (i.e. 1:500 000 or smaller), large generalized land units such as climatic zones would be sufficient. For more detailed studies carried out at large map scales (i.e. 1:25 000 or larger), smaller map units such as soil types would be more appropriate. Landforms 
are often used as land units in medium-scale studies (1:25 000 to 1:500 000) because many physical land properties, including soil, climate and biology, are related to terrain (MacMillan, Jones \& McNabb 2004; Speight 1977). Examples of landforms and terrain units include crests, cliffs, terraces, footslopes, pediments, pediplains and alluvial plains (McDonald et al. 1984).

Although the size of the land units should be kept as small as possible to limit generalization, too many units can become unmanageable as each individual land unit is considered individually regarding its land properties and requirements. Fortunately, capacities to handle large numbers of land units have increased considerably with the use of computer technology and often the decision about the size, number and delineation of land units is determined by data availability. While soil type boundaries would probably be the most suitable delineation of land units for agricultural land uses, soil information is often not available at the required scales. In such cases other available data sets, such as landforms and terrain units, can be used instead.

\subsubsection{Determine land properties spatially}

The properties (defined in Section 2.1.4) of each land unit are determined spatially during step six. Using GIS, this essentially involves the conversion of vector land property data to raster format. Next, the land units are sequentially overlaid onto each raster to calculate the average land property values (e.g. annual rainfall, effective soil depth, slope gradient) for each land unit. The values are then added to the land unit layer as attributes.

\subsubsection{Analyse land units for suitability}

During analysis, each land unit's land properties are considered individually and compared with the land use requirements to classify a unit into its appropriate suitability level (i.e. S1, S2, S3, N1 or N2). Land suitability measurement can be as simple as determining whether a land unit meets all the land use requirements, or it might involve complex mathematical calculations to produce a suitability index used to find the optimal land use for a specific area. The chosen methodology depends on the required outcome and the classification method used when the land use requirements were set.

\subsubsection{Present results}

To conclude the evaluation process, the results are presented in the form of 'suitability' and 'solution' maps. Suitability maps are usually choropleth maps depicting the level of suitability of each land unit for a single land use using colour shading. Solution maps are simple qualitative thematic maps showing only the land uses that are most suitable for any given land unit. 
The land evaluation approach is versatile as it can be applied in rural or urban land use planning (Bosshard 2000; Dai, Lee \& Zhang 2001; Fourie 2006; Lütz \& Bastian 2002) and it can be done on national (Mantel et al. 2000), regional (Ceballos-Silva \& López-Blanco 2003b; Igué, Gaiser \& Stahr 2004), watershed (Nisar Ahamed, Rao \& Murthy 2000) or local (Cools, De Pauw \& Deckers 2002) levels. The procedure has been shown to be highly suitable for forestry (Thwaites \& Slater 2000; Twery et al. 2005), agricultural management (Dendgiz, Bayramin \& Yüksel 2003; Mantel, Zhang \& Zhang 2003; Mongkolsawat, Thirangoon \& Kuptawutinan 1997; Smith, McDonald \& Thwaites 2000; Wandahwa \& Van Ranst 1996) and conservation planning (Phua \& Minowa 2005).

Land evaluation is part of a larger land use planning process and the results should be used to support decisions about land use change. While land evaluation focuses on the suitability of land units for different uses, land use planning examines the relationships between uses. Factors such as social and economic needs of the community, as well as the environmental stability of an area, should also be considered during the planning process. Environmental conservation is always an objective of land evaluation and it is assumed that no form of land use will be judged suitable unless it can be sustained on a long-term basis without significant detriment to the land (FAO 1984).

It is obvious that suitability analyses lie at the centre of land evaluation and although computer processing is not a prerequisite for suitability analysis, it has become indispensable, especially where large numbers of land units are considered. The next three sections focus on the main approaches to land suitability analysis using computer technology, namely Boolean overlay, multi-criteria decision making, and expert systems.

\subsection{BOOLEAN OVERLAY}

Overlay procedures play a central role in land suitability mapping. Many agree that overlaying was introduced by McHarg (1969) when he superimposed individual transparent maps of natural and man-made environmental phenomena to produce overall land use suitability maps. This manual procedure was soon incorporated into GIS and has become one of their most useful operations.

The Boolean intersect and union operators are the two fundamental overlaying operations available in GIS and can be performed on raster or vector data. When raster data is used, a raster layer is required for each input feature (e.g. suitable soils) so that the position of features is represented by cell values of 1 where the feature is present, while cells where the features are absent have a value of 0 . When the Intersect operation is carried out on two Boolean layers, an 
AND comparison is made between the two layers. This means that if a cell has a value of 1 in both input layers, it will be given a value of 1 in the output layer. Cells that do not meet this requirement will be assigned a value of 0 . In the union operation, cells for which at least one input value is equal to 1 are awarded a value of 1 . In logical arithmetic this is equivalent to the OR operation. The output of the intersect and union operations is illustrated in Figure 2-2. In suitability analysis, Boolean layers are created by assessing each criterion's thresholds of suitability (Ceballos-Silva \& López-Blanco 2003a).

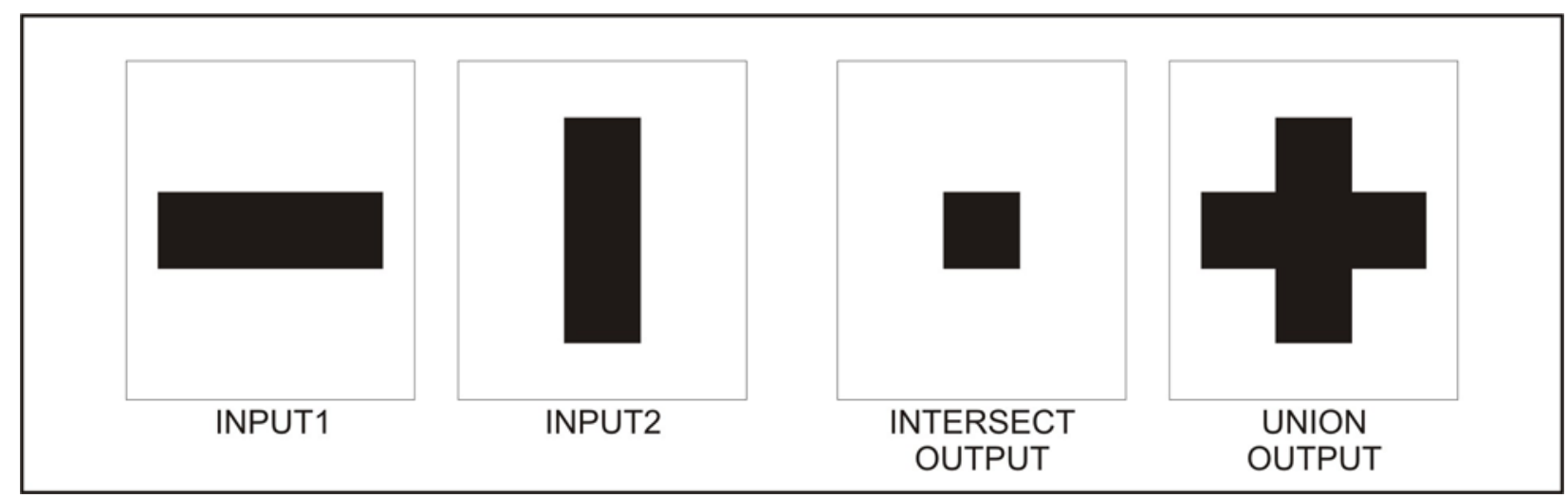

Figure 2-2 Intersect and union Boolean overlay operations

Land suitability often requires multiple input criteria. When Boolean overlay is used to analyse numerous input layers, two distinctly different results are obtained. Boolean intersection results in a very 'hard' decision as a region will be excluded from the result if any single criterion fails to meet its threshold. Conversely, the Boolean union operator implements a very liberal mode of aggregation: a region will be chosen in the result as long as a single criterion meets its threshold. By using the intersect operation the risk of producing an inaccurate classification is minimized as no trade-off between criteria is allowed (i.e. high suitability of one criterion cannot compensate for low suitability in another), while risk is maximized when the union operation is used as one criterion can override all other criteria (Ceballos-Silva \& López-Blanco 2003a).

Boolean overlay is popular among GIS users as it is a standard feature in most proprietary (offthe-shelf) GIS. Boolean layers (i.e. true/false binary layers) representing threshold values of land properties can easily be analysed using standard intersect (logical AND) overlay operators (Malczewski 2004). The operator used to combine criteria using Boolean overlay should be carefully considered as an inappropriate choice could result in inappropriate results. Another problem with Boolean overlay is that all criteria are of equal importance (Ceballos-Silva \& López-Blanco 2003a). Boolean overlay is also a very discrete or 'hard' decision strategy and this increases the risk of error due to inappropriate thresholds or inaccurate data (Eastman 2000). 
These limitations of Boolean overlay are addressed by multi-criteria decision making as discussed in the next section.

\subsection{MULTI-CRITERIA DECISION MAKING}

Decision making regarding land suitability is often difficult as it involves numerous stakeholders, multiple factors, and sometimes conflicting objectives (Traintaphyllou 2000). Because the alternative land uses for any particular parcel of land are potentially unlimited, many land use evaluation systems employ multi-criteria decision making (MCDM) techniques. MCDM (also referred to as multi-criteria evaluation (MCE) or multi-criteria analysis (MCA) in the literature) essentially divides a problem into smaller understandable parts and evaluates each part independently. The results of the individual evaluations are integrated to provide an overall solution to the original problem (Malczewski 1999). By using MCDM, solutions can be found to decision making problems with multiple alternatives, evaluated by decision criteria (Jankowiski \& Nyerges 2001).

The analyst can choose from a range of MCDM methodologies for a particular application. The available methodologies can be organized into three major dichotomies (Bester 2004):

- multi-objective versus multi-attribute decision problems;

- individual versus group decision makers; and

- decisions taken under certainty (deterministic) versus uncertainty (probabilistic and fuzzy).

Where multi-attribute decision making (MADM) produces alternatives based on attributes, multi-objective decision making (MODM) distinguishes between alternatives based on the objectives of the analysis. Because MODM requires alternatives it is usually executed as an optional additional step after MADM is completed (Malczewski 1999).

MADM and MODM can be performed by an individual or by a group. When a group of decision makers is involved, competitive or independent conflict can occur. Competitive conflict arises when preferences of different decision makers are in direct conflict, while independent conflict occurs when actions of one decision have indirect consequences on another (Eastman 2006; Malczewski 1999).

Decision making frequently involves a degree of uncertainty caused by inappropriate information, unforeseen circumstances, or invalid methods. To compensate for this, probabilistic or fuzzy approaches can be used instead of deterministic (Boolean) methods (Malczewski 1999). 
MCDM can support decisions concerning spatial and non-spatial problems. One spatial application for which it is routinely used is land suitability evaluation. The MCDM procedure is discussed in the next section.

\subsubsection{The MCDM procedure}

Van der Merwe (1997) suggests a seven-step procedure for applying MCDM to perform land suitability analysis (Figure 2-3). A brief overview of each step is provided in the following subsections.

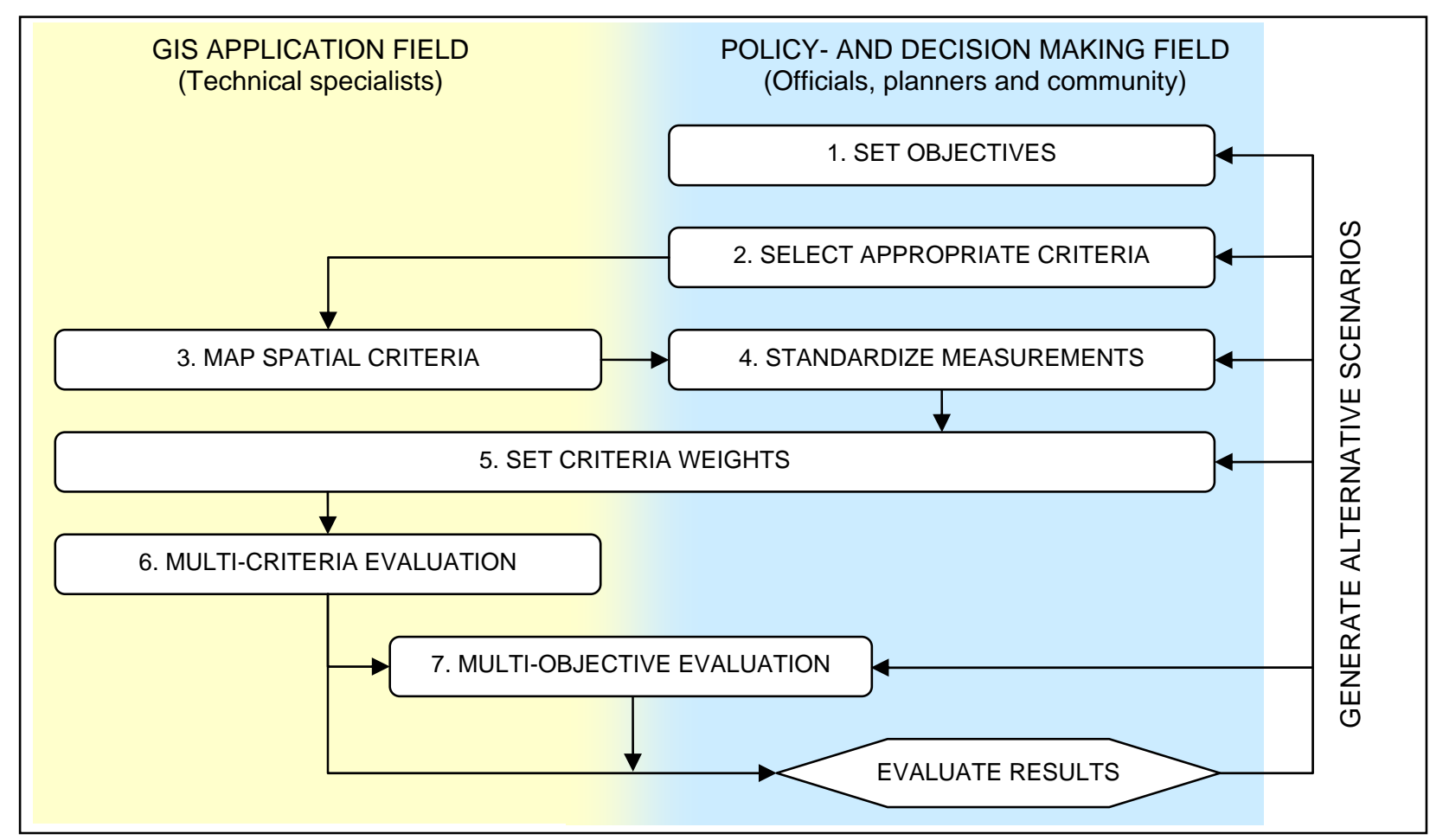

Adapted from Van der Merwe (1997)

Figure 2-3 A step-wise procedure for multi-criteria decision making

\subsubsection{Set objectives}

The objectives of the MCDM exercise must be set before the evaluation can be carried out as they dictate which methodology or decision strategy will be used in the evaluation (i.e. multiattribute, multi-objective, individual, participative, deterministic, and probabilistic). For instance, an objective might be to find areas most suitable for perennial crops, in which case a multiattribute evaluation would suffice as there is only one alternative (or land use). Another objective may be to find 100 hectares most suitable for perennial crops and 50 hectares most suitable for grain production: an objective calling for two land uses to be weighed against each other. Such an analysis requires multi-objective evaluation. 
If the requirements for each alternative are well established, the decision rules can be set by an individual expert. Group participation is often employed for semi-structured problem solving (Densham 1991). Due to the degree of uncertainty about data quality and precise land use requirement thresholds, probabilistic methods are the most frequently used, especially if multiobjective evaluation is required.

Land suitability analysis objectives should evolve from a problem statement based on discussions with stakeholders and/or a literature study. It is important that the study area and the scale at which the analysis will be carried out are clearly defined before the analysis is done. All the land uses considered in the evaluation must be specified, and assumptions and limitations due to data availability and time constraints should be acknowledged.

\subsubsection{Select appropriate criteria}

In step two of the MCDM process, the appropriate criteria for measuring land suitability are defined. Criteria can be either factors or constraints. Factors refer to criteria that enhance or detract from a land use's overall suitability, while constraints are meant to limit or exclude areas for consideration (Malczewski 1999).

\subsubsection{Map spatial criteria}

Once the criteria are selected, the factors and constraints for each criterion are mapped, usually using GIS. Due to the continuous nature of many criteria, a raster format is often chosen for MCDM. Depending on availability of existing data, new data may have to be captured. If existing data is used, reformatting and manipulation may be necessary as some GIS require raster data sets to have the same extent and resolution. Analysts must also ensure that the appropriate map projection and datum are used because criterion layers will be overlaid. Map projections that are true to area (i.e. equal- area projections) are recommended (DeMers 2005).

\subsubsection{Standardize measurements}

Once the spatial data is in the appropriate format, the level of suitability must be specified and incorporated into the data. This procedure of preparing the data for analysis is called measurement standardization. Because factors can be continuous and measured in different scales, step four of the MCE process requires all factors to be reformatted to a common measurement scale. To do so, linear scaling (Equation 2-1) is commonly used (Malczewski 1999).

$$
X_{i}=\frac{R_{i}-R_{\min }}{\left(R_{\max }-R_{\min }\right)} \times m
$$


where $\quad R_{i} \quad$ is the raw score;

$R_{\text {min }} \quad$ represents the minimum score;

$R_{\max } \quad$ is the maximum score; and

$m \quad$ is an arbitrary multiplier representing the upper standardized range value.

To demonstrate the use of Equation 2-1, it is known that perennial crops require soils of at least $300 \mathrm{~mm}$ deep and that suitability increases as soil depth increases due to the better resilience of perennial plants to withstand adverse climatic conditions. All soils with an effective depth of less than $300 \mathrm{~mm}$ can therefore be regarded as unsuitable (Schloms 2008, pers com). In this example, the parameters of Equation 2-1 are set to: $R_{\min }=300 ; R_{\max }=1200$; and $m=1$. Soils of $300-$ $1200 \mathrm{~mm}$ depth are rescaled to values ranging from 0 to 1 , with 1 representing the highest possible suitability and 0 the lowest. Figure 2-4 illustrates this example graphically.

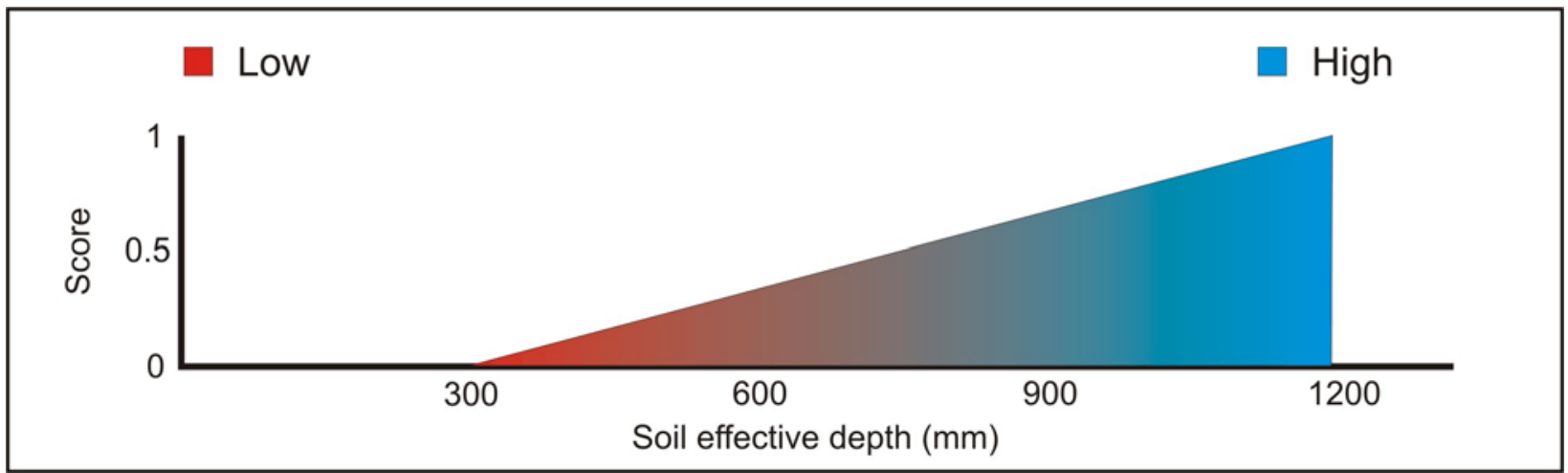

Figure 2-4 Linear scaling of effective soil depth for perennial crop suitability

Linear scaling can be regarded as a form of fuzzy classification as it incorporates a gradual transition between thresholds. Fuzzy classification is based on fuzzy set theory (Zadeh 1965) which resembles human reasoning when approximate information is used to make decisions. It was specifically developed to mathematically represent uncertainty and can be used to deal with the imprecision intrinsic to many spatial problems (Argialas 1995).

A fuzzy set can be defined mathematically as follows: if $X=[x]$ denotes a space of objects, then the fuzzy set $A$ in $X$ is the set of ordered pairs

$$
A=\left\{x, \mu_{A}(x)\right\} \quad x \in X
$$

where $\quad \mu_{A}(x) \quad$ is known as the 'grade of membership of $x$ in $A$ ' and

$x \in X \quad$ signifies that $x$ is contained in $X$. 
As with linear scaling, $\mu_{A}(x)$ is by definition a number ranging from 0 to 1 , with 1 representing full membership of the set and 0 non-membership. The level of membership of $x$ in $A$ does not represent probability but possibility: $\mu_{A}(x)$ of $x$ in $A$ specifies the degree to which $x$ belongs to $A$ (Burrough 1989; Sicat, Carranza \& Nidumolu 2005).

A fuzzy membership function (FMF) is used to determine the suitability value of a mapping unit. A popular FMF for land suitability analysis is the S-membership function (Huajun et al. 1991; Huajun \& Van Ranst 1992; Sicat, Carranza \& Nidumolu 2005) expressed in Equations 2-3 and 2-4. Graphic representations of S-membership functions are shown in Figures 2-5a and 2.5b. In Equation 2-3, when $x$ is equal to or exceeds $\gamma$, a full membership (i.e. $\mu(x ; \alpha, \beta, \gamma)=1$ ) is achieved. Lower values of $x$ will result in a partial membership (i.e. $S(x ; \alpha, \beta, \gamma)<1)$. Halfmembership (i.e. $\mu(x ; \alpha, \beta, \gamma)=0.5)$ is achieved when $x$ equals $\beta$ and non-membership (i.e. $S(x ; \alpha, \beta, \gamma)=0)$ is reached when $x$ is equal to or less than $\alpha$. Equation 2-4 is the descending version of Equation 2-3 and can be similarly described.

$$
\mu(x ; \alpha, \beta, \gamma)=\left\{\begin{array}{ll}
0, & x \in[-\infty, \alpha] \\
2[(x-\alpha) /(\gamma-\alpha)]^{2}, & x \in[\alpha, \beta] \\
1-2[(x-\beta) /(\gamma-\alpha)]^{2}, & x \in[\beta, \gamma] \\
1, & x \in[\gamma,+\infty]
\end{array}\right. \text { Equation 2-3 }
$$

where $\quad \alpha \quad$ is the lower limit of attribute $x$;

$\gamma \quad$ is the upper limit of attribute $x$; and

$\beta \quad$ is $(\alpha+\gamma) / 2$.

$\mu(x ; \alpha, \beta, \gamma)= \begin{cases}1, & x \in[-\infty, \alpha] \\ 1-2[(x-\alpha) /(\gamma-\alpha)]^{2}, & x \in[\alpha, \beta] \\ 2[(x-\beta) /(\gamma-\alpha)]^{2}, & x \in[\beta, \gamma] \\ 0, & x \in[\gamma,+\infty]\end{cases}$

where $\quad \alpha \quad$ is the upper limit of attribute $x$;

$\gamma \quad$ is the lower limit of attribute $x$; and

$\beta \quad$ is $(\alpha+\gamma) / 2$.

In the example of perennial crops an effective soil depth of at least $300 \mathrm{~mm}$ is required, while a depth of $900 \mathrm{~mm}$ or more is considered equally suitable. Equation 2-3 can be used in this scenario by setting $\alpha=300$ and $\gamma=900$ millimetres (see Figure 2-6a). In this case an asymmetrical function 

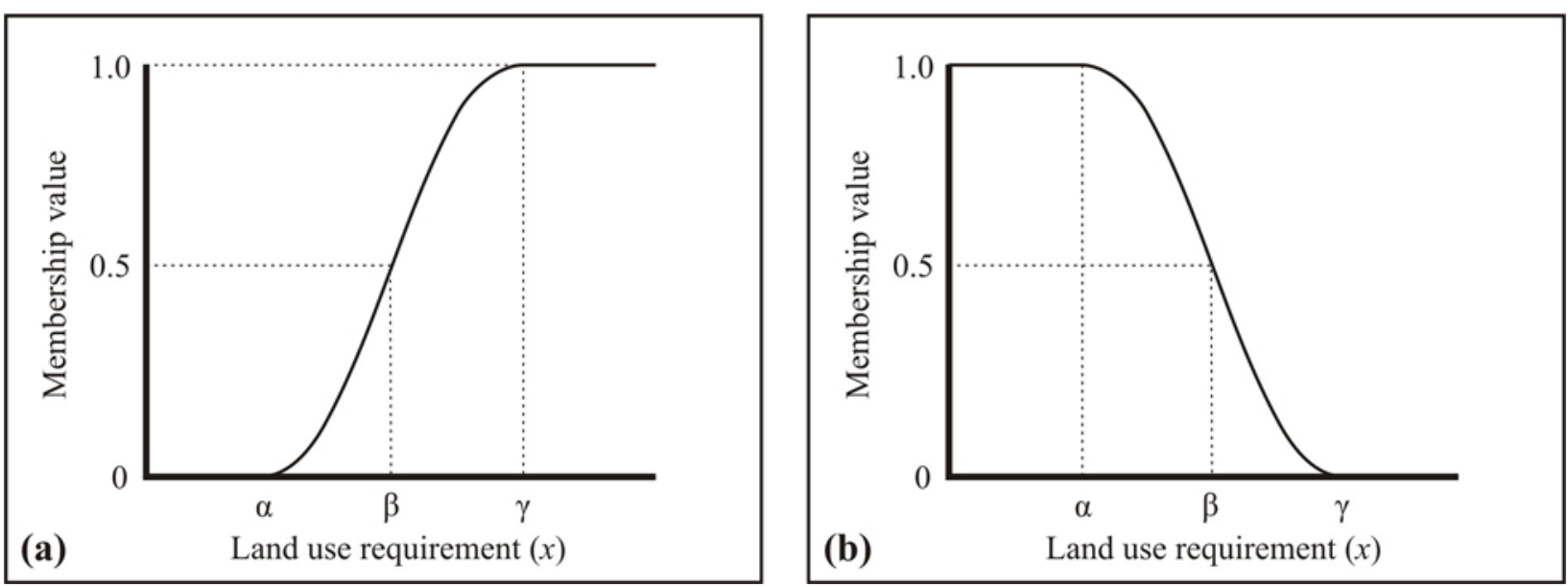

Figure 2-5 Ascending (a) and descending (b) S-membership functions

is more appropriate as perennial crops require soils having a pH between 5 and 7 (Fourie 2006). In this situation, the ascending and descending asymmetrical S-membership functions can be combined to form a symmetrical function (see Figure 2-6b) by setting $\alpha=5$ and $\gamma=6$ in Equation 2-3 and $\alpha=6$ and $\gamma=7$ in Equation 2-4.
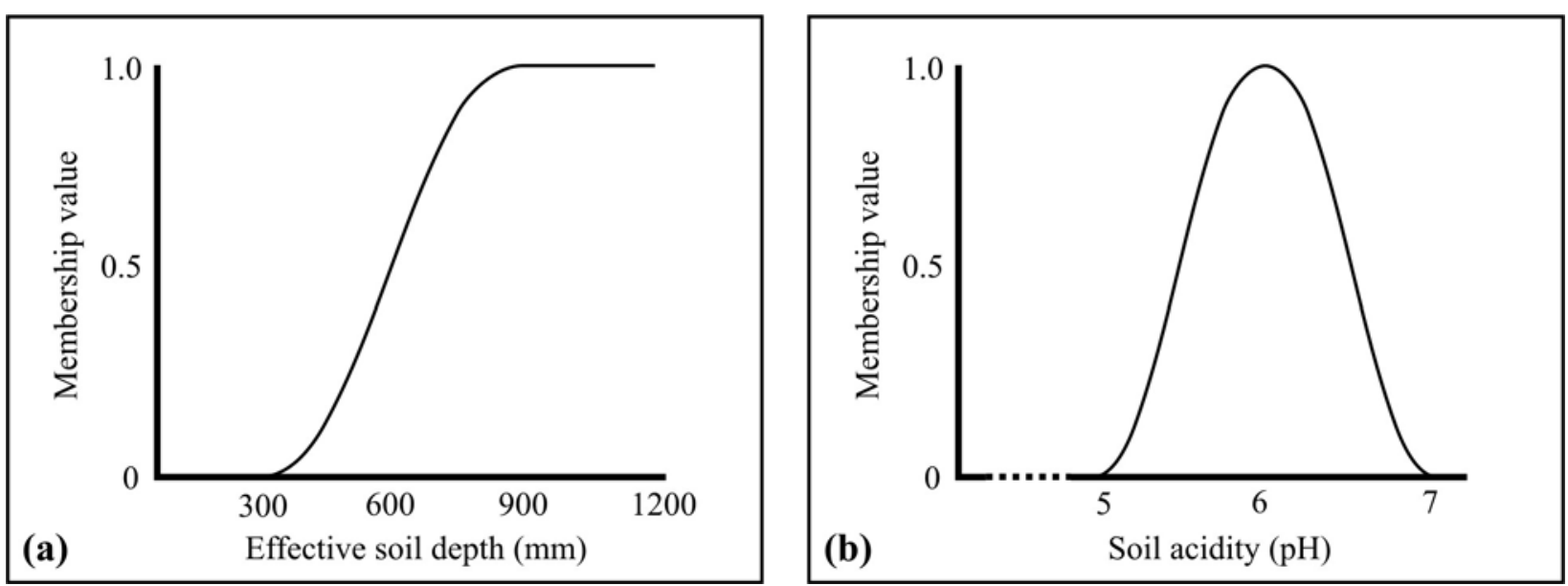

Figure 2-6 Effective soil depth (a) and soil acidity (b) membership functions for perennial crops

Huajun \& Van Ranst (1992) have shown that fuzzy methods are more accurate than Boolean classification. Another advantage of the fuzzy approach is its tolerance to inexact resource data. This is especially important in land evaluation as suitability analysts are often forced to use lowquality data or large mapping scales because no other data exists. To demonstrate so-called 'fuzzy tolerance', suppose the effective soil depth data in the perennial crops example is inaccurate and overestimates depths by 150 millimetres (i.e. a true depth of $300 \mathrm{~mm}$ is indicated as being $450 \mathrm{~mm}$ ). In this situation, a true depth of $375 \mathrm{~mm}$ is considered suitable using Boolean classification (compare Figure 2-7). In Equation 2-3, however, $\mu(375 ; 150,450,750)=0.03125$ indicating that the suitability of a depth of $375 \mathrm{~cm}$ is almost negligible in this scenario. 
The main disadvantage of fuzzy rules is their complexity. Setting the appropriate parameters for each requirement can be challenging, especially without specialized software to graph and visualize the effects of different parameters. In addition, programming is often necessary to implement fuzzy functions in GIS as more complex procedures are needed (Davidson, Theocharopoulos \& Bloksma 1994; Fourie 2006; Hall, Wang \& Subaryono 1992; Jiang \& Eastman 2000; Malczewski 2006; Nisar Ahamed, Rao \& Murthy 2000; Wang, Hall \& Subaryono 1990). Some software packages provide specialized tools using linear or non-linear functions to scale factors. The FUZZY module of IDRISI Andes is a good example (Eastman 2006).

In the perennial crops example, a constraint must be set for values of less than $300 \mathrm{~mm}$ (Figure 2-7) to prevent other factors (such as soil acidity) from compensating for effective soil depth. Constraints are given a suitability value of 0 and are represented by Boolean (or mask) layers (Eastman et al. 1995). All soils with a depth of less than $300 \mathrm{~mm}$ can therefore be regarded as unsuitable. Figure 2-7 graphically demonstrates Boolean classification of this threshold.

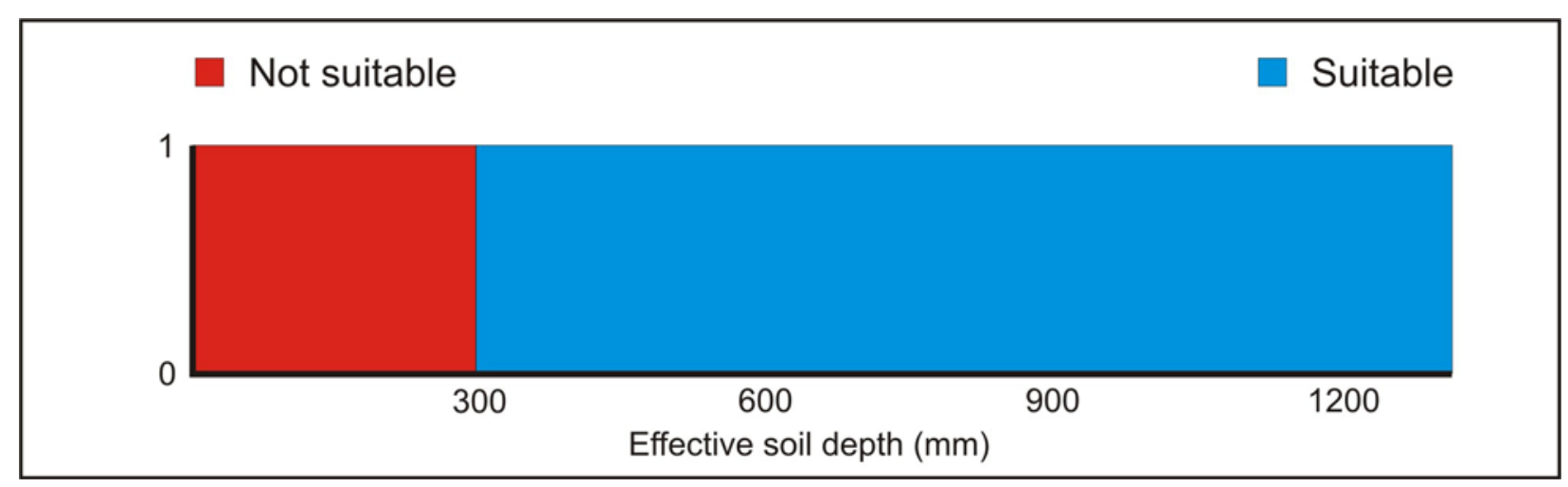

Figure 2-7 Boolean constraint of effective soil depth for perennial crops

Boolean classification is easy to implement using computer technology as simple logical (AND, OR) operations are required. Such operations are supported by structured query language (SQL), which is standard in most database management systems. Most GIS packages offer this functionality (Burrough, MacMillan \& Van Deursen 1992).

\subsubsection{Set criteria weights}

By nature different criteria do not have equal importance for a particular objective or land use. Effective soil depth might, for instance, be considered more important for wine grape production than slope gradient. To take this into consideration, each criterion must be weighted according to its relative importance. Weights can either be assigned by the analyst or in consultation with stakeholders. Weight values of criteria range from 0 to 1 and should be specified so that their sum is 1 . Deciding on which weights to allocate to each criterion becomes more difficult as the 
number of criteria increases. Fortunately, a method called the analytical hierarchy process (AHP) supports this task (Saaty \& Vargas 1991). AHP employs a pairwise comparison of criteria to arrive at a scale of preferences. Complex unstructured problems are broken down into their component parts, which are then arranged into hierarchical order. The relative importance of each pair of criteria is subjectively judged and numerical values (see Table 2-1) are assigned accordingly. These values are placed in a comparison matrix and evaluated.

Table 2-1 Scale of analytical hierarchy process (AHP) comparisons

\begin{tabular}{|c|l|}
\hline Numerical values & Description \\
\hline 1 & Equal importance \\
\hline 3 & Moderate importance \\
\hline 5 & Strong or essential importance \\
\hline 7 & Very strong or demonstrated importance \\
\hline 9 & Extreme importance \\
\hline $2,4,6,8$ & Intermediate values \\
\hline Reciprocals & Inverse comparison \\
\hline
\end{tabular}

Source Saaty \& Vargas (1991)

For instance, if effective soil depth, soil acidity, slope gradient and slope aspect are the criteria used to select the most suitable areas for perennial crop production, one might decide that effective soil depth is slightly more important than soil acidity. A value of 2 is given to this pairwise comparison and placed in the effective soil depth (row) and soil acidity (column) position of a comparison matrix (see Table 2-2).

Table 2-2 AHP comparison matrix for perennial crops

\begin{tabular}{|c|c|c|c|c|c|}
\hline & $\begin{array}{c}\text { Effective soil } \\
\text { depth }\end{array}$ & Soil acidity & Slope gradient & Slope aspect & Priorities (\%) \\
\hline Effective soil depth & $\mathbf{1}$ & $\mathbf{2}$ & $\mathbf{3}$ & $\mathbf{3}$ & 44 \\
\hline Soil acidity & $\mathbf{1 / 2}$ & $\mathbf{1}$ & $\mathbf{3}$ & $\mathbf{3}$ & 31 \\
\hline Slope gradient & $\mathbf{1 / 3}$ & $\mathbf{1 / 3}$ & $\mathbf{1}$ & $\mathbf{1 / 2}$ & 10 \\
\hline Slope aspect & $\mathbf{1 / 3}$ & $\mathbf{1 / 3}$ & $\mathbf{2}$ & $\mathbf{1}$ & 15 \\
\hline
\end{tabular}

Adapted from Saaty \& Vargas (1991)

If effective soil depth is slightly more important than soil acidity, it follows that the inverse is true (that soil acidity is slightly more important than effective soil depth) and a value $1 / 2$ is placed in the soil acidity (row) and effective soil depth (column) cell. Effective soil depth is also moderately more important than both slope gradient and slope aspect as indicated by the value of 3 in the appropriate cells. 
Once finalized, the comparison matrix can be used to determine the priorities of each criterion by using the AHP program (AHPP) developed by the Canadian Conservation Institute (2005). AHPP employs the principle Eigen value method as described in Saaty (1998) and was used to calculate the priorities for the example of perennial crops in Table 2-2. The resulting priorities are $44 \%, 31 \%, 10 \%, 15 \%$ for effective soil depth, soil acidity, slope gradient and slope aspect respectively.

Because comparison matrixes are created by human reasoning, they can contain inconsistencies. For instance, criterion A may be regarded as more important than criterion B, while B might be considered more important than criterion C. An inconsistency will occur if criterion $\mathrm{C}$ has been defined as being more important than criterion A (Marinoni 2004). To guard against such inconsistencies, Saaty (1977) introduced a consistency ratio (CR) which can be calculated from the principle eigenvector of the comparison matrix. A comparison matrix is considered inconsistent when its $\mathrm{CR}$ value is 0.1 or more. The CR for Table 2-2 is 0.045 according to AHPP, indicating that there are no significant logical inconsistencies present in the matrix.

AHP has been successfully applied in many MCDM applications and shown to be especially useful when public participation is incorporated in the weighting process (Mau-Crimmins, De Steiguer \& Dennis 2005). However, the setting of AHP scales can be confusing. According to Van der Merwe (2008, pers com) the pre-ranking of factors in order of importance can simplify the process considerably. Whichever method is used, many agree that the setting of weights without applying some kind of method to ensure consistency, could have adverse effects on suitability analyses (Malczewski 2004).

\subsubsection{Multi-criteria evaluation (MCE)}

In the sixth step of the MCDM procedure, the criteria are analysed to produce suitability maps. In MCE, factors, constraints and weights are combined using weighted linear combination (WLC). This essentially involves calculating a suitability value for a particular land use using Equation 2-5.

$$
S=\sum w_{i} x_{i} \times \prod c_{j}
$$

$$
\begin{aligned}
& \text { where } \quad \begin{array}{ll}
S & \text { is the suitability value; } \\
w_{i} & \text { is the weight of factor } i ; \\
x_{i} & \text { is the criterion score of factor } i ; \\
c_{j} & \text { is the Boolean criterion score of constraint } j ; \text { and }
\end{array}
\end{aligned}
$$


$\Pi \quad$ is the product of criteria.

In contrast to the high-risk Boolean intersect (AND) and union (OR) operations, WLC produces a risk-averse (Eastman 2000) and full trade-off solution (Mahini \& Gholamalifard 2006). If more control over the level of trade-off is required, ordered weighted averaging (OWA) can be applied as it employs an additional set of weights, called order weights, that are assigned on a location basis to manipulate trade-off (Malczewski 2006).

The result of MCE is a set of maps showing the level of suitability for each land use analysed. Suitability values are provided as a ratio scale from 0 to $m$ (see Equation 2-1). Graduated shades are often used to help visualize increasing suitability. Figure 2-8 demonstrates the result of a simple MCE involving two factors (A and B) of equal weight.

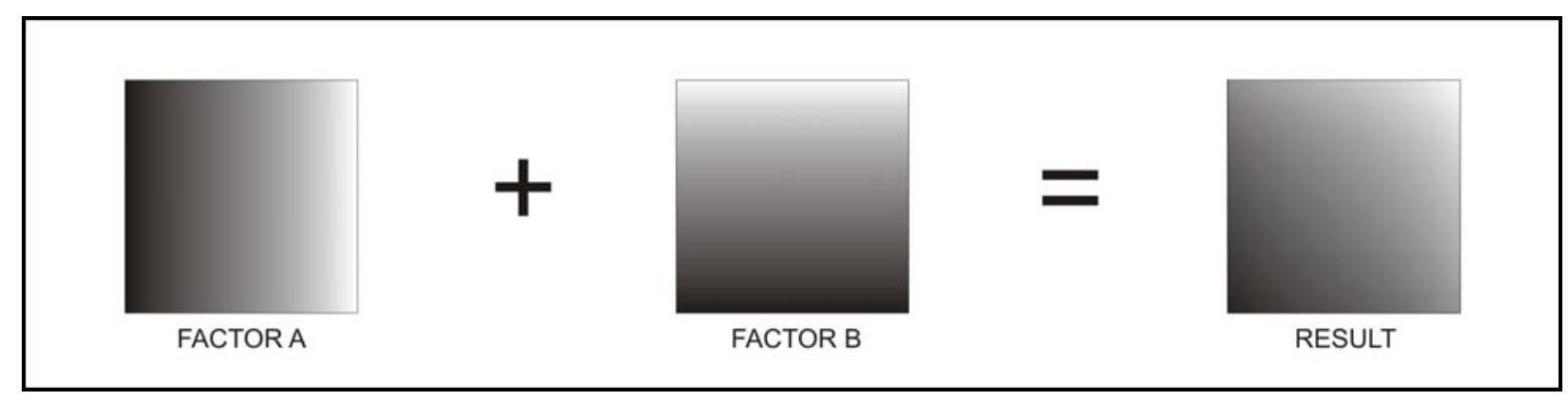

Figure 2-8 Graduated shades used to visualize suitability levels of factors and results

\subsubsection{Multi-objective evaluation (MOE)}

In multi-objective evaluation (MOE), alternative objectives (i.e. land uses) are compared to find the best solution according to the objectives set during the first step of the MCDM process. Land uses can either be conflicting or complementary (or non-conflicting). Conflicting land uses occur when a land parcel is suitable for two or more land uses, but can be used for only one purpose. If a parcel of land is suitable for more than one land use and can accommodate multiple uses (e.g. recreation and forestry), it is considered to be complementary (Eastman 2006).

\subsubsection{Discussion}

The use of MCDM for land suitability analysis is well established. Three recent applications are those by Van der Merwe \& Steyl (2005), Agrell, Stam \& Fischer (2004) and Ceballos-Silva \& López-Blanco (2003b). However, MCDM is not limited to solving land suitability problems. Other applications are in economics (Al-Najjar \& Alsyouf 2003), noise pollution (Van der Merwe \& Von Holdt 2006), forestry (Bruno et al. 2006; Varma, Ferguson \& Wild 2000), conservation (Phua \& Minowa 2005; Wood \& Dragicevic 2007), flood vulnerability (Yalcin \& Akyurek 2004), transportation (Vreeker, Nijkamp \& Ter Welle 2002) and tourism potential 
determining (Van der Merwe, Ferreira \& Van Niekerk 2008). Because various methods are available, no two implementations of MCDM are identical. This versatility and flexibility makes MCDM a remarkably popular spatial decision support methodology.

Most of the MCDM-based SDSS reported in the literature were not specifically developed for land evaluation applications. The exception is Agro-Ecological Zone for Windows (AEZWIN), developed by Fischer, Granat \& Makowski (1998). Although AEZWIN boasts an interactive and intuitive user interface, it lacks a spatial component. To use the system, the data must be prepared in a GIS, imported to AEZWIN for analysis, and then exported to a GIS for visual interpretation. Such loosely coupled approaches are not suitable for SDSS as they cannot provide an environment that enables interactive scenario building.

A recent approach is to integrate MCDM with GIS to improve interactivity. An example is MCDM_AV, which is an extension for ArcView GIS that allows users to carry out multi-criteria evaluation on both raster and vector data in an existing GIS environment (Bester 2004). The advantage of incorporating MCDM into an existing GIS is that less programming is needed because much of the functionality needed by MCDM is inherent in GIS. The disadvantage of this approach is that the user needs to own a copy of the GIS software which can be prohibitively expensive. The user must also be proficient in GIS in order to operate them in such applications.

GIS are often used to conduct MCDM owing to the formers' ability to spatially integrate and compare multiple geographically referenced data sets. Figure 2-3 highlights the importance of GIS in the MCDM procedure, with GIS being instrumental in four of the seven steps (i.e. map spatial criteria, set criteria weights, multi-criteria evaluation and multi-objective evaluation). Due to their complexity, GIS analyses should preferably be carried out by GIS experts. Practitioners involved in MCDM can be separated into two groups: those doing the GIS analyses (GIS analysts) and those making the policies and decisions (officials, planners, and community members). It is perceivable that a GIS analyst might not always have sufficient insight into the problem at hand. Good communication between the specialist and the decision makers is therefore essential for the MCDM process to be successful as misunderstandings can lead to incorrect and possibly conflicting results (Van der Merwe 1997).

Although MCDM is highly effective when participation by many stakeholders is required (MauCrimmins, De Steiguer \& Dennis 2005), it can also be useful in projects where the policy- or decision maker is an individual such as an official, planner or scientist. Decisions regarding the weights of the criteria can be based on studies reported in the literature, rather than on multiple stakeholder participation. If the decision maker is sufficiently competent in GIS, the entire procedure can be carried out by one person (Van der Merwe 2006). An advantage of an 
individual (or small group) approach is that the MCDM procedure becomes more explorative if the analyst can experiment with the weightings without having to consult many stakeholders. This means that different weighting schemes can easily be applied to interactively build scenarios. Interactive scenario building is essential for decision support, especially where objectives are vague and problems semi-structured (Clarke 1990). Special software is required to automate the MCDM process to enable interactive scenario building. Unfortunately, the MCDM process is not easily automated because it constantly requires input from the analyst or decision makers. A more effective method is to allow the operator to set criteria standardization rules and weights once during each suitability analysis so that all the other steps can be automated. The setting of rules not only reduces user input, but also facilitates the storage of decisions for future reference and use. Such a rule-based approach is explored in the next section.

MCDM provides a highly flexible methodology for land suitability analysis. The wide range of methods available in MCDM can be confusing to some decision makers, especially if they are unfamiliar with the fundamental concepts. Using an inappropriate method can considerably influence the outcome of the evaluation (Joerin, Theriault \& Musy 2001). Care must be exercised when suitability values resulting from MCE are interpreted (Proctor \& Qureshi 2005). For practical implementations, suitability values are often converted from a ratio scale to an ordinal scale to produce more user-friendly suitability levels (i.e. low, medium, high). To do so, the analyst and/or stakeholders set minimum and maximum limits for each suitability class. Due to the way in which MCDM standardizes and combines criteria, the thresholds are often set without any consideration of the underlying factors and may therefore be inconsistent with the original criteria settings.

\subsection{EXPERT SYSTEMS}

Expert systems are computer systems that emulate human decision making (often called artificial intelligence) by considering a set of predefined rules. The main components of an expert system are a knowledge base which stores information in the form of rules, and an inference engine that contains protocols about how the rules in the knowledge base are applied (Encyclopaedia Britannica 2007).

The rules in the knowledge base are usually obtained by interviewing experts in a particular subject. The interviewer, or knowledge engineer, organizes the information obtained from the experts into a collection of rules, typically in an 'if-then' structure. The inference engine is then used to make deductions from the rules to solve complex problems (Encyclopaedia Britannica 2007). 
The ability of expert systems to support problems involving many different factors, makes it highly suitable for SDSS. Together with the capabilities of GIS to store, manipulate, analyse and present spatial data, expert systems are powerful tools for supporting complex spatial decisions such as the optimal use of land. Because land use requirements can be formulated easily as 'ifthen' rules, the rule-based approach of expert systems is eminently appropriate for land suitability analysis. For instance, if a land unit has an effective soil depth of more than $300 \mathrm{~mm}$, it can be regarded as suitable for the production of perennial crops. By applying this rule to the effective soil depths of all land parcels (i.e. land units) in a GIS database, the system can select all the land units that meet this requirement. Similar rules can be created for other land properties and combined using a predetermined methodology (i.e. inference engine) to calculate a suitability index.

While the requirements for some land uses are well researched, many land use requirements are unknown. In such cases, land management decisions are often made by experts like agricultural consultants, farmers or engineers. By capturing and safeguarding expert knowledge and experience in a database of rules, computers can be used to carry out land use evaluation over very large areas.

The expert system procedure for doing a suitability analysis is shown in Figure 2-9.

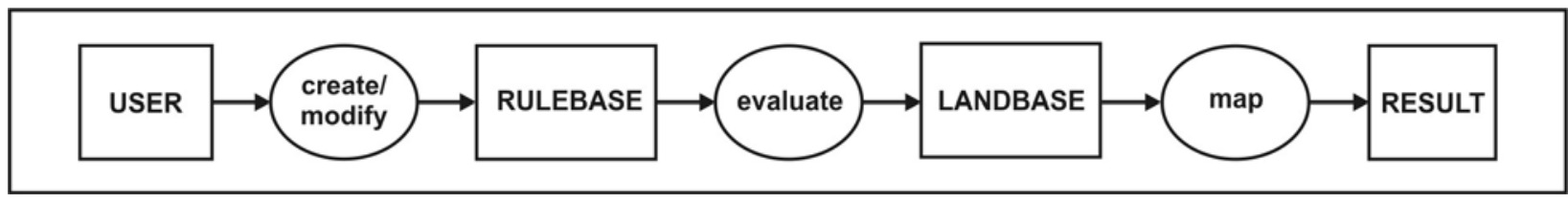

Figure 2-9 Procedure for an expert system land suitability analysis

The process starts with the establishment of land-requirement rules, which are then evaluated against the land unit properties. During evaluation, the inference engine assigns a suitability rating to each land unit, which is used to produce a suitability map. Different scenarios can be generated by repeating the process with altered rule sets. The subsequent sections consider the rulebase, inference engine and land unit data base and overview some existing systems.

\subsubsection{The rulebase}

Land use requirement rules are central to the expert system analysis procedure. Both Boolean and fuzzy rules are usually accommodated to provide as much flexibility in rule creation as possible (Encyclopaedia Britannica 2007). For Boolean rules, simple thresholds are used to define land property suitability. Soils can, for instance, be reclassified into binary values ( 0 and 1), where a value of 1 represents suitable, and 0 unsuitable soils (see Figure 2-7). To implement 
levels of suitability (i.e. S1, S2, S3, N1, N2), a set of thresholds can be specified as illustrated in Figure 2-10.

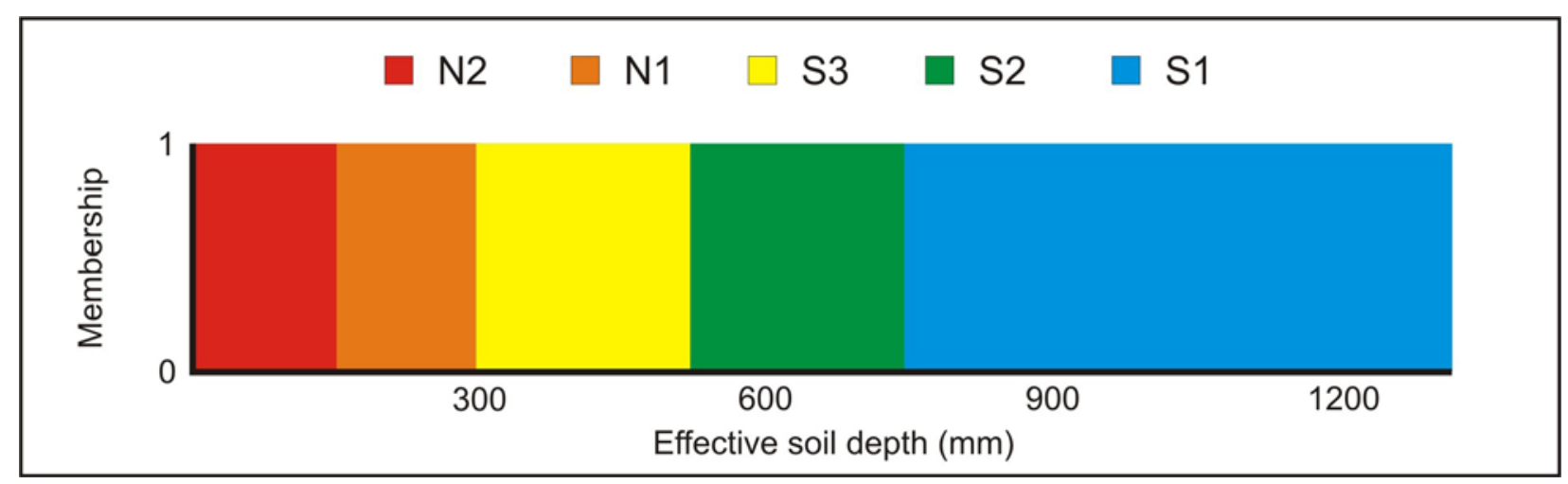

Figure 2-10 Levels of suitability of effective soil depths for perennial crops using Boolean classification

The individual threshold values needed to implement the land requirement shown in Figure 2-10 are provided in Table 2-3.

Table 2-3 Example of five Boolean rules specifying effective soil depth requirements for perennial crops

\begin{tabular}{|c|c|c|c|}
\hline$\#$ & SUITABILITY LEVEL & LOWER (mm) & UPPER (mm) \\
\hline 1 & Permanently unsuitable (N2) & 150 \\
\hline 2 & Unsuitable at present (N1) & 300 & 300 \\
\hline 3 & Marginally suitable (S3) & 525 & 750 \\
\hline 4 & Moderately suitable (S2) & 750 & 1350 \\
\hline 5 & Highly suitable (S1) & & \multirow{2}{*}{5} \\
\hline
\end{tabular}

For rule 1 the lower and upper thresholds are specified as $0 \mathrm{~mm}$ and $150 \mathrm{~mm}$ respectively and the lower threshold for rule 2 is set equal to the upper threshold of rule 1. A similar procedure is followed for the rest of the suitability levels. Because all soils of $750 \mathrm{~mm}$ or more are considered in this example to be highly suitable, the upper value of this suitability class should actually be infinity. To represent such 'open-ended' rules, the upper threshold is set to the maximum effective soil depth value in the database, which is $1350 \mathrm{~mm}$ in this case.

In reality, suitability increases as effective soil depth increases. In Figure 2-10, 300mm-deep soils are regarded just as suitable as $450 \mathrm{~mm}$-deep soils. A fuzzy set function (see Section 2.3.1.4) therefore better represents the effective soil depth requirement. To implement different levels of suitability, multiple fuzzy set functions are required. For instance, a total of seven S-membership functions are used in Figure 2-11 and parameters $\alpha$ (lower threshold) and $\gamma$ (upper threshold) are set so that levels overlap. In this example effective soil depths of $300 \mathrm{~mm}$ are regarded as either marginally suitable (S3) or not suitable at present (N1). As depths increase from 300mm 


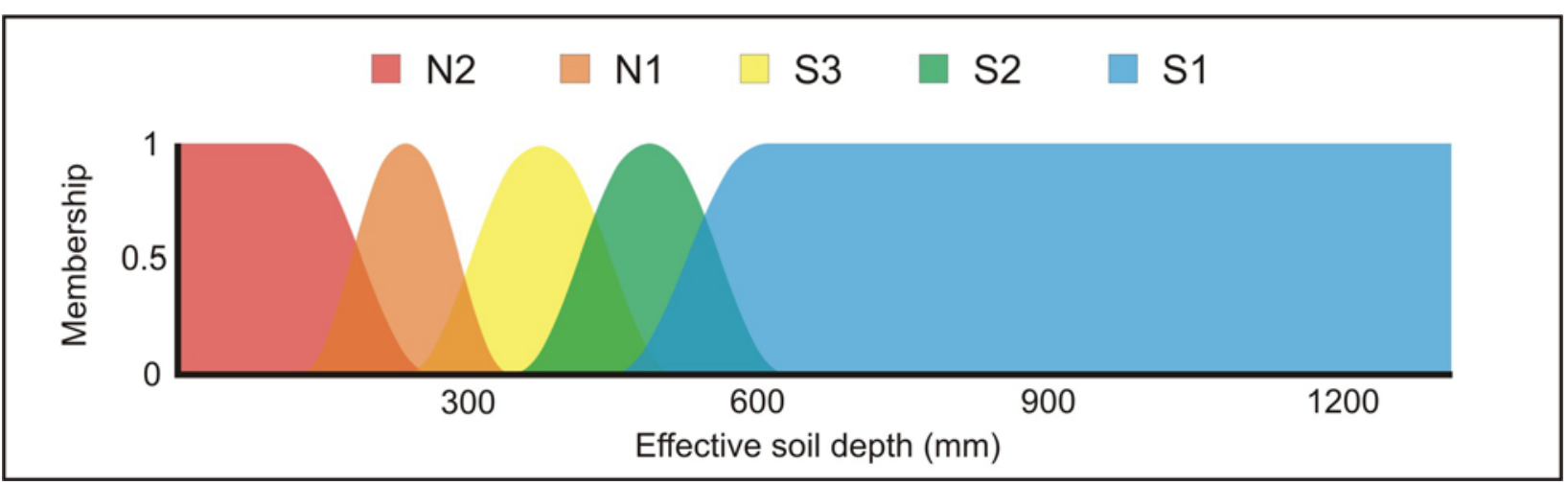

Figure 2-11 Levels of suitability of effective soil depths for perennial crops using fuzzy classification

to $450 \mathrm{~mm}$ the suitability value of level S3 increases, while the fuzzy value of N1 decreases. This provides a 'softer' transition between suitability levels when compared to Boolean classification (see Figure 2-10).

The rules needed to implement the example in Figure 2-11 are provided in Table 2-4.

Table 2-4 Example of two Boolean and five fuzzy rules specifying effective soil depth requirements for perennial crops

\begin{tabular}{|c|c|c|c|c|c|}
\hline$\#$ & SUITABILITY LEVEL & $\begin{array}{c}\alpha \\
\text { (lower threshold) }\end{array}$ & $\begin{array}{c}Y \\
\text { (central value) }\end{array}$ & RULE TYPE \\
\hline 1 & Permanently unsuitable (N2) & 0 & - & 150 & Boolean \\
\hline 2 & Permanently unsuitable (N2) & 150 & 150 & 250 & $\begin{array}{c}\text { Fuzzy } \\
\text { (asymmetrical) }\end{array}$ \\
\hline 3 & Unsuitable at present (N1) & 150 & 240 & 330 & Fuzzy (symmetrical) \\
\hline 4 & Marginally suitable (S3) & 250 & 390 & 520 & Fuzzy (symmetrical) \\
\hline 5 & Moderately suitable (S2) & 330 & 470 & 620 & Fuzzy (symmetrical) \\
\hline 6 & Highly suitable (S1) & 450 & 600 & 600 & Fuzzy \\
(asymmetrical) \\
\hline 7
\end{tabular}

For symmetrical fuzzy rules, an additional central value $(\beta)$ needs to be specified to indicate the centre of the membership function. For instance, in rule 4 membership increases from $250 \mathrm{~mm}$ and reaches a maximum membership at $\beta$ (i.e. $390 \mathrm{~mm}$ ). The membership value then decreases with increasing depth until it reaches $520 \mathrm{~mm}$ effective soil depth. For asymmetrical functions, the central value is set equal to $\alpha$ or $\gamma$, depending on the slope direction of the function. Rule 6 , for instance, has a positive slope (i.e. membership increases as effective soil depth increases) and is specified by setting the central value equal to $\gamma$ (i.e. $600 \mathrm{~mm}$ ). 


\subsubsection{The inference engine}

All seven rules in Table 2-4 constitute one land use requirement (effective soil depth) for perennial crops. Because the success of most land uses will depend on multiple requirements, each land property value $p_{i}$ must be sequentially tested against the fuzzy set function $S(x ; \alpha, \beta, \gamma)$ of each requirement $r_{i}$ by setting $x=p_{i}$ in Equation 2-3 and Equation 2-4. The resulting membership values are combined and averaged using Equation 2-6.

$$
S_{j}=\frac{\sum \mu_{i}\left(p_{i} ; \alpha, \beta, \gamma\right)}{n}
$$

where

$$
\begin{array}{ll}
S_{j} & \text { is the overall suitability value for land unit } j \\
\mu_{i} & \text { is the membership value for land property } p_{i} ; \\
\alpha & \text { is the upper limit of land requirement } \mathrm{r}_{i} ; \\
\beta & \text { is the central value; } \\
\gamma & \text { is the lower limit of land requirement } \mathrm{r}_{i} ; \text { and } \\
n & \text { is the number of land properties. }
\end{array}
$$

The result is an overall suitability value ranging from 0 to 1 . In Figure 2-6, if $p_{1}$ is effective soil depth and $p_{2}$ soil acidity of a particular land unit $u_{j}$, with $p_{1}$ set equal to $600 \mathrm{~mm}$ and the $\mathrm{pH}$ of $p_{2}$ set to 6 , then $S_{j}=(0.5+1.0) / 2=0.75$. To differentiate between the importance of land properties, weightings can be set and multiplied by individual membership values as expressed in Equation 2-7.

$$
S_{j}=\sum w_{i} \mu_{i}\left(p_{i} ; \alpha, \beta, \gamma\right)
$$

Equation 2-7

$\begin{array}{ll}S & \text { is the overall suitability value for land unit } j ; \\ w_{i} & \text { is the weight of land property } p_{i} ; \\ \mu_{i} & \text { is the membership value of land property } p_{i} ; \\ \alpha & \text { is the upper limit of land requirement } r_{i} ; \\ \beta & \text { is the central value; and } \\ \gamma & \text { is the lower limit of land requirement } r_{i} .\end{array}$

Suppose that effective soil depth $\left(p_{1}\right)$ in Figure 2-6 is twice as important as soil acidity $\left(p_{2}\right)$ for perennial crop suitability and that $p_{1}$ is equal to $600 \mathrm{~mm}$ and the $\mathrm{pH}$ for $p_{2}$ is 6 , then $S_{j}=$ $((0.66 * 0.5)+(0.33 * 1.0))=0.66$. It is important that the land property weights sum to 1 and that they are consistent when compared in pairs. To ensure consistency, Saaty’s (1977) analytical hierarchy process (AHP) is recommended when weighting land properties (refer to Section 2.3.1.4). 
Figure 2-11 shows how the upper and lower limits of several membership functions can be manipulated to represent the different suitability levels (S1, S2, S3, N1, N2). Suitability values must be calculated for each level using Equation 2-7. The level with the highest suitability value is identified and used to classify the land unit into that suitability level. In addition, to reclassify land units into suitability levels, the suitability values of different land uses can be compared to determine the most suitable land use for a particular land unit.

\subsubsection{Land unit database}

In the expert system approach, land units are usually stored as vector polygons in a GIS database. The vector data model is preferable because multiple attributes (i.e. land properties) can be linked to each land unit. This method is efficient because the geometrical properties (i.e. boundaries) of each land unit are stored only once, saving space and processing time. Another advantage of storing land properties as attributes to polygons is that suitability analysis merely involves the comparison of different fields in the database, which means that suitability values can be calculated by performing simple tabular operations. Although the use of tabular operations instead of spatial operations (i.e. overlaying) has significant benefits concerning processing speed, the main advantage of such operations is that the entire suitability analysis can be performed using standard database management operations (see Section 3.2.6). This means that GIS software is not required for the analysis part of the procedure. The only step in the expert system procedure involving spatial functionality is the mapping of the resulting suitability levels. Mapping is, however, a relatively simple operation and can be incorporated without the use of expensive GIS software. The next section overviews how mapping is handled in existing expert systems for land suitability analysis.

\subsubsection{Existing land evaluation systems}

The Automated Land Evaluation System (ALES) is one of the first and most popular land evaluation expert systems in existence. ALES, developed at Cornell University (Rossiter 2001; Rossiter \& Van Wambeke 1997), is an expert system based on Microsoft's Disk Operating System (MS-DOS) that allows users to build a database of rules to evaluate land according to the methods presented by the Framework for land evaluation (FAO 1976). The system includes a framework for a knowledge base, a land unit database and an inference mechanism that relates the knowledge base to the land unit database (Rossiter 2001).

ALES has been applied in thirteen cases of which the most recent examples include applications for land use planning in the Philippines (Lantican et al. 1998), the potential of sustainable wheat production in Uruguay (Mantel et al. 2000), banana production potential on Hainan island, China 
(Mantel, Zhang \& Zhang 2003) and for finding alternative uses for forested land (Fernandez Ruiz 2003). The frequency of appearance of publications in which ALES is used is declining, most probably because the last version (4.65) was released in 1996. A major drawback of ALES is that it is programmed in MS-DOS, for which support was discontinued with the release of Windows 2000. ALES also lacks a user-friendly graphical interface, which makes its implementation difficult. Another disadvantage is that an external GIS is required to view the results, which reduces the ability of the system to interactively produce different land use scenarios.

Another DSS developed since the early 1990s is the Mediterranean Land Evaluation Information System (MicroLEIS) which has evolved into an agro-ecological decision support system with a range of tools for land use decision making. It focuses on soil protection by improving agricultural soil use, its planning and its management. The toolkit includes a database, statistics, expert system and a neural network. As with ALES, the original MicroLEIS version was nonspatial, which means that a separate GIS was needed to input data and display results. Fortunately, a GIS version has recently been implemented in ArcView GIS to overcome this limitation. Another useful development in MicroLEIS is the establishment of a website to which users can upload tabular data, carry out suitability analysis, and download the results for visualization on any GIS. In spite of its lack of an integrated spatial component, the website is reportedly very popular with more than seven hundred registered users in 2004 (De la Rosa 2002; De la Rosa et al. 2004).

An expert system called the Intelligent System for Land Evaluation (ISLE) was developed in Borland Delphi as an MS Windows-based stand-alone system. The ISLE components include a user-friendly graphical interface, an existing FLEX expert system and the Borland database engine (BDE). Meanwhile, a major improvement over ALES has been the use of ESRI's MapObjects to include a fully interactive mapping component, allowing users to easily generate different scenarios and to see the results spatially (Tsoumakas \& Vlahavas 1999). MapObjects is a powerful collection of embeddable mapping and GIS components which developers can use to create applications that include dynamic maps and GIS capabilities (ESRI 2002c).

MapObjects was also applied in a SDSS called LEIGIS (Land Evaluation using an Intelligent Geographical Information System) to provide interactive mapping functionality. LEIGIS is similar to ISLE in most aspects, except that it was developed in MS Visual Basic and it uses the CLIPS expert system instead of FLEX (Kalogirou 2002).

The literature review indicates a distinct movement towards fully integrated systems that incorporate the required spatial functionality without the reliance on GIS software packages. The 
main advantage of such systems is that access is not limited to existing GIS users. However, the use of the Internet as a platform for fully integrated expert systems has not yet been attempted. Given the popularity of the MicroLEIS website, there is a clear need for a fully integrated webbased land evaluation expert system.

\subsection{SUMMARY}

Land suitability analysis involves the evaluation of land properties against land use requirements to determine if a parcel of land is suitable for a particular use. Because the number of properties (also called land characteristics or qualities) is potentially large and geographical in nature, spatial techniques and technologies such as Boolean overlay, multi-criteria decision making and expert systems have become fundamental tools to support the decision-making process.

Although Boolean overlay has the advantage of being simple to understand and easy to implement with standard GIS software, MCDM has established itself as a technique for supporting complex decisions involving numerous spatial factors with varying levels of importance. A major advantage of MCDM for developing SDSS is its ability to generate alternatives by modifying the importance levels (i.e. weights) of individual criteria. MCDM also supports fuzzy classification, which is a less discrete or 'hard' decision strategy.

A convenient approach to land suitability analysis is to use expert systems. They rely on expert knowledge stored in a database (or knowledge base) in the form of land requirement rules. Boolean or fuzzy rules are defined according to known land use requirements or they can be obtained from land use experts. The rules are applied to a database of land units using an established protocol (i.e. inference engine) to calculate suitability values.

The main advantage of the expert system approach is the way in which the rules are separated from the data. Very little data preparation is needed for expert systems because measurement standardization is inherent in the rules. This enables the use of standardized rules that are independent of the geographical area in which the land suitability assessment is carried out. In addition, it facilitates system automation and implementation, especially in a multi-user, readonly environment such as the Web where data-editing and creation capabilities are limited. Expert systems are also compatible with database management systems, web scripting and web mapping services for carrying out suitability analysis. The next chapter explores the possibilities of developing a Web-based land evaluation system. 


\section{CHAPTER 3: WEB MAPPING TECHNOLOGY}

Since its inception in 1989 the World Wide Web (WWW) or Web, has undergone many changes to the technologies on which it relies. In spite of forecasts to the contrary in the 1990s, hardware and infrastructural improvements have significantly speeded data transfer via the Internet. In terms of software, web browsers have become increasingly sophisticated and now have capabilities for rendering almost any type of information. These technological improvements have demonstrably impacted the way geographical information is communicated by making the visualization of spatial data on the Internet a reality. Web mapping tools, such as Google Maps (Google 2005), MapMachine (National Geographic Society 2005) and StreetMap (MWEB 2005), enable anyone with access to a computer and the Internet to explore geographical data online and produce maps on demand. As a result, more people access geographical information through the Internet than via any other medium (Longley et al. 2002). It is this popularity of the Internet as vehicle for delivering spatial information that this research aims to exploit.

In order to develop a Web-based land evaluation system, a thorough understanding of the available technology is required. This chapter focuses on the current Web technologies and concepts related to Internet mapping applications. This is done by first examining each of the major web components, followed by a description of the type of web maps published on the Internet. The chapter concludes with an overview of existing web mapping services.

\subsection{WEB APPLICATIONS}

A web application is a web browser-based application that is accessed via a computer network such as the Internet or an intranet. Essentially, a web application is a website that provides a specific function such as Internet banking, online stores, electronic discussion groups and web mail (e.g. Microsoft Hotmail and Google Gmail).

The main benefit of a web application is that no software other than a standard web browser is required to use the application. This gives developers the ability to update and maintain applications without the need to distribute (and redistribute) software to potentially millions of clients. This client-server architecture is not a novel idea as it is the basis on which mainframes and minicomputers have been functioning since the early 1970s. More recently, the dumb terminals used by these systems have given way to multiple-task personal computers running web browsers.

In addition to cost savings effected by the distribution and maintenance of web applications, users also benefit because only standard web browser software is needed to run such 
applications. Web browser software is not only free, but it also supports most platforms (i.e. Windows, Linux, Macintosh etc.) making web applications platform-independent. Users also do not need powerful computers as most of the processing takes place on the web server. Another significant benefit of web applications is that users require little additional skills or training to use them as the interface consists of standard web pages and components such as text, images, form fields and buttons, with which most users are familiar. This means that users are more likely to adopt and use web applications than other forms of implementations.

The main limitation of web applications is that users need an Internet connection to use them. The speed of the connection (i.e. bandwidth) is also a limiting factor because complex graphics and large volumes of information are time-consuming and costly items to download, making such systems sluggish and unresponsive. Although many of these limitations can be overcome with high-speed (i.e. broadband) Internet access, this technology is unlikely to become readily available to all South Africans.

While the familiarity of web interfaces improves user acceptance and adoption, the interfaces also limit the flexibility of web applications. The graphic capability of web applications is especially restrictive. Consequently, web mapping applications are difficult to implement and they are usually limited to viewing and manipulating existing drawings or maps. There are, however, several web applications, such as Google Maps (Google 2005), MapMachine (National Geographic Society 2005), AlertNet (Reuters Foundation 2005) and StreetMap (MWEB 2005), that allow users to view, edit and create maps online.

\subsection{WEB COMPONENTS}

Information on the Internet is mainly stored as web pages, which can be downloaded and viewed by Internet users. The web pages are physically stored on computers, or web servers, permanently connected to the Internet. Specialized web browser software is used to access web pages. To initiate a request for a web page, the user enters a uniform resource locator (URL) into the web browser. A URL is simply an easily recognizable representation of the web server's Internet protocol (IP) address, which is stored in a distributed Internet database. Once the browser has looked up the IP address, the browser sends a hypertext transfer protocol (HTTP) request to the server to retrieve the required page. The page is then sent back to the web browser to be rendered along with any files referenced by it. Six interrelated web components crucial to its application for web mapping are examined in the following sections. 


\subsubsection{Web browsers}

Much of the process involved in retrieving a web page is handled by web browser software mostly hidden from the user. Popular browsers such as Microsoft Internet Explorer, Mozilla Firefox and Opera can furnish many types of information including text, graphics, audio, interactive multimedia, and applets (i.e. small software components).

Although the basic file format for a web page is HTML (hypertext markup language), most browsers natively support a variety of additional image file formats, such as JPEG, PNG and GIF, and they can be extended to support more through the use of plug-ins. The combination of HTTP content type and URL protocol specification allows web page designers to embed images, animations, video, sound, and streaming media into a web page, or to make them accessible through the web page. The way in which text, images and other media are presented to the web user is defined by a markup language.

\subsubsection{Markup languages}

A markup language combines text and information about how text should be structured and presented. The best-known markup language is HTML, a derivative of standard generalized markup language (SGML) developed in the 1960s. The first version of HTML (called HTML Tags) was introduced by (Berners-Lee 1991) to describe the structure of a web page and has since been revised and extended several times to its current version 4.0.

The structure of web pages is defined through the use of a hierarchy of parent- and child-HTML elements (or tags) that are interpreted by the web browser. An example of a HTML document is shown in Figure 3-1.

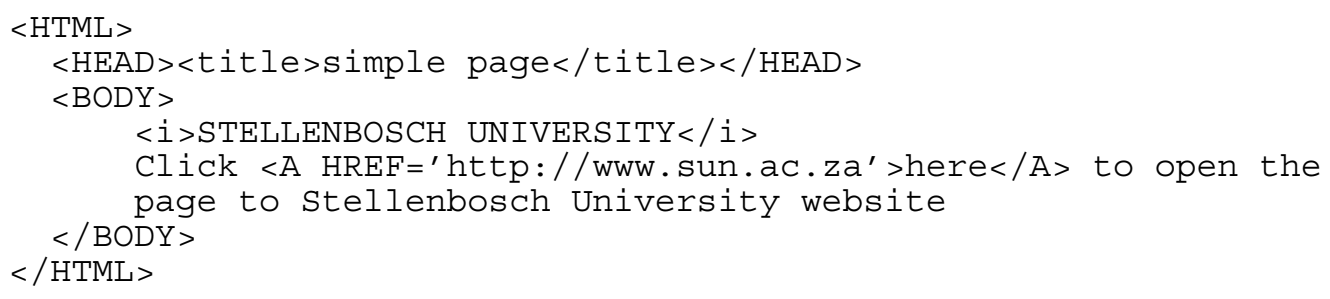

Figure 3-1 Example of a HTML document

In the first line the HTML label is used to indicate that the document is in HTML format. The HTML element is called the root element as it can have no parent elements. All element labels must be enclosed in corner brackets (i.e. $<$ and $>$ ) and terminated by a corresponding end label. End labels are identified by the forward slash character '/' before the label. For instance, the 
HTML label needs a corresponding /HTML label to end its influence, as shown in the last line of the document.

An HTML document usually consists of a heading and a body section. The heading section contains meta-data and is identified with the HEAD element. The heading can have a number of child elements, including the title of the document as specified using the TITLE label. The body section is defined using the BODY label and includes the visible contents of the web page. HTML elements not only define the structure of the page, but can also be used to describe how the content will be presented. For instance, the I element can be used to display text content as italic as implemented in line four of the example. Elements can also be used to link parts of a web page to other web pages. This is done through the A element, which specifies a hyperlink to another web page that will open when selected. The A element is an example of an element that requires attributes to influence its behaviour. In this example, the HREF attribute is used to specify the address of the hyperlink.

Although there are currently 91 HTML elements from which a web developer can choose, there is a constant demand for more elements with more functionality (W3C 2008). This demand has led to the development of extensible markup language (XML), a general-purpose markup language through which additional elements can be created as needed (W3C 2006). Each element is defined in a document-type definition which is interpreted by the browser. It is important to note that XML is not a replacement of HTML, but merely an extension.

Figure 3-2 illustrates how XML can be used to store the contents of an email. The first line declares that the document is in XML format (version 1.0) using a Latin/West European character set (ISO-8859-1). The root element EMAIL is specified in the second line and defines the type of object that the XML document is describing. The next three lines specify three child elements, namely ADDRESS, SUBJECT and BODY. Each child element contains the relevant data and is terminated using syntax similar to HTML. The end of the document is defined by the EMAIL element.

$<$ ?xml version="1.๑" encoding="IS0-8859-1"?>

$<$ EMAIL>

$<$ ADDRESS >avn@sun.ac . za</ADDRESS $>$

$<$ SUBJECT $>$ XML example</SUBJECT $>$

$<$ BODY $>$ This is an example of an e-mail stored in $\mathrm{XML}</ \mathrm{BODY}>$

$</$ EMAIL $>$

Figure 3-2 Example of an email stored as XML

Although the examples above make XML and HTML seem similar, the two markup languages have two different purposes. Where HTML instructs web browsers how content should be 
interpreted, XML is used for data storage and transfer. XML syntax is more restrictive than HTML and is therefore more easily parsed by web browsers.

With the continuous elaboration of HTML to include progressively more functionality, the computer memory and processing capabilities needed to render web pages have also increased. This is problematic for small devices such as cellphones and palmtops that have limited processing capabilities. As a result, XML was used to develop an additional markup language called extensible hypertext markup language (XHTML). Essentially, XHTML is a more efficient version of HTML due to the formatting restrictions placed on its structure and it is widely used for small devices (W3C 2002).

Web pages can be created using a combination of HTML, XML and XHTML code. Once the code has been parsed and translated by the web browser, it is displayed as text and images. Unfortunately, web content created by markup languages is static, which has led to the development of client-side scripting.

\subsubsection{Client-side scripting}

Client-side scripting refers to a class of programs executed by a web browser to alter the display and behaviour of static HTML web-page content. The term 'client-side' indicates that the operations are carried out on the client's (user's) computer or device, enabling web pages to react faster to users' actions. Client-side scripting is often used to make web pages more interactive and dynamic. Because of this effect, web pages that use a combination of HTML and client-side scripting are said to be created using dynamic hypertext markup language (DHTML) (W3Schools 2008a).

Another important function of client-side scripting is to improve the robustness of websites and applications. With scripting, users can be limited to perform only certain actions, depending on the current status of the application (Köbben 2001). For instance, client-side scripting may be used to restrict users to enter only numbers into a form field. Client-side scripting can also be used to guide users through interactive dialogs such as warnings and choice menus, thereby limiting errors and improving system usability (Canter 2004).

The most popular client-side scripting language is JavaScript (Mozilla Foundation 2008). This language, introduced in 1995 by Netscape, can be used to manipulate web page objects such as windows, documents, links and forms to perform a wide range of tasks. JavaScript code (see Figure 3-3) can either be incorporated in a web page or it can be called from a separate file. The advantage of keeping code separate from the web page is that the same code can be reused by different web pages, thereby limiting duplication and coding time. 


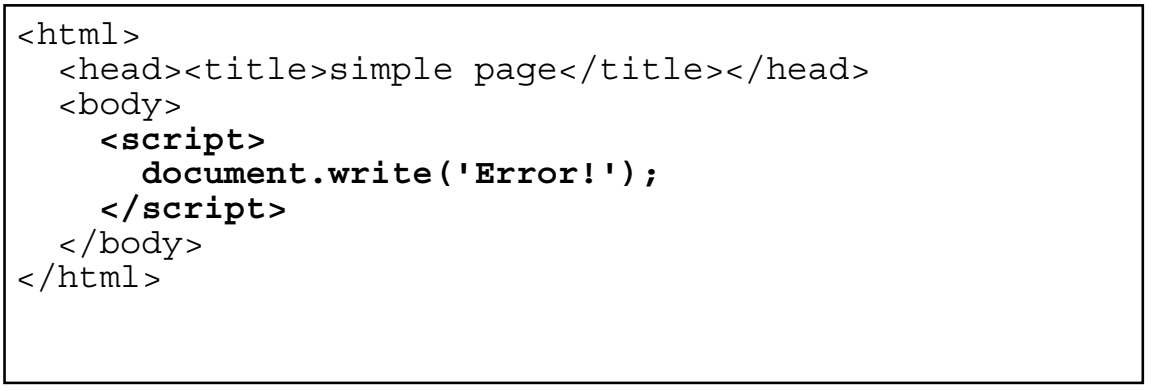

Figure 3-3 Example of JavaScript code that displays an error message when the web page is opened.

Whether JavaScript code is imbedded into a web page or loaded from a separate file, all the code that is referenced by the web page must be downloaded from the server before it can be executed. The more complex applications become, the more code needs to be downloaded, which can slow down the overall performance of a website. In addition, JavaScript can only perform operations on information that has already been downloaded from the web server. This not only limits its capabilities, but also poses security risks as sensitive information or code, such as financial records and passwords, can be viewed by any Internet user. Client-side scripting should therefore be limited to operations concerning the user interface and should not be used to retrieve sensitive information from the web server. Instead, for secure, dynamic information retrieval, server-side scripting should be used.

More advanced client-side functionality can be implemented using Java, an object-orientated programming language that is platform independent (Sun Microsystems 2008). However, in contrast to JavaScript which is inherently supported by most web browsers, applications developed in Java require special interpreters that need to be installed on the client. Similarly, web browser plug-ins can also be installed on clients to extend the functionality of existing web browsers (Köbben 2001).

\subsubsection{Server-side scripting}

The development of web pages using HTML, XML and JavaScript can be very costly and timeconsuming processes, especially for websites with large volumes of information that must be updated regularly. Using server-side scripting, web pages can be created automatically on request. In essence, the main function of server-side scripting is to instantly generate HTML, $\mathrm{XML}$ and JavaScript code to meet the requirements of a specific user. For instance, when an Internet banking user requests to view a financial statement, the web server generates unique code to display the relevant information. A new web page, based on the information supplied by the user, is created and once the page has been downloaded, it is removed from the server.

A major advantage of server-side scripting over client-side scripting is that operations are carried out on the server before any information is sent to the client. Because the scripts and operations 
are hidden from the web user, less data needs to be sent over the Internet. This improves website responsiveness and security. The downside of server-side scripting is that the server carries more load due to the additional processing required to execute the scripts and retrieve the necessary information. Fortunately, technological advances in computer processing alleviate much of the demand for additional processing power.

Fundamentally, any programming language can be used for server-side scripting, although several languages have been specifically developed for this purpose. Of these, PHP (PHP hypertext preprocessor) (35\%) and ASP (active server pages) (21\%) are the most popular (Nexen 2007). Although PHP can be run on any web server, it is most often used for Linux implementations, while ASP, developed by Microsoft, runs only on Windows-based web servers.

Other server-side solutions include common gateway interfaces (CGI) and application programming interfaces (API). While CGI scripts are used to develop interfaces between existing software (such as a GIS package) and web server software, API are server-side programs (often written in Java) to extend the functionality of web servers (Jiang 2003).

\subsubsection{Web servers}

A web server is a combination of hardware and software. As for hardware, essentially any computer can be used as a web server. However, most servers are dedicated computers with special hardware configurations to handle requests from a large number of users. Web servers are generally equipped with powerful processors and large volumes of computer memory. Most server hardware is currently supplied by IBM (31\%) and Hewlett-Packard (28\%), while DELL $(12 \%)$ is becoming a strong contender with a market share growing by more than six per cent annually (Modine 2007).

A number of web server software programs are available. Although more than half of all web servers run Apache software, current trends indicate that Apache will be replaced in 2008 by Microsoft's Internet Information Services (IIS) software as the most popular web server software (Netcraft 2007).

Many web servers include a database component to manage the large volumes of data that some web applications require. Information is extracted from the database and dynamically converted to web pages using server-side scripting. Databases can either be hosted directly on the web server or they can be stored on a dedicated database server to relieve the load on the web server. The configuration will greatly depend on the size of the database and the database management system being used. 


\subsubsection{Relational database management systems}

A database management system (DBMS) is a complex set of software programs specifically designed to control the organization, storage and retrieval of data from a database. Although several types of DBMS exist, relational DBMS (RDBMS) are the most popular. The relational database model, first proposed by Codd (1970), uses predicate logic and set theory to represent all data as mathematical relations.

The relational model consists of three data components: structure, manipulation, and integrity. Data structure refers to the organization of data, while data manipulation signifies the types of operations users can perform on the structure. The set of 'business' rules governing how data values behave on these operations, is known as data integrity (Fleming \& Von Halle 1989).

Relational data is organized into two-dimensional tables (also called relations or entities). Each table consists of a set of uniquely named columns (also called attributes or fields) and unnamed rows (also called tuples or records). To be relational, the data must be organized in the table so that each row is unique. Entries in columns must be single-valued and one kind, while the sequence of columns (left to right) and rows (top to bottom) is insignificant.

Data in tables can be manipulated by relational assignment. Although relational assignment is similar to variable assignments in computer programming, in relational databases the variable is a table and the assignment expression involves other tables. Eight operations, namely SELECT, PROJECT, PRODUCT, JOIN, UNION, INTERSECTION, DIFFERENCE and DIVISION are available for relational assignments (Fleming \& Von Halle 1989).

Data integrity, the third component of the relational model, is governed by rules that constrain permissible values in the table columns and the actions that should be taken to remove records. For example, the entity integrity rule states that no null values (i.e. empty or zero) are allowed in primary keys, that is the column or set of columns that uniquely identify each row. Another important rule to ensure data integrity is the referential integrity rule which addresses the integrity of foreign keys. A foreign key is a column or set of columns functioning as a primary key in another table. The rule states that the values in a foreign key must be either null or must have values matching the values in its corresponding primary key. There are many other rules meant to deal with the integrity of all columns, including primary and foreign keys. These socalled 'domain integrity rules' restrict column entries to values that correspond to each column's domain. A domain refers to a logical pool of permissible data types (e.g. text, number), lengths and ranges as well as settings such as default values, uniqueness, and nullability. Although the relational model does not dictate how data integrity is implemented, integrity is a logical and 
integral part of any relational database and should be defined and endorsed without involving the user in the technical implementation (Fleming \& Von Halle 1989).

Users and database developers usually interact with databases through a standard database language called structured query language (SQL), which has two main functions. First, it can be used to manipulate the data in a database through the use of SQL relational operators such as SELECT, INSERT, UPDATE, DELETE, JOIN and UNION. An example of a SELECT statement combined with a JOIN is shown in Figure 3-4.

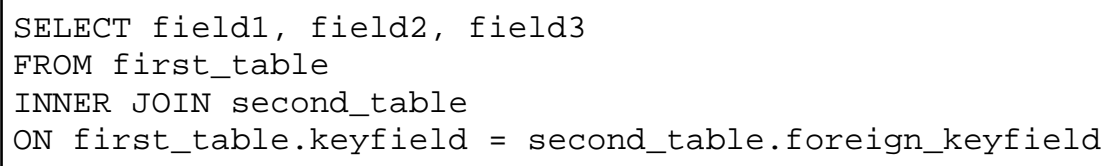

Figure 3-4 A SQL statement using the SELECT and JOIN operators

The second function of SQL is to alter the structure of an existing database or create an entirely new database using operators such as CREATE, ALTER and DROP. Although some database software includes additional operators, most relational database software can be accessed and manipulated using the same basic SQL statements (Fleming \& Von Halle 1989).

A number of RDBMS software packages is available. According to Pettey (2007), Oracle currently produces the most popular database software which commands $47 \%$ of the market share, while other popular DBMS developers include IBM (21\%) and Microsoft (17\%). Oracle and IBM's software offerings are aimed at the enterprise level market, while Microsoft also caters for the small business and home office applications with their Microsoft Access software. Owing to its portable file structure, Microsoft Access is also widely used for web applications and it is the database of choice for rapid application development because it is so easy to set up and manipulate. Due to its user-friendly interface and compatibility with other Microsoft products, many developers prefer to use Microsoft Access to design and implement prototype databases. Once the prototype is stable in terms of its structure, or when the size of the database nears the software's limitations, it is usually replaced by a more robust RDBMS such as Oracle or SQL Server. The latter is Microsoft's enterprise database solution (Microsoft 2007).

The choice of a RDBMS largely depends on the type, size and complexity of the web application for which it will be used. However, it is essential that the server-side scripting language and the RDBMS are compatible. Fortunately, many operating systems or third-party developers offer standard open database connection (ODBC) interfaces to translate requests form web applications into a format that DBMS can interpret. 
The web components described above illustrate the complexity of web applications. While RDBMS and server-side scripting are used to dynamically generate web pages, client-side scripting, HTML, images and other media are used to create dynamic web pages. An additional level of complexity is added by web applications that produce dynamic web maps.

\subsection{WEB MAPS}

Web maps refer to all the types of maps distributed via the Internet. Although there are various types of web maps, nine types, distinguished by their characteristics, are overviewed in the next section, followed by an exposition of their formats.

\subsubsection{Characteristics of web maps}

Web maps can be characterised as being either static or dynamic. In addition, each of these main web map types can be further classified as view only and interactive web maps (Figure 3-5). These, as well as some additional characteristics of web maps (distributed, animated, real-time, personalized, reusable, and collaborative), are considered below.

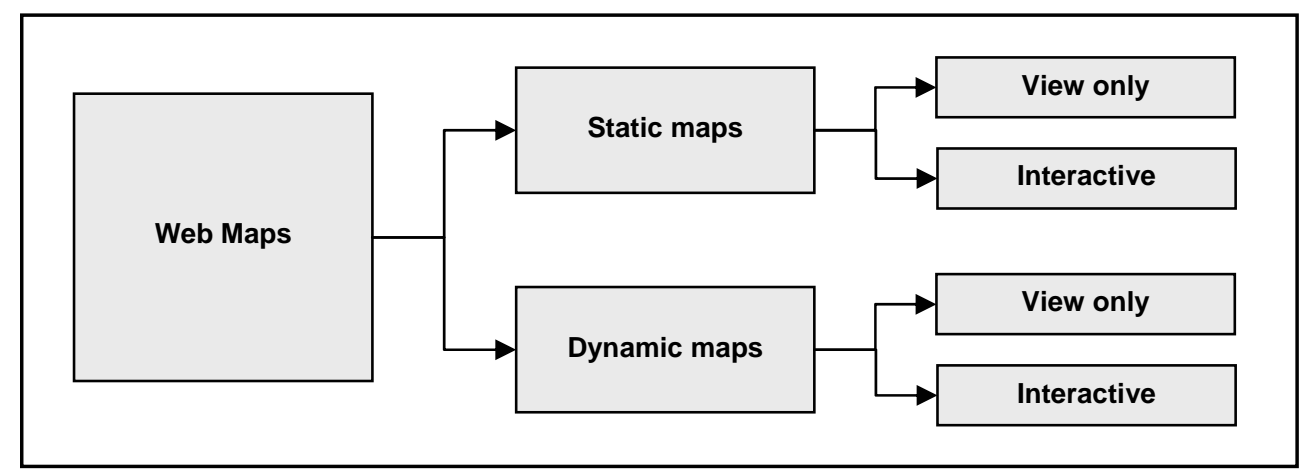

Figure 3-5 Classification of web maps

Adapted from Kraak (2001)

\subsubsection{Static vs. dynamic}

Static web maps are similar to paper maps in that they are created once and are infrequently updated (Kraak 2001). Although many static maps are created specifically for Internet distribution, some static maps are simply digital (scanned) versions of hard-copy paper maps. Such maps are not always suitable for Internet use because the high resolution needed to accurately represent the quality of the original printed map often results in file sizes being too large to download (Peterson 2003). Consequently, some mapping applications allow users to select sections of maps for downloading. In addition, client-side and server-side scripting is used to let users change (i.e. pan) the extent and position of these sections interactively, or enlarge and reduce (i.e. zoom) the map scale as needed. Usually, such dynamic web maps are created each time it is downloaded. The map is therefore not stored on the server, but is dynamically 
generated from a database or GIS on request. Specialized server-side programs, called web map service (WMS) software, are required to convert the source data into map format.

\subsubsection{Interactive vs. view only}

Client-side scripting can be employed to enhance view only or non-interactive web maps by providing functions like hyperlinking and active areas through which users can open other spatially related web pages or multimedia by clicking on different places on a map (Taylor 2005). Although static maps are frequently used as bases for producing these so-called 'clickable' maps, many interactive maps are created dynamically.

\subsubsection{Distributed}

Some dynamic web maps are created from distributed data sources located on different WMS. Such maps, called distributed maps, are requested through a standardized protocol understood by all the relevant WMS. Maps are delivered along with service-level metadata and map feature attributes (Tsou 2003). A list of available WMS are listed on wms-sites.com.

\subsubsection{Animated}

Although dynamic or even static maps can be made to appear animated (i.e. with animated symbols or panning) the term animated web map refers to a map that illustrates spatial change over time using animation (Peterson 2003). A good example of such a map is a weather map showing the movement of a weather system. Animated web maps are produced by displaying a sequence of static maps to give the appearance of movement (Cartwright 2003).

\subsubsection{Reusable}

Due to the cost of producing and serving web maps, some companies sell maps to web developers who cannot produce maps themselves. For instance, Google Maps allows other websites to use (or reuse) maps that are dynamically generated on Google's servers. Although Google maps appear to be part of the website in which they are displayed, the actual map content is downloaded from a Google Maps server each time the particular web page is accessed.

\subsubsection{Real-time}

A major advantage of the Web over other means of map publication is that distribution can be nearly instantaneous. This enables the mapping of phenomena in real-time. An example of such an application is satellite tracking of vehicles which permits owners to view the position of their vehicles at any given moment (Altech Netstar 2008). Real-time maps can also provide functionality such as location based services (LBS) (Gartner 2005). 


\subsubsection{Personalized}

Some web applications give users the functionality to produce personalized maps. Examples are occasion maps (e.g. location of a function or meeting) or maps showing directions to a particular location (e.g. conference venue, office, shop, hotel). With such applications, users can specify the map's extent, scale and visible features (i.e. layers) as well as colour schemes and unique symbolizations.

\subsubsection{Collaborative}

A collaborative web map is a map created and edited by various users (Caquard 2003). An example of a collaborative mapping application is WikiMapia, an implementation of reusable Google Maps that allows users to add information to any location on earth. Although the technology is still immature and complex, it has much potential, especially for data collection and spatial applications in which participation is required.

The above discussion of web map characteristics is not exhaustive, but it provides a good idea of the types of web maps now in use. Additional types of maps and features are expected to appear as web mapping develops and new uses for web maps are found. Although different types of technology are used to create and distribute web maps, the format of maps on the Web is restricted by the formats recognized by web browsers. The next section is a synopsis of the types of formats used for web maps.

\subsubsection{Formats of web maps}

Like GIS data, web map formats are of two kinds - raster and vector - each considered separately below.

\subsubsection{Raster}

Raster files, or images, are the most common data format for web maps. A raster is a twodimensional array of grid cells or pixels (short for 'picture elements') with each cell representing a colour-intensity value. By combining three rasters representing red, green and blue (RGB) respectively, a full-colour image can be formed. The number of distinct colours that can be rendered by combining three rasters (colour bands) depends on the number of intensity values allowed in each grid cell. For instance, if each grid cell can store 256 values ( 8 bits), then a RGB image can store a total of $256 \times 256 \times 256=16777216$ (or $2^{24}$ ) distinct colours. Such images are called 24-bit images because they can store $8+8+8=24$ bits of data (colours).

Most web maps are stored as either graphics interchange format (GIF) or joint photographic experts group (JPEG) images (Peterson 2003). The major difference between GIF and JPEG 
images is the number of colours that each can represent. Where 8-bit GIF files can only display 256 distinct colours, JPEG images can represent up to $2^{24}$ colours due to its 24-bit capability. In general, images require large disk storage space and are slow to download via the Internet. To limit file size, GIF uses run length encoding (RLE), a popular compression technique that can be reversed (i.e. uncompressed) without any loss in quality. RLE compression is only efficient for images that include large homogeneous areas. For photographs containing a high level of colour variability, JPEG format is more suitable.

To sufficiently compress the additional colour information stored in JPEG images, a specifically designed compression technique is used that generalizes colour variation. This so-called 'lossy' compression technique is non-reversible, meaning that the quality of the original image is lost once compression has been carried out. However, the level of compression can be limited to restrict the loss of quality to a degree that it is unnoticeable in most applications.

Although JPEG and GIF are the most popular raster formats on the Web, a third format called portable network graphics (PNG) is rapidly gaining popularity. PNG was designed in 1996 specifically for transferring images over the Internet and is similar to GIF. The major advantage of PNG over GIF is that it has bit depth of 24 , which enables it to display up to $2^{24}$ colours. PNG therefore combines the advantages of 'lossless' GIF and the colour range of JPEG. As with GIF, PNG format is not suitable for images with a high level of colour variability.

Raster-based web maps are popular because they are compatible with computer data structures (e.g. arrays) and digital monitors. Web maps in this format can also be rendered by web browsers without the need for any additional software. The main drawback of raster-based web maps is the inflexibility of the scale at which they can be displayed. Images are usually created for the resolution at which they will be viewed (e.g. 96dpi for the Microsoft Windows operating system) and cannot be enlarged without loss of detail. This limitation can be overcome by using vectorbased graphics for creating web maps.

\subsubsection{Vector}

The vector data model stores spatial features as points, lines and polygons. Points are represented by individual coordinate pairs referenced to a common coordinate system. Lines are sequences of points that are connected, while polygons (areas) are closed lines (i.e. their starting and ending points coincide).

Three vector formats, namely portable document format (PDF), scalable vector graphics (SVG), and shock wave flash (SWF), are currently used for web maps (Peterson 2003). Each has different capabilities. Due to their portability and compatibility with desktop publishing, PDF is 
very popular for the distribution of static maps. SWF (also called Flash) is a powerful medium for producing interactive, animated web maps. Because maps in PDF and Flash format need to be downloaded fully before they can be viewed, they are not suitable for dynamic, distributed, personalized or collaborative web mapping. The only vector format that can be used to create web maps with these characteristics is SVG, an open standard for vector graphics on the Web. SVG is based on XML and is therefore highly compatible with HTML and related formats. Unfortunately, not all web browsers are capable of rendering SVG data and special software (plug-ins) is required. The lack of web browser support for the SVG format has limited its adoption by users and developers. However, SVG is a relatively new format and it is expected that all web browsers will support the format in the near future.

\subsection{WEB MAP SERVICES}

As discussed in Section 3.3.1.1, the function of a web map service (WMS) is to produce and serve dynamic web maps. Requests for dynamic maps are usually received in a standard WMS format, as specified by the Open Geospatial Consortium (OGC) (De la Beaujardiere 2004), and interpreted by the software to extract the necessary data from a GIS database. Based on the user's requirements, the spatial data is then converted to a map and served through a web server as an image or a set of vector features viewable with a web browser.

A WMS comprises three functional components: (1) data storage and retrieval; (2) map production; and (3) map distribution. Each of these components is described in the following sections.

\subsubsection{Data storage and retrieval}

Web applications allow users to download data, but rarely allow them to upload data. This limitation mainly exists to prevent users from uploading any harmful content such as computer viruses and it ensures that hackers cannot attack and corrupt systems. Allowing users to upload data also causes logistical problems for storage space and access rights.

Internet mapping has additional limitations regarding users uploading data because access to the map server software - running on the web server - is required to set up and design the maps that will be served. For security reasons, access to this software is usually limited to an administrator because anyone else with access could edit or even delete the maps being served. To reduce the security risk, a collection of spatial data sets is available for viewing and users needing specific data for their analyses can request it to be manually loaded by the system administrator. 
The GIS data used by a WMS can be stored in several formats. Popular formats for vector data include SVG and shape file format, while tagged image file format (TIFF) or JPEG 2000 format are often used for raster data. Storing large GIS data sets in these file structures is sometimes inefficient because the entire data set needs to be loaded onto the server's memory. Although this limitation can be overcome by using spatial indexing (i.e. divide the data into smaller spatial units and only load the necessary areas into memory), many WMS applications rely on RDBMS (see Section 3.2.6) for the storage and retrieval of spatial data. RDBMS not only improves efficiency, but also protects data integrity by managing events like simultaneous requests from multiple users. RDBMS has other useful data management functionality such as regular backups and versioning (the ability to centrally monitor changes and to roll back or undo to a previous version).

Unfortunately, standard RDBMS are not suitable for storing geographical features such as lines and polygons requiring variable length records (a line can theoretically consist of an unlimited number of coordinate pairs). Because most RDBMS are designed to mainly store text strings and numbers, they are also not good repositories for raster data. Standard RDBMS can however be modified by adding additional software to manage the conversion of spatial data into non-spatial data structures. The need for this software has prompted several commercial RDBMS, such as Oracle and Informix, to offer spatial extensions which handle these conversions automatically (IBM 2007; Oracle 2007). ESRI has produced separate spatial data engine (SDE) software, called ArcSDE, which manages the storage of spatial data in Oracle, Informix, DB2 and Microsoft SQL Server databases, without the need for spatial extensions (ESRI 2007c).

Regarding hardware, the main requirement for the storage and retrieval of spatial data is harddrive space. WMS implementations such as Google Earth and the United States Geological Survey's (USGS) Earth Resources Observation and Science (EROS) data centre require enormous volumes of hard-drive space. Multiple, dedicated data servers are also needed to process the continuous requests for data. Other hardware requirements include tape drives for backing up data as well as network infrastructure to connect to the servers that are responsible for map production. 


\subsubsection{Map production}

The production of dynamic maps for Web distribution has special software and hardware requirements. WMS software can be categorized into open source (OS) and proprietary software. Whereas the intellectual property of proprietary software is owned by an individual or a commercial company, the intellectual property of OS software is relaxed or non-existent. This means that the source code of OS software is available to anyone and the software can be obtained and used for free. According to the Open Source Initiative (Open Source Initiative 2007: s.p.), OS is "a development method for software that harnesses the power of distributed peer review and transparency of process...[which promises]... better quality, higher reliability, more flexibility, lower cost, and an end to predatory vendor lock-in.”

Examples of OS WMS software are ALOV Map, GeoServer, GeoTools, MapIt!, MapServer and MapZoom. Of these, MapServer is currently the most popular with 49 active public WMS listed on wms-sites.com and more than one hundred implementations listed on the MapServer website (Lime 2006). MapServer, originally developed by the University of Minnesota in cooperation with the National Aeronautics and Space Administration (NASA) and the Minnesota Department of Natural Resources, is now maintained by a number of developers internationally. The software is extremely versatile regarding the server-side scripting (e.g. PHP, Python, Perl, Ruby, Java, and C\#) and the platforms (e.g. Windows, Linux, Mac OS X and Solaris) it supports. MapServer is also compatible with several RDBMS including Oracle Spatial, MySQL and PostGIS (Lime 2006).

Probably the largest MapServer implementation is the United Nations Environment Programme (UNEP) GEO Data Portal, an online database of more than 450 environmental variables. Other MapServer applications include travel maps (e.g. Komotini City Guide; Yosemite National Park Hiking Map; Winnipeg Restaurants), online atlases (e.g. Atlas of Eastern and Southeastern Europe; Atlas Amazonas; Atlas of Canada) and local authority implementations (Bayawan City Online Project Monitoring System; Naga City, Philippines; Minnesota Land Use and Land Cover Map).

Whereas OS WMS software are mostly stand-alone packages focused on serving web maps, proprietary WMS software packages are usually extensions of existing GIS. Examples are Autodesk's Mapguide, Intergraph's GeoMedia WebMap and ESRI's ArcIMS. Of these packages, ArcIMS is currently the most popular with 121 public WMS listed on wms-sites.com.

Due to its scalability and robustness, ArcIMS is regularly used for extensive applications such as National Geographic's MapMachine (nationalgeographic.com), the USGS EROS data centre 
(edc.usgs.gov), and Canada's Geoscience Data Repository (gdr.nrcan.gc.ca). ArcIMS is popular in South Africa, where it is used by the Department of Water Affairs and Forestry map services (dwaf.gov.za), the Council for Scientific and Industrial Research (CSIR) web map server (spatial.csir.co.za), and the South African National Department of Agriculture's Agricultural Geo-referenced Information System (agis.agric.za).

While the data storage and retrieval component of WMS is highly dependent on large volumes of hard-drive space, the conversion of GIS data into map format requires high processing capacity. To improve response times, multiple servers are often used to balance the processing load. However, the use of multiple map servers increases the complexity of the software needed to manage and perform the requests. To further reduce the load on map servers, separate servers are frequently used to distribute the maps to web users.

\subsubsection{Map distribution}

Once GIS data has been extracted and converted into a map format that is web browser compatible, the data is placed on a web server (Section 3.2.5) for distribution. The web server requires little disk space as the maps are stored only until a user sends a request for a different map. Web servers not only let users download maps but also handle requests for new maps. Requests are received as URL, which are passed to the map server software. Although each map server handles requests differently, many WMS have adopted Open Geospatial Consortium (OGC) specifications (Open Geospatial Consortium 2007) which enable users to send standardized requests to several WMS simultaneously to produce distributed maps (see Section 3.3.1.3).

\subsection{SUMMARY}

Web mapping applications are essentially web applications that serve, along with other online content, dynamic and interactive web maps. Web mapping applications are developed by using standard web components such as markup languages, client-side scripting, server-side scripting and relational database management systems along with special server-side software that produces maps on demand. The function of web mapping software is to accept requests for maps using a standardized protocol and to dynamically create maps from GIS data sets. The maps are converted into a format compatible with standard web browsers and are temporarily stored on a web server along with other web content (i.e. text, images and forms) for downloading. Succinctly, web mapping applications comprise a WMS and web pages in which the created maps are displayed. The next chapter explains how these components are incorporated into the 
Cape Land Use Expert System (CLUES) design to enable online suitability analysis functionality. 


\section{CHAPTER 4: $\quad$ REQUIREMENT ANALYSIS AND DESIGN OF CLUES}

As with most large software developments, the design of the Cape Land Use Expert System (CLUES) was preceded by a requirement analysis, which entails establishing and expressing the needs and constraints placed on a software product (Kotonya \& Sommerville 1998) while bearing in mind that a software requirement is a property the developed or adapted software must exhibit to solve a particular problem (Abran et al. 2004).

The requirements for CLUES outlined in this chapter were identified from the relevant literature by studying the functionality needed in order to perform land suitability analysis (see Chapter 2) and by examining the architectures and data used in similar existing systems. Good functionality, accessibility, user-friendliness and speed were singled out as the major factors contributing to the success of a land evaluation system. The discussion of the requirements is followed by a description of the system design which includes an overview of the implementation of the CLUES components.

\subsection{SYSTEM REQUIREMENTS}

The properties that CLUES should exhibit are divided into two categories. Essentially, a distinction is made between properties that are related to functional needs (i.e. what the system should do) and operational characteristics (i.e. how the system should do it). Because of the strong reliance of SDSS on spatial data, an additional category, data requirements, has been added.

\subsubsection{Functional needs}

The functional requirements of CLUES are directly related to the secondary aim of this research, which specifies that the system must enable users to perform spatial land suitability analysis for anywhere within the Western Cape. The literature review determined that, in order to perform land suitability analysis, users must be able to:

- collect and prepare data;

- identify the land uses to be evaluated;

- specify land use requirement rules;

- map land units;

- determine land properties;

- calculate (analyse) land suitability; 
- $\quad$ summarize and tabulate; and

- create suitability maps.

These main functions are similar to the land evaluation steps shown in Figure 2-1. They are however not exhaustive as additional functionality will be required to support these operations.

\subsubsection{Operational characteristics}

How the system's functionality is accessed, performed and presented are part of its operational characteristics. These characteristics are expounded in the following sections.

\subsubsection{Accessibility}

The research's aim stipulates that users must be able to access CLUES functionality via the Internet. This requirement has far-reaching logistical implications because the system must be able to handle multiple users concurrently. It must preferably do so without any user being aware of other users. This means that an individual working environment must be created for each user enabling them to input their own data, define their own land uses and set up their own land use requirement rules. Users must not only be allowed to perform individual suitability analyses; they must also be able to store suitability maps and parameters so that they can continue with a project at a later stage.

\subsubsection{Performance}

One of the main requirements of a SDSS is that it must facilitate scenario building. Users must be able to explore the data and interactively see the possible effects of different decisions. The system must be responsive to any changes in the criteria or rules and suitability maps must be created on demand without long delays.

Research about acceptable waiting times for web pages to open has provided inconsistent findings. According to Nah (2004), users are not willing to wait more than two seconds for web pages to download, while Dennis \& Taylor (2006) showed that seven seconds was considered to be acceptable. Selvidge (1999) reported that little difference in users' tolerance was observed between one-second waiting times and 20 -second waiting times, but that there is a marked difference between one-second and 30-second waiting times. Users' willingness to wait longer depends on the perceived complexity of the information requested (Bailey: s.d.). Longer waiting times are acceptable for content such as Internet searches, imagery and software downloads or when the requested information is of high importance (Dobbs 2004). 
Unfortunately, no research has yet been done on acceptable waiting times for maps. A useful guideline is that the response time of CLUES should match or improve upon those of GIS software. GIS users often have to wait long for maps to be drawn or for analyses to complete. For example, a simple overlay (union) operation of Western Cape farm boundaries $(\approx 50000$ polygons) and land uses ( $\approx 16000$ polygons) in ArcGIS takes more than one minute to complete. Because land suitability analyses often involve multiple overlay operations (one for each land property being considered), it is expected that a suitability evaluation will require processing times of several minutes. Such long delays might however dissuade users from exploring different scenarios. A possible solution to reducing delays might be to limit the extent of individual analyses.

To encourage scenario building, a system design goal of a maximum delay of one minute was set for a suitability analysis to be completed using CLUES. Response times for non-spatial functions, such as setting up parameters and rules, must preferably be less than seven seconds. To improve response times, provision of unnecessary information and imagery should be limited. The graphical user interface (GUI) must therefore be simple and functional, rather than elaborate.

\subsubsection{Presentation}

Because the system will be developed to allow access through the Internet, the interface through which the user will control the system will be a website. Using a website as interface can improve usability as most users are familiar with web pages and their components (i.e. text, images, forms and buttons).

To ensure that the CLUES website is intuitive, Nielsen's (1994) guidelines for user interface design will be followed, namely to:

- provide feedback to users in order to keep them informed about what the system is doing;

- use language, phrases and concepts that are familiar to the user;

- control user freedom in order to prevent users from choosing inappropriate options;

- allow users an 'emergency exit' so that they can return to a previous state, especially if the user has made an error;

- be consistent in the words, situations, or actions used;

- prevent errors by presenting users with confirmation dialogs;

- minimize the user's memory load by making objects, actions, and options visible; 
- introduce ways in which actions can be accelerated using shortcuts;

- not include irrelevant information in dialogs; and

- express error messages in plain language that precisely indicates the problem and constructively suggests a solution.

The CLUES user interface should not only conform to user-interface guidelines, but should also follow web-design principles. The web-design guidelines document developed by U.S. Department of Health and Human Services (2006), titled Research-based web design \& usability guidelines, was identified as the most suitable source for the purposes of this study. This comprehensive document (216 pages) includes strategies regarding the design process, makes suggestions on how to optimize the user's experience, and provides practical guidance about page layout, navigation, scrolling, paging, headings, links, and text.

\subsubsection{Data requirements}

Land evaluations conducted by others were studied to discover existing data sets essential for inclusion into CLUES. A wide range of applications was considered. These include suitability involving agricultural, forestry, environmental and urban land uses. Most of the applications used climate, soil, terrain, infrastructure and land use data. After stipulating an operational scale, the value of each of these data types is discussed in the next five subsections.

\subsubsection{Operational scale}

CLUES must allow users to work at the largest possible scale. Although Lambrechts \& Ellis (s.d.) suggest that scales ranging from 1:2 000000 to 1: 120000 are appropriate for areas as large as the Western Cape (see Section 2.1.1), the application of CLUES for suitability analysis will be more valuable if it can be used for semi-detailed (i.e. 1:100 000 to 1:30 000) investigations. Because South Africa has standardized on a scale of 1:50 000 for its largest-scale topographical map series covering the Western Cape (CDSM 2007b), and because much relevant data is mapped at this scale, it was decided that CLUES must have the ability to carry out suitability analyses at a scale of 1:50 000. All the data collected for use in CLUES should therefore preferably be at a scale of 1:50 000 or larger.

\subsubsection{Climate data}

Climatic data such as rainfall, temperature, humidity, and solar radiation are used in most of the land evaluations consulted. Rainfall and temperature are considered to be the most important climatic parameters (Ceballos-Silva \& López-Blanco 2003b; De la Rosa et al. 2004). Rainfall is especially significant for suitability evaluations concerning vegetated land uses because water is 
the most important factor in plant development. Water is essential for the maintenance of physiological and chemical processes in plants and acts as an exchanger of energy and a carrier of nutrients (Schulze 1997).

Rainfall is an essential factor for agricultural land uses, especially for determining the suitability of land for the production of specific crops (Ceballos-Silva \& López-Blanco 2003a). Rainfall also influences the distribution and occurrence of natural vegetation types, which in turn relate to the geography of ecosystems and fauna (Du Toit et al. 2002; Mucina \& Rutherford 2006). Most evaluations employ information about the total amount of rainfall per year as well as rainfall averages per month.

Temperature is frequently used as an index of the energy status in the environment and it affects all forms of life. Because of its influence on human comfort, temperature determines our demand for energy and therefore our dependence on resources (Schulze 1997). Temperature also strongly affects animal behaviour and responses, such as hibernation and migration (Walther et al. 2007), and it has a significant effect on community distribution and size (Botes et al. 2006). The occurrence of natural vegetation is strongly related to temperature as all plants have upper and lower temperature limits above or below which their growth development processes cease. Crops have different optimum temperature requirements for development processes such as photosynthesis, respiration and flowering. For instance, the optimum average temperature range for wheat is $25^{\circ} \mathrm{C}$ to $31^{\circ} \mathrm{C}$, while sorghum needs temperatures between $31^{\circ} \mathrm{C}$ and $37^{\circ} \mathrm{C}$ (Schulze 1997).

Three additional temperature-based variables were found to be popular and were considered fundamental to land evaluation. The first, heat units (also called growing degree days), is a heuristic index that is frequently used in phenology to predict crop maturity or bloom dates (Schulze 1997). Consequently, heat units are often used to identify areas suitable for crop production (Carey 2005). A second indicator often used in agricultural applications is chill units (also called positive chill units or chill hours). Chill units are defined as the accumulative number of hours that plants are exposed to temperatures ranging from $2.4^{\circ} \mathrm{C}$ to $9.1^{\circ} \mathrm{C}$ during winter. Chill units is an important factor in land evaluation as most deciduous plants require a minimum number of chill units to satisfy dormancy, to stimulate growth, develop leaves, flower and set fruit (Reiger 2006; Schulze 1997). Because too low temperatures can be damaging to plants, especially during the growing season, frost data was introduced as a third temperaturebased variable. Frost data is often used in land evaluations to identify areas that should be avoided for the production of particularly perennial crops. 
Frost, chill units, heat units, mean annual temperature, as well as monthly mean, minimum and maximum temperatures were included in most of the suitability analyses consulted and were consequently established as indispensable climatic data sets for CLUES.

Climatic data is obtained from long-term observations taken at weather stations and because weather stations are relatively sparsely situated, especially so in mountainous regions or areas with low population densities, climatic parameters at any given location are usually determined by interpolating values from the nearest weather station or stations (Ceballos-Silva \& LópezBlanco 2003b). Owing to the effects of topography on climate (in particular orographic precipitation and cooling), more realistic values are obtained when elevation is considered in interpolation processes (Joubert 2007).

\subsubsection{Soil characteristics}

Soil information is especially important in land suitability analyses that are concerned with vegetated land uses as most plants require soil for support (anchorage), water, oxygen and nutrients. Most plants also prefer soils with specific characteristics. In addition, soil data is significant for urban land uses, as sandy soils are more suitable as foundations for roads and buildings than clayey soils (Brown 2003).

Soils are formed through the combined effect of physical, chemical, biological and anthropogenic processes on the parent material. Parent materials consist of geological materials that have undergone some degree of physical or chemical weathering. The result is the formation of soil horizons or layers with distinctive colour and texture properties. These properties, together with the thickness and arrangement of soil horizons, are studied by soil scientists and classified into soil types. During a typical soil survey, classifications are conducted at several locations in the surveyed area and, together with terrain maps, used to produce a soil map. Terrain has a strong influence on soil formation as it not only influences erosion and drainage, but also determines where weathered materials are deposited (Schloms 2007, pers com; Soil Survey Division Staff 1993; Van Niekerk \& Schloms 2001).

Soil maps in their native form are of little use in automated land suitability analysis because soil types need to be interpreted by a soil scientist for a particular land use. Usually this involves creating groupings or ratings of soils according to their limitations, suitability and potentials for particular land uses (Soil Survey Division Staff 1993). To avoid this additional step, quantitative soil properties such as texture, depth and chemical characteristics are often extracted from soil type data and used in suitability calculations (Ceballos-Silva \& López-Blanco 2003b; Cools, De Pauw \& Deckers 2002; De la Rosa et al. 2004). 
Soil texture is one of the most important characteristics of soil as it directly relates to many other land properties such as available water for plants, permeability, infiltration, plant nutrients, erodability, tillage danger, and tillage strength requirements. While finer-textured soils are generally more fertile, contain more organic matter and retain moisture and nutrients better, too clayey soils are likely to be too difficult to manage and unstable between dry and wet periods. Sandy soils are more stable, but need frequent fertilization and good water management (Brown 2003; Lambrechts \& Ellis s.d.).

Soil texture describes the relative proportion of different grain sizes of mineral particles. Particles are grouped into soil separates (sand, silt and clay) according to their size. Sand particles are large $(0.1 \mathrm{~mm}$ to $2 \mathrm{~mm}$ in diameter), while silt and clay particles are small $(0.002 \mathrm{~mm}$ to $0.05 \mathrm{~mm}$ and less than $0.002 \mathrm{~mm}$ respectively). The percentage of each soil separate in a soil is used to classify soil texture into 12 major textural classes (Figure 4-1).

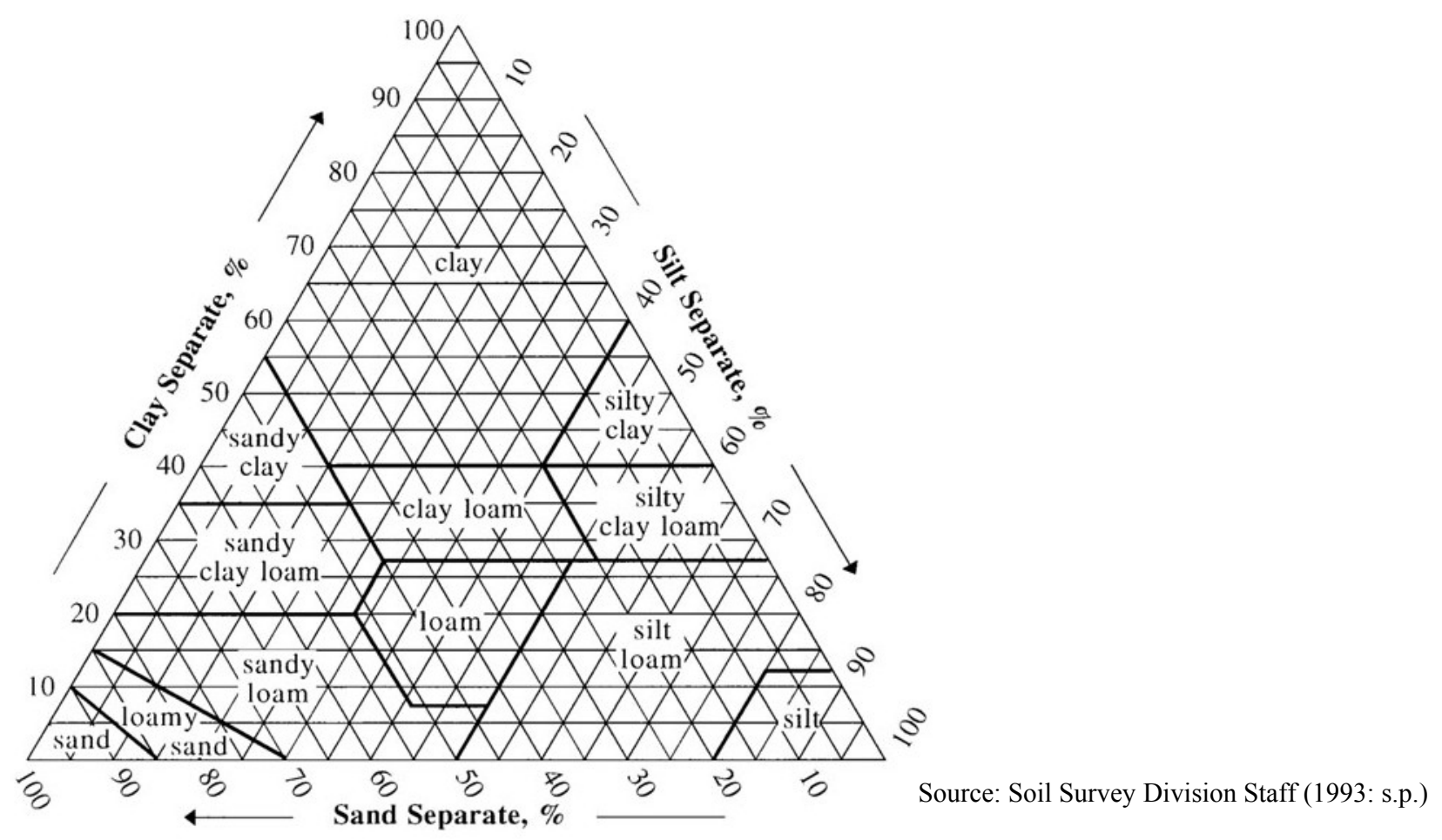

Figure 4-1 Soil texture triangle showing the twelve major textural classes and particle size scales

Although soil fertility can be partly ascribed to texture, soil reaction or $p H$ is another factor strongly affecting the nutrient availability in a soil. Soil $\mathrm{pH}$ is an indication of how alkaline or acid a soil is and ranges from 0 (very acidic) to 14 (very alkaline) and mainly depends on the type of parent material from which the soil was formed. Rainfall also affects $\mathrm{pH}$ as basic nutrients such as calcium and magnesium are often leached and replaced by more acidic elements such as aluminium and iron when water passes through soil. Soils in high-rainfall regions are therefore usually more acidic than those formed under arid conditions. Soil $\mathrm{pH}$ is important for land evaluations because many nutrients become soluble below a $\mathrm{pH}$ of 5 and they 
are available to plants only at these levels of acidity. Some plants need these nutrients in order to develop, while others have adapted to more alkaline soils. Too high acidity or alkalinity may become toxic (Soil Survey Division Staff 1993).

Another soil characteristic frequently used in land suitability analysis is effective soil depth or rooting depth (Cools, De Pauw \& Deckers 2002; Dendgiz, Bayramin \& Yüksel 2003). This refers to the depth, measured from the surface, at which root penetration would be strongly inhibited due to physical characteristics such as contact with bedrock, dense clay or permanent water, or due to contact with soils with extreme chemical properties (Soil Survey Division Staff 1993). Effective soil depth not only determines root growth, but also influences the waterretaining capacity of soils. Deep soils usually have more available water and nutrients than shallow soils, although the relative advantage of deep soils varies with climate, duration of growth season and type of plant (Lambrechts \& Ellis s.d.).

An important soil property to consider in suitability analysis involving vegetation is moisture content as it is an indication of available water for plant use. Water is the major constituent of the physiologically active tissues of plants and serves as a reagent in photosynthetic and hydrolytic vegetation processes. It is also a solvent for salts, sugars and other solutes and is essential for the maintenance of turgidity necessary for cell enlargement and growth (Mweso 2003). Water also alters soil development and its chemical properties, induces periods of drought stress and modifies temperatures that catalyze biotic processes. Owing to its strong relation to topography, soil moisture can be estimated by using indices such as the topographical wetness index (TWI). As a function of upslope area and local slope, TWI is relatively easy to generate from terrain data. Although most types of soil data are valuable for land suitability analysis, soil texture, soil $\mathrm{pH}$, soil effective depth, and TWI were distinguished as fundamental variables for CLUES.

\subsubsection{Terrain types}

Terrain type is used in land evaluation during the land unit mapping phase of the land evaluation process (refer to Section 2.1). Due to its strong relationship with soil and climate, terrain type is often used in the absence of climatic and soil data or as an additional parameter in land suitability assessments (Ceballos-Silva \& López-Blanco 2003b; Cools, De Pauw \& Deckers 2002).

Terrain analysis is the study of the nature, origin, morphological history and composition of landforms, the usual result being a landform map (Argialas 1995). Terrain analysis has wide applications in pure sciences such as hydrology, botany, zoology, and ecology as well as applied sciences such as agriculture, forestry, civil and military engineering, and landscape planning 
(Mitchell et al. 1979). Because landform interpretation and mapping are time consuming, labour intensive and costly operations, and seeing that the skills required are a product of lengthy, expensive training and experience (Argialas 1995), landform maps may be substituted by basic terrain derivatives such as slope gradient, aspect and curvature. These parameters can be generated easily from a digital elevation model (DEM) using standard GIS operations (Van Niekerk \& Schloms 2001).

A DEM records elevations of the earth's surface for each cell in a grid, hereby converting a continuous data variable to a discrete representation (DeMers 2005). This simple model is extremely versatile and highly efficient for computer analysis (Longley et al. 2002).

Slope gradient is defined as the angle between the surface tangent and the horizontal and it controls the gravitational force available for geomorphic work (Van Niekerk \& Schloms 2001). Slope gradient is especially useful for evaluating urban and agricultural land uses as it imposes limitations on construction and cultivation (Lambrechts \& Ellis s.d.; Mitchell 1991). Most governments, including South Africa's, have laws that prevent agricultural and urban developments on steep slopes (James 2001). Slope gradient is also used in environmental modelling owing to the strong relationship between slope gradient and land cover (Adediran et al. 2004; Hoersch, Braun \& Schmidt 2002; Pickup \& Chewings 1996).

Slope aspect is the direction in which a slope faces and therefore determines its exposure to illumination from the sun. In the southern hemisphere, northern slopes receive more solar radiation than southern slopes, especially during winter. Slope aspect, in combination with gradient, determines the amount of solar radiation that reaches an area. It affects the temperature of the soil, the rate of temperature change, vegetation composition, evapotranspiration and other influences on soil properties (Irvin, Ventura \& Slater 1997). Solar radiation is essential for plant development due to its role in photosynthesis making it an important factor to consider in agricultural and forested land uses. Solar radiation also affects the distribution and occurrence of some animal species (Du Toit, Mouton \& Van Niekerk 2006).

Curvature is the rate of change of slope gradient over a given distance and is an indication of where surface runoff will accumulate or disperse. Because of the three-dimensional nature of terrain, slopes can curve in infinite directions. For suitability analysis it is usually sufficient to know whether an area is concave or convex along the slope direction (profile curvature) and/or perpendicular to the slope (plan curvature) (Irvin, Ventura \& Slater 1997; Van Niekerk \& Schloms 2001).

Owing to the developmental limits imposed by slope gradient, the effect of aspect on plant growth (Dendgiz, Bayramin \& Yüksel 2003) and the influence of curvature on hydrological and 
soil formation processes, these two terrain derivatives were confirmed as essential data sets for CLUES. Zhou \& Liu (2004) showed that the accuracy of these derivatives is highly dependent on the quality of the DEM from which they are generated. Care should therefore be taken in the selection of an appropriate DEM.

\subsubsection{Infrastructure attributes}

The availability of existing infrastructure affects the cost and potential to develop land. Many types of infrastructure can be considered in land evaluations. These include roads, railways, airports, electricity, dams, irrigation, and storage facilities. Roads are probably the most important as they provide access to many of the other types of infrastructure. Infrastructure will be incorporated in CLUES by using roads as a fundamental data set.

\subsubsection{Current land cover and use}

The suitability of a parcel of land for a particular land use is affected by its current land cover. Built-up or urban areas, for instance, are less suitable for conservation than wetlands. The land cover classes considered to be of fundamental importance for suitability analysis using CLUES are urban areas, agriculture, wetlands, permanent rivers, and permanent water bodies. Nature conservation areas (consisting of national parks and provincial reserves) were singled out as an important land use to include as a principal data set.

\subsubsection{Summary}

This section aimed to document the functional and operational requirements of CLUES by considering the content of the land evaluation procedure and existing systems. The literature review established that CLUES should be fast enough to enable interactive scenario building, and that a waiting time of less than one minute was appropriate for maps, while other functions should be completed within seven seconds.

The web-based nature of the system poses challenges given that multiple users will access and update the system simultaneously. The system should therefore be designed to allow multiple users access to suitability analyses in such a way that they will be unaware of one another. In addition, user-interface guidelines, as well as web-design principles, must be adhered to in order to ensure that the system is as user-friendly as possible.

A range of land suitability analysis applications were studied to determine what data would be needed to demonstrate the functionality of CLUES. It emerged that data relating to climate, soil, terrain and infrastructure has to be included in the CLUES spatial database. Table 4-1 summarizes the specific data sets identified to be fundamental for inclusion in CLUES. 
Table 4-1 The data requirements of CLUES

\begin{tabular}{|c|c|}
\hline TYPE & REQUIRED DATA SETS \\
\hline Climate & $\begin{array}{l}\text { Annual rainfall } \\
\text { Monthly rainfall } \\
\text { Mean annual temperature } \\
\text { Minimum temperatures (per month) } \\
\text { Maximum temperature (per month) } \\
\text { Mean temperature (per month) } \\
\text { Heat units } \\
\text { Chill units } \\
\text { Frost }\end{array}$ \\
\hline Soil & $\begin{array}{l}\text { Soil effective depth } \\
\text { Soil texture } \\
\text { Soil pH } \\
\text { Topographical wetness index (TWI) }\end{array}$ \\
\hline Terrain & $\begin{array}{l}\text { Elevation (DEM) } \\
\text { Slope gradient } \\
\text { Slope aspect } \\
\text { Slope curvature (plan and profile) }\end{array}$ \\
\hline Infrastructure & Roads \\
\hline $\begin{array}{l}\text { Current land } \\
\text { cover and use }\end{array}$ & $\begin{array}{l}\text { Urban areas } \\
\text { Agriculture } \\
\text { Wetlands } \\
\text { Permanent rivers } \\
\text { Permanent water bodies } \\
\text { Nature conservation areas }\end{array}$ \\
\hline
\end{tabular}

Quality-wise, the data sets used in CLUES for suitability analysis must be as detailed and accurate as possible and must cover the entire Western Cape. Data should preferably be collected at scales ranging between 1:100 000 and 1:30 000 to enable semi-detailed investigations (Lambrechts \& Ellis s.d.), but should be standardized, if possible, at 1:50 000 scale for comparison purposes. This scale is fitting as it is consistent with South Africa's largest scale topographical map series.

Because data collection is not the focus of this research, only existing and available data was considered for inclusion. An inventory and detailed descriptions of the existing climate, soil, terrain, infrastructure, land cover and land use data for the Western Cape are provided in the next chapter. 


\subsection{SYSTEM DESIGN}

CLUES has been designed to demonstrate the potential of the Web as platform for spatial decision support systems. Because web technology has not yet been optimized for spatial analysis, several challenges were presented for system design. The major one was to accommodate the simultaneous data creation and updating needs of spatial analysis in a multiuser web environment. Where GIS software are aimed at individual users, web applications can potentially be accessed by millions of users simultaneously. Special consideration must be given to the effective sharing of computer resources and data. The sharing of data is especially challenging in design because users must be allowed to concurrently edit and update the same data. This is particularly important in land suitability analysis where generic spatial data layers are examined and compared to produce new suitability information. Each suitability analysis generates new data to be managed for each user. Because the establishment of suitability analysis parameters (i.e. land use requirement rules and weights) can be a time consuming process, each user's settings must be managed individually to allow users to build their own rule sets which they can modify and reuse for different projects. The management of data and user settings must be done so that all users are unaware of one another.

To satisfy the requirements set out above, CLUES is designed as a web-based expert system. The design involved combining the components of expert systems with those of web mapping applications. Expert systems that perform suitability analysis usually consist of a land unit database, a knowledge base and an inference engine (see Section 2.4), while web mapping applications (see Section 3.4) normally comprise a spatial database, a WMS and a website. Figure 4-2 shows that all of these components are incorporated into CLUES, with the land unit database acting as the WMS spatial database.

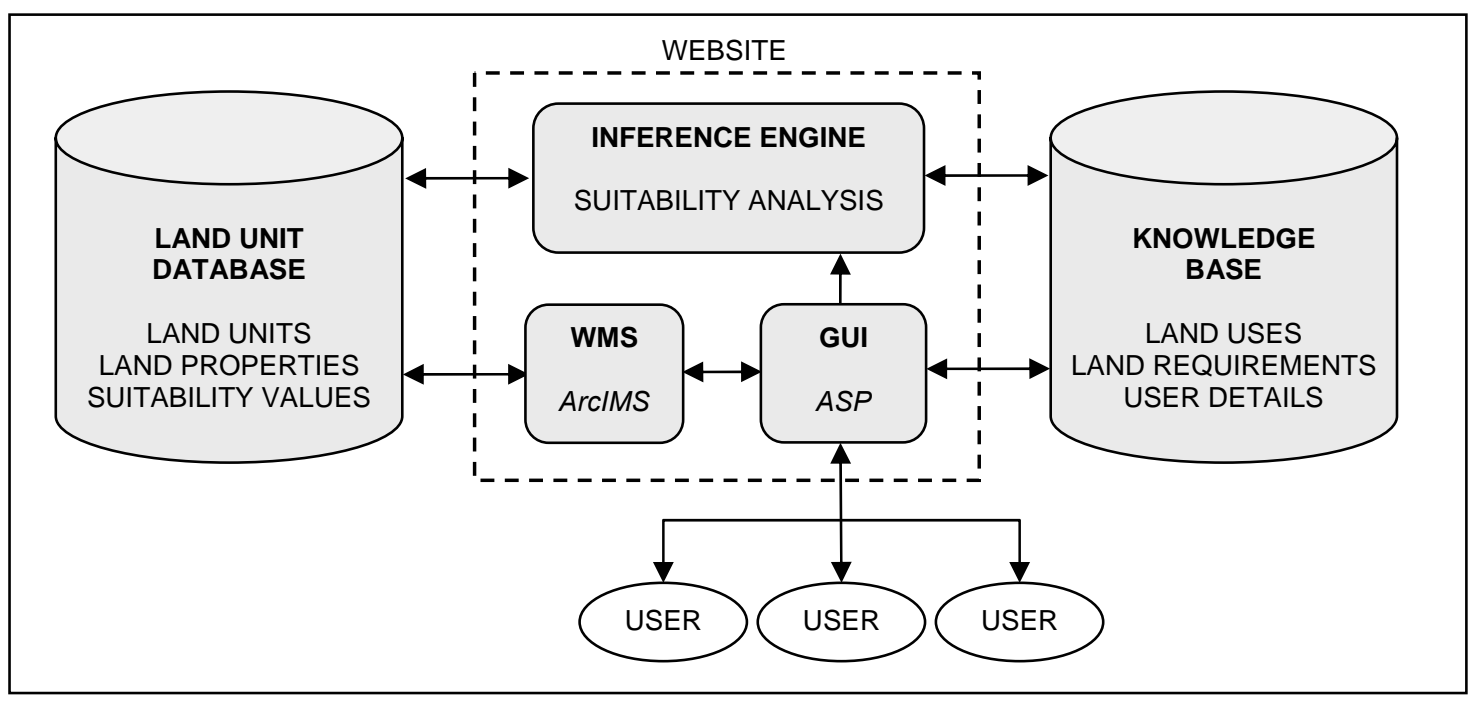

Figure 4-2 The components of CLUES 
As in other expert systems, the two main components of CLUES are the land unit database and the knowledge base. The third major component is the website, which consists of three main elements namely, the graphical user interface (GUI), the web map service (WMS) and the inference engine.

CLUES is designed so that suitability analyses can be done by following the expert system procedure (see Figure 2-9). The function of the GUI is to dynamically generate a collection of web pages through which users can store land requirement rules in the knowledge base and invoke the inference engine to carry out a suitability analysis. During analysis, the inference engine compares the land properties of each land unit in the land unit database with the land requirement rules in the knowledge base. The suitability values calculated by the inference engine are temporarily stored in the land unit database for mapping purposes. To produce suitability maps, the WMS extracts the necessary data from the land unit database and converts the GIS data to dynamic web maps (see Section 3.3.1.1). The maps are temporarily stored on the web server along with the other web page content generated by the GUI. This process is repeated for each suitability analysis.

The suitability analysis procedure is a simplified rendition of the land evaluation procedure. The major difference is that, due to security risk (see Section 3.4.1), CLUES cannot allow users to upload their own spatial data sets. Instead, a collection of existing spatial data sets that are essential to suitability analyses, is available to all users. Should users need specific data for their analyses, it can be loaded by the system administrator.

The system design is based on the principle of loose coupling, a design goal employed in most enterprise and web systems. Loose coupling ensures that a component can be changed or even replaced without affecting the functionality of other components. This is deemed to be important for CLUES because it is expected that the system will be modified and expanded in future. For instance, the two databases (i.e. the land unit database and the knowledge base) are entirely independent of one another. Therefore, the system can easily be applied to another region by simply replacing the land unit database with one of another area. Another advantage of the loose coupling design approach is that it lends flexibility to hardware configuration as changes to hardware will not dramatically affect the individual components.

The implementation of each of the components shown in Figure 4-2 is detailed in the chapters to follow. The first component developed was the land unit database as it contains the spatial data on which the entire suitability analysis is based. To do this, the requisite land property data sets had to be collected. A lack of appropriate data demanded some data manipulations and preparations to be carried out as described in Chapter 5. 
The next implementation activity was to delineate land units so that the land properties could be imported into the database as attributes. Chapter 6 describes the different techniques considered for mapping land units and provides an overview of how the fundamental land properties were assigned to each land unit.

To store the land use requirement rules used to rate the land units according to their suitability for particular uses, the logical data modelling (LDM) procedure was used to design and implement the knowledge base. In addition to the rules, the knowledge base must also accommodate other operational information such as user details and land uses. Chapter 7 describes the LDM procedure for designing and implementing the knowledge base.

The rules in the knowledge base are used by the inference engine to carry out suitability analysis, thus the inference engine acts as an interface between the knowledge base and the land unit database. Users can view and edit the knowledge base through the GUI, but do not have direct access to the land unit database. However, users can access the information in the land unit database through the WMS, which also acts as a type of user interface. In effect, all the components of the website function as a combined interface between the users and the two databases. Each of the elements of the website is described in more detail in Chapter 8.

The suitability maps generated by the inference engine and the WMS are based on the environmental and physical properties contained in the land unit database and the land use requirements in the knowledge base. The next chapter describes the activities to collect and manipulate the appropriate land property data for inclusion in CLUES. 


\section{CHAPTER 5: LAND PROPERTY DATA COLLECTION}

Data collection, the second step in the land evaluation process, involves the capturing, gathering and preparation of the data for use in the suitability analysis. Although there are infinite variables that can be used in suitability assessments (De la Rosa et al. 2004), a number of fundamental data sets relating to climate, soil and terrain were identified during the requirement analysis phase (Section 4.1.3). This chapter describes the activities to acquire the data. The chapter gives an inventory of existing data as well as the motives for selecting specific data sets. The manipulations to prepare the data sets for analysis are also described. The next three sections give details of the selection, collection and manipulation of terrain, soils and climate data.

\subsection{TERRAIN DATA}

Terrain attributes is one of the most useful types of data for land evaluation. Elevation, slope gradient, slope aspect, plan curvature and profile curvature were identified by the requirement analysis to be fundamental data sets for land evaluation. Because all this information can be easily derived from a digital elevation model (DEM), the focus of terrain data collection was to find an appropriate DEM for the Western Cape region. The next three sections concentrate on the criteria for selecting a suitable DEM, inventorying existing models and assessing the accuracy of the chosen DEM respectively.

\subsubsection{DEM selection criteria}

Digital elevation models are essentially elevation rasters generated by interpolating the elevation of a given raster cell from nearby cells with known elevations. The known elevations are typically digitized from topographical maps, but they can also be surveyed elevations (including GPS measurements) obtained using photogrammetry or by processing RADAR (radio detecting and ranging) or LIDAR (light detecting and ranging) data (Campbell 2006; DeMers 2005).

The requirement analysis determined that data only at a map scale of 1:50 000 (or larger) will be considered for inclusion in CLUES. However, because DEM are sometimes derived from primary data sources (such as RADAR and LIDAR), the map scales of DEM are not always known. Map scale is therefore an unsuitable measure for selecting an appropriate DEM for inclusion in CLUES. This also applies to other raster data sets such as climate data (see Section 5.3) derived from primary data sources. In order to select appropriate primary data, raster cells should be smaller than the minimum mapping unit at a scale of 1:50 000. This is specified by McDonald et al (1984) to be 150x150 metres. 
Resolution should not be regarded as the only measure of DEM quality. According to Thompson, Bell \& Butler (2001), the quality of a DEM is influenced by four factors: (1) the interpolation method and algorithm; (2) source data; (3) resolution; and (4) terrain roughness and complexity.

When selecting an interpolation algorithm, the intended use of the DEM and the nature of the source data must be considered. For instance, if the DEM is to be used for small-scale-mapping, a simple inverted linear distance interpolator will suffice, but if the DEM is to be used for hydrological modelling at a local catchment level, a more complex interpolator is required.

Different interpolators are often used for different types of input (source) data due to differences in the density and location of the known elevations. However, some more advanced DEM generation software include several interpolators and can accommodate various source data types, including contours, elevation points, river lines and water bodies as input. Special care must be taken when contours are used as source data because artefacts such as 'rice terraces' and 'tiger stripes' may be created, especially when inferior interpolators are used (Burrough \& McDonnel 1998). The source data also directly influences the quality of the resulting DEM as inaccurate input data will result in an inaccurate DEM - the rule of 'garbage in, garbage out' applies - and minor errors in the source data will be propagated and cause very noticeable artefacts such as spurious sinks and peaks in the DEM (Hengl, Gruber \& Shrestha 2004).

Because resolution can be easily manipulated or changed, it is not always a good measure of the detail contained in a DEM because a high-resolution DEM will not necessarily be more accurate than one at lower resolution derived from the same sample points (Zhou \& Liu 2004). Resolution must be considered in combination with the other three factors (i.e. algorithm, source data, and terrain roughness) when describing a DEM's quality. Since only one elevation value is stored per raster cell, the resolution (i.e. width and height of raster cells) of a DEM has a noticeable influence on the accuracy of a DEM and its derived products (i.e. slope, aspect, curvature). Because each cell occupies a specific area, a reduction in the cell area (i.e. an increase in resolution) can potentially represent surfaces more accurately. This is especially true for terrain with a high degree of topographical complexity as in mountainous areas. One should, however, be aware that a $100 \%$ increase in resolution will result in a $400 \%$ increase in storage volume as the number of cells will increase fourfold.

Due to the lack of a single quantitative measure of quality, absolute vertical accuracy is frequently used to compare DEM. Vertical accuracy is determined by statistically comparing DEM values with known elevations, usually obtained through highly accurate surveying techniques. Another factor that should be taken in consideration when selecting a DEM is the 
accuracy of derived products such as slope gradient and curvature. Thompson, Bell \& Butler (2001) have shown that a low-resolution DEM produces smoother, less-detailed slopes while smaller variations in slopes can be observed when slope gradients are derived from a highresolution DEM. Because slope gradient and curvature are essential for land suitability analysis, vertical accuracy and resolution are both considered when selecting a DEM for CLUES.

\subsubsection{Existing Western Cape DEM}

Three existing DEM were identified that cover the Western Cape. The first is the official South African DEM produced by the Chief Directorate Surveys and Mapping (CDSM) using photogrammetry techniques. The CDSM DEM is not a single DEM, but a combination of three separate DEM with resolutions of 50, 200 and 400 metres respectively. Each of these DEM has a limited coverage that, in combination, covers the entire Western Cape. The 50-metre DEM is available for urban areas only, while the 200-metre DEM only represents mountainous areas. The rest of the Western Cape is covered by the 400-metre DEM. As for quality, CDSM has estimated the vertical accuracy of its product to be 10 metres or better (CDSM 2007a). Unfortunately, due to its variable resolution, the CDSM DEM was disqualified for inclusion in CLUES as the required resolution of at least 150 metres is not met for most of the Western Cape. In addition, the transitions between the three constituent DEM would cause artefacts and unrealistic values if used to derive products such as slope gradient and slope aspect (Thompson, Bell \& Butler 2001).

The second DEM available for the Western Cape is the SRTM DEM, developed in 2000 by NASA during the Shuttle Radar Topography Mission (SRTM) (NASA 2005). In contrast to the variable resolution of the CDSM DEM, the elevation values in the SRTM DEM are regularly spaced at 90-metre intervals, which is considerably better than the minimum requirement of 150 metres. The DEM is also reported to have a vertical accuracy of less than nine metres (Rodriguez et al. 2005).

The third DEM that covers the entire Western Cape is the Western Cape Digital Elevation Model (WCDEM), developed by the Centre for Geographical Analysis (CGA) at Stellenbosch University (Van Niekerk 2001). This 20-metre resolution DEM was generated from contours digitized from the 1:50 000 national topographical map series using ANUDEM software. Although the resolution of the WCDEM is considerably higher than any of the other available DEM, no other information about its accuracy was available. To ensure that the most accurate DEM is included in CLUES, an independent accuracy assessment was conducted on both the WCDEM and the SRTM DEM using the same reference data and methods for both products. 


\subsubsection{DEM accuracy assessment}

To determine the accuracy of the WCDEM and the SRTM DEM, elevation values were systematically compared to reference elevations. Highly accurate (sub-metre) elevation points, obtained from CDSM, were used as reference data. This data was not used in the generation of the WCDEM or SRTM DEM and is therefore a suitable data set to use for the accuracy assessment. To restrict the reference data to a manageable size, a 5\% sample was extracted from the database of reference points. Due to the quarter-degree structure of the database, reference points were selected as blocks of $15 \times 15$ arc minutes. Of the 262 blocks covering the Western Cape, 13 (i.e. 5\%) were selected for the accuracy assessment. To ensure that the selected blocks are representative, a stratified sample was drawn by randomly selecting a proportional number of blocks in each stratum. Regions of one-degree width were used as horizontal strata, resulting in the selection of 2620 reference points. The location of the reference points and the $15 \times 15$ arcminute blocks are shown in Figure 5-1.

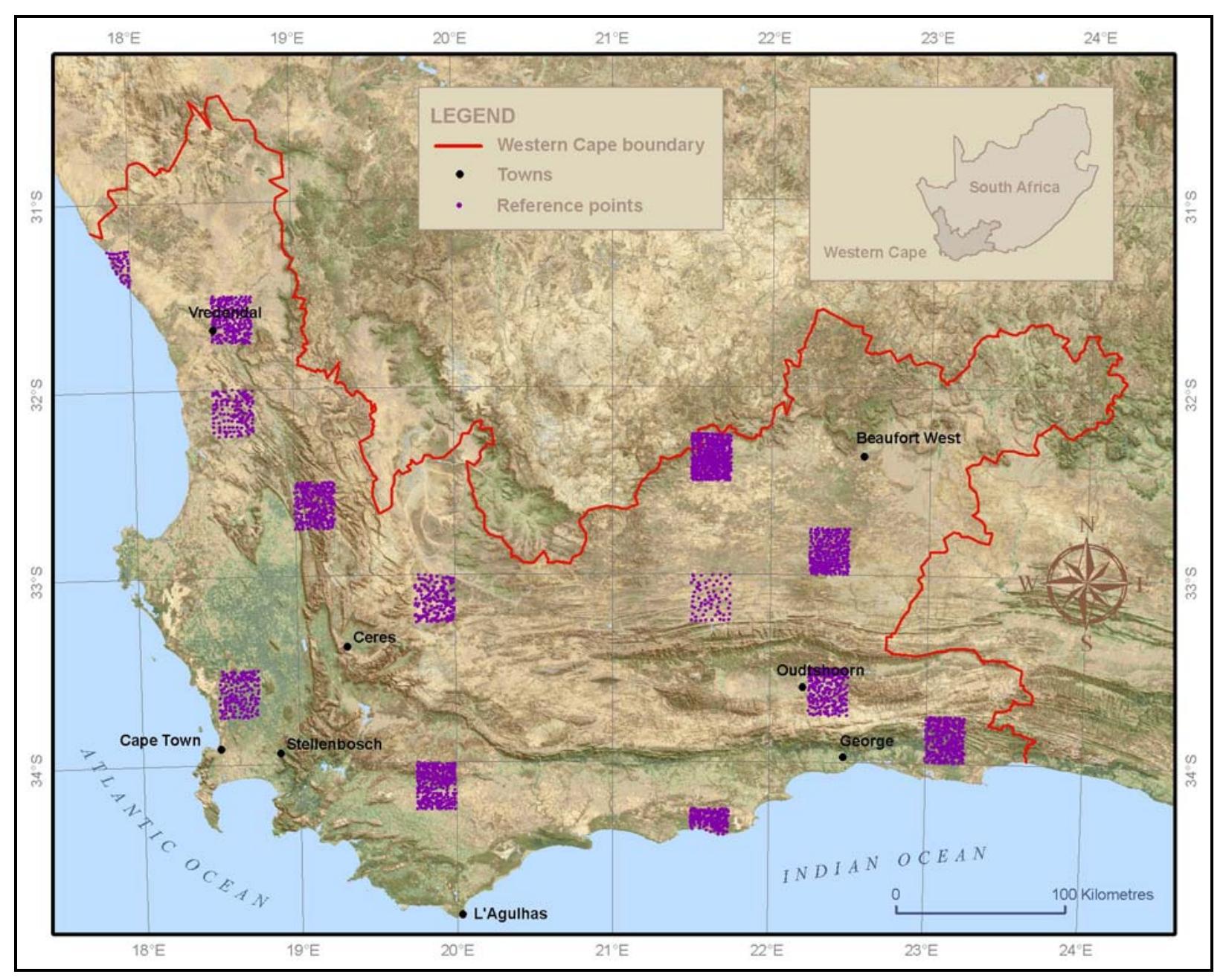

Figure 5-1 Selection of reference points used in the DEM accuracy assessment 
To determine vertical accuracy, the mean absolute error (MAE) and root mean square error (RMSE) for each DEM were calculated using Equations 5-1 and 5-2 respectively (Bolstad \& Smith). The results of the accuracy assessments are summarized in Table 5-1.

$$
M A E=\frac{\sum\left|x_{i}-x_{j}\right|}{n}
$$

where

$$
\begin{array}{cl}
M A E & \text { is the mean absolute error; } \\
x_{i} & \text { is the DEM's elevation value; } \\
x_{j} & \text { is the reference point's elevation value; and } \\
n & \text { is the number of reference points. }
\end{array}
$$

$$
R M S E=\sqrt{\frac{\sum\left(x_{i}-x_{j}\right)^{2}}{n}}
$$

where

RMSE

is the root mean square error;

$x_{i} \quad$ is the DEM's elevation value;

$x_{j} \quad$ is the reference point's elevation value; and

$n \quad$ is the number of reference points.

Table 5-1 Vertical error in the WCDEM and the SRTM DEM

\begin{tabular}{|l|c|c|c|}
\hline DEM & RESOLUTION (m) & MAE (m) & RMSE (m) \\
\hline SRTM DEM & 90 & 15 & 10 \\
\hline WCDEM & 20 & 7 & 10 \\
\hline
\end{tabular}

The WCDEM performed significantly better than the SRTM DEM in terms of MAE and RMSE, both indicators pointing to the WCDEM as the more accurate DEM. Consequently, the WCDEM was selected for CLUES.

Although elevation is a vital land property for suitability analysis, its derivatives are more frequently used in land evaluation. Due to the relatively moderate terrain of the Western Cape (i.e. average slope gradient of $6.5^{\circ}$ ), the Horn algorithm (Chang 2006) available in ArcGIS was used to create slope gradient, slope direction (aspect) and curvature (plan and profile) rasters from the WCDEM.

A data set closely related to terrain and often used in suitability analysis is soil. The next section explores the Western Cape soil data sources. 


\subsection{WESTERN CAPE SOIL INFORMATION}

The importance of soil data for land evaluation was discussed in Section 4.1.3.3. The requirement analysis established that soil type data has little value for suitability analysis and that generic soil properties such as effective soil depth and texture are more useful owing to their quantitative nature. The following sections overview the soil data available for the Western Cape and also describe the preparation of the data for use in CLUES.

\subsubsection{Existing soil data}

According to Lambrechts \& Ellis (s.d.) soil surveys can be categorized according to map scales into detailed (1:1 000 to $1: 2500)$, semi-detailed (1:10 000 to $1: 100000)$, reconnaissance $(1: 120$ 000 to 1:500 000) and investigative $(<1: 500000)$ surveys. Investigative surveys were excluded from the soil inventory as they are usually conducted rapidly along specific routes and are generally unsuitable for land evaluation purposes (Schloms 2007, pers com).

\subsubsection{Detailed surveys}

Detailed soil surveys are expensive to carry out because they involve the collection of extensive field samples and thorough laboratory analysis (McSweeney et al. 1994). Due to the high costs, soil surveys at 1:1 000 to 1:2 500 map scales are usually only conducted for agricultural areas. This is true for the Western Cape where detailed soil maps generally only exist for areas of intensive agriculture (i.e. vineyards and orchards). As most of these surveys are funded by private landowners for farm management and planning purposes, the data is not stored centrally and is therefore not easily accessible (Schloms 2007, pers com).

\subsubsection{Semi-detailed surveys}

Only a limited amount and coverage of semi-detailed soil data is available for the Western Cape. Apart from a peri-urban survey conducted in greater Cape Town at a scale of 1:10 000, a number of 1:50 000 scale surveys have been carried out for the major agricultural areas in the Breede, Berg, Doring and Olifants River catchments. The soils of the rest of the Western Cape remains uncharted at this level of detail (Schloms 2007, pers com).

\subsubsection{Reconnaissance surveys}

Although a number of reconnaissance soil surveys have been conducted for selected areas in the Western Cape (such as the Karoo), a land type survey of South Africa is the only one that covers the entire province. Although this survey was originally conducted at 1:50 000 scale, it was published at a scale of 1:250 000 and cannot be regarded as a semi-detailed survey. 
The land type survey was initiated in 1971 by the Institute of Soil, Climate and Water (ISCW) to provide an inventory of soils, terrain forms and macro-climate for South Africa. It was completed in 2002. Funded by the National Department of Agriculture (NDA), the survey was based on factual observations, soil analysis and long-term climatic records so that it can be reliably applied to determine land use potential, land sustainability, and best management practices (Patterson 2005; Turner 2005).

\subsubsection{Soil database strategy}

The soil inventory exercise established that detailed soil data of the Western Cape is lacking and that data at the required scale of 1:50 000 is available for certain areas only. Because CLUES requires a continuous layer of soil data covering the entire province, the land type data was chosen as the fundamental soil data source in spite of its generalized nature. This data will be substituted by more detailed information when it becomes available. This strategy means that differently scaled soil data will be used for different areas - a less than ideal option. However, it does ensure that a suitability analysis conducted anywhere in the Western Cape is based on the best available data.

The next section overviews the land type data and documents how it was manipulated to extract soil information in a suitable format for land evaluation purposes.

\subsubsection{Land types information system}

The ISCW land type information is published as memoirs. An example is the memoir for land type $\mathrm{Ca} 6$ as shown in Figure 5-2. This specific land type covers an area of 421200 hectares and includes four terrain units. Soil information is related to each terrain unit as illustrated by the terrain form sketch. Each terrain unit is further described in terms of its area, slope, slope length, slope shape, mechanical limitations and soil series. By interpreting this information, a soil scientist can easily gain a synopsis of the soils in each land type. The land type data was never intended for computer analysis and is therefore unsuited for making quantitative comparisons between land types using GIS. There is, for instance, no easy way to find all the land types having soils deeper than one metre. Consequently, innovative manipulations are needed to quantify the land type data. To do so, knowledge of the data structure of the recently digitized data set was required. 


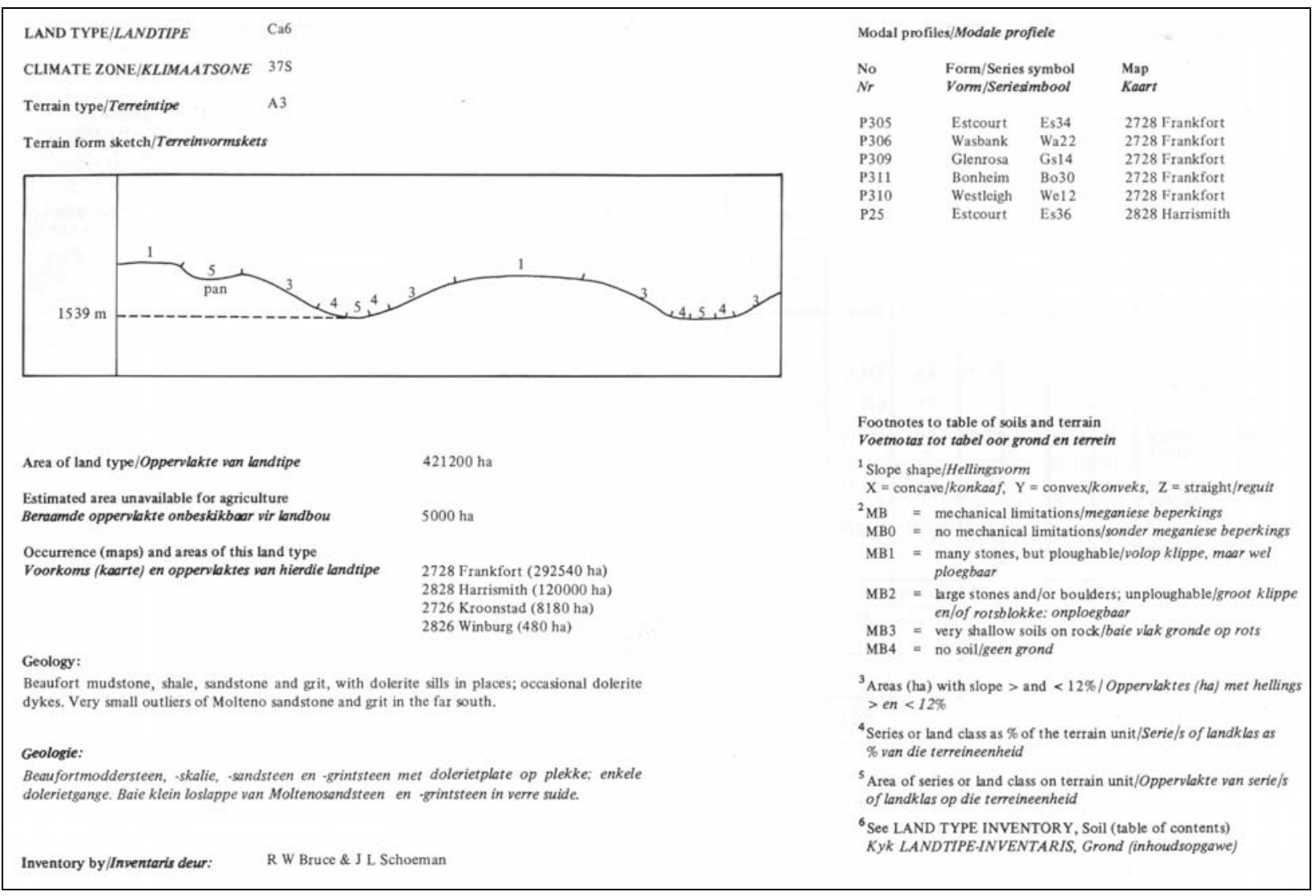

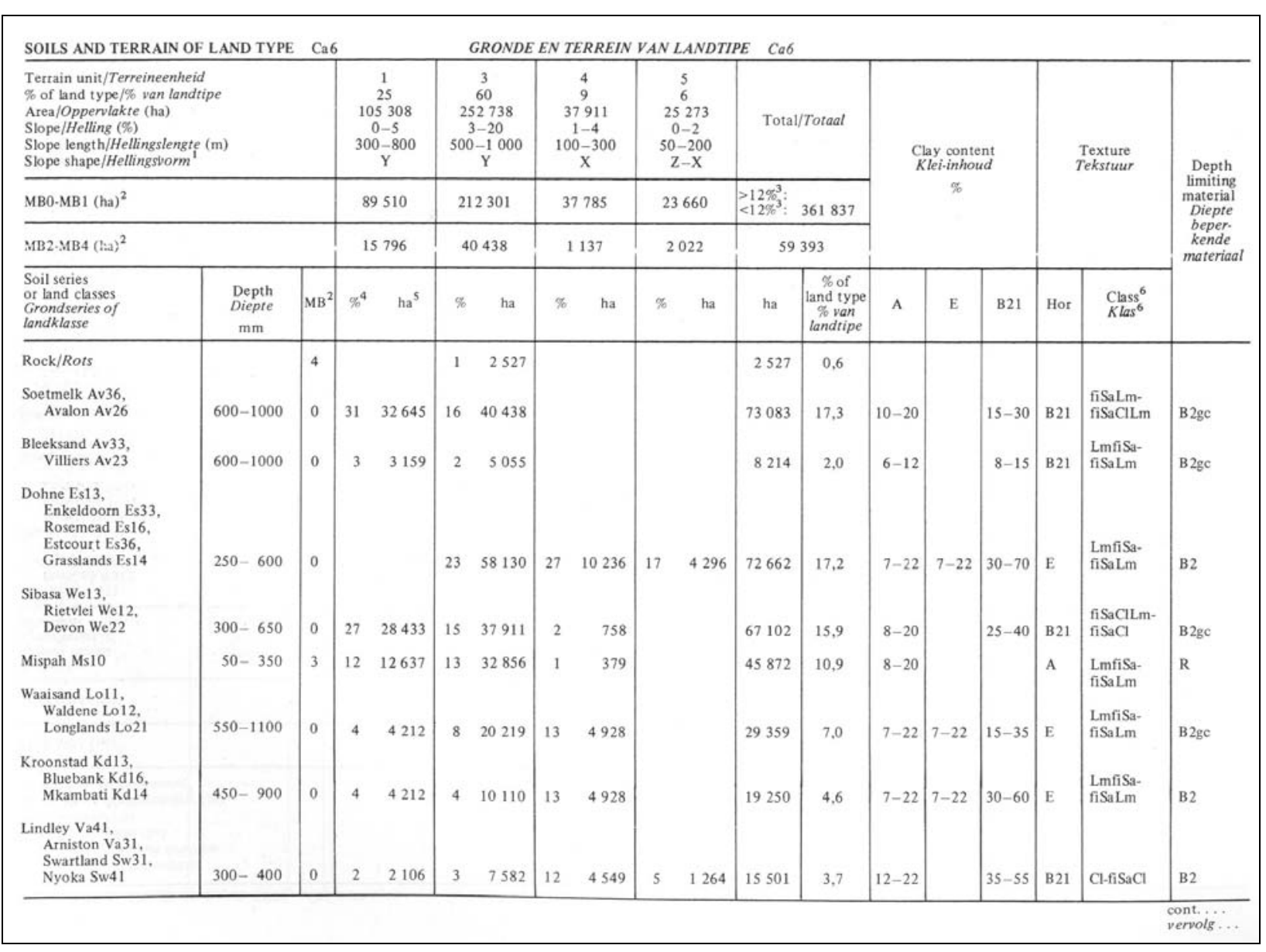

Figure 5-2 Land type Ca6 in memoir format 


\subsubsection{Digital data structure}

The digital land type data consists of spatial and tabular components. The spatial component encompasses the location and boundaries of each land type, stored as polygons (see Figure 5-3a) in shape file format. The spatial features are linked to an attribute table (Figure 5-3b) which, in turn, can be linked to six informational tables, namely Tables A, B, D, E, F, and G shown in Figure 5-4.

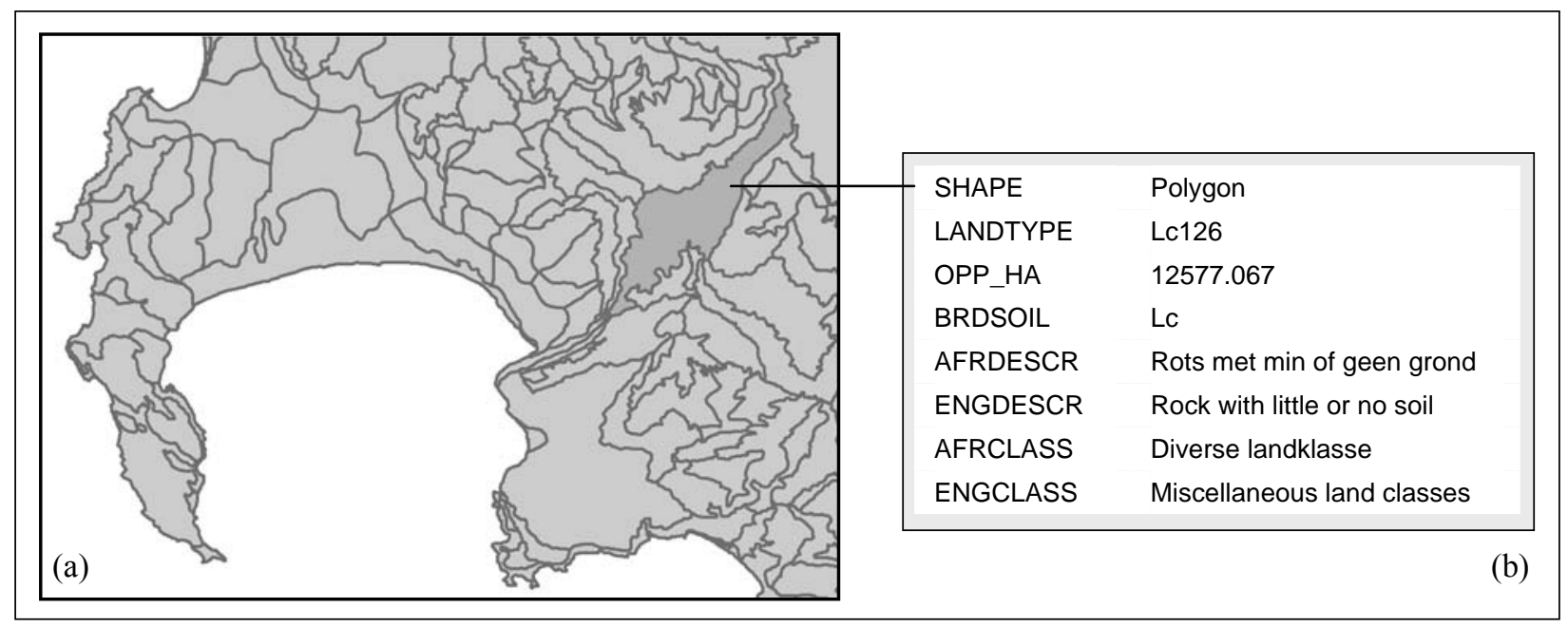

Figure 5-3 Land type polygon (a) and its associated attribute information (b)

\begin{tabular}{|c|c|c|c|c|c|c|c|}
\hline & & & ATTRIBUTES & \multirow{2}{*}{$\mathrm{m}-$} & & & TABLE F \\
\hline TABLE B & TABLE A & \multirow{8}{*}{$\mathrm{m}$} & \multirow{18}{*}{$\begin{array}{l}\text { LANDTYPE } \\
\text { BRDSOIL } \\
\text { AFRDESCR } \\
\text { ENGDESCR } \\
\text { AFRCLASS } \\
\text { ENGCLASS } \\
\text { OPP_HA }\end{array}$} & & \multirow{3}{*}{$\begin{array}{l}\text { TABLE D } \\
\text { LANDTYPE } \\
\text { TERRAIN_U }\end{array}$} & & LANDTYPE \\
\hline LANDTYPE & LANDTYPE & & & \multirow{17}{*}{$\mathrm{m}$} & & & \multirow{9}{*}{$\begin{array}{l}\text { COMPLEX } \\
\text { SERIES } \\
\text { SOIL_D_L } \\
\text { SOIL_D_T } \\
\text { SOIL_D_U } \\
\text { CLAY_A_L } \\
\text { CLAY_A_T } \\
\text { CLAY_A_U } \\
\text { CLAY_E_L } \\
\text { CLAY_E_T }\end{array}$} \\
\hline MAPNO & CLIMATENO & & & & & & \\
\hline MAPAREA & TERRAIN_T & & & & TERRAIN_P & & \\
\hline & AREA & & & & SLOPE_L & & \\
\hline TABLE E & UNAREA & & & & SLOPE_T & & \\
\hline LANDTYPE & INVENTORY & & & & SLOPE_U & & \\
\hline PROFILE & & & & & SLOPE_LL & & \\
\hline & & & & & $\begin{array}{l}\text { SLOPE_IL } \\
\text { SIOPF III }\end{array}$ & & \\
\hline & & & & & SLOPE_SHP & & \\
\hline & & & & & & & CLAY_E_U \\
\hline & & & & & & & CLAY_B_L \\
\hline & & & & & & & CLAY_B_T \\
\hline & & & & & TABLE G & & CLAY_B_U \\
\hline & & & & & LANDTYPE & $\mathrm{m}$ & DEPTH_L \\
\hline & & & & & TERRAIN_U & & \\
\hline & & & & & SOIL_P & & HORIZON \\
\hline & & & & & SKAKEL & & SKAKEL \\
\hline
\end{tabular}

Figure 5-4 Land type database structure

As with any shape file, the SHAPE field in the attribute table (Figure 5-3b) contains objects that define the shape and position of each polygon (ESRI 2002b). Each land type consists of one or more polygons and can be uniquely identified through the land type code stored in the LANDTYPE field. The OPP_HA field contains the area in hectares of each polygon while the 
remaining fields BRDSOIL, LAFRDESCR, ENGDESCR, AFRCLASS, and ENGCLASS provide general descriptive information bilingually.

The LANDTYPE field in the attribute table relates to Table A in Figure 5-4 containing information about the climate (CLIMATENO), general terrain type (TERRAIN_T), area available for agriculture (AREA) and area unavailable for agriculture (UNAREA), as well as the name of the surveyor who inventoried (INVENTORY) each land type. Table A is related one-tomany with the attribute table; in other words, every row in Table A is related to many rows (polygons) in the attribute table through its primary key, LANDTYPE.

Table B stores information about the original land type map sheet (MAPNO) from which each land type was digitized. As a land type could have been digitized from one or more sheets, this table has a many-to-one relationship with Table A. When combined, the LANDTYPE and MAPNO fields act as a primary key. The table also stores the area each land type covers on the original sheet or sheets in MAPAREA.

Similarly, Table E relates many-to-one to Table A. In Table E the modal profiles (PROFILE) of each land type are stored, providing a general indication of the type of soil occurring in a land type.

Information about a land type's terrain units is stored in Table D. This table has a many-to-many relationship with the attribute table as multiple polygons representing any land unit can relate to multiple terrain units (TERRAIN_U field). The LANDTYPE field in Table D is therefore not unique. Rows in Table D can, however, be uniquely identified by combining the LANDTYPE and TERRAIN_U fields. The percentage area that each terrain unit covers is stored in the TERRAIN_P field. The slope gradient (\%) is stored as a range in fields SLOPE_L, SLOPE_T and SLOPE_U, where the suffix L indicates lower limit, $\mathrm{T}$ the operand $(<,-,>)$ and $\mathrm{U}$ the upper limit. Similarly slope length (in metres) is stored in fields SLOPE_LL, SLOPE_TL and SLOPE_UL. The SLOPE_SHP field indicates whether the shape of the slope is convex, straight, or concave.

Tables $G$ and $F$ store information about each soil series found in each terrain unit (TERRAIN_U). Table G can be linked many-to-one to Table D using the LANDTYPE and TERRAIN_U fields combined and Table F is related many-to-one by the SKAKEL field. Table F contains details of each soil series, including effective soil depth, clay content per horizon and mechanical limitations. Similar to slope in Table D, effective soil depths (mm) are stored as ranges in fields SOIL_D_L, SOIL_D_T and SOIL_D_U, while CLAY_A_L, CLAY_A_T and CLAY_A_U store clay content (\%) in the A horizon in Table F. The clay content of horizons E and B21 is provided in the same manner in CLAY_E_L, CLAY_E_T, CLAY_E_U, 
CLAY_B_L, CLAY_B_T, and CLAY_B_U. The clay content of horizons E and B21 is absent for many land types.

In addition, mechanical limitations are stored in the MB field as integers ranging from 0 to 4 indicating no mechanical limitations (0); many stones, but ploughable (1); large stones and/or boulders, unploughable (2); very shallow soils on rock (3); and no soil (4).

Figure 5-5 shows how this structure can be conceptualized in levels of detail. Each land type (LT) can have one or more terrain units (TU) in level 2 and each terrain unit can have one or more soil series (SS) in level 3. A terrain unit cannot be shared between two land types, but a soil series $\left(\mathrm{SS}_{\mathrm{j}}\right)$ can occur on two different terrain units $\left(\mathrm{TU}_{\mathrm{i}}\right.$ and $\left.\mathrm{TU}_{\mathrm{j}}\right)$ if they occur in the same land type $\left(\mathrm{LT}_{\mathrm{i}}\right)$. Only level 1 has a spatial component. Terrain and soil information must therefore be extracted from the tabular data and applied at the land type level in order for the data to be suitable for map production or analysis. The extraction procedure is explained in the next section.

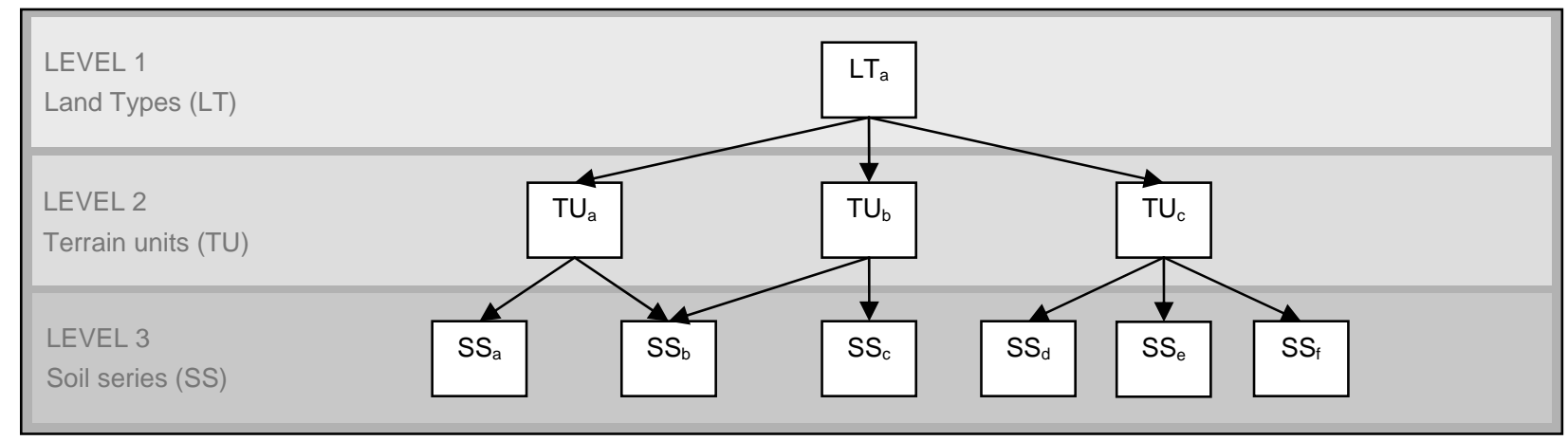

Figure 5-5 Conceptual view of land type object levels

\subsubsection{Soil property extraction}

The land type data contains soil property information in the form of effective depth, clay content, and mechanical limitations. Although the requirement analysis highlighted soil texture as a fundamental variable in land evaluation, no information about texture is available in the land type data. However, clay content can be used as a surrogate for texture as texture tends to decrease with increasing levels of clay.

Because it is not possible to directly link effective depth, clay content, and mechanical limitations to the land type polygons, the land type data is not suitable for mapping or spatial analysis. This section provides an overview of how the various soil properties were extracted from the land type data for quantitative land suitability analysis. 


\section{Effective soil depth}

Effective soil depth is stored at the soil series level (see Figure 5-5), so to use this information for mapping or spatial analysis, the effective depths of all the soil series within a land type must be aggregated. This is achieved by using simple averaging techniques at all three data levels. The first step converts each effective soil depth range in Table $\mathrm{F}$ to its central value using Equation 5-3. The result is a value representing the average effective soil depth of each soil series.

$$
D_{s}=\frac{D_{s}^{\max }-D_{s}^{\min }}{2}
$$

Equation 5-3

where $\quad D_{s} \quad$ is the average effective soil depth of soil series $s$;

$D_{s}^{\max } \quad$ is the upper value of the effective depth range for soil series s; and

$D_{s}^{\min } \quad$ is the lower value of the effective depth range for soil series s.

Next, the average effective soil depth of each terrain unit is calculated. In Equation 5-4 the individual $D_{s}$ values are summed using the percentage area (SOIL_P) of each soil series as weights.

$$
D_{t}=\sum_{s=1}^{n}\left(D_{s} \times \frac{P_{s}}{100}\right)
$$

Equation 5-4

where $\quad D_{t} \quad$ is the average effective soil depth of terrain unit $t$;

$D_{s} \quad$ is the average effective soil depth of soil series $s$;

$P_{s} \quad$ is the percentage area covered by soil series $s$ within terrain unit $t$; and

$n \quad$ is the number of soil series within terrain unit $t$.

To calculate the average effective soil depth of the entire land type, Equation 5-5 is applied. Similar to Equation 5-4, the relative size of each terrain unit is considered in the summation.

$$
D_{l}=\sum_{t=1}^{m}\left(D_{t} \times \frac{P_{t}}{100}\right)
$$

Equation 5-5

where $\quad D_{l} \quad$ is the average effective soil depth of land type $l$;

$D_{t} \quad$ is the average effective soil depth of terrain unit $t$;

$P_{t} \quad$ is the percentage area covered by terrain unit $t$ within land type $l$; and

$m \quad$ is the number of terrain units within land type $l$.

By substituting Equation 5-3 and Equation 5-4 into Equation 5-5 it follows that:

$$
D_{l}=\sum_{t=1}^{m}\left(\sum_{s=1}^{n}\left(\frac{D_{s}^{\max }-D_{s}^{\min }}{2} \times \frac{P_{s}}{100}\right) \times \frac{P_{t}}{100}\right)
$$

Equation 5-6

Equation 5-6 could only be used for soil series with effective soil depth values in both the SOIL_D_L and SOIL_D_U fields in Table F. Some records only contain values in the 
SOIL_D_U field with a ' $>$ ' or ' $<$ ' operator in the SOIL_D_T field to indicate that the effective soil depth for the particular soil series is greater or smaller than the SOIL_D_U value. In cases where the operator is ' $<$ ', the SOIL_D_U value was halved, while the SOIL_D_U value was used as the soil series depth where ' $>$ ' occurred in the SOIL_D_T field. These exceptions were handled programmatically.

Only effective soil depths of $1200 \mathrm{~mm}$ or less are specified in the land type data. Deeper soils are indicated as being deeper than $1200 \mathrm{~mm}$. Because the extraction process considered such soils as being only $1200 \mathrm{~mm}$ deep, the averaging effect of the procedure underestimates true depths. Values of more than $1000 \mathrm{~mm}$ should therefore be considered to be ' 1000 or deeper'.

The result of the soil property extraction procedure is a single value representing average effective soil depth for each land type. In this format the effective depth information is more suitable for spatial analysis. It can also be used to produce a choropleth map (see Figure 5-6) of effective soil depth in the Western Cape. The map confirms the notion that deeper soils occur on valley bottoms and plains, while shallow soils are more frequently found in areas with high relief.

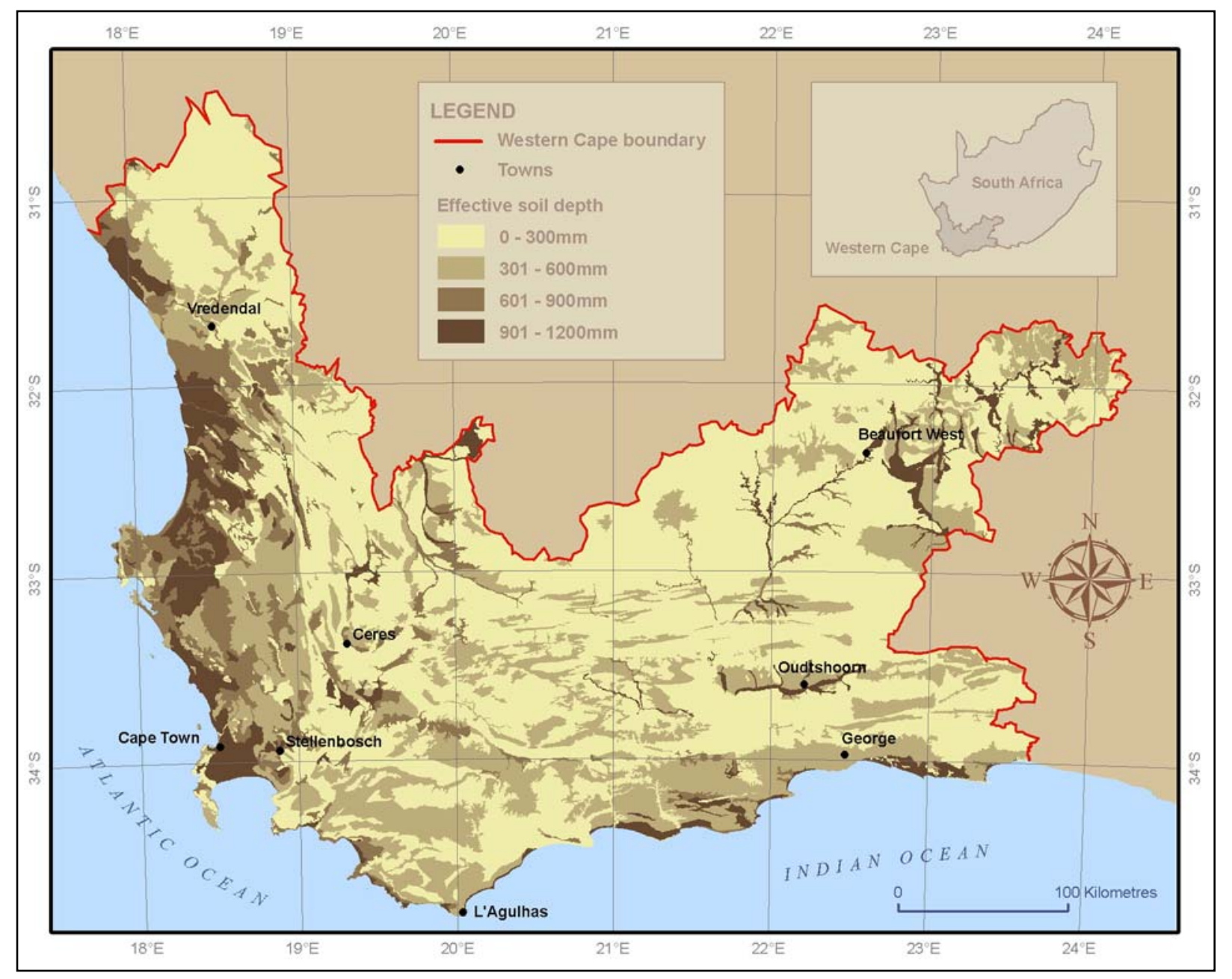

Figure 5-6 Effective soil depth derived from land type data 
Average clay content

To calculate the average clay content of each land type, only the A horizon was considered as B and $\mathrm{E}$ horizons are not always present. Moreover, duplex soils may cause misleading results. As seen in Equation 5-7, the procedure to calculate the average clay content of horizon $\mathrm{A}$ is almost identical to that for effective depth (Equation 5-6). The spatial extraction result is shown in Figure 5-7.

$$
C_{l}=\sum_{t=1}^{m}\left(\sum_{s=1}^{n}\left(\frac{C_{s}^{\max }-C_{s}^{\min }}{2} \times \frac{P_{s}}{100}\right) \times \frac{P_{t}}{100}\right)
$$

where $\quad C_{l} \quad$ is the average clay content of horizon A;

$C_{s}^{\max } \quad$ is the upper value of the A horizon clay content range for soil series $s$;

$C_{s}^{\min } \quad$ is the lower value of the A horizon clay content range for soil series $s$;

$P_{s} \quad$ is the percentage area covered by soil series $s$ within terrain unit $t$;

$n \quad$ is the number of soil series within terrain unit $t$;

$P_{t} \quad$ is the percentage area covered by terrain unit $t$ within land type $l$; and

$m \quad$ is the number of terrain units within land type $l$.

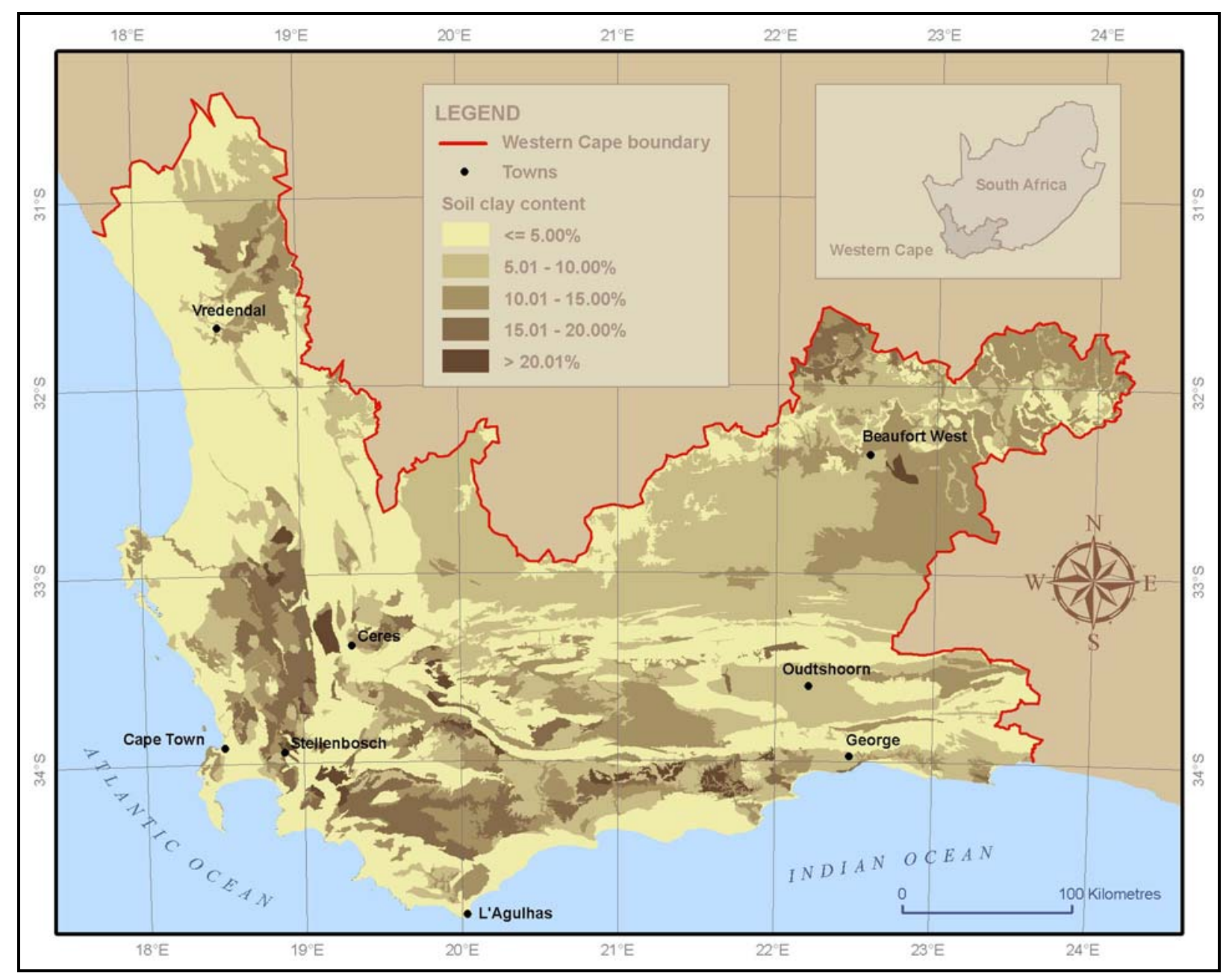

Figure 5-7 Soil clay content derived from land type data 


\section{Mechanical limitations}

Equation 5-8 is used to calculate an average mechanical limitations value $\left(M_{l}\right)$ for each land type and the spatial result is displayed in Figure 5-8. The only difference between this procedure and those followed for effective soil depth and soil clay content is that the mechanical limitations of each series $\left(M_{S}\right)$ are stored as integers (0-4) and not as ranges so that no conversion is necessary.

$$
M_{l}=\sum_{t=1}^{m}\left(\sum_{s=1}^{n}\left(\frac{M_{s}}{2} \times \frac{P_{s}}{100}\right) \times \frac{P_{t}}{100}\right)
$$

Equation 5-8

where $\quad M_{S} \quad$ is the mechanical limitations value for soil series $s$;

$P_{s} \quad$ is the percentage area covered by soil series $s$ within terrain unit $t$;

$n \quad$ is the number of soil series within terrain unit $t$;

$P_{t} \quad$ is the percentage area covered by terrain unit $t$ within land type $l$; and

$m \quad$ is the number of terrain units within land type $l$.

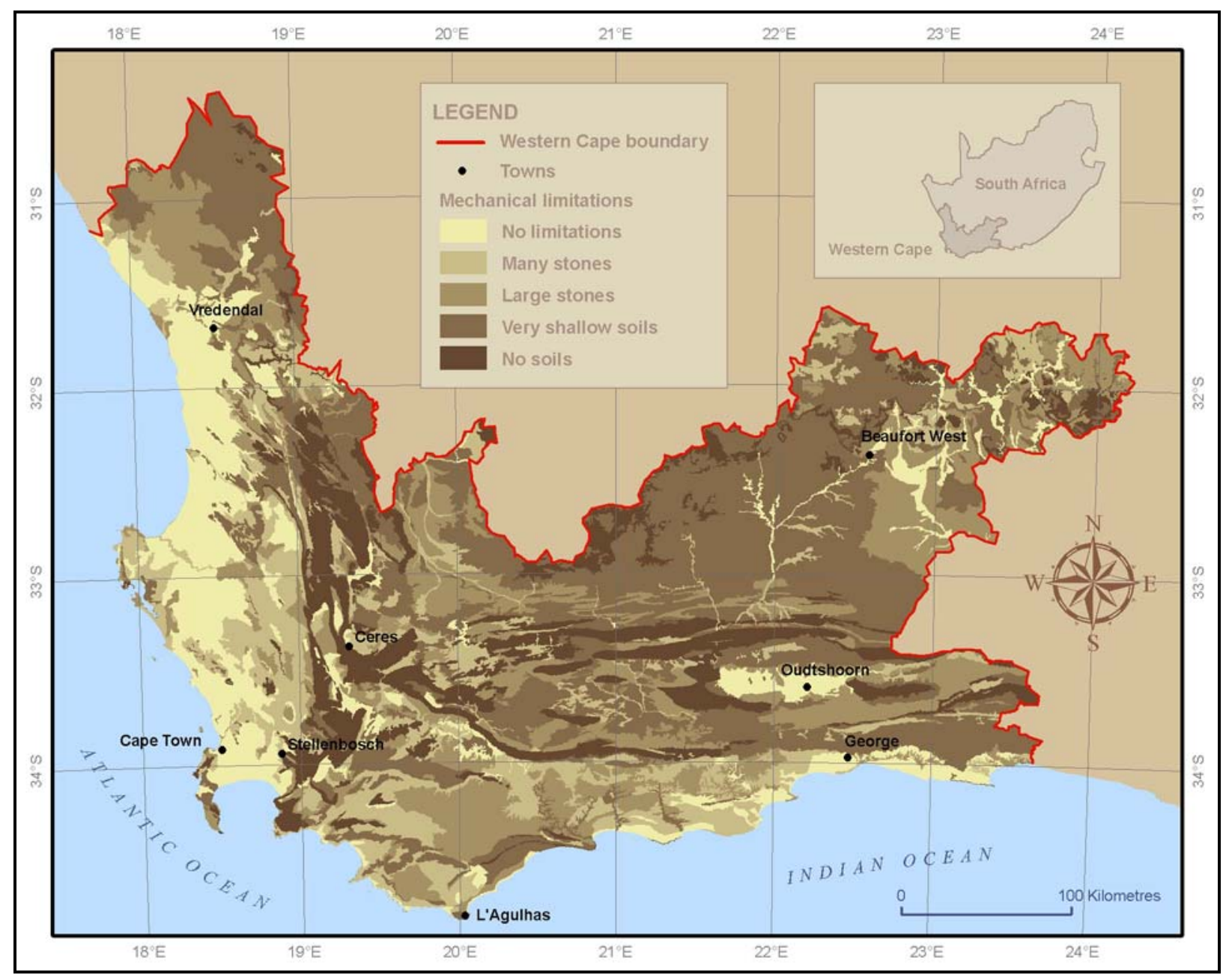

Figure 5-8 Soil mechanical limitations derived from the land type data

To manually carry out the calculations necessary to extract the soil properties discussed above for all the land types is a time-consuming, if not impossible, task. Although GIS can speed up the process, hundreds of GIS operations would be required to retrieve and combine the necessary 
data from the six tables in Figure 5-4. This would not only be a time-consuming task, but is prone to human error. An alternative - automating the entire procedure using programming techniques - was implemented. An Avenue script (see Appendix A) was written and executed in ArcView GIS 3.3. The automation not only saves time, but enables easy repetition. It can also be used to extract soil property information from the data base for the rest of South Africa.

Although the land type survey includes general climatic indicators, an inventory of other available climate data sources for the Western Cape was made.

\subsection{WESTERN CAPE CLIMATE INFORMATION}

According to Lutgens \& Tarbuck (1998), an area's climate is an aggregate of its weather conditions over time. To derive reliable climate data, statistical analysis is performed on longterm (i.e. 30 years or more) weather observations (Houghton et al. 2001). In South Africa, weather data is recorded by the South African Weather Services (SAWS) through a national network of 118 automatic weather stations, 112 climate stations and 1512 rainfall stations. Unfortunately, many of the weather stations are sparsely situated, especially in mountainous regions or areas with low population densities, resulting in vast regions being insufficiently represented by weather stations (SAWS 2007). Interpolation methods are employed to estimate climate data for areas not represented by weather stations. The accuracy of such estimations is a function of the distance between weather stations and the interpolation method employed. Based on the distance averaging effect, a higher density of weather stations should result in greater accuracy. Accuracy is also influenced by the interpolation algorithm used (Lynch 1999). These factors were considered during the selection and capture of appropriate climate data for inclusion in CLUES.

\subsubsection{Existing climate data}

The most commonly used climatic data for South Africa was developed by Schulze (1997) for the South African atlas of agrohydrology and-climatology (SAAAC). The SAAAC data was derived from weather station observations at a resolution of one arc minute $(\approx 2 \mathrm{~km})$ using stepwise multiple regression techniques. Latitude, longitude, altitude, and aspect were included to produce a statistical goodness-of-fit model for each quaternary catchment in South Africa. The modelled values were combined to produce more than 200 climatic and agrohydrological data sets, including mean annual precipitation and median monthly rainfall, as well as means of daily maximum and minimum temperature, on a national scale (Schulze 1997). 
A second global set of climatic data was developed for WorldClim by Hijmans et al. (2005) at a resolution of 30 arc seconds $(\approx 1 \mathrm{~km})$. The WorldClim grids were interpolated from weather station observations using ANUSPLIN software, the variations between weather stations being modelled using latitude, longitude and elevation as independent variables. The authors showed that there is significant benefit in the higher spatial resolution of their climate grids. This is mainly because a higher spatial resolution can accommodate more variation in terms of terrain.

The influence of resolution on terrain variability led to the development of a set of high resolution climatic grids for the Western Cape (Joubert \& Van Niekerk 2005). Long-term (35 years on average) weather data was collected for 125 weather stations in and around the province and used as the data source for the interpolation of the Western Cape climate grids (WCCG). Combinations of elevation, latitude, longitude, hillshade and distance to oceans were used as covariates and independent variables in the ANUSPLIN interpolation algorithm. To model as much climatic variation as possible while keeping data sets manageably small, the SRTM DEM (see Section 5.1.2) was used as the data source for elevation and hillshade. Climate grids at a resolution of 90 metres were interpolated for monthly mean daily maximum temperature, monthly mean daily minimum temperature and mean monthly rainfall (Joubert 2007).

\subsubsection{Comparison of data quality}

According to the requirement analysis, climate data is needed at a 1:50 000 map scale. Because climate grids are interpolated from weather station point data, map scale is not an appropriate measure of suitability. Instead, a comparison of the existing grids regarding their spatial resolution and accuracy was made.

The reported accuracies of the existing data are summarized in Table 5-2. Other than the slight difference in resolution between the SAAAC and WorldClim data sets, no difference in accuracy was observed between these two data sources. The WCCG data set is significantly more accurate than the other two data sets - three times more accurate in terms of temperature and more than twice as accurate in terms of rainfall.

Table 5-2 Accuracy summary of existing climatic data for the Western Cape

\begin{tabular}{|l|c|c|c|}
\hline SOURCE & RESOLUTION $(\mathrm{m})$ & TEMPERATURE $\left({ }^{\circ} \mathrm{C}\right)$ & RAINFALL (mm) \\
\hline SAAAC & 1600 & 1.0 & 10.0 \\
\hline WorldClim & 1000 & 1.0 & 10.0 \\
\hline WCCG & 90 & 0.3 & 4.5 \\
\hline
\end{tabular}


The markedly higher accuracy of the WCCG data is attributable to the inclusion of more source data (weather stations), the use of more-detailed elevation data, and the incorporation of more independent variables into the interpolation algorithm. In addition, with its resolution of 90 metres, the WCCG data set is more than ten times finer than the other two sets. This higher level of detail is especially important for suitability analysis of land uses such as perennial crops that are strongly influenced by local (micro) climate. Understandably, the WCCG data set was chosen as the primary climate data source for CLUES. In addition to the WCCG data, other climate data sets namely heat units, chill units and frost were included. These data were considered essential for agrohydrological applications of CLUES.

\subsection{SUMMARY}

This chapter described the collection of suitable terrain, soil and climate data needed to demonstrate the functionality of CLUES. A selection was made from existing data sets by comparing data quality and scale. Concerning terrain, the WCDEM is presently the most accurate DEM available for the Western Cape, while the WCCG climate data is notably superior in comparison to other available climate data. A summary of the data sets to be used in CLUES is given in Table 5-3.

Table 5-3 Data sets collected for CLUES

\begin{tabular}{|c|c|c|c|}
\hline TYPE & DATA SET & SOURCE & RESOLUTION/SCALE \\
\hline Climate & $\begin{array}{l}\text { Annual rainfall } \\
\text { Monthly rainfall } \\
\text { Mean annual temperature } \\
\text { Minimum temperature (per month) } \\
\text { Maximum temperature (per month) } \\
\text { Mean temperature (per month) } \\
\text { Heat units } \\
\text { Chill units } \\
\text { Frost }\end{array}$ & $\begin{array}{l}\text { Joubert (2007) } \\
\text { Joubert (2007) } \\
\text { Joubert (2007) } \\
\text { Joubert (2007) } \\
\text { Joubert (2007) } \\
\text { Joubert (2007) } \\
\text { Schulze (1997) } \\
\text { Schulze (1997) } \\
\text { Schulze (1997) }\end{array}$ & $\begin{array}{l}90 \mathrm{~m} \\
90 \mathrm{~m} \\
90 \mathrm{~m} \\
90 \mathrm{~m} \\
90 \mathrm{~m} \\
90 \mathrm{~m} \\
\approx 2 \mathrm{~km} \\
\approx 2 \mathrm{~km} \\
\approx 2 \mathrm{~km}\end{array}$ \\
\hline Soil & $\begin{array}{l}\text { Effective soil depth } \\
\text { Soil texture } \\
\text { Topographical wetness index (TWI) }\end{array}$ & $\begin{array}{l}\text { Land Type Survey Staff (1984) } \\
\text { Land Type Survey Staff (1984) } \\
\text { Van Niekerk (2001) }\end{array}$ & $\begin{array}{l}1: 250000 \\
1: 250000 \\
20 m\end{array}$ \\
\hline Terrain & $\begin{array}{l}\text { Elevation (DEM) } \\
\text { Slope gradient } \\
\text { Slope aspect } \\
\text { Slope curvature (plan and profile) }\end{array}$ & $\begin{array}{l}\text { Van Niekerk (2001) } \\
\text { Van Niekerk (2001) } \\
\text { Van Niekerk (2001) } \\
\text { Van Niekerk (2001) }\end{array}$ & $\begin{array}{l}20 m \\
20 m \\
20 m \\
20 m\end{array}$ \\
\hline Infrastructure & Roads & CDSM (2007b) & $1: 50000$ \\
\hline $\begin{array}{l}\text { Current land } \\
\text { cover and use }\end{array}$ & $\begin{array}{l}\text { Urban areas } \\
\text { Wetlands } \\
\text { Permanent rivers } \\
\text { Permanent waterbodies } \\
\text { Nature conservation areas }\end{array}$ & $\begin{array}{l}\text { Thompson (1999) } \\
\text { Thompson (1999) } \\
\text { Thompson (1999) } \\
\text { Thompson (1999) } \\
\text { Thompson (1999) }\end{array}$ & $\begin{array}{l}1: 250000 \\
1: 250000 \\
1: 250000 \\
1: 250000 \\
1: 250000\end{array}$ \\
\hline
\end{tabular}


There is a paucity of soil data for the Western Cape, land type data being the only data set that meets the required coverage. However, because the land type data is published at a scale of 1:250 000, it does not satisfy the 1:50 000 scale requirements. In the absence of better data, land type information is included as a fundamental soil data layer, with the recommendation that the generic soil properties (i.e. effective soil depth, texture and $\mathrm{pH}$ ) be updated with more detailed soil data as it becomes available.

The data sets listed in Table 5-3 were collected to be used as land property information. Based on the land evaluation approach, land properties are related to land use requirements to determine whether a land unit is suitable for a particular use. As explained in Section 4.2, land properties are stored as attributes of land units in the land unit database. The activities to develop the land unit database are described in the next chapter. 


\section{CHAPTER 6: DEVELOPING THE LAND UNIT DATABASE}

The first step to implement CLUES was to set up a land unit database as a repository of all the spatial data required for suitability analysis using CLUES. The database essentially consists of polygons (land units) with a set of attributes (land properties).

Land units were defined in Section 2.1.5 as parcels of land that differ significantly from the surrounding land. Although any parcel of land can be considered a land unit, it is more efficient and meaningful to use parcels that can be adequately described by one or a combination of land properties. A land unit should represent an area that, according to predetermined properties, is different from the surrounding land and can be assumed to be homogeneous in terms of its land properties. The degree of homogeneity or internal variation will vary depending on the scale and intensity set out in the evaluation objectives (FAO 1976).

Although the size of the land units should be kept as small as possible to limit generalization, too many units can become unmanageable as each individual land unit is considered individually as to its land properties and requirements. The decisions about the size, number and delineation of land units are often determined by data availability. Soil type boundaries are probably the most suitable delineations of land units for most land uses, but soil information is often unavailable at the required scales. In these cases other available datasets, such as terrain units (i.e. land components), can be used. Land components are sometimes used as land units in medium-scale studies (1:25 000 to 1:500 000) because many physical land properties, including soil, climate and biology, are related to terrain (MacMillan, Jones \& McNabb 2004; Speight 1977). Due to the unavailability of semi-detailed soil data in the Western Cape (see Section 5.2.1), land components were used as basic mapping units (land units) in this study.

This chapter outlines the procedures to establish a land unit database. In the first part of the chapter the techniques for delineating land components, in particular the ALCoM algorithm, are explained. The rest of the chapter focuses on populating the database with land property information.

\subsection{LAND COMPONENT MAPPING TECHNIQUES}

Land components are essentially subdivisions of landscapes and are frequently used in suitability analysis as a basic mapping unit (i.e. land unit). Although 'landscape' has been variously defined, it can be conceptualized in the terrain analysis context as a hierarchical collection of terrain forms comprising land regions, land systems, land forms, hillslopes, land components, and land elements. 
A land element is the smallest practical terrain unit at a given scale of mapping. McDonald et al. (1984) suggest that such elements should not be less than 150x150 metres in size (i.e. less than 2.25 hectares) at 1:50 000 scale, but can potentially be much larger in homogeneous landscapes. Land elements can be combined to form land components which are typically associated with ridge crests, fallfaces, midslopes, and footslopes (Argialas 1995; Dymond, Derose \& Harmsworth 1995).

Hillslopes (also called profiles) are sequences of land components orientated in the slope direction (see Figure 6-1). The sequences of components forming hillslopes differ according to number and type. Fallfaces are, for instance, not present on low hills while midslopes are absent on mesas. Complex hillslopes can include multiple occurrences of a particular type of component.

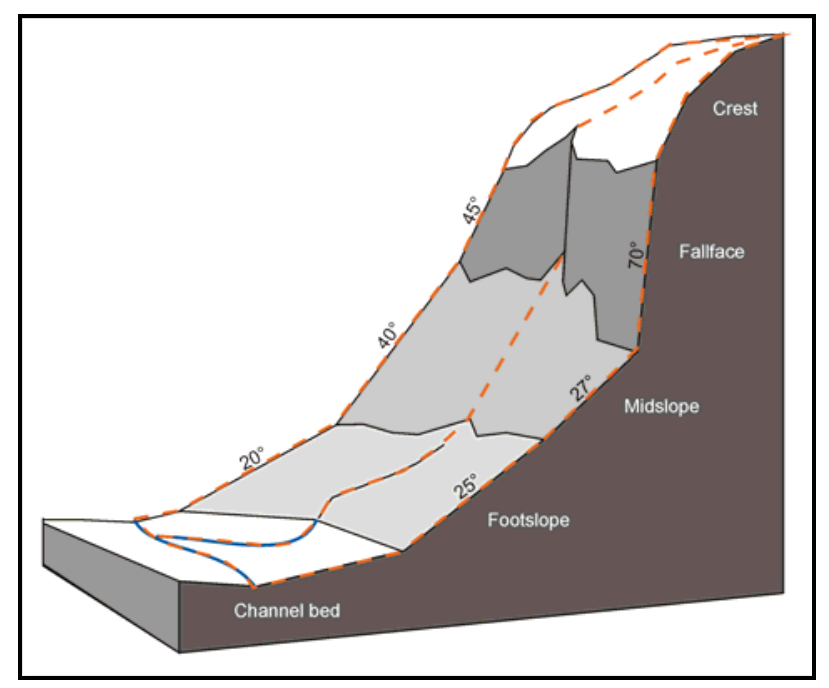

Source: Van Niekerk \& Schloms (2001)

Figure 6-1 Two hypothetical hillslopes, each consisting of a sequence of five land components

Landforms (e.g. hills, mesas, escarpments) are essentially sequences of hillslopes arranged perpendicular to the slope direction and they are, in many cases, the main focus of terrain analysis. However, landforms have little value in land suitability analysis because land properties can vary considerably within a landform. In the southern hemisphere, temperatures will, for instance, be considerably higher on north-facing hillslopes than on south-facing hillslopes, while soils will be deeper in channel beds than on crests. Land components are thus the most appropriate demarcations to use as the basis for suitability analysis.

Traditional techniques for demarcating land component boundaries are subjective and timeconsuming as they rely on the visual analysis of terrain data. The following sections overview terrain analysis, geomorphometry and the automated land component mapping techniques considered for demarcating land components in the Western Cape. 


\subsubsection{Terrain analysis}

Terrain analysis was defined in Section 4.1.3.4 as the study of the nature, origin, morphological history and composition of landforms, the result of which is a landform or land component map. Land components can be mapped by studying topographical maps, interpreting aerial photographs (Speight 1977) and making field measurements (Graff \& Usery 1993). Such terrain analysis techniques are considered to be an art without formal theory and often rely on the interpreter's implicit terrain-related knowledge of the area being studied (Irvin, Ventura \& Slater 1997). Such skill is the product of lengthy, expensive training and experience (Argialas 1995). The subjective nature of terrain analysis is a major drawback because in most cases it is impossible to make any useful comparisons between land component maps produced by different analysts or even by the same analyst at different times (Speight 1977). The interpretation and mapping of land components are extremely time-consuming, labour-intensive and expensive tasks (Adediran et al. 2004) and is difficult to verify in the field owing to the fractal nature of topography (Hengl, Gruber \& Shrestha 2004). Consequently, more objective and automated methods are needed to map land components. Computer analysis of geomorphometry is a convenient option.

\subsubsection{Geomorphometry}

Geomorphometry, the numerical representation of topography, combines mathematics, engineering and computer science. In the past, geomorphometry concentrated on the geometry of terrain, but technical advances in computing, analytical algorithms, input-output devices and large sets of topographic data have shifted the focus to digital representation of terrain, process modelling and generalization (Adediran et al. 2004).

Recently, the increasing availability of digital elevation models has promoted the use of computer technology for the calculation and discrimination of terrain properties. DEM-derived data sets such as slope, aspect, hydrographical pattern and shaded relief are being increasingly exploited in terrain analysis. These morphometric parameters are not only less prone to human error but can be used to objectively and quantitatively compare terrain units (Dymond, Derose \& Harmsworth 1995; Giles \& Franklin 1998).

GIS is often used to support geomorphometry and land component mapping. The most common approach is to use GIS overlaying techniques to combine DEM derivatives such as slope and aspect to create unique, homogeneous morphological units (Adediran et al. 2004). Classification is required to convert the continuous slope and aspect raster surfaces into regions (polygons). Once the slope and aspect rasters have been classified, they are usually converted to vector 
format and overlaid to create new polygons representing combinations of aspect and slope. The overlay operation is, in many cases, followed by a conflation operation to get rid of insignificantly small polygons.

The use of overlay techniques to delineate morphological land units is simple, fast and can be done with standard GIS software. The problem with this technique is the way in which terrain is generalized during the classification process. Slope aspect is usually classified into nine standard aspect classes representing north, north-east, east, south-east, south, south-west, west, northwest, and no aspect (level) (Dymond, Derose \& Harmsworth 1995), while slope gradient is usually classified into a number of equal-interval classes. The effect of applying such classification schemes over the entire extent of the slope gradient and aspect rasters (i.e. as a global raster operation) is that class breaks will not likely coincide with local terrain transitions. This is especially problematic for slope breaks because small transitions in slope gradient can have drastic effects on land properties such as soil and vegetation cover.

The hypothetical slopes shown in Figure 6-1 illustrate that slope breaks do not occur at consistent slope gradients. For instance, there is an abrupt $\left(33^{\circ}\right)$ transition between the midslope and the fallface, while in the far range the transition is less acute $\left(5^{\circ}\right)$. Although the slope gradient variance along slope breaks is exaggerated in the illustration, it demonstrates that land components such as midslopes can differ significantly in terms of slope gradient. While these subtle differences will be relatively easy to map using manual techniques (i.e. topographic map and aerial photo interpretation), it is unlikely that the boundaries of land components will be mapped along natural slope breaks using the global classification and overlay approach described above (Dymond, Derose \& Harmsworth 1995).

\subsubsection{Automated mapping}

The inability of GIS classification and overlaying techniques to accurately identify and map slope breaks prompted Dymond, Derose \& Harmsworth (1995) to develop an algorithm to automatically detect such transitions from a DEM. The algorithm, which was implemented in FORTRAN, starts by classifying aspect into eight $45^{\circ}$ classes and then splits each resulting aspect region into two major land components representing upper and lower slopes. Each aspect region is split along the five-metre vertical interval contour that is most likely to represent a slope break. The most appropriate division is determined by comparing the variances of each component pair created at consecutive elevation intervals. The split elevation giving the smallest average variance is used if the difference between the upper and lower slope angles is significant. If necessary, the process is repeated for each of the upper and lower land components, to 
potentially produce four land components representing upper-upper slopes, upper-lower slopes, lower-upper slopes and lower-lower slopes.

According to Dymond, Derose \& Harmsworth (1995), the algorithm produces results which compare well with results obtained from manual mappings using an analytical stereoplotter. However, some land component boundaries are not realistic because slope breaks often occur at varying elevations. Better results are obtainable by using distance from streams instead of elevation as the split lines. A major limitation of the algorithm is that a maximum of four land components can be mapped per aspect region, while in reality many more slope breaks occur. Small $(5 \mathrm{~m})$ elevation or distance intervals are also necessary to accurately delineate land component boundaries. This has a significant effect on the performance of the algorithm as several land component pairs must be examined regarding variability for each aspect region. For an aspect region with a 100m elevation range, 20 land component pairs have to be examined for the first division (upper and lower slopes). If a second division is necessary, the number of iterations doubles. This approach requires extremely intensive computer processing and is not viable for large areas like the Western Cape. Clearly, an improved, more efficient algorithm for automated land component mapping is needed.

\subsection{Automated component mapping with ALCoM}

Based on the work done by Dymond, Derose \& Harmsworth (1995) a new algorithm, called the Automated Land Component Mapper (ALCoM), was developed by Van Niekerk \& Schloms (2001) to automatically map land components from a DEM. ALCoM differs from Dymond, Derose \& Harmsworth's (1995) algorithm in that it relies on a statistical technique developed by Jenks (1967) to identify natural slope breaks. Although Jenks' (1967) technique can be applied to identify natural breaks within any data set, it proved to be very effective in identifying slope breaks when applied to slope gradient data (Van Niekerk \& Schloms 2001). The statistical detection of natural breaks is relatively fast and was expected to be more efficient and accurate than the sequential land component mapping approach of Dymond, Derose \& Harmsworth (1995).

Like the Dymond, Derose \& Harmsworth (1995) algorithm, ALCoM (see Figure 6-2) starts with the creation of an aspect raster (step 1), which is then classified (step 2) into nine $45^{\circ}$ aspect classes. The resulting aspect classification is regionalized in step 3, resulting in unique polygons representing individual aspect regions or directional hillslopes. The focus of the algorithm then changes from a global to the local level by calculating the slope gradient for each aspect region (steps 4 and 5). The most prominent slope break in an aspect region is then determined by employing Jenks' (1967) technique with the number of breaks set to one (step 6). 


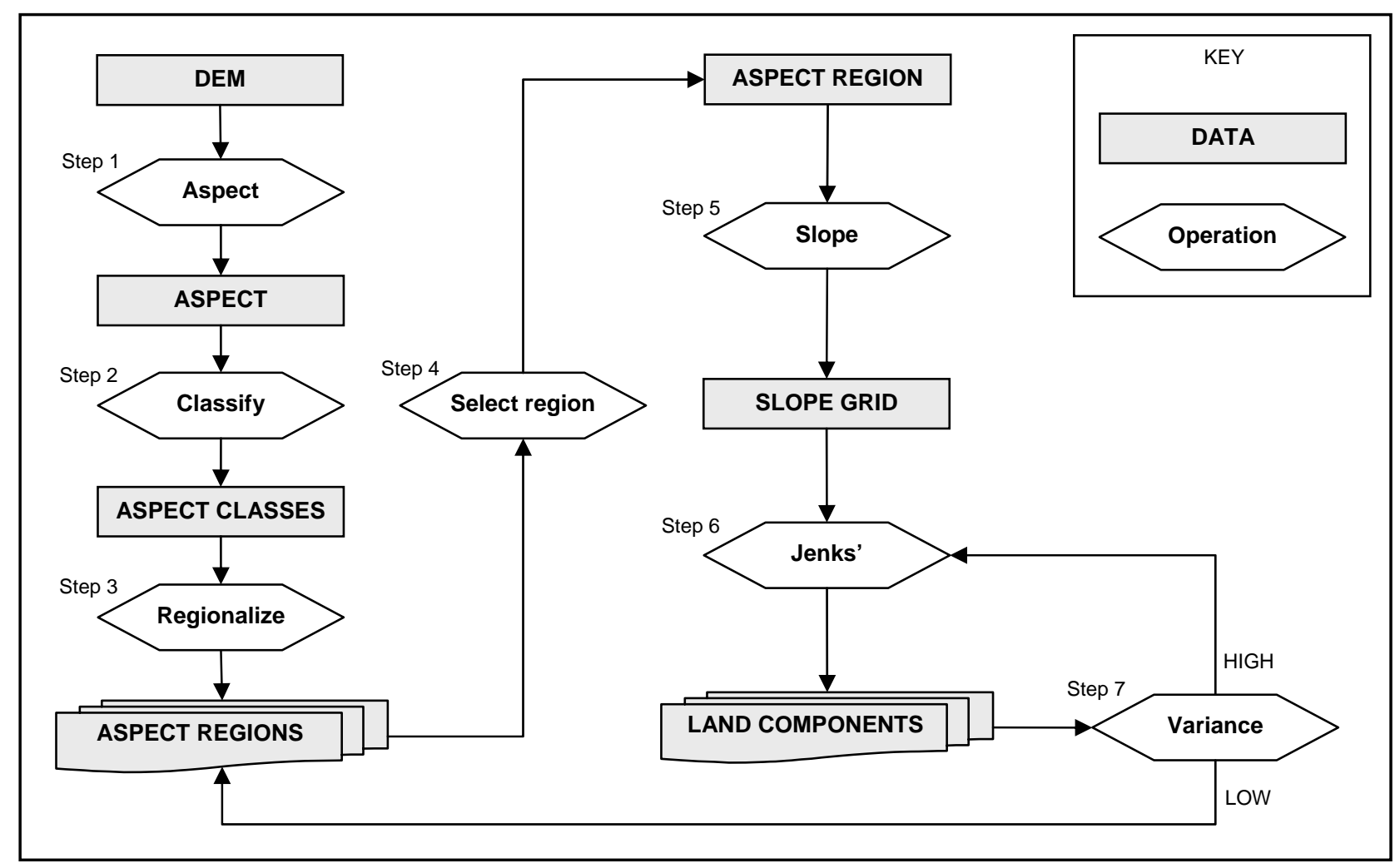

Figure 6-2 The ALCoM algorithm

In step 7, the slope gradient variance of each of the resulting land components is determined and the detection of slope breaks is repeated with increasing number of breaks until each of the resulting land components is homogeneous in terms of slope gradient. To determine homogeneity, the slope gradient variance (SGV) of each land component is compared to the $\mathrm{SGV}$ of the entire aspect region. A land component is considered to be homogeneous only if its $\mathrm{SGV}$ is ten times lower than the overall $\mathrm{SGV}$ of the aspect region. A lower ratio results in the mapping of smaller, more homogeneous land components, while a higher ratio produces larger, less homogeneous land components. Once the acceptable level of homogeneity is reached, the next aspect region is considered. The algorithm terminates when all land components for all aspect regions have been mapped.

ALCoM was automated in ArcView GIS using the Avenue programming language (ESRI 2002a) (see Appendix B for source code) and tested on a $15 \times 5 \mathrm{~km}$ area in the Stellenbosch region (see Figure 6-3). The test area was chosen because it includes a variety of land components ranging from predominantly level areas in the west to fallfaces, ridges and crests in the east (Figure 6-4). The researcher also has implicit knowledge of the area, which is essential for the interpretation of the results. 


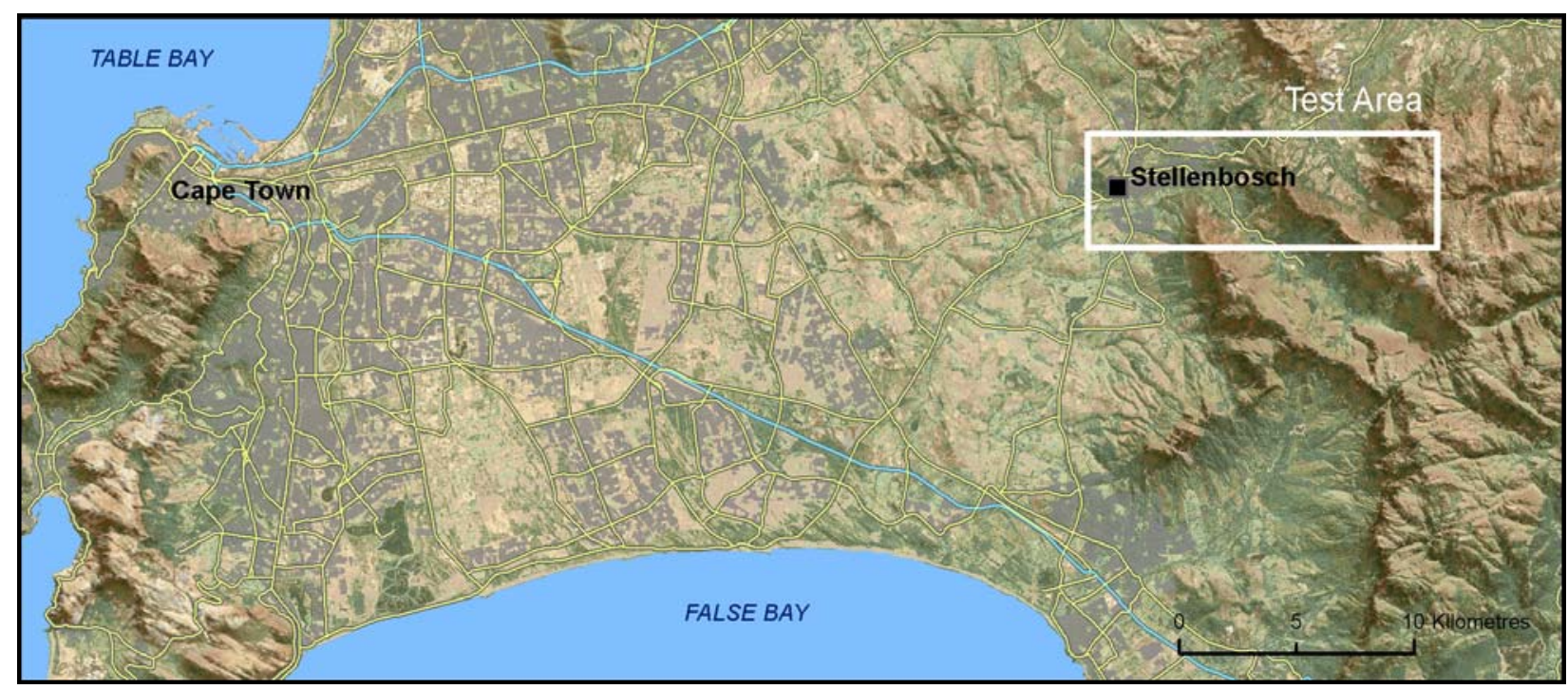

Figure 6-3 Location of the test area for ALCoM

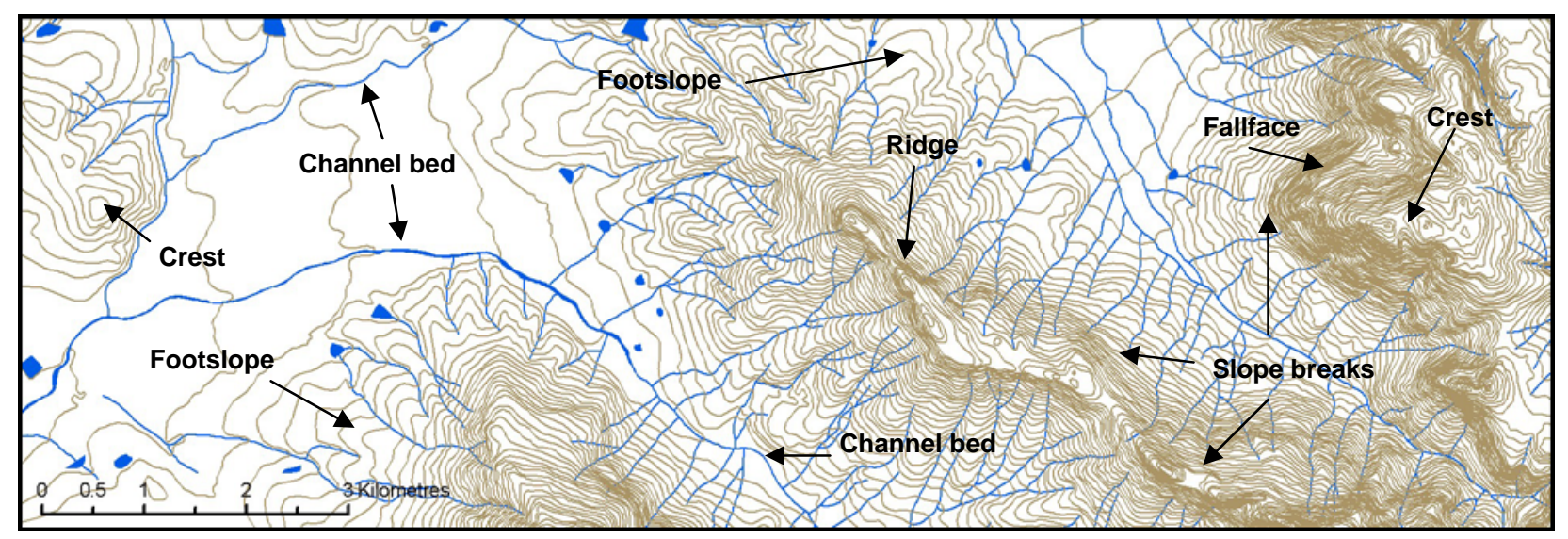

Figure 6-4 Detailed view of the test area, with selected terrain features indicated

ALCoM was applied to the WCDEM (see Section 5.1.2) and produced 1057 land components for the test area. The land components were visually compared with aerial photographs, orthophoto maps, topographical maps and SPOT 5 satellite images. The results (see Figure 6-5) show that ALCoM is very sensitive to small changes in relief, resulting in a highly detailed land component map.

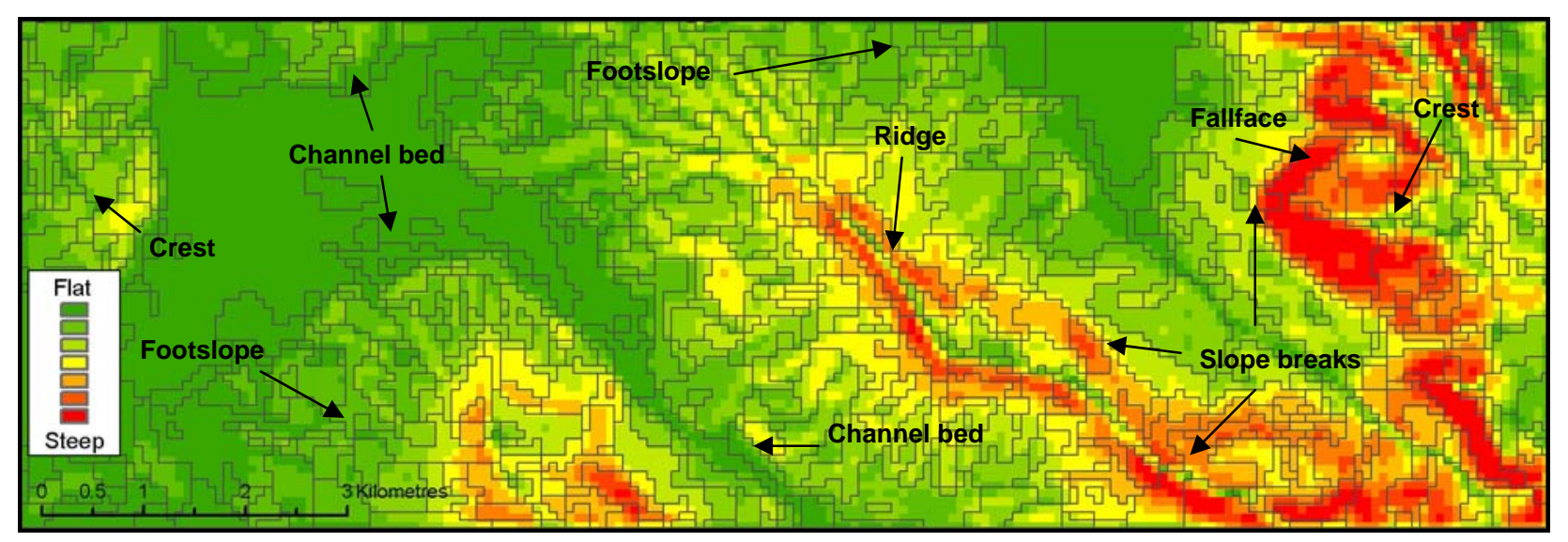

Figure 6-5 Land component boundaries mapped by ALCoM 
Although the high level of detail of the resulting land components makes comparison difficult, visual inspection shows that the boundaries of land components correspond very well with slope breaks. Slope breaks that do not follow contour lines or that are not parallel to streams are accurately mapped. This is an improvement over the Dymond, Derose \& Harmsworth (1995) algorithm. Statistically, the land components are relatively homogeneous with standard deviations of $2.9^{\circ}$ and $39.6^{\circ}$ for slope gradient and aspect respectively. The relatively high standard deviation of slope aspect is attributed to the nine $45^{\circ}$ classes that were used to derive aspect regions (see step 1 in Figure 6-2) and the larger range of possible values $\left(0-359^{\circ}\right)$.

The efficiency of ALCoM is considerably higher than that of the Dymond, Derose \& Harmsworth (1995) algorithm, as the number of iterations needed to detect slope breaks with $\mathrm{ALCoM}$ is not related to elevation difference. Because the maximum number of iterations per aspect region is equal to the number of slope breaks, no unnecessary iterations are required. In spite of the improvement in efficiency, however, ALCoM still requires considerable computer resources. Based on the 1.3 hours processing time that was required to generate the land components in the test area, it is estimated that more than 100 days of processing time would be needed to analyse the entire Western Cape province. Because such a long process is unlikely to complete successfully without interruption, a more efficient solution is required. Owing to its ability to efficiently analyse large data sets, image processing was considered as alternative for the automated demarcation of the Western Cape's land components.

\subsection{Image processing techniques}

Image processing involves the manipulation of digital imagery to enhance its quality, change its format or to extract various types of information. It encompasses a wide range of techniques for which specialized computer programs are needed. Because images are essentially multilayered or stacked rasters, image processing can be used to analyse terrain data. This section focuses on two image processing techniques used in land component mapping, namely image clustering and segmentation.

\subsubsection{Image clustering}

Image clustering is most commonly used to convert multiband imagery into regions of similar attributes. The best-known clustering technique is the iterative self-organizing data analysis technique algorithm (ISODATA) (Hall \& Khanna 1977) frequently used to cluster multiband satellite imagery into regions of similar spectral reflectances. The process starts with the specification of the number of classes (clusters) needed, followed by the assignment of arbitrary mean values to each class. Each raster cell is allocated to the closest class mean in the feature 
space, class means are recalculated and each pixel is again allocated to the new class means. This procedure is repeated several times until the class means stabilize (Gibson \& Power 2000). The resulting classes are regarded as unique spectral combinations and, in most cases, are combined and converted to information classes (i.e. land cover classes) (Campbell 2006).

Instead of using ISODATA on satellite imagery, Irvin, Ventura \& Slater (1997) employed the technique on terrain attributes such as elevation, slope gradient, slope aspect (solar radiation) and curvature to map land components. Although the approach produced relatively good results, the histograms of many of the clusters had multimodal distributions, indicating that the land components were not homogeneous. Clustering techniques such as ISODATA are based on global thresholds, which often lead to overclustering (i.e. producing units that are too small) and/or underclustering (merging regions that do not belong together) because local contrasts are not considered or are not consistently represented (Definiens Imaging 2004). Another problem with clustering techniques is that the analyst needs to specify the number of clusters that should be generated (Adediran et al. 2004). Because there is no way in which the optimal number of clusters can be known in advance, the only way to find an appropriate value is through experimentation and knowledge of the area under study (Irvin, Ventura \& Slater 1997). In addition, the optimal number of clusters depends on the landscape being studied, making it unsuitable for application in large, complex areas requiring considerable experimentation and operator interaction.

\subsubsection{Image segmentation}

Like clustering, image segmentation algorithms group pixels into spatial regions (segments) which meet predetermined criteria of homogeneity (Definiens Imaging 2004). The main difference between clustering and segmentation is the way in which the image is regionalized. The conceptual difference between clusters and segments is illustrated in Figure 6-6.
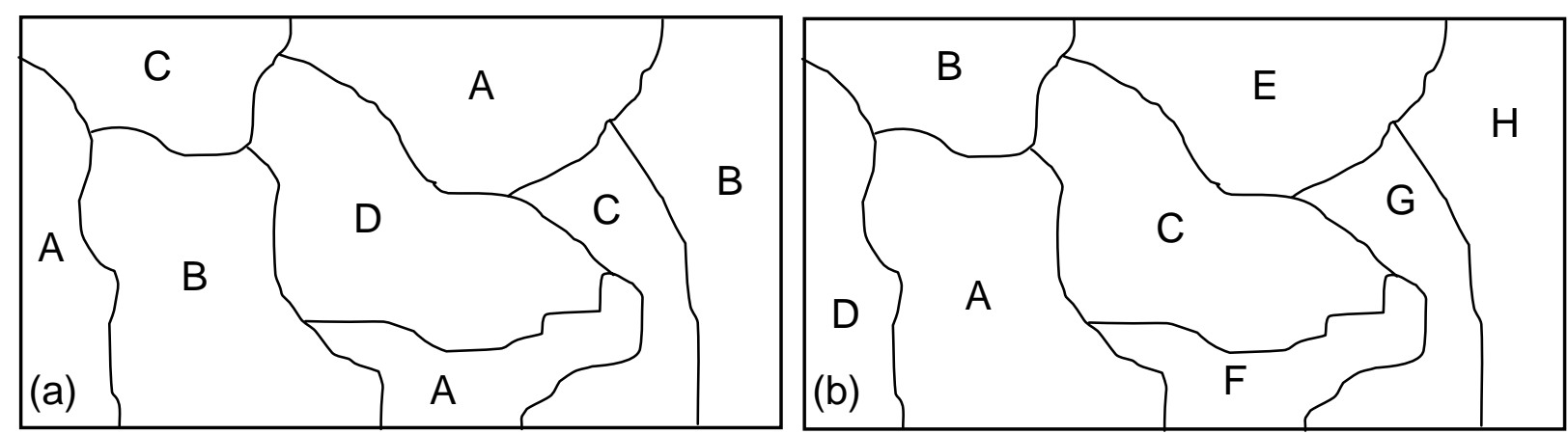

Figure 6-6 A conceptual comparison of clusters (a) and segments (b) in relation to attributes A to $\mathrm{H}$ 
Where clusters can consist of one or more groupings of pixels (polygons) that have similar attributes (indicated as A to $\mathrm{G}$ ) in the context of the entire image, segments are individual pixel groupings locally different from adjacent pixels.

Among the various existing image segmentation methods, region-growing segmentation is the best known. Region-growing segmentation clusters adjacent cells together if they have similar attributes. The segmentation process starts with a number of seed points that are either randomly sampled, statistically determined or specified by the user (Definiens Imaging 2004). The advantage of using randomly sampled seed points is that the procedure is autonomous and requires no input from the user. This approach can, however, lead to unpredictable results as the segmentation is highly sensitive to the initial positions of the seed cells. The use of random seeds also means the process cannot be repeated to produce the same segmentation result.

Better segmentation results can be obtained when seed points are statistically determined, but such measures are related to the global feature space of the image and are therefore relative to the specific image. Any change in the extent or position of the image will produce different seed points, which means that the segmentation will yield different results.

Seed points can also be specified by the analyst. Miliaresis (2001), for example, used cells that were pre-classified as ridges to discriminate mountainous and non-mountainous regions from a DEM. In another terrain application, Giles \& Franklin (1998) selected seeds based on field surveys and aerial photo interpretation to map land components. Campbell (2006) warns, however, that the use of training data imposes a structure on the clustering which might not match the natural clusters that exist in the data. In addition, the selection of training data can be a time-consuming, expensive and tedious undertaking, especially for large regions.

To overcome the limitations of region-growing image segmentation algorithms, De Kok, Schneider \& Ammer (1999) developed an algorithm to extract homogeneous image objects based on local contrasts. An important feature of this technique is that segmentation can be repeated to produce the same results, even if the extent or position of the image is changed. The so-called multi-resolution segmentation (MRS) technique can operate on multiple bands simultaneously and can produce multiscale segmentations on images having different resolutions. MRS was first implemented in 2000 as part of the eCognition object-orientated image analysis software package. eCognition is different from other remote sensing software in that image classification is not performed on individual pixels, but rather on segments (also called objects or polygons). The advantage of this approach is that important semantic information such as shape, texture and topology can be used. Such information is not apparent when considering individual pixels (Definiens Imaging 2004). 
The MRS algorithm is based on a pairwise region-merging technique which consecutively merges image cells. It involves an optimization procedure which, for a given number of objects, minimizes the average heterogeneity and maximizes their respective homogeneity. The procedure starts with single seed cells, which are iteratively merged into larger units while the upper threshold of homogeneity is not exceeded locally. If none of the neighbouring cells fall within the allowed thresholds, the best candidate becomes the seed and the merger process is repeated. This approach minimizes variability within merged cells (Definiens Imaging 2007).

The homogeneity threshold is indirectly set by the operator through a scale parameter which determines the number of segments that will be created. Conversely, variation within each segment increases as the scale parameter increases. Too high values could therefore lead to the loss of important detail, while too low values can result in an unnecessarily large number of small and almost identical segments. For efficient storage and faster processing, the number of segments should be kept to a minimum. The scale parameter is not a quantitative value and cannot be based on any scientific calculations. The only way to determine an appropriate scale factor is through experimentation (Definiens Imaging 2004).

Although no implementations of MRS for land component mapping from DEM could be found in the literature, the technique is expected to produce similar results to ALCoM due to the way in which it discriminates regions based on local contrasts. To compare MRS with ALCoM, the technique was applied to the same $10 \times 5 \mathrm{~km}$ test area shown in Figure 6-3. Slope and aspect rasters were derived from the WCDEM and imported as separate layers into the latest version of eCognition (version 7), named Definiens Developer (Definiens Imaging 2007).

To find an appropriate scale parameter, the rasters were segmented several times with increasing scale parameters. The resulting land components for each scale level were statistically compared with those produced by ALCoM. The comparison found that a scale parameter of 10 generated land components exhibiting similar levels of overall homogeneity of slope and aspect to those produced by ALCoM. While the land components produced by ALCoM are slightly more homogeneous (i.e. have a lower overall standard deviation) for slope gradient, slope aspect variance is slightly lower in the MRS data set (see Table 6-1).

Table 6-1 Statistical comparison of land components mapped using ALCoM and MRS

\begin{tabular}{|l|c|c|c|c|c|}
\hline LEVEL & \# of components & $\begin{array}{c}\text { Average size } \\
\text { (ha) }\end{array}$ & $\begin{array}{c}\text { OSD* of slope } \\
\text { gradient (deg) }\end{array}$ & $\begin{array}{c}\text { OSD* of slope } \\
\text { aspect (deg) }\end{array}$ & $\begin{array}{c}\text { OSD* of area } \\
\text { (ha) }\end{array}$ \\
\hline ALCoM & 892 & 8.4 & 2.9 & 36.7 & 25.6 \\
\hline MRS & 509 & 14.7 & 3.4 & 33.7 & 13.3 \\
\hline
\end{tabular}

* Overall standard deviation 
Overall, the MRS and ALCoM land components are very similar regarding slope and aspect variation. There is, however, a clear difference when the two data sets are compared visually (compare Figure 6-7 with Figure 6-5).

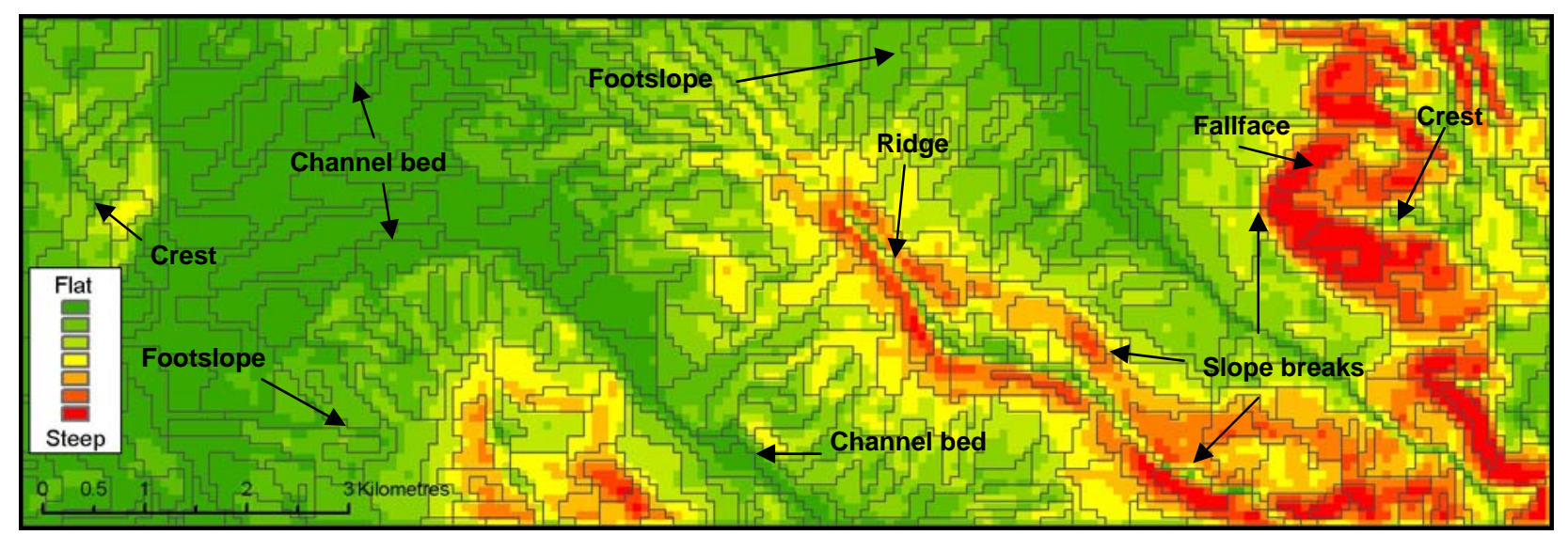

Figure 6-7 Land components mapped by multi-resolution segmentation

It is apparent that the land components in Figure 6-7 are more similar in size than those of ALCoM. This observation is supported by the fact that the standard deviation of land component area is 13.3 hectares for the MRS data set, while the corresponding value is 25.6 hectares for the ALCoM data set (Table 6-1). Although one would expect that areas with low relief will produce larger land components, the MRS algorithm is more sensitive to local terrain variability. In the ALCoM algorithm, local variability is related to the overall variability of a hillslope (i.e. aspect area), which can include large, relatively level and homogeneous regions that will not be subdivided into smaller land components.

ALCoM produces results that are more aligned with manual interpretations of terrain. However, for land suitability analysis, the land components produced by ALCoM are not necessarily better than those produced by MRS. The higher local sensitivity of MRS produces more detailed components in areas of moderate terrain which are more likely to be affected by land use changes. The ability of MRS to pick up subtle changes in moderate terrains is an invaluable asset for land evaluation purposes.

A major advantage of MRS for mapping land components is its efficiency. Where ALCoM took 1.3 hours to generate the land components in the test area, MRS completed the mapping in a fraction of a second! Definiens Developer is clearly an optimal solution for image processing compared to ALCoM which was developed under the constraints of an existing GIS environment (ArcView GIS). Based on the time it took Definiens Developer to process the test area, it was estimated that it would map the entire Western Cape in a matter of minutes. 


\subsection{Comparison of ALCoM and MRS}

Due to the indiscriminate way in which terrain is generalized, classification and overlay techniques are not suitable for delineating land components. A new technique, called the Automated Land Component Mapper (ALCoM), was developed to identify slope breaks to be used as boundaries for land components. Although the resulting land components are representative of the terrain features, the process proved to be too slow and unsuitable for implementation in large areas. A new image segmentation technique, called multi-resolution segmentation (MRS), was subsequently tested as an alternative to ALCoM. MRS relies on local contrasts and variability to produce segments (land components), irrespective of the extent and position of the input image. In comparison to ALCoM, MRS not only produces fewer and therefore more homogeneous land components, but completes the task in considerably less time. The results also show that MRS produces more detailed land components in areas of moderate terrain which are more likely to be targeted for land use changes. For land evaluation purposes, MRS is demonstrably the most appropriate method for mapping land components in the Western Cape.

\subsection{SEGMENTING THE WESTERN CAPE}

Multi-resolution image segmentation, as implemented in Definiens Developer software, was used to delineate land components/units from the Western Cape DEM (WCDEM). Because hardware and software constraints prevent it from being combined into one data set, the $20 \mathrm{~m}$ resolution WCDEM was obtained as 12 adjoining tiles covering the Western Cape. Although the high resolution of the WCDEM is an asset in that it ensures accuracy, the model is too large to be processed on a provincial level at its native resolution. To enable the combination of the data into a single data set, the individual blocks were rescaled to $80 \mathrm{~m}$-resolution and merged. A resolution of 80 metres was chosen because it is a factor of the original resolution, which meant that no changes in the extent of the data set was not required. A cell size of $80 \mathrm{x} 80 \mathrm{~m}$ is also considerably less than the required resolution (or minimum mapping unit) of 150m (see Section 5.1.1).

Although the volume of data of the rescaled version of the WCDEM (henceforth called WCDEM80) remains large, it can be analysed as a unit. However, as discussed in Section 5.1.1, resolution influences DEM quality and a reduction of resolution invariably reduces accuracy. To determine what effect the resolution change has on accuracy, the rescaled data set was assessed using the same method described in Section 5.1.3. The accuracy assessment shows that the WCDEM80 has a mean absolute error of nine metres compared to the seven metres of the 
WCDEM. The accuracy difference of two metres between the degraded and original data sets is insignificant, especially when compared to the $15 \mathrm{~m}$ vertical accuracy of the best alternative DEM (i.e. SRTM DEM).

Once merged, WCDEM80 was used to generate slope gradient and aspect rasters using ArcGIS 9.2. The resulting rasters were exported from ArcGIS as single-layer 8-bit TIFF images and combined in Definiens Developer. MRS was performed on these images, using a scale factor of 10, to produce 652704 land components covering the Western Cape. The average size of the land components (19.8ha) is considerably larger than the size of those produced by MRS in the test area (Table 6-1). This difference is attributable to the higher average slope of the test area $\left(14.4^{\circ}\right)$ compared to the entire Western Cape's $\left(6.5^{\circ}\right)$ because land components are larger in moderate terrain than in mountainous areas. The segmentation of the entire province performed well concerning slope and aspect homogeneity - the overall standard deviation of slope gradient and aspect being $2.9^{\circ}$ and $17.2^{\circ}$ respectively. This indicates that the land units produced for the whole Western Cape are more homogeneous than those mapped for the test area.

The segmentation procedure for the Western Cape was completed in less than one hour and the result was exported from Definiens Developer as an ESRI shape file used to facilitate the extraction of the environmental and physical land properties by a GIS.

\subsection{LAND PROPERTY EXTRACTION}

Once the soil, climate and terrain data was collected, it had to be prepared for importation into the land unit database. To do so, the land property data sets not already in ESRI grid format were converted and projected to conform to the land unit data. The universal transverse Mercator (UTM) map projection was chosen because the entire province is represented in a single zone (34S) and conforms to many other data sets of the Western Cape.

Once all the data sets accorded with the coordinate system, the extraction process involved calculating the average property value for each land unit. This procedure can be performed with the Zonal Statistics tool in ArcGIS Spatial Analyst extension. Unfortunately, the tool can only handle data sets of 100000 or fewer polygons and was therefore unable to calculate the land properties for the more than 650000 land units.

An alternative algorithm for extracting land properties was developed in ArcView GIS. Essentially, the algorithm breaks the task of calculating zonal statistics into areas of manageable size (quarter degrees) and repeats the process for each area consecutively. The process takes a few hours to complete. The Avenue code developed for this algorithm is attached as Appendix C. 


\subsection{STORAGE}

To determine the most efficient database solution, the land units were stored as vector polygons (areas) in both ESRI shape file format and in ESRI geodatabase format using ArcSDE and Oracle 9.1 RDBMS. Oracle is currently the most popular RDBMS and is widely considered to be the most robust DBMS software available (see Section 3.2.6). Because Oracle is essentially a non-spatial database, ArcSDE acted as a spatial database engine.

Shape files have become a de facto GIS data exchange format in South Africa and they are compatible with most spatial software packages. A polygon shape file is a simple, nontopological spatial database in which the geometrical location and shape of the polygons are stored separately from the attribute information. Each polygon can have multiple attributes which are stored as fields (columns) in a dBase table and each record (row) in the table corresponds to one polygon (ESRI 2007a).

The geodatabase and shape file land unit databases were compared as to their response times for queries and updates. Although the geodatabase was expected to be faster than the shape file format database, very little difference in response times was observed during querying. Updates to the attributes of the geodatabase were three times slower than updates to the shape file. The slower response times of the geodatabase are ascribable to the additional 'housekeeping' required by the versioning feature in geodatabases (ESRI 2007a). The major advantage of a geodatabase approach is that the size of the database is limited only by the available hardware. Theoretically, any number of polygons can be accommodated in a geodatabase, whereas shape files are limited to three billion polygons (ESRI 2002b). Because the number of land units was fewer than one million (652 704), the additional capacity of a geodatabase was not needed so that the land unit database will be stored in shape file format. Because of the loosely coupled design of the system, the land unit database can be migrated to Oracle when more storage space is required without affecting the other system components.

\subsection{SUMMARY}

The land unit data set is an efficient representation of land as it combines a range of terrain and other land variables into logically delineated polygons along with their attributes. Because CLUES uses land units as the basic analysis and mapping unit, special care had to be taken to ensure that land units represent homogeneous parcels of land. Several techniques were considered for the optimal delineation of land units, with ALCoM and image segmentation producing the best results. The latter technique is the more efficient one and was used to map the more than 600000 land units covering the Western Cape. Once the land units were delineated, it 
was used to extract soil, terrain and climatic properties from the collected data sets. This process was automated in ArcView.

The land unit database is the basis for land suitability analysis using CLUES as it represents the basic mapping units along with their land properties. To determine the suitability of each land unit for a particular land use, land use requirements in the form of rules must be defined. The land requirement rules are stored in a database separate from the land units. The development of this so-called knowledge base is discussed in the next chapter. 


\section{CHAPTER 7: DEVELOPING THE KNOWLEDGE BASE}

In the expert systems approach to land evaluation systems development, the knowledge base is a collection of land requirement rules based on existing expert knowledge. The rules are stored in predefined format to comply with the inference engine which carries out the suitability analysis. Most existing expert systems are single-user systems, meaning that a database of rules is created to explicitly carry out suitability analysis for a particular project or user. In multi-user applications such as CLUES, each user must be able to populate their own individual knowledge base with rules. A multi-user knowledge base is therefore required to store and manage the rules of each user. The design and implementation of a database is discussed in this chapter in two consecutive main sections. To ensure that the database includes all the necessary data to effectively support the suitability analysis procedure, a structured methodology called logical data modelling, was used in its development.

\subsection{LOGICAL DATA MODELLING METHODOLOGY}

The CLUES database was designed using the logical data modelling approach. Logical data modelling (LDM) is based on the philosophy that "business data [such as land use requirements] have an existence that is independent of how they are accessed, who accesses them, and whether or not such access is computerized" (Fleming \& Von Halle 1989:9). The methodology is entirely data-driven and is not biased by any application requirements or technological considerations. It also facilitates comprehensive understanding of the business information requirements and effective communication of these requirements to designers, developers and users. The wellstructured technique provides a foundation for the design of correct, consistent, sharable and flexible databases using any database technology and software. The process involves the following ten sequential design activities:

1. Identify major modelling entities.

2. Determine operational relationships between entities.

3. Identify primary keys.

4. Define foreign keys.

5. Determine key business rules.

6. Add remaining non-key attributes.

7. Normalize data structure.

8. Specify additional attribute business rules.

9. Combine user views.

10. Integrate the model with existing data models. 
Each of these ten sequential steps was completed during the operational design of the CLUES database as described in the following sections.

\subsubsection{Identify major modelling entities}

Entities are the key objects of interest to the user and can either be physical (e.g. user, land unit) or abstract (e.g. land requirement, land property) in nature. For the CLUES database, six objects were identified, namely users, land uses, land requirements, land requirement rules, land properties and land units. These objects are major entities. The user entity represents any user who has access to the system and land use is the object being evaluated. A land requirement is the third entity and, because requirements are sets of rules, each land requirement rule is considered to be a discrete entity. During suitability analysis, the land requirement rules are compared with land properties, which are related to the physical land units covering the study area. Two other objects, namely project and data source are added for operational reasons bringing the total number of objects to eight. The project entity was added as an abstract concept to represent a specific suitability analysis because it is expected that users will need to work on several different suitability analyses at any given time. The data source entity was added because it is envisaged that the system will eventually include hundreds of land properties and that metadata about each property will be needed to keep track of it.

\subsubsection{Determine operational relationships between entities}

The second step in logical data modelling is to determine relationships between entities. All relationships have direction, which can be defined as one-to-one (1:1), one-to-many (1:M), or many-to-many (M:M). A one-to-many relation between entities $\mathrm{A}$ and $\mathrm{B}$ signifies that one instance of A will relate to many instances of B. For example, one land use will relate to many land requirements.

The relationships and directions for each pair of the identified entities are specified in Table 7-1. The relationship matrix indicates that USER (first row) has 1:M relationships with LAND_USE (second column) and PROJECT (seventh column) as each user can specify many land uses and work on several projects. The notation 1:M indicates direction, which means that an inverse (i.e. M:1) relationship exists between, for instance, LAND_USE (second row) and USER (first column). There is a many-to-many relationship between PROJECT and LAND USE because, in optimal land use identification, each project can include many land uses and a particular land use can feature in several projects. 
Table 7-1 Relationship matrix of entities in the knowledge base

\begin{tabular}{|c|c|c|c|c|c|c|c|c|}
\hline ENTITIES & User & Land use & $\begin{array}{l}\text { Land } \\
\text { requirement }\end{array}$ & $\begin{array}{l}\text { Land } \\
\text { requirement } \\
\text { rule }\end{array}$ & $\begin{array}{l}\text { Land } \\
\text { property }\end{array}$ & Land unit & Project & Data source \\
\hline User & - & 1:M & * & * & * & * & 1:M & * \\
\hline Land use & $M: 1$ & - & 1:M & * & * & * & M:M & * \\
\hline $\begin{array}{l}\text { Land } \\
\text { requirement }\end{array}$ & * & $M: 1$ & - & 1:M & 1:1 & * & * & * \\
\hline $\begin{array}{l}\text { Land } \\
\text { requirement } \\
\text { rule }\end{array}$ & * & * & M:1 & - & * & * & * & * \\
\hline $\begin{array}{l}\text { Land } \\
\text { property }\end{array}$ & * & * & $1: 1$ & * & - & M:M & * & M:1 \\
\hline Land unit & * & * & * & * & M:M & - & * & * \\
\hline Project & $M: 1$ & M:M & * & * & * & * & - & * \\
\hline Data source & * & * & * & * & 1:M & * & * & - \\
\hline
\end{tabular}

* No direct relationship

Each land use relates to a number of land requirements and each land requirement is related to many land requirement rules. Although there is an indirect relationship between users and land requirement rules (i.e. via the entities land use and land requirement), only direct relationships are considered in the matrix. The relationship between land units and land use is also indirect.

In order to evaluate each land unit's suitability, there needs to be a 1:1 relationship between land requirement and land property. There is a many-to-many relationship between land unit and land properties, because each land unit will likely have many properties, while each property is represented by many land units. Concerning metadata, several land properties can have the same data source.

\subsubsection{Identify primary keys}

The third step in the LDM methodology is to add key attributes to each entity. An attribute is an atomic unit of data about an entity. The most important attribute of any entity is the primary key used to uniquely identify a specific occurrence of an entity. Because each of the entities is created and managed by the users, identification numbers are used as the primary key for each 
entity. Each primary key is named using the entity name and the suffix '_ID'. For instance, PROJECT_ID is used as the PROJECT entity's primary key. The underscore character is used instead of spaces as most DBMS do not allow spaces in attribute names. Upper-case characters are used for uniformity. The same convention is followed for the entity names (i.e. land use is changed to LAND_USE)

\subsubsection{Define foreign keys}

The definition of foreign keys involves the identification of keys that relate to other entities. Due to the simplicity of the data model, all of the foreign keys for the relationships in the matrix are defined to be the primary keys of the related entities. For instance, the USER entity is configured to relate to the LAND_USE entity through the USER_ID attribute, while the LAND_USE_ID is used to relate to LAND_REQUIREMENT.

\subsubsection{Determine key business rules}

Key business rules govern the effects of insert, delete and update operations on relationships and they address the integrity of attributes through placing constraints on the values of attributes (i.e. they impose domain integrity). Insertion implies that all the relationships in Table 7-1 are defined as being 'dependent' on one another. In other words, a new instance of a child entity (e.g. LAND_USE) can only be created if an instance of the parent entity (e.g. USER) already exists. Conversely, for the deletion of instances, parents can only be deleted if no child instances occur. To ensure the integrity of the entities, primary keys are not allowed to be null (i.e. zero or empty), must always be a number and may never be duplicated (i.e. two records may not have the same value).

\subsubsection{Add remaining non-key attributes}

The sixth step of the LDM approach is to add non-key (i.e. not primary or secondary key) attributes to each entity. The result of this activity is shown in Table 7-2. The USER_ID attribute in entity USER is the most important as it not only acts as the primary key for the entity, but also serves as foreign key to several other entities. In addition to the USER_ID, three other (non-key) attributes were added to the USER entity. The NAME attribute contains the name of the user and is simply a way of identifying who each user is. The PASSWORD item is needed to restrict access to the system and protect the users' projects. Email addresses are used to communicate with users about system updates, maintenance and password change notifications. 
Table 7-2 Entity attributes in the knowledge base

\begin{tabular}{|c|c|c|}
\hline ENTITY & ATTRIBUTES & TYPE \\
\hline USER & $\begin{array}{l}\text { USER_ID } \\
\text { NAME } \\
\text { PASSWORD } \\
\text { EMAIL }\end{array}$ & $\begin{array}{l}\text { Primary key + Foreign key } \\
\text { Non-key } \\
\text { Non-key } \\
\text { Non-key }\end{array}$ \\
\hline LAND_USE & $\begin{array}{l}\text { LAND_USE_ID } \\
\text { USER_ID } \\
\text { NAME }\end{array}$ & $\begin{array}{l}\text { Primary key } \\
\text { Foreign key } \\
\text { Non-key }\end{array}$ \\
\hline LAND_REQUIREMENT & $\begin{array}{l}\text { LAND_REQUIREMENT_ID } \\
\text { LAND_USE_ID } \\
\text { LAND_PROPERTY_ID } \\
\text { USER_ID } \\
\text { WEIGHT }\end{array}$ & $\begin{array}{l}\text { Primary key } \\
\text { Foreign key } \\
\text { Foreign key } \\
\text { Foreign key } \\
\text { Non-key }\end{array}$ \\
\hline LAND_REQUIREMENT_RULE & $\begin{array}{l}\text { LAND_REQUIREMENT_RULE_ID } \\
\text { LAND_REQUIREMENT_ID } \\
\text { SUITABILITY } \\
\text { LOWER_VALUE } \\
\text { MIDDLE_VALUE } \\
\text { UPPER_VALUE } \\
\text { CURVE_ID }\end{array}$ & $\begin{array}{l}\text { Primary key } \\
\text { Foreign key } \\
\text { Non-key } \\
\text { Non-key } \\
\text { Non-key } \\
\text { Non-key } \\
\text { Non-key }\end{array}$ \\
\hline LAND_PROPERTY & $\begin{array}{l}\text { LAND_PROPERTY_ID } \\
\text { DATA_SOURCE_ID } \\
\text { NAME } \\
\text { UNIT } \\
\text { MIN } \\
\text { MAX }\end{array}$ & $\begin{array}{l}\text { Primary key } \\
\text { Foreign key } \\
\text { Non-key } \\
\text { Non-key } \\
\text { Non-key } \\
\text { Non-key }\end{array}$ \\
\hline LAND_UNIT & $\begin{array}{l}\text { LAND_UNIT_ID } \\
\text { LAND_PROPERTY_ID } \\
\text { VALUE }\end{array}$ & $\begin{array}{l}\text { Primary key } \\
\text { Foreign key } \\
\text { Non-key }\end{array}$ \\
\hline PROJECT & $\begin{array}{l}\text { PROJECT_ID } \\
\text { USER_ID } \\
\text { LAND_USE_ID } \\
\text { NAME } \\
\text { MODIFIED } \\
\text { FUNCTION } \\
\text { MIN_X } \\
\text { MAX_X } \\
\text { MIN_Y } \\
\text { MAX_Y }\end{array}$ & $\begin{array}{l}\text { Primary key } \\
\text { Foreign key } \\
\text { Foreign key } \\
\text { Non-key } \\
\text { Non-key } \\
\text { Non-key } \\
\text { Non-key } \\
\text { Non-key } \\
\text { Non-key } \\
\text { Non-key }\end{array}$ \\
\hline DATA_SOURCE & $\begin{array}{l}\text { DATA_SOURCE_ID } \\
\text { NAME } \\
\text { SCALE } \\
\text { ORIGIN }\end{array}$ & $\begin{array}{l}\text { Primary key } \\
\text { Non-key } \\
\text { Non-key } \\
\text { Non-key }\end{array}$ \\
\hline
\end{tabular}


The only non-key attribute added to the LAND_USE entity is the NAME attribute which enables users to provide a description of each land use. For the LAND_ REQUIREMENT entity, a WEIGHT field defines the relative importance of a specific land requirement for a particular land use.

Five additional attributes were allocated to the LAND_REQUIREMENT_RULE entity. The SUITABILIY field accommodates the suitability level (i.e. N2, N1, S3, S2, S1) for each rule, while LOWER_VALUE, MIDDLE_VALUE and UPPER_VALUE specify the thresholds and central value for each rule. The CURVE_ID attribute differentiates between Boolean and fuzzy rules.

Four non-key fields were added to the LAND PROPERTY table to keep track of the different land properties for each land unit. While the NAME field stores a description of each land property, the UNIT field stores the unit (e.g. degree, metres and millimetres) used to quantitatively measure each property. MIN and MAX fields indicate the minimum and maximum values of each property respectively - values needed to automatically scale 'openended' rules (see Section 2.4.1).

In contrast to the LAND_UNIT entity which is allocated only one additional non-key attribute (VALUE) to store the individual land property values for each land unit, the PROJECT entity is supplemented with seven non-key attributes. The PROJECT entity is also provided with a NAME field for description purposes. An additional descriptive attribute, called MODIFIED, is included to store the date and time of a project's last updating. The rest of the attributes in the PROJECT entity are related to operational information. The FUNCTION field indicates the type of previous analysis conducted (i.e. land use suitability or optimal land use identification), while the MIN_X, MAX_X, MIN_Y and MAX_Y fields define the extent of the study area.

The final three non-key attributes added to the logical data model make provision for the description of each land property data source. To do so, metadata items NAME (description of the source), SCALE (map scale) and ORIGIN (original owner/developer) are included.

\subsubsection{Normalize data structure}

Normalization ensures internal consistency, minimal redundancy and maximum stability of data, without the loss of information. It is a method by which a logical data model can be optimized through three simple steps, namely:

1. Remove repeating or multivalued attributes to a separate child entity.

2. Remove all non-key attributes that are not dependent on the primary key.

3. Remove attributes that depend on other non-key attributes. 
Because no repeating or multivalued attributes were present and no attributes were found to be independent of the respective primary keys, the CLUES logical data model was already in normalized form and no further action was needed.

\subsubsection{Specify additional attribute business rules}

After normalization, the next step completed was to supplement the business rules - specified in Section 7.1.5 for key attributes - with additional business rules. The main activity during this stage of the logical data modelling process is to define the domains (i.e data type, length, range, uniqueness and null support) and triggers (i.e. insert, modify, and delete rules) for each attribute.

Table 7-3 shows the business rules for the USER entity. The business rules for the primary key USER_ID, defined in Section 7.1.5, are documented as being numeric. Because it is a primary key, it must be unique and non-null (i.e. no null values are allowed). Inserts of new instances of USER_ID are allowed and no triggers are necessary in such cases. The USER_ID cannot be updated or deleted without ensuring that no child entities are present. Update and delete triggers are needed for the USER_ID field.

Table 7-3 Business rules for USER entity in the knowledge base

\begin{tabular}{|c|c|}
\hline ATTRIBUTE & DOMAIN AND TRIGGER RULES \\
\hline USER_ID & $\begin{array}{l}\text { Data type: number } \\
\text { Format: integer } \\
\text { Uniqueness: unique } \\
\text { Null support: non-null } \\
\text { Insert trigger: none } \\
\text { Update trigger: not allowed } \\
\text { Delete trigger: ensure no child entities are present }\end{array}$ \\
\hline NAME & $\begin{array}{l}\text { Data type: text } \\
\text { Length: } 100 \\
\text { Uniqueness: non-unique } \\
\text { Null support: non-null }\end{array}$ \\
\hline PASSWORD & $\begin{array}{l}\text { Data type: text } \\
\text { Length: } 10 \\
\text { Uniqueness: non-unique } \\
\text { Null support: non-null }\end{array}$ \\
\hline EMAIL & $\begin{array}{l}\text { Data type: text } \\
\text { Length: } 50 \\
\text { Uniqueness: non-unique } \\
\text { Null support: non-null }\end{array}$ \\
\hline
\end{tabular}


The NAME field stores text and can accommodate names of up to 100 characters in length. To enable different users with the same name to use the system, uniqueness is set to non-unique (i.e. names can be duplicated). All users must supply a name (i.e. no null support).

The rules for the PASSWORD and EMAIL attributes are configured to be similar to those of the NAME field. The only difference is the width of the records, which was limited to 10 characters for PASSWORD and 50 characters for EMAIL. No triggers are needed for the NAME, PASSWORD and EMAIL fields as they are dependent on the triggers defined on the primary key (USER_ID). The domain and trigger rules for entities LAND_USE, LAND_REQUIREMENT, LAND_REQUIREMENT_RULE, LAND_PROPERTY, LAND_UNIT and PROJECT are provided in Appendix D.

\subsubsection{Combine user views}

The ninth step in the logical data modelling methodology is the integration of user views to eliminate redundancy and inconsistencies across views. Because each entity can be regarded as a view, the main task during this phase of the design process is to combine entities belonging together. In most cases, redundant entities have the same primary keys or have supertypesubtype relationships. Since no such entities are present in the CLUES logical data model, no further action was needed.

\subsubsection{Integrate with existing data models}

The logical data modelling methodology concludes with the integration of the designed model with existing models. Often one database needs to be integrated with other databases, a process which may require modifications to the logical data structure. Due to the spatial nature of land units, the land unit entity was replaced by a separate spatial database containing the geometrical and environmental properties of each land unit.

To perform a land suitability analysis, the items (i.e. columns) of this so-called land unit database are related to the records (i.e. rows) of the LAND_PROPERTY entity through the LAND_PROPERTY_ID. To effect this relationship, each item name in the land unit database that stores a land property was modified to reflect the LAND_PROPERTY_ID. Since item names cannot start with a number, a ' $\mathrm{P}$ ' prefix was used to differentiate land properties from other items. For instance, if the LAND_PROPERTY_ID for effective soil depth is 8, the corresponding item in the land unit database was renamed to 'P8'.

Once all the entities, keys, attributes and business rules had been defined, a logical data model diagram (LDMD) was created (see Figure 7-1). A LDMD is a pictorial representation of the 


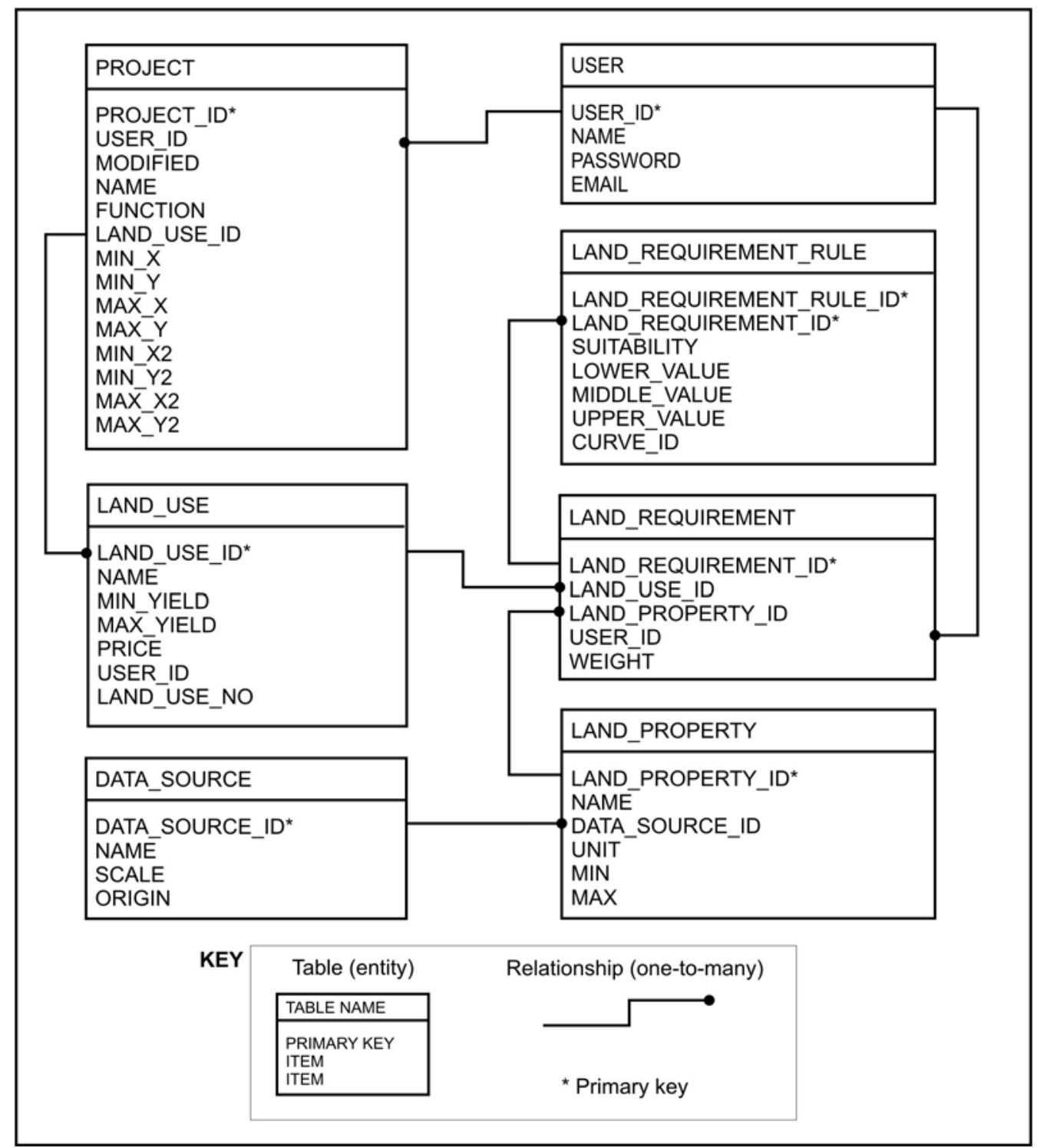

Figure 7-1 Logical data model diagram of CLUES knowledge base

logical data model that clarifies information relationships and enhances communication (Fleming \& Von Halle 1989). It documents the results of all the previous decisions (steps 1-9) in a single diagram.

\subsection{IMPLEMENTATION}

The CLUES database was implemented in the Microsoft Access database management system (DBMS). Microsoft Access was chosen for its simplicity and because of its user-friendliness. The software is often used for small Internet databases because they are easy to set up and are highly portable.

The translation of a logical data structure to a database involves the following four steps (Fleming \& Von Halle 1989):

1. Identify tables.

2. Identity columns. 
3. Adapt data structure to the product environment.

4. Implement entity, relationship and attribute business rules.

The implementation posed few challenges as the main activities involved the creation of the tables, adding the fields and setting the domain integrity rules. Most of the domain integrity rules were related to setting appropriate data types (e.g. numerical, text and memo) for each field. Although it is not expected that the user database will grow beyond a few megabytes, the data and structure can easily be exported to a more robust DBMS, such as Microsoft SQL Server or Oracle, should it be necessary. It was not necessary to implement queries or views in Microsoft Access as interaction with the database is managed through the graphical user interface, inference engine and web map service. The design and implementation of these website elements are discussed in the next chapter. 


\section{CHAPTER 8: DEVELOPMENT OF THE CLUES WEBSITE}

A website is a location connected to the Internet that maintains one or more web pages (Oxford English Dictionary 2008). It is a collection of web pages and related images, videos and other digital media hosted on one or more web servers. This standard definition fits the CLUES website which consists of a number of web pages, but CLUES differs from most websites owing to its ability to dynamically (i.e. on request) produce maps, making it a web mapping application, which is a special type of website. CLUES also includes operations related to database management and spatial analysis. The combination of all these functions necessitated a unique approach to its design and development as an application.

As discussed in Section 4.2, the website component of CLUES comprises three elements, namely the web map service (WMS), inference engine, and the graphical user interface (GUI). In combination, these components not only contain the full functionality of CLUES but also act as an interface between the two supporting databases described in the previous two chapters. This chapter details the development and content of each of the CLUES website's three elements, starting with the inference engine.

\subsection{THE INFERENCE ENGINE}

The inference engine is the key element in the CLUES website as it performs the essential function of calculating a land use suitability value for each land unit using the land requirement rules in the knowledge base. The resulting suitability values are stored in a temporary field in the land unit database, which is then used by the WMS to produce a suitability map. The following two subsections outline the methodology of how the inference engine algorithm was developed to calculate suitability and how it was implemented in CLUES.

\subsubsection{Suitability calculation procedure}

The procedure for calculating suitability values is encapsulated in Equation 8-1. Essentially, a land unit's overall suitability $(S)$ is determined by summarizing the product of each individual land use requirement weighting (importance value) and suitability value.

$$
S_{j}=\sum w_{i} s_{i}
$$

Equation 8-1

$S_{j} \quad$ is the overall suitability value for land unit $j ;$
$w_{i} \quad$ is the weight of land use requirement $i$; and
$s_{i} \quad$ is the suitability value of land property $i$.


As set out in Section 2.4.1, each land use requirement consists of multiple rules and each rule is related to a specific suitability level $(\mathrm{S} 1, \mathrm{~S} 2, \mathrm{~S} 3, \mathrm{~N} 1, \mathrm{~N} 2)$. To incorporate the suitability level into the suitability value calculation, a suitability level factor of 1 to 5 was introduced (see Table $8-1)$.

Table 8-1 Suitability level factors

\begin{tabular}{|c|c|c|}
\hline LEVEL CODE & LEVEL DESCRIPTION & RANGE \\
\hline S1 & Highly suitable & $>2.5 S_{j} \leq 3.5$ \\
\hline S2 & Moderately suitable & $>1.5 S_{j} \leq 2.5$ \\
\hline S3 & Marginally suitable & $>0.5 S_{j} \leq 1.5$ \\
\hline N1 & Unsuitable at present & $>0 S_{j} \leq 0.5$ \\
\hline N2 & Permanently unsuitable & $<0$ \\
\hline
\end{tabular}

These factors are multiplied by the membership values of each rule using Equation 8-2, the product being a suitability value for each land use requirement.

$$
s_{i}=\sum l_{k} y_{k}
$$

Equation 8-2

where $\quad s_{i} \quad$ is the suitability value for land requirement $i$;

$l_{k} \quad$ is the suitability level factor of rule $k$; and

$y_{k} \quad$ is the membership value of rule $k$.

\subsubsection{Membership value calculation}

The membership value $\left(y_{k}\right)$ of a rule is defined by a function of the lower $(\alpha)$, central $(\beta)$ and upper $(\gamma)$ values specified for each rule (see Section 2.4.2). Because a rule can be either symmetrical or asymmetrical, each rule needs to be deconstructed into one or two membership functions that can be used to calculate a rule's membership value. The steps taken to do so are discussed below.

\subsubsection{Represent rules as linear functions}

The first step in determining a methodology for calculating membership values was to represent rules as linear functions. Although a curved function, such as the s-function, is often preferred in fuzzy classifications, they are difficult to understand and are quite likely to be applied incorrectly by users. Linear functions are less complex and easy to implement and visualize. Figure 8-1 illustrates the effect of using linear functions for the Boolean and fuzzy rules defined in Table 2-4. Compared to Figure 2-11 in which the same rules were implemented using the s-function, it 
is clear from Figure 8-1 that the linear function produces a very similar classification and that the differences in the resulting membership values between these two fuzzy sets are insignificant.

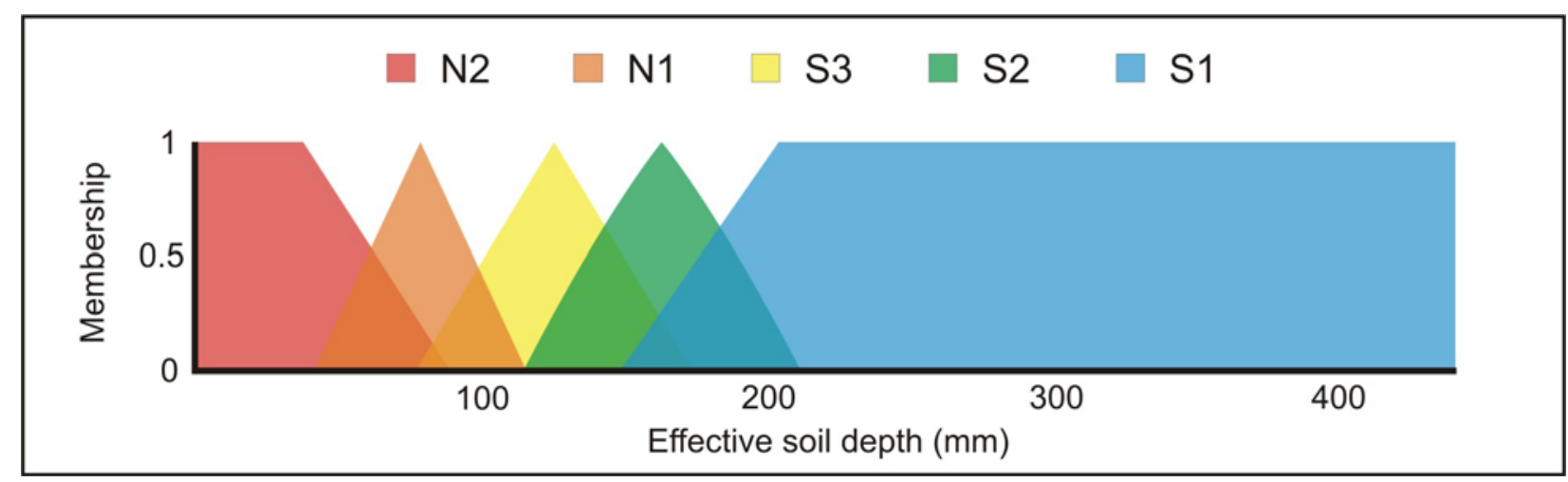

Figure 8-1 Levels of suitability of effective soil depths for perennial crops using linear fuzzy classification

For computational purposes, membership values for each rule are calculated by deconstructing each rule into one or more lines. Figure 8-2a illustrates how a symmetrical fuzzy rule can be defined using two lines, A and B. In this example, the lower value $(\alpha)$, central value $(\beta)$, and upper value $(\gamma)$ were set equal to 1,4 and 7 respectively on a range of $0-10$.
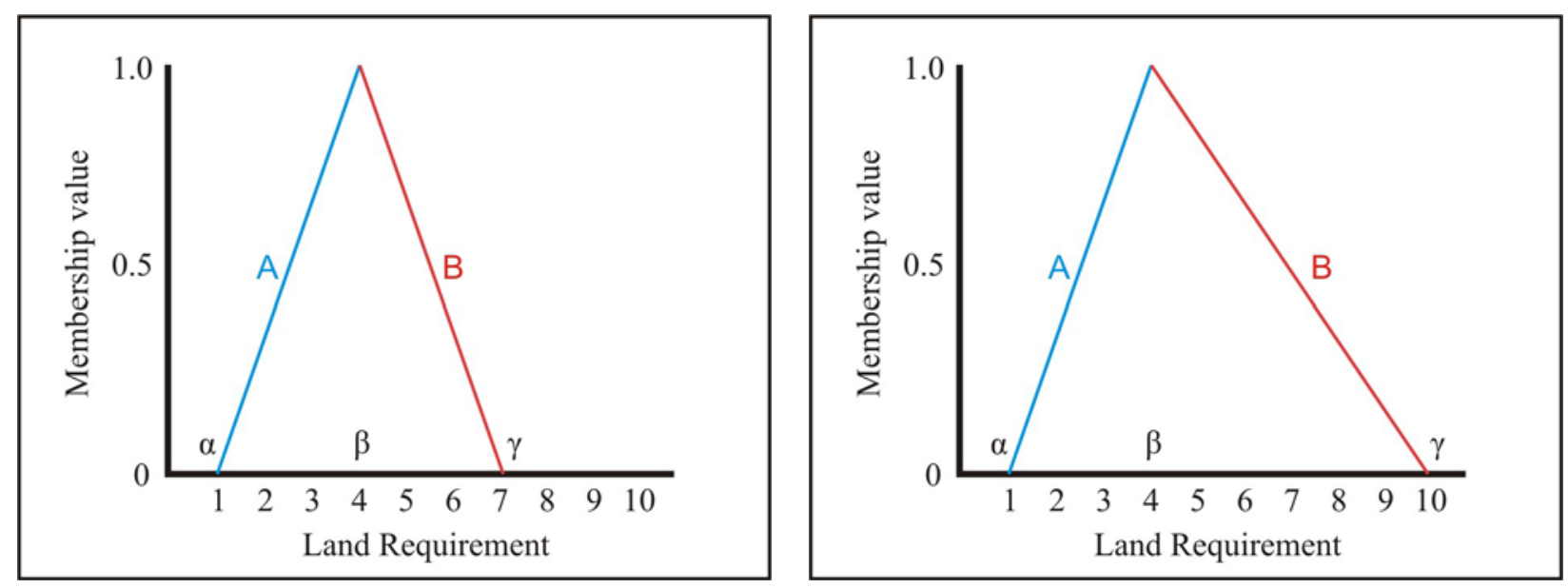

Figure 8-2 Symmetrical (a) and asymmetrical (b) fuzzy rules deconstructed to two lines, A and B.

The use of two separate line functions for each rule allows asymmetrical functions to be defined by specifying the central value to be off-centre. Such a rule is illustrated in Figure 8-2b, the lower $(\alpha)$, central $(\beta)$, and upper $(\gamma)$ values being set to 1,4 and 10 respectively. For rules where $\beta=\alpha$ or $\beta=\gamma$ (such as rule 6 in Table 2-4), only one line is needed to represent a rule.

\subsubsection{Determine membership function equations}

Because rules are defined as linear functions, membership values can be calculated using the yaxis formula for a line (Equation 8-3). In this formula, the value of $x$ is the land property value of a land unit, while $y$ represents the membership function. 
where $\quad y \quad$ is the $y$ coordinate value for a point on a line;

$m \quad$ is slope of the line;

$x \quad$ is the $x$ coordinate value for a point on a line; and

$b \quad$ is the $y$-axis intercept.

To solve Equation 8-3, values for $m$ and $b$ are required. Because the values for $\alpha, \beta$, and $\gamma$ and their corresponding $y$ coordinates (i.e. membership values) are known, these can be substituted into Equation 8-3 to calculate $b$. By setting $x=\beta$ and $y=1$ into Equation 8-3 the y-axis intercept is:

$$
b=1-m \beta
$$

Equation 8-4

From Equations 8-3 and 8-4 it follows that

$$
\begin{aligned}
y & =m x+1-m \beta \\
& =m x-m \beta+1 \\
& =m(x-\beta)+1
\end{aligned}
$$

Equation 8-5

The remaining variable to solve in Equation 8-3 is $m$, which can be calculated using Equation 8-6.

$$
m=\frac{y_{i}-y_{j}}{x_{i}-x_{j}}
$$

where $\quad m \quad$ is the slope of the line;

$y_{i}$ and $y_{j} \quad$ are the $y$ coordinates of any two points $i$ and $j$ on the line; and $x_{i}$ and $x_{j} \quad$ are the $x$ coordinates of any two points $i$ and $j$ on the line.

A positive slope indicates that the value of $y$ increases as $x$ increases (i.e. an ascending function), while a negative slope indicates that $\mathrm{y}$ decreases as $\mathrm{x}$ increases (i.e. a descending function). Functions for line A and line B in Figure 8-2a can therefore be regarded as being positive and negative respectively. Because the $\mathrm{y}$ values for $\alpha, \beta$, and $\gamma$ are known, they can be introduced into Equation 8-6 to calculate the slopes of lines $\mathrm{A}$ and $\mathrm{B}$ using Equations 8-7 and 8-8 respectively.

$$
m_{A}=\frac{1}{\beta-\alpha}
$$

$$
x \in[\alpha, \beta]
$$

Equation 8-7

where $\quad m_{A} \quad$ is the slope of the line A; 
$\alpha \quad$ is the lower limit of the fuzzy function; and

$\beta \quad$ is the central value of the fuzzy function.

$m_{B}=-\frac{1}{\gamma-\beta}$

$x \in[\beta, \gamma]$

Equation 8-8

where

$m_{B} \quad$ is the slope of the line B;

$\gamma \quad$ is the upper limit of the fuzzy function; and

$\beta \quad$ is the central value of the fuzzy function.

By substituting Equation 8-7 into Equation 8-5 it follows that, for line A, membership values can be calculated using Equation 8-9.

$$
y_{A}(x ; \alpha, \beta, \gamma)=\frac{x-\beta}{\beta-\alpha}+1 \quad x \in[\alpha, \beta] \quad \text { Equation 8-9 }
$$

Similarly, Equation 8-8 can be substituted into Equation 8-5 to produce Equation 8-10, which can be used to calculate membership values for line B.

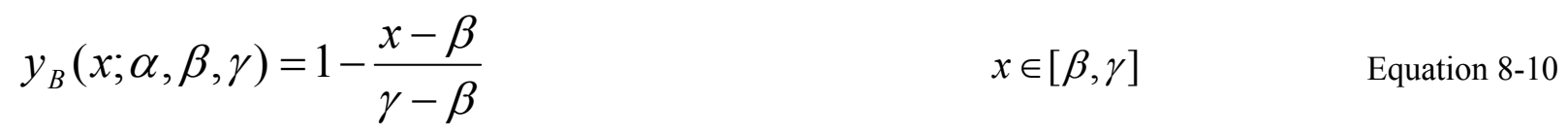

Linear functions can also be used to represent Boolean rules by setting the y-axis intercept $(b)$ equal to 1 and the slope $(m)$ equal to 0 in Equation 8-3. By doing so, Equation 8-3 is reduced to:

$$
\begin{array}{ll}
y_{C}=1 \quad x \in[\alpha, \gamma] \quad \text { Equation 8-11 }
\end{array}
$$

where $\quad y_{C} \quad$ is the membership value for Boolean rules.

\subsubsection{Suitability value calculation}

The application of Equations 8-9, 8-10 or 8-11 to the land property value relating to land use requirement $k$ of land unit $j$, produces a membership value $y_{k}$, ranging from 0 to 1 . By using Equation $8-2$, this value is multiplied by the suitability factor $\left(l_{k}\right)$ relating to a rule's suitability level and added to the products of all the other rules to derive a land use requirement suitability value (ranging from 1 to 5). The suitability values of all the land use requirements are combined using the weighted summation procedure in Equation 8-1.

The result of the inference procedure is a value ranging between 1 and 5 that represents the average suitability of a land unit for a particular use. A land unit with a suitability value equal to 1 or less than 1 can be interpreted as being permanently unsuitable, while a value greater than 4 
can be considered highly suitable. Similarly, the other suitability levels can be derived using the ranges in Table $8-2$.

Table 8-2 Interpretations of land unit suitability values

\begin{tabular}{|c|c|c|}
\hline LEVEL CODE & LEVEL DESCRIPTION & RANGE \\
\hline S1 & Highly suitable & $>4 S_{j} \leq 5$ \\
\hline S2 & Moderately suitable & $>3 S_{j} \leq 4$ \\
\hline S3 & Marginally suitable & $>2 S_{j} \leq 3$ \\
\hline N1 & Unsuitable at present & $>1 S_{j} \leq 2$ \\
\hline N2 & Permanently unsuitable & $>0 S_{j} \leq 1$ \\
\hline
\end{tabular}

Because Equation 8-12 has an averaging effect on suitability values, an overall suitability value of 3 can be attained for a land unit with suitability values of 5 and 1 for equally weighted land use requirements $\mathrm{A}$ and $\mathrm{B}$ respectively. This means that although a land unit is considered to be permanently unsuitable in terms of property $\mathrm{B}$, its suitability level is elevated to moderately suitable by property A. In reality, however, the land unit should be considered permanently unsuitable for the particular use because it is unsuitable in terms of at least one of its properties. To ensure that the suitability value of a land unit found to be unsuitable in terms of any one of its land properties is not promoted to above 2 , the overall suitability values of all land units for which any of its properties were found to be permanently unsuitable or unsuitable at present, were reset equal to 1 and 2 respectively. This post-classification step is implemented programmatically as discussed next.

\subsubsection{Inference engine algorithm}

Based on Equations 8-9, 8-10 and 8-11, the inference engine is implemented using the algorithm set out in Figure 8-3. Upon logging in, each active user is allocated a temporary suitability item (i.e. column) in the land unit database in which calculations can be carried out. By denying other users access to this item, each user's evaluations are protected from corruption. The suitability item is automatically initialized (i.e. set all values to zero) by the system before each suitability analysis. Initialization effectively erases the results of all previous evaluations for which the suitability item was used.

The land unit database is accessed through an open database connection (ODBC), which is a standard software programming interface to connect to DBMS. To establish an ODBC, the ActiveX data objects (ADO) object database connection was used. This connection facilitates the use of structured query language (SQL) to interrogate and manipulate any database, irrespective 


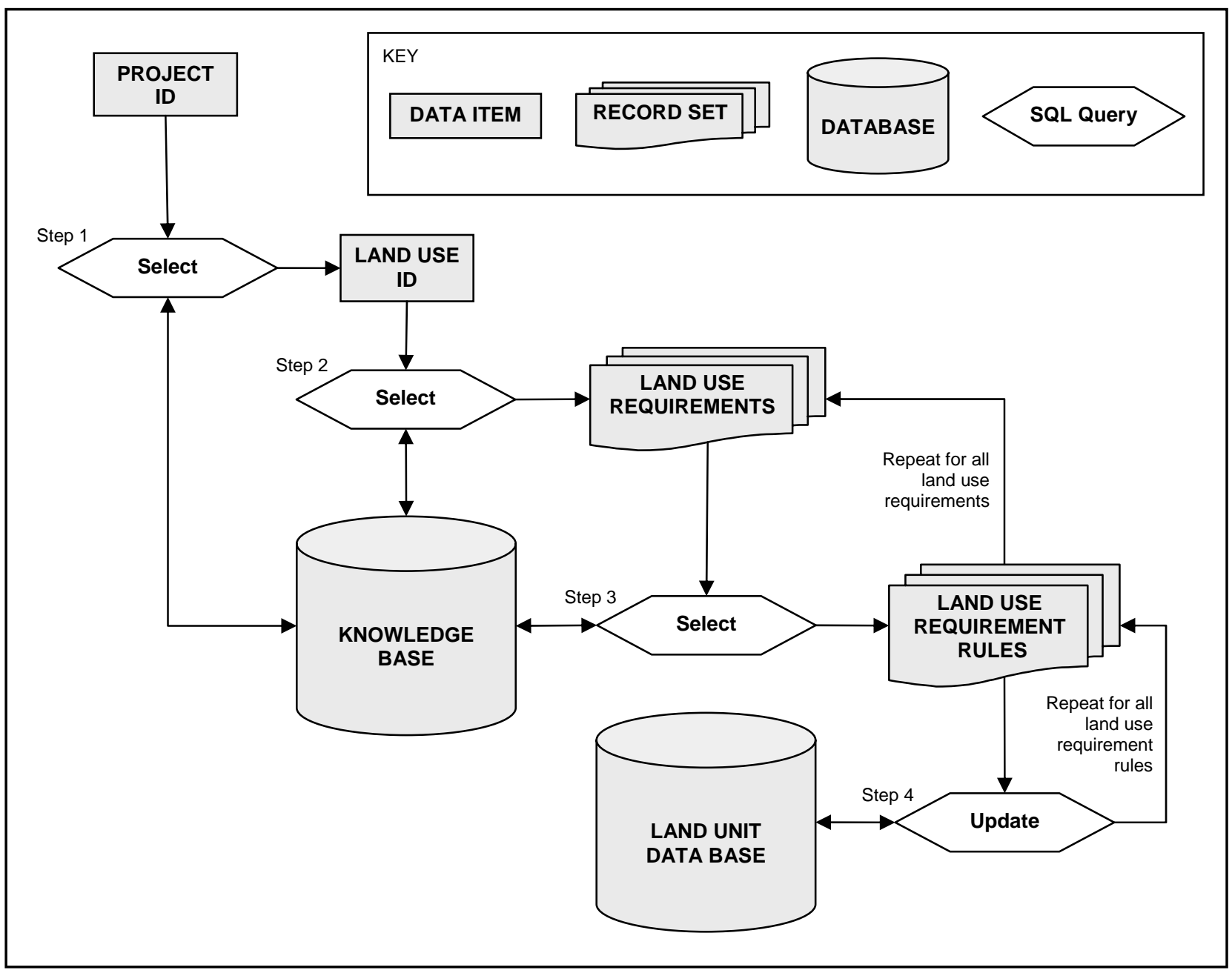

Figure 8-3 Inference engine algorithm

of the software in which it was created. By using this approach, the land unit database can easily be replaced by another database using a different DBMS without affecting the inference engine.

In step 1 of the inference engine algorithm, an ODBC is established with the knowledge base to determine which land use is to be evaluated. This information is stored in the PROJECT table of the knowledge base and is retrieved using the current project's identification number. Once the land use is known, it's identification number can be used to extract the related land use requirements from the LAND_REQUIREMENT table in step 2.

Each land use requirement is sequentially considered to calculate its individual impact on the overall suitability values of all land units. To do so, the suitability level and the type of rule as well as the lower $(\alpha)$, central $(\beta)$ and upper $(\gamma)$ values for each rule relating to the current land use requirement, are retrieved from the knowledge base in step 3. Together with Equations 8-9, 8-10 and $8-11$, this information is used to formulate the SQL statements that sequentially update the values stored in the suitability item of each land unit in step 4.

As mentioned above, the overall suitability values in the suitability item are overwritten each time an evaluation is carried out. By not storing the evaluation results, the data storage and 
management problems associated with spatial analysis are overcome. Because all the parameters of the evaluation are stored in the knowledge base, users can easily regenerate a previous evaluation. If the evaluation and mapping process is fast enough, this ability creates the impression that evaluation results are being stored.

The algorithm was implemented as a Visual Basic procedure (named CalculateSuitability), for which the code is provided in Appendix E.

\subsection{THE WEB MAP SERVICE AND WEB SERVER}

The ability to view the results of a suitability analysis as a map was identified in Chapter 2 as a principal requirement for CLUES. To enable users to interact with the land unit database in a spatial manner, a web map service (WMS) was implemented. The function of a WMS (see Section 3.4) is to convert data stored in a GIS database into a format compatible with a standard web browser. The CLUES WMS implementation involved two major tasks: software and hardware selection; and WMS configuration. These tasks are discussed in the following two sections.

\subsubsection{Choice of software and hardware}

ArcIMS version 9.2 was chosen as the CLUES WMS software, mainly because it is currently the best established WMS software available and is quite likely to enjoy continued support. It is also the WMS of choice for several governmental organizations in South Africa, including the Provincial Government of the Western Cape (Van der Merwe 2008, pers com). By using ArcIMS, CLUES will therefore be more likely to be adopted and maintained by the provincial government once the system becomes operational. Scalability is another important reason for choosing ArcIMS as this allows for future expansion of the system. It is possible to initially set up the WMS on a small server and replace it later with a more powerful server without affecting the rest of the system. The WMS can even be expanded to operate from multiple servers without making any modifications to the other components (ESRI 2003).

ESRI (2007b) recommends that separate servers be used for the spatial database (i.e. land unit database), WMS, and web server, especially when the number of concurrent users is expected to be large. However, only one server, a DELL PowerEdge 2650 was available for this project and was used for all the CLUES components. The Dual Intel Xeon $2.8 \mathrm{GHz}$ central processing unit (CPU) of the PowerEdge 2650 is significantly superior to the minimum $1.3 \mathrm{GHz} \mathrm{CPU}$ required by ArcIMS (ESRI 2007b). As for memory, the 4GB random access memory (RAM) of the PowerEdge exceeds the minimum requirements by $2 \mathrm{~GB}$. This configuration serves only for demonstration purposes and, depending on demand, the machine could eventually be replaced by 
a more powerful server, or even multiple servers. Due to the scalability of ArcIMS and the loosely coupled design of CLUES, this can be done with little effort.

\subsubsection{WMS configuration}

The configuration of the CLUES WMS entailed setting up ArcIMS and configuring the web server. An overview of ArcIMS is provided below, followed by an account of the steps taken to set up the WMS.

\subsubsection{ArcIMS overview}

The five constituent ArcIMS components are the web server, connector, application server, spatial server, and the database. Users access ArcIMS services over the Internet or intranet using alternative clients such as a web browser, ArcExplorer, ArcMap, ArcPad or mobile devices. The relationships of the different components of ArcIMS are shown in Figure 8-4.

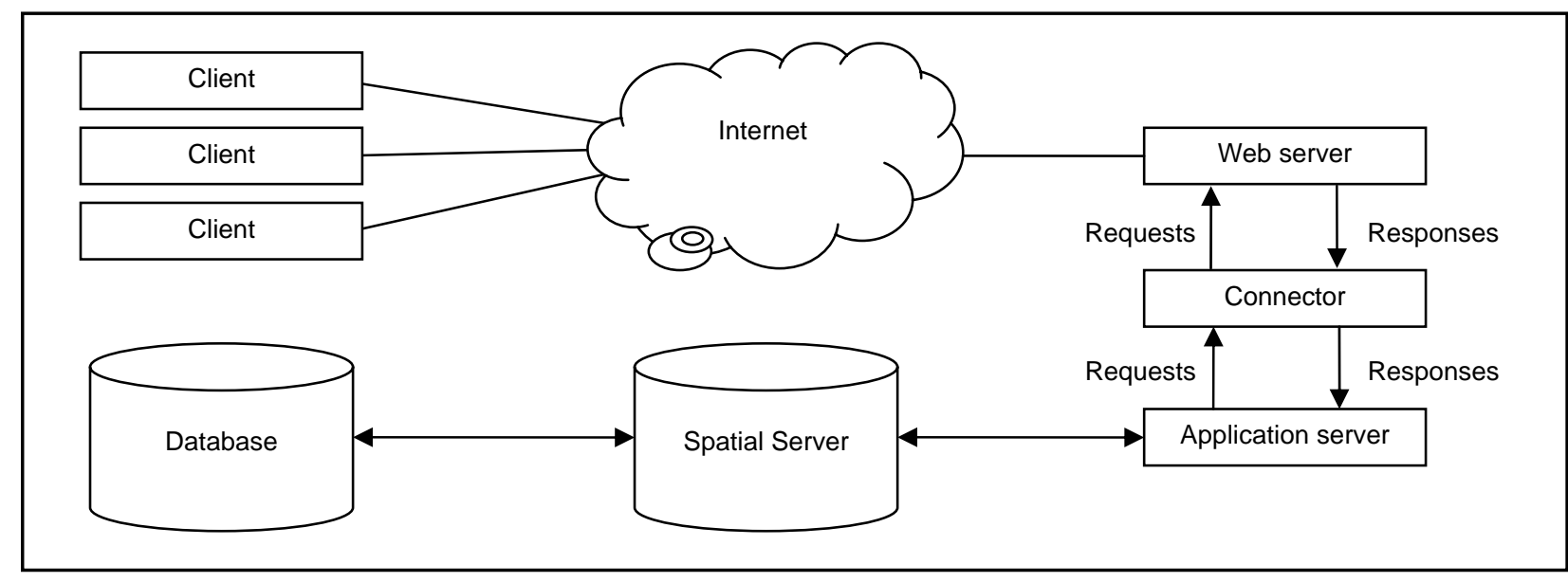

Figure 8-4 ArcIMS components

Adapted from ESRI (2007b)

The spatial data served by ArcIMS is usually stored as ESRI shape files, ARC/INFO coverages, geodatabases, images or rasters. Requests for this data are handled by the spatial server. Essentially, the function of the spatial server is to bundle the data into the appropriate format before sending the information to a client. Although other formats can also be accommodated, most web mapping applications require data to be sent as images that can be viewed using a standard web browser (ESRI 2007b).

The retrieval and conversion of data from the spatial databases is the most processing-intensive activity of the WMS. For large applications, ArcIMS can be configured to include multiple spatial servers running on separate computers to distribute the load (ESRI 2007b).

The images generated by the spatial server are sent to the application server connector via the application server. For configurations where multiple spatial servers are used, the application 
server's function is to allocate requests for data to the spatial server with the least load. This ensures that requests for data are evenly distributed among the spatial servers (ESRI 2007b).

Requests for data are sent from users' web browsers to the web server where they are interpreted by the application server connector. The default ArcIMS connector, called Servlet Engine, converts requests from the web server into ArcXML format, a special implementation of XML (see Section 3.2.2), and then sends the formatted requests to the application server for processing.

By using the Servlet Engine, ArcIMS can be rapidly configured to distribute spatial data using the preconfigured HTML and Java applications that are shipped with ArcIMS. Although these multipurpose applications are easy to configure, their functionality and flexibility are limited. Fortunately, ArcIMS includes three other connectors, namely the Web application development framework (ADF), ColdFusion and ActiveX connectors, which can be used to develop customized client applications (ESRI 2007b).

The ADF connector supports web applications and services that can be accessed from multiple GIS servers. Compatible applications and services include ESRI products such as ArcIMS, ArcGIS Server and ArcWeb Services as well as standard WMS services. Applications using the ADF connector can be developed in either the Microsoft .NET framework or on the Java platform (ESRI 2007b).

Another popular web-developing environment is ColdFusion. Applications that are developed using ColdFusion markup language (CFML) can connect to the ArcIMS application server through the ColdFusion connector which provides several tags to formulate requests for map data from the spatial server (ESRI 2007b).

Developers can also create highly customized web mapping applications using Microsoft Visual Basic and active server pages (ASP) programming languages (ESRI 2007b). Such applications can send requests to the ActiveX connector by using more than 30 predefined component object model (COM) objects. These COM objects can be used in conjunction with Microsoft's ActiveX data objects (ADO) to connect to existing databases. With $\mathrm{ADO}$, developers can write applications that access databases without any knowledge of how the database was implemented. ADO therefore allows applications to be independent of the database, meaning that a database can be replaced by another without affecting the application.

Once requests for data have been interpreted by the connector and the maps have been produced by the spatial server, the resulting images are placed on a web server for downloading. ArcIMS supports a range of web servers of which Apache and Microsoft IIS are the most popular. 
Depending on the number of requests, maps can either be placed on a dedicated web server or they could be stored with other web content on an existing server.

From the above discussion it is clear that there is a range of possible configurations of ArcIMS and that each application requires a unique approach. The configuration of ArcIMS for the development of the CLUES WMS is described in the next section.

\subsubsection{ArcIMS and web server configuration}

Due to the level of customization needed to develop CLUES, the ActiveX connector and ASP were used for developing the CLUES WMS. Seeing that the ActiveX connector is only available on Microsoft Windows platforms, the DELL PowerEdge 2650 was loaded with Microsoft Windows Server 2003. This was followed by the installation of the ArcIMS 9.2 software and licenses which were obtained from Stellenbosch University.

Because the load on the web server is unlikely to be high, a single web server was set up to support both the WMS and the GUI. Although ArcIMS is compatible with most web server software, Microsoft's internet information services (IIS) was chosen for CLUES implementation because it is the only web server software that supports the ArcIMS ActiveX connector. ArcIMS was set up to store the maps produced by the spatial server in a virtual directory called Output, which has a physical path of C: $\backslash$ ArcIMS $\backslash$ Output. Although the maps are separated from the GUI web pages, they are combined by the user's web browsers to appear in the map viewer (see Section 8.3.7)

The appearance of the maps produced by ArcIMS is determined by a map configuration file, structured in ArcXML format. The map configuration file for the CLUES application is appended (see Appendix F). The file is separated into two main sections, namely environment and map. The environment section is used to set operational parameters that are not related to the maps, like the country from where the maps are being served and the language and fonts used by the system.

The settings directly related to the maps being produced by ArcIMS are defined under the MAP section. The first setting defines the units of the coordinate system in which the data is stored. Because the data is stored in the UTM Zone 34 South coordinate system, the unit parameter was set to metres using the MAPUNITS element under the PROPERTIES subsection. Another important setting that needs to be defined in the PROPERTIES subsection is the initial extent of the map or the area that will be visible when a user opens the application for the first time. By using the ENVELOPE element, this was set to the limits of the Western Cape (see Figure 8-16). 
The next subsection in the CLUES map configuration file is called WORKSPACES and is used to define the location of the data sets and descriptions of each layer shown on a map. Although all the data is stored in a singe data folder on the server (path e:lclues\data), separate workspaces had to be specified for shape files and images as the SHAPEWORKSPACE and IMAGEWORKSPACE elements respectively. These workspaces are referenced by the definitions of each layer, which are specified using the LAYER element.

Four layers were specified. The first layer, called Suitability, is used to display the result of a suitability analysis and relates to the land unit database. To map suitability, the values in the S1 field of the land unit database are symbolized according to five suitability levels (i.e. permanently unsuitable, unsuitable at present, marginally suitable, moderately suitable and highly suitable) using the values specified in Table 8-2. To set the colours for each of these classes, the SIMPLEPOLYGONSYMBOL element is employed.

The result of an optimal land use analysis is displayed in the second or Land Use layer. The configuration of this layer is similar to that of the Suitability layer, the major difference being that the Land Use layer is set to display a nominal data set representing up to 25 land uses, as stored in the L1 field of the land unit database.

The two remaining layers are unrelated to any analysis and are included mainly for orientation purposes. Two image layers, representing a satellite image and a hillshade respectively, are specified. Both layers are set to be slightly transparent using the IMAGEPROPERTIES element. Once completed, the map configuration file was used to create an image WMS with the ArcIMS Administrator tool. Because the map configuration file includes a full-colour satellite image for orientation purposes, the JPEG file format was chosen for the output map format. This ensures that the full range of colours in the satellite image is accommodated. A compression level of $10 \%$ ensures that the images remain small enough for transfer over the Internet. Once created, the CLUES WMS was started using the Services Manager tool.

As explained above, the WMS is configured to receive requests formulated to use a range of predefined ActiveX controls. Since ActiveX controls are particularly user-unfriendly a mechanism was needed to enable users to generate requests through a user-friendly interface. The development of this facility is recounted next.

\subsection{Development of a user-friendly GUI}

The graphical user interface (GUI) was developed as a user-friendly interface to the WMS, the inference engine and the knowledge base (see Figure 4-2). The function of the GUI is to lead users through the suitability analysis procedure (see Figure 8-5). 


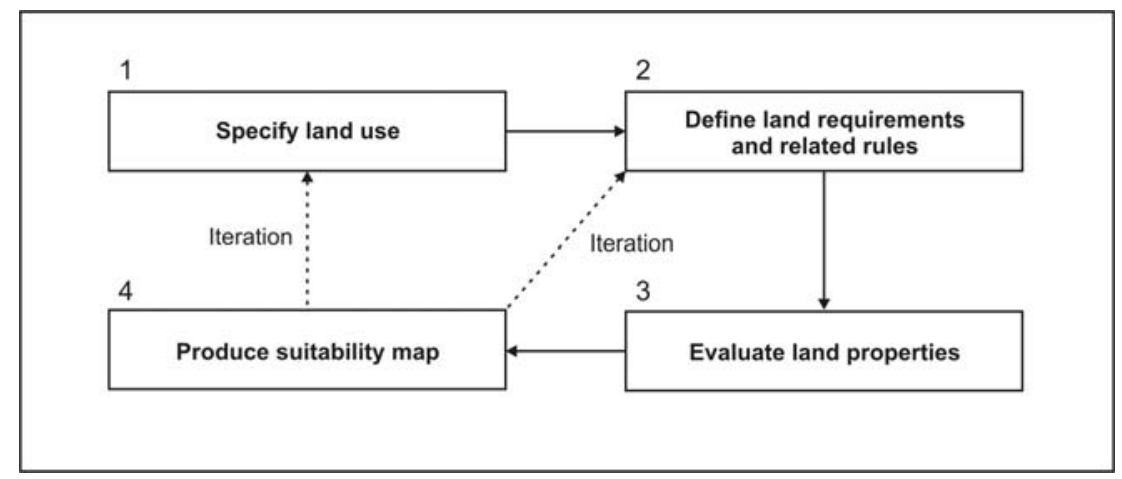

Figure 8-5 Main steps followed to produce a suitability map using CLUES

The process starts by specifying the land use that will be evaluated, followed by the defining of the land requirements and their related rules. Land properties, stored as attributes to each land unit in the land unit database, are then evaluated against the land requirements and used to calculate land unit suitability for the chosen land use. Finally, the result of the evaluation is presented to the user as a suitability map. The procedure can be repeated for multiple land uses and changes to the land requirements can be interactively evaluated and mapped in order to facilitate scenario building. For a multi-objective solution, an optimal land use map can be produced if the requirements of more than one land use are specified.

The steps shown in Figure 8-5 closely resemble steps 3, 4, 7 and 8 of the land evaluation process (compare Figure 2-1). The main difference between the two procedures is that in the CLUES process the data is already available and the user simply selects the appropriate land properties from a set of available data sets. Recall that in the land evaluation process the identification of land uses is preceded by a data collection and preparation process. By eliminating this process, users of CLUES can start with suitability assessments without the need to collect or prepare any data.

The next section describes how the GUI was structured to facilitate suitability analysis. This is followed by detailed descriptions of the six main GUI modules. The web pages that comprise each module is provided in digital format in Appendix G (a Compact Disk).

\subsubsection{GUI structure}

The GUI, shown diagrammatically in Figure 8-6, consists of 34 web pages (each represented by a rectangle) written in HTML, JavaScript and Visual Basic code. Each of these elements is required for any of the GUI web pages to function properly. As explained in Section 3.2.2, HTML code is used to format the page structure and visual appearance, while the server-side scripts or active server pages (ASP) written in Visual Basic, generate dynamic HTML code based on the status of the suitability analysis and the user (see Section 3.2.4). The resulting 
HTML is placed on the web server for downloading. JavaScript is used as client-side scripting (see Section 3.2.3) to improve interactivity.

Figure 8-7 demonstrates how the three programming elements (i.e. HTML, Visual Basic and JavaScript) interact in a page (the main page is used as example) and highlights each component as a different colour. The three components are highly integrated within each page, often interacting and exchanging data. To reduce redundancy, code used by more than one page is placed in central libraries. These libraries are named lib.asp and lib.js for the Visual Basic and JavaScript codes respectively. Refer to Appendix G (a CD) for the content of each of these files.

In Figure 8-6 it is clear that the structure of the GUI and the linkages between pages resembles the sequence of operations shown in Figure 8-5. The GUI can be divided into six main modules: login \& security; menu; user details; projects; analyse \& map; and rulebase. Each module contains one or more pages, some of which are visible and others hidden. The pages that are not visible are intermediate pages that carry out operations not associated with input or output (display).

The login \& security module acts as gatekeeper to the system and prevents unregistered users from entering. Once registered, users can log into the system using the password specified during registration. This will open the menu module, containing a single page called main. The menu acts as the hub to all the functionality of the system, directing users to the user details, projects, and rulebase modules.

In the user details module, users can change the information entered during registration. This is useful for users wanting to change their passwords or to update their email addresses.

Before an analysis can be carried out, the user must create a set of rules for each of the land uses that will be evaluated. A land use can have multiple requirements and each requirement consists of one or more rules. The rulebase module allows users to add, edit and delete individual land uses, requirements, and rules.

The projects module allows users to edit and delete existing projects or to create new projects. A project keeps record of the status and characteristics of an evaluation so that users can return to an evaluation at a later stage. Users can also work on several projects simultaneously.

Once created, a project can be opened as an interactive map through the analyse \& map module. This module encompasses the inference engine and WMS. If an evaluation has been carried out previously under the opened project, the result of that analysis is displayed. If no previous analysis is associated with a project, a new evaluation based on the land uses and rules in the rulebase can be initiated from within the mapping environment. 


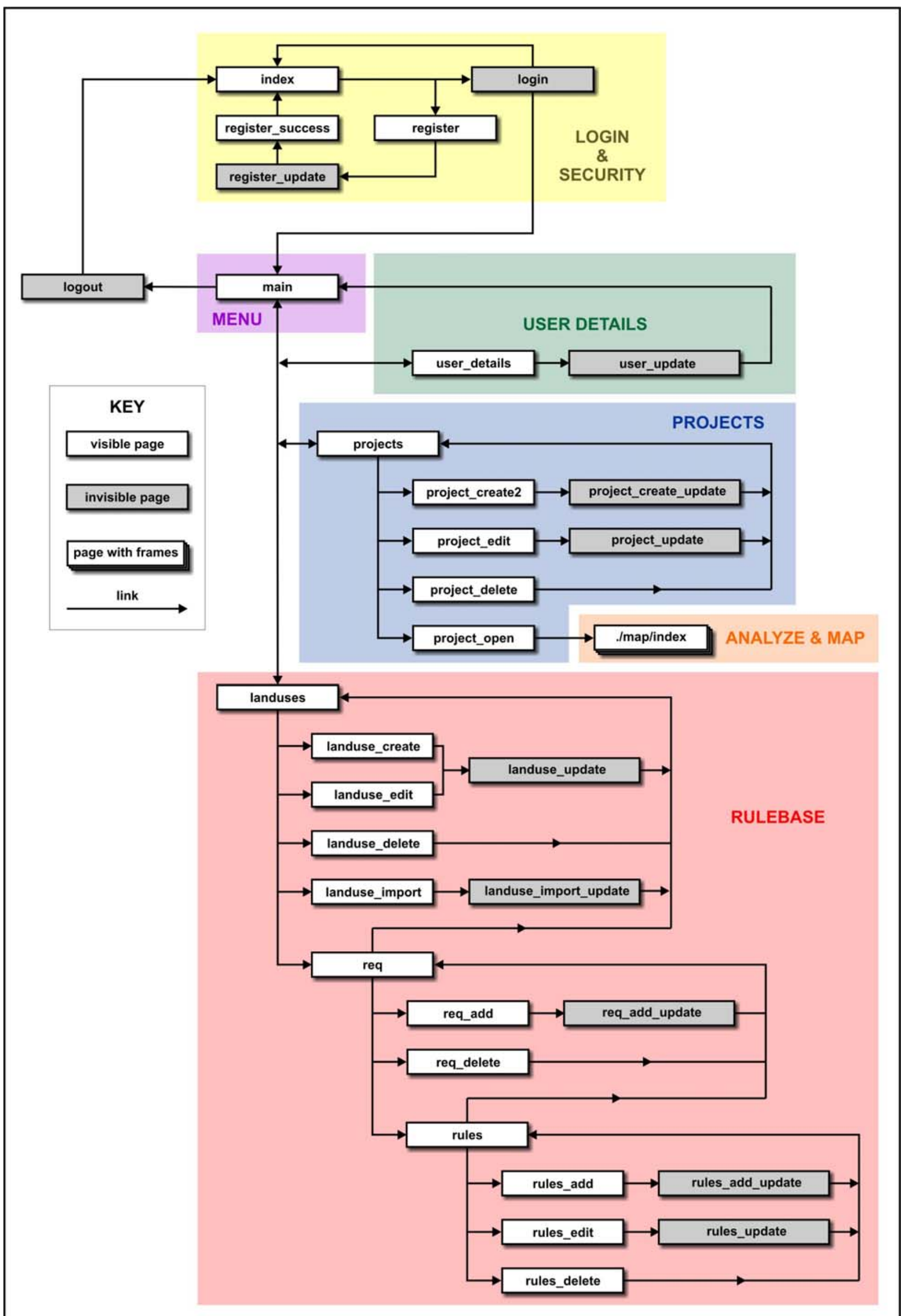

Figure 8-6 GUI pages and linkages in CLUES 


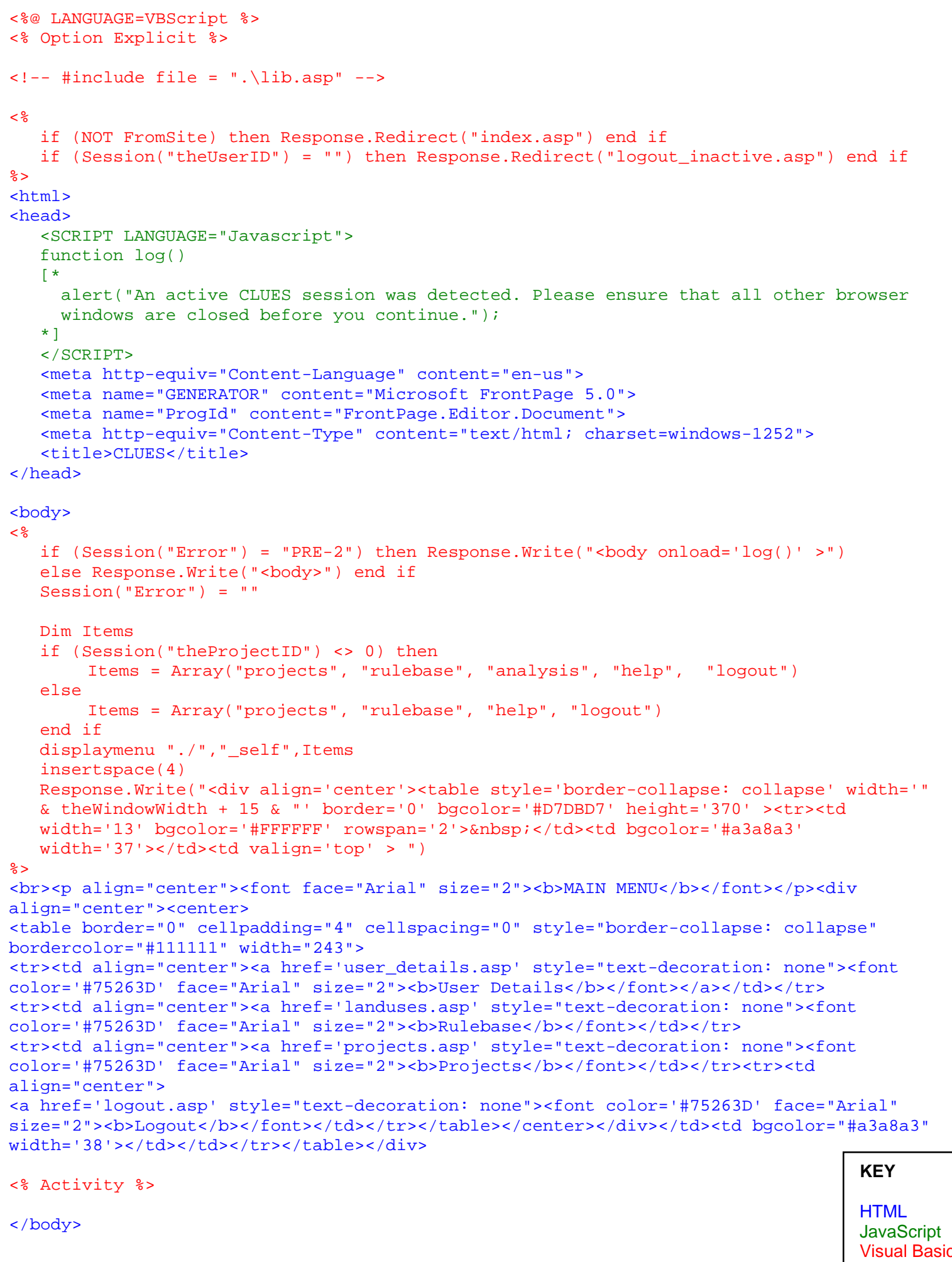

KEY

HTML

Figure 8-7 Code of the main page showing interaction between HTML, JavaScript and Visual Basic elements

In the following sections, the web pages comprising each of the six modules are described in more detail. The descriptions focus on the functions and elements of each page. No details regarding the coding are discussed and readers are referred to the full code of each page, as provided in Appendix G. 


\subsubsection{Login \& security module}

The login \& security section consists of five web pages. Upon accessing the website, users are taken by default to an index page, which acts as a doorway to the system. The index page (see Figure 8-8) contains a banner, welcoming message and login form. The banner is a standard element on all the GUI pages and is meant to provide a uniform identity, which is considered to be an important attribute of web applications (U.S. Department of Health and Human Services 2006). The welcoming message introduces the user to the system and provides instructions on how to $\log$ on. The form contains two fields where existing users can enter their username and password respectively.

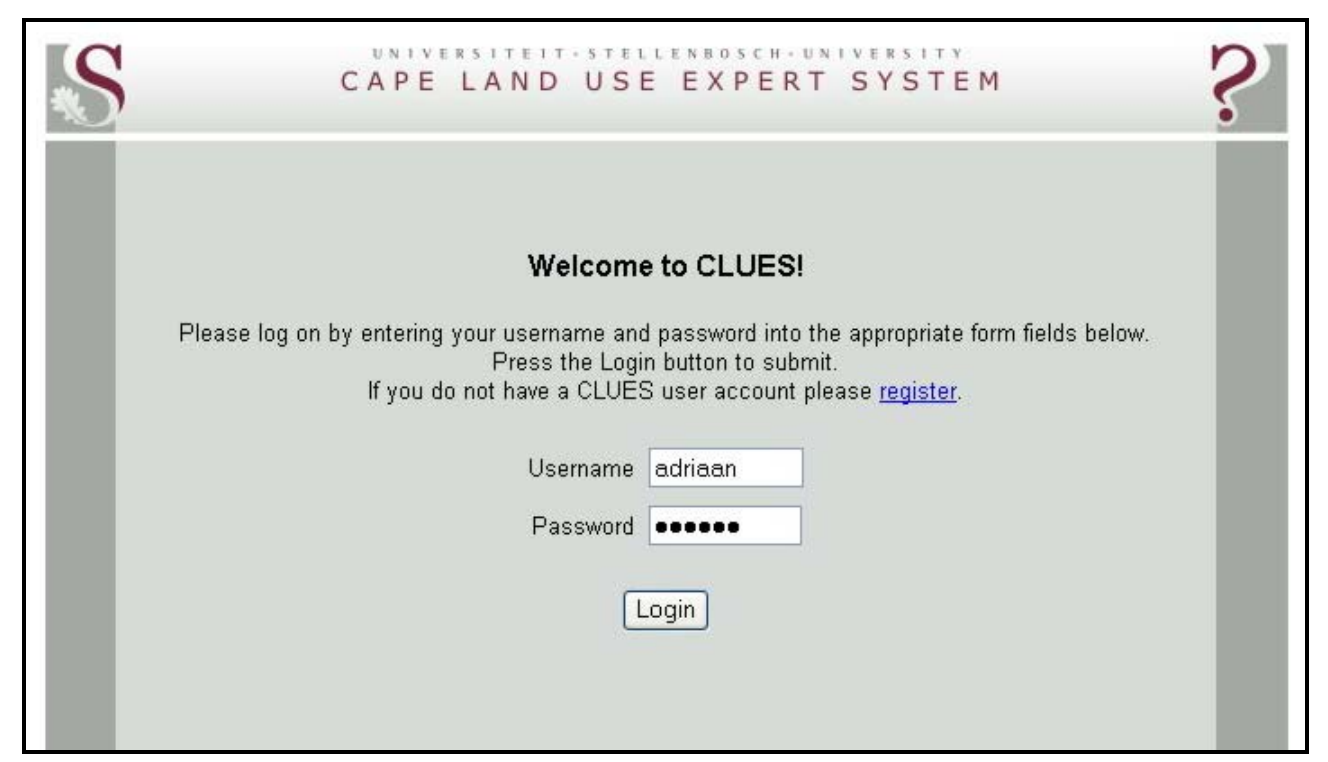

Figure 8-8 Index page of the login \& security module

Upon clicking on the login button, the information in the form is sent to the login page. The function of this page is to check whether the entered username and password combination exists in the USER table in the knowledge base. If no username and password match is found in the database, the user is redirected to the index page and no further action is taken by the login page. If a matching record is identified, users are directed to the main page.

The welcoming message on the index page also informs users that those without CLUES accounts can register by using the provided link. The link opens the register page (see Figure 8-9) on which unregistered users can create a new account. In addition to the standard header, the register page contains a form consisting of five fields: username, full name, password, confirm password and email address. All these fields in the form correspond to those of the USER table in the knowledge base. 


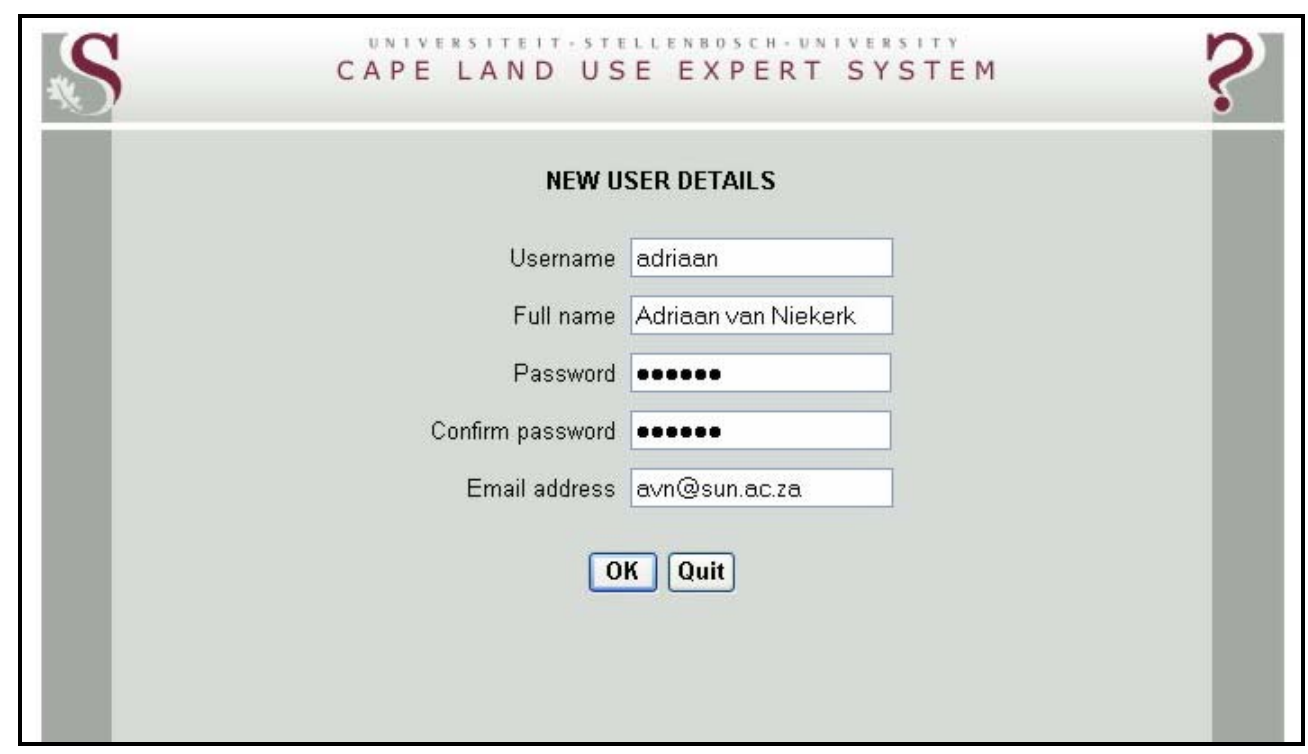

Figure 8-9 Register page of the login \& security module

Once entered into the form, the new user's information is sent to the register_update page which stores the field values into the USER table. The values are, however, first compared with those in the USER table to ensure that the username and email address do not already occur. If one or both do exist, the user is redirected to the register page and an appropriate error message is displayed. If no other records are found with the same username and email address, the user is taken to the register_success page which informs the user that the registration process was successful (Figure 8-10). The user is also provided with a link to the index page in order to log on using the newly created account details.

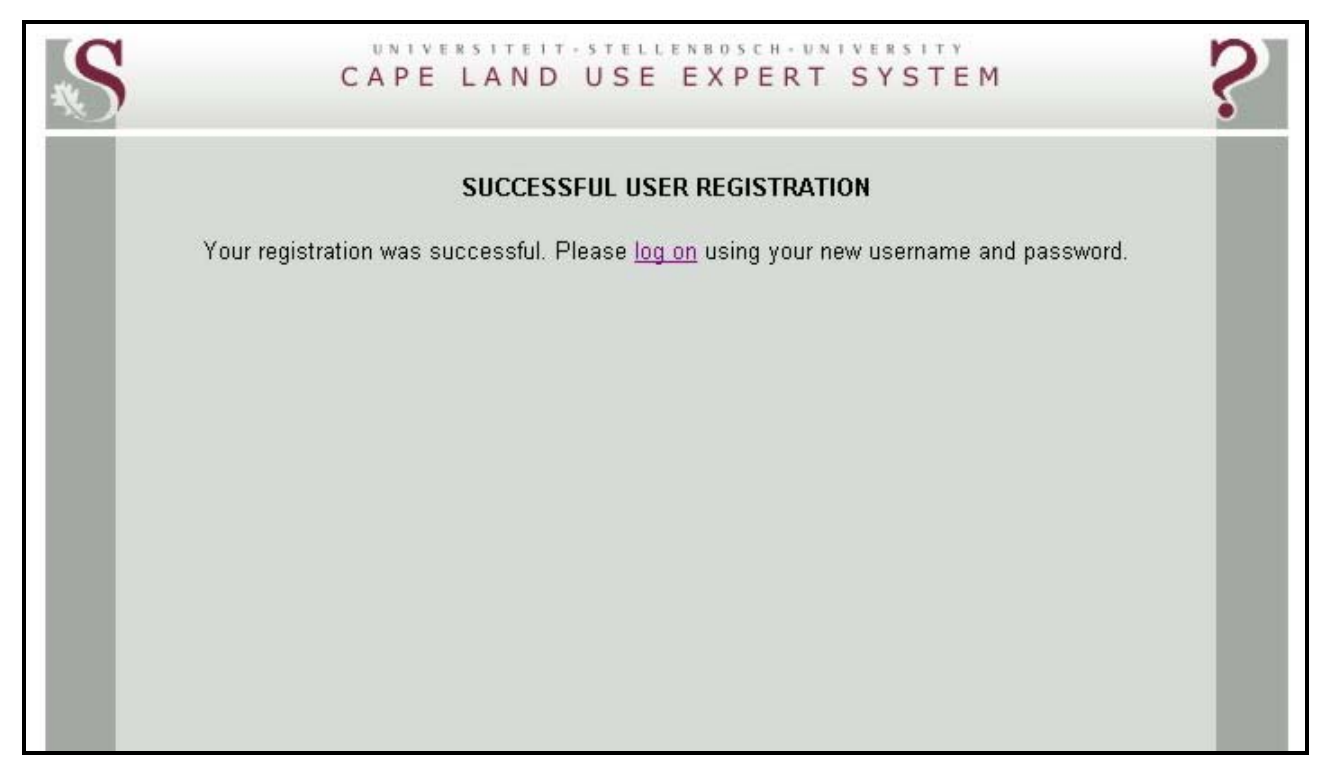

Figure 8-10 Register_success page of the login \& security module 


\subsubsection{Menu module}

Upon successful completion of the login procedure, users are taken to the main page containing the main menu (Figure 8-11). The main menu is a list of hyperlinks which direct the user to the remaining three GUI modules, namely User details, Rulebase and Projects. The user can exit the system by clicking on the Logout menu item. This will invoke the logout page, which clears all the session variables and redirects the user to the index page.

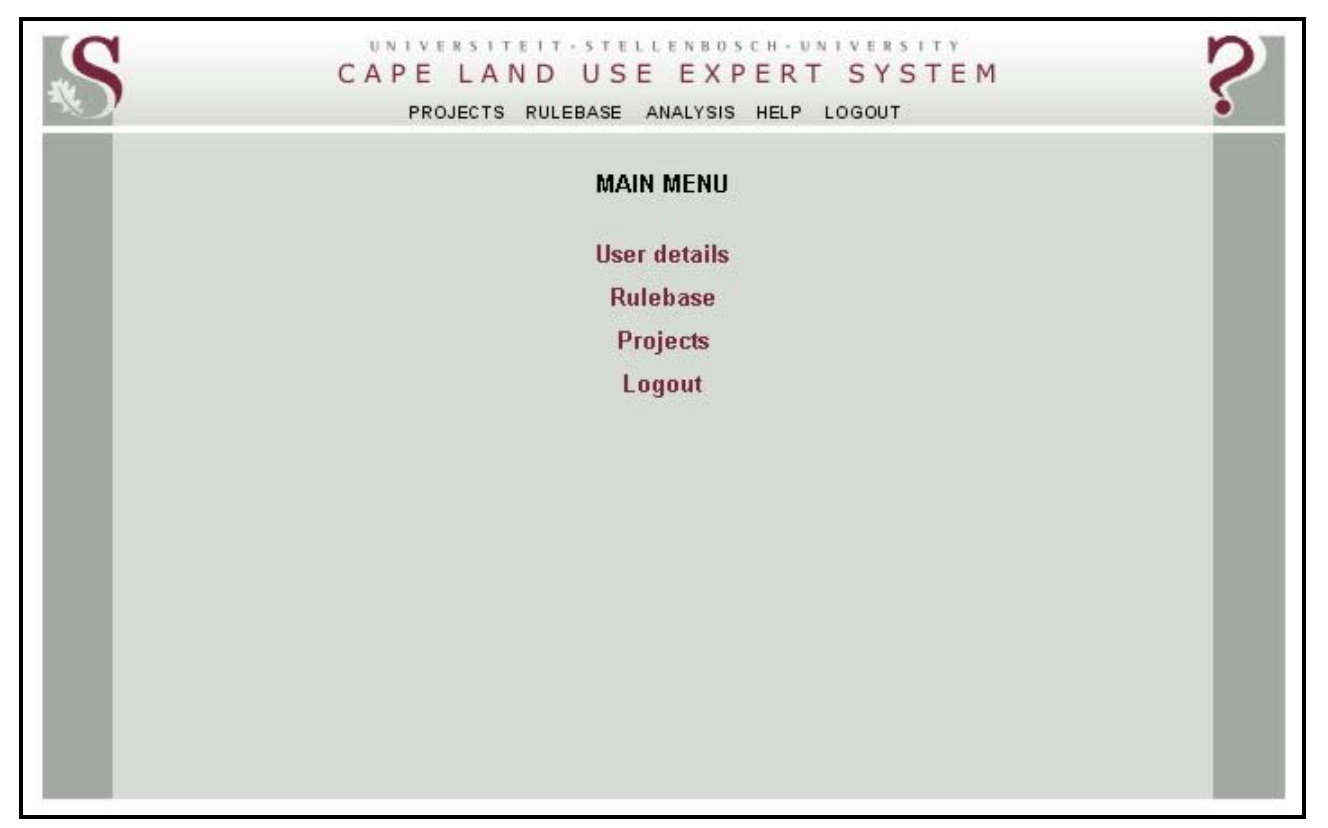

Figure 8-11 Main and banner menus as shown on the main page

Some of the items in the main menu are duplicated in a secondary menu, displayed on the banner. The banner menu is shown on most pages so that users can access these items directly without having to return to the main menu. A help item is also provided, which opens an Adobe Acrobat portable document format (PDF) file containing helpful information about the system.

\subsubsection{User details module}

The user details module contains two pages namely user_details and user_update. The user_ details page is almost identical to the register page as it also presents a form for users to update their details. The only difference between the user_details page and the register page is that the current user's details are preloaded on the former from the database so that users can view and edit their details. Upon submission of the form, the user_update page stores the updated data in the USER table in the knowledge base.

\subsubsection{Rulebase module}

The rulebase module is the most complex of all the GUI modules as it comprises 17 web pages and more than 1500 lines of code. To structure the description of these pages, the module is 
subdivided into three sections, namely those dealing with specification of land uses, land use requirements, and land requirement rules. These subdivisions relate to the landuses, req and rules pages respectively (see Figure 8-6 for system location) and are described separately below.

\subsubsection{Land uses specification}

The first page shown upon entering the rulebase is the landuses page (Figure 8-12). This page relates to the LAND_USE table in the knowledge base and lists all the land uses owned by the current user.

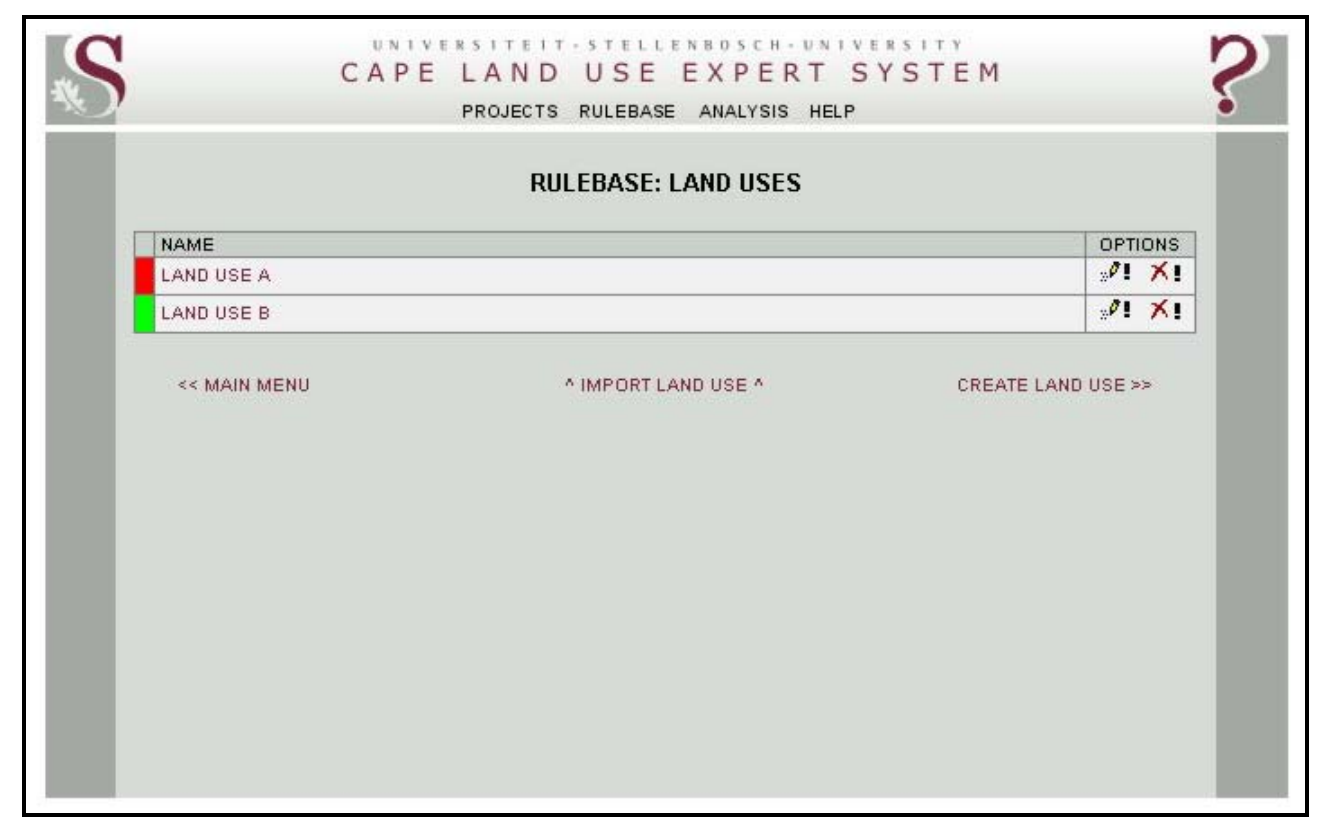

Figure 8-12 A list of land uses owned by the current user shown on the landuses page

Two items are available under the OPTIONS column for each land use. By choosing the edit (4) option, the landuse_edit page is opened. In addition to the information shown in the landuse page, this page contains a form through which the user can edit the current land use name. Once submitted, the information in the form is sent to the landuse_update page where it is stored in the LAND_USE table of the knowledge base. The delete $\left({ }^{\prime}\right)$ item in the OPTIONS column opens the landuse_delete page, which simply removes a land use from the LAND_USE table and the related rows of the LAND_REQUIREMENT and LAND_REQUIREMENT_RULE tables.

Users can create new land uses by using the CREATE LAND USE link. This opens the landuse_create page, which allows the user to enter the name of a new land use into a form. When submitted, the landuse_update page is opened inserting a new record into the LAND_USE table and setting the NAME field to the value entered.

In addition to a link back to the main menu, a function by which users can import land uses is also provided. The IMPORT LAND USE link opens the landuse_import page, which displays a 
list of land uses that are stored in the LAND_USE table and owned by the Administrator (USER_ID = 1). Once selected, the landuse_import_update page is activated to create a duplicate row in the LAND_USE table of the knowledge base and to change the ownership of the duplicate land use to that of the current user. The duplication process is repeated for all the land requirements and rules related to the specific land use.

\subsubsection{Land use requirements specification}

Each of the land use names in the land use list hyperlink to the req page which contains the land use requirements for a particular land use (see Figure 8-13). Users can add requirements by clicking on the ADD REQUIREMENT link on the page. This opens the req_add page which lets the user select a requirement from a list of available land use properties in the LAND_PROPERTY table of the knowledge base. The selected property is added to the current land use requirements list by the req_add_update page.

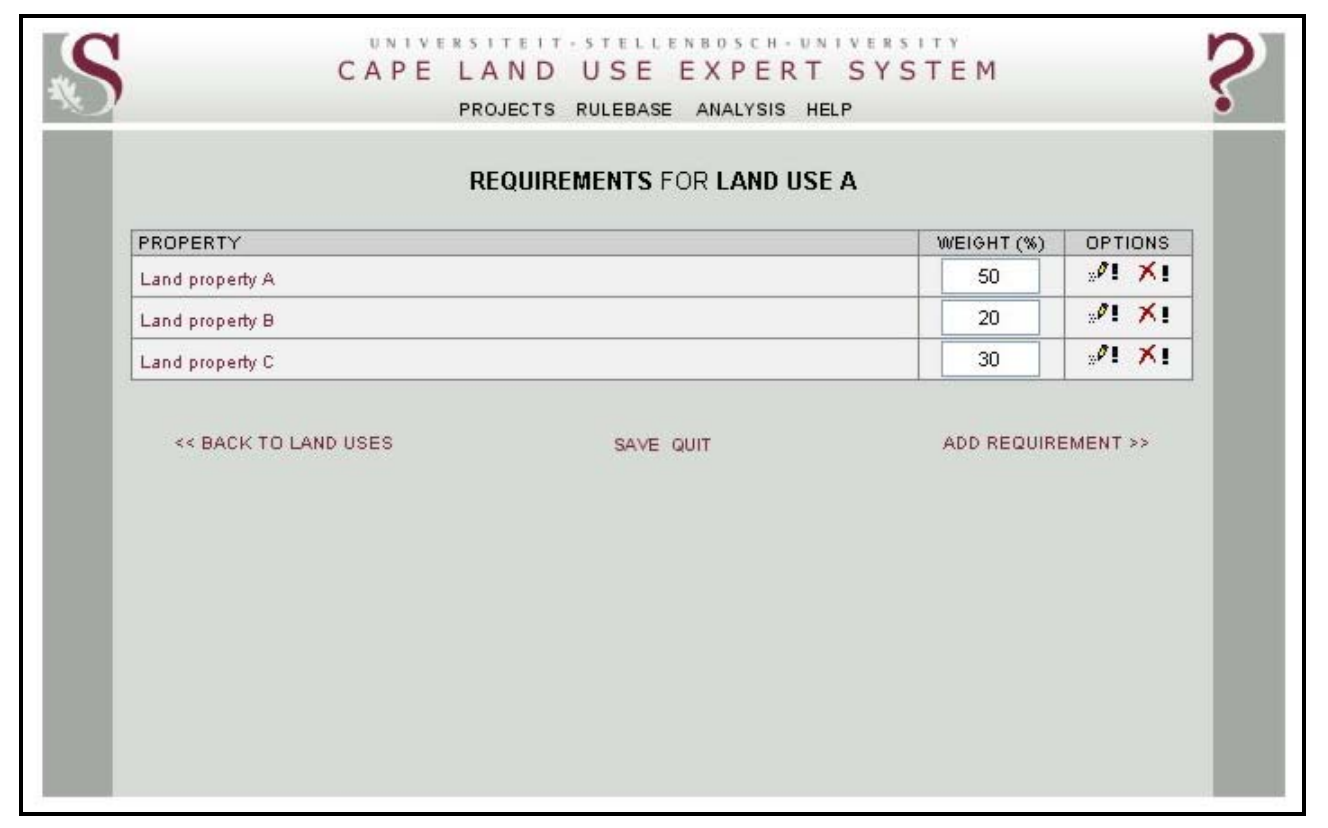

Figure 8-13 Requirements for land use A as listed on the req page

The relative importance of each requirement is shown in the WEIGHT (\%) column of the land requirements list. These weights can be edited directly and saved by clicking on the SAVE function. The list also includes an OPTIONS column enabling users to edit or delete any of the listed land use requirements. These two items open the rules and req_delete pages respectively. The req_delete page is similar to the landuse_delete page in that it simply removes the relevant rows from the LAND_REQUIREMENT and LAND_REQUIREMENT_RULE tables. To open the relevant rules associated with the listed requirements, the user can either click on the edit option under the OPTIONS column or simply click on the property name. At any time users can 
select the QUIT link to exit the page. Exiting will discard any changes made to the land use requirements.

\subsubsection{Land use requirement rules specification}

The rules for each land use requirement are shown on the rules page. Figure 8-14 lists six rules defining the hypothetical Land Use A. The requirement includes one symmetrical, fuzzy rule for the highly suitable, moderately suitable, marginally suitable and unsuitable at present suitability categories, while the permanently unsuitable suitability level is defined by two rules. The first of these is an asymmetrical fuzzy rule ranging from $60 \%$ to $80 \%$, while the second constitutes a Boolean rule specifying that all values greater than 80 are considered permanently unsuitable. Although the lower threshold of the sixth rule is equal to the upper threshold of the fifth rule, the value stored in the latter is interpreted by the inference engine as 'less than' 80 . The two values are therefore mutually exclusive.

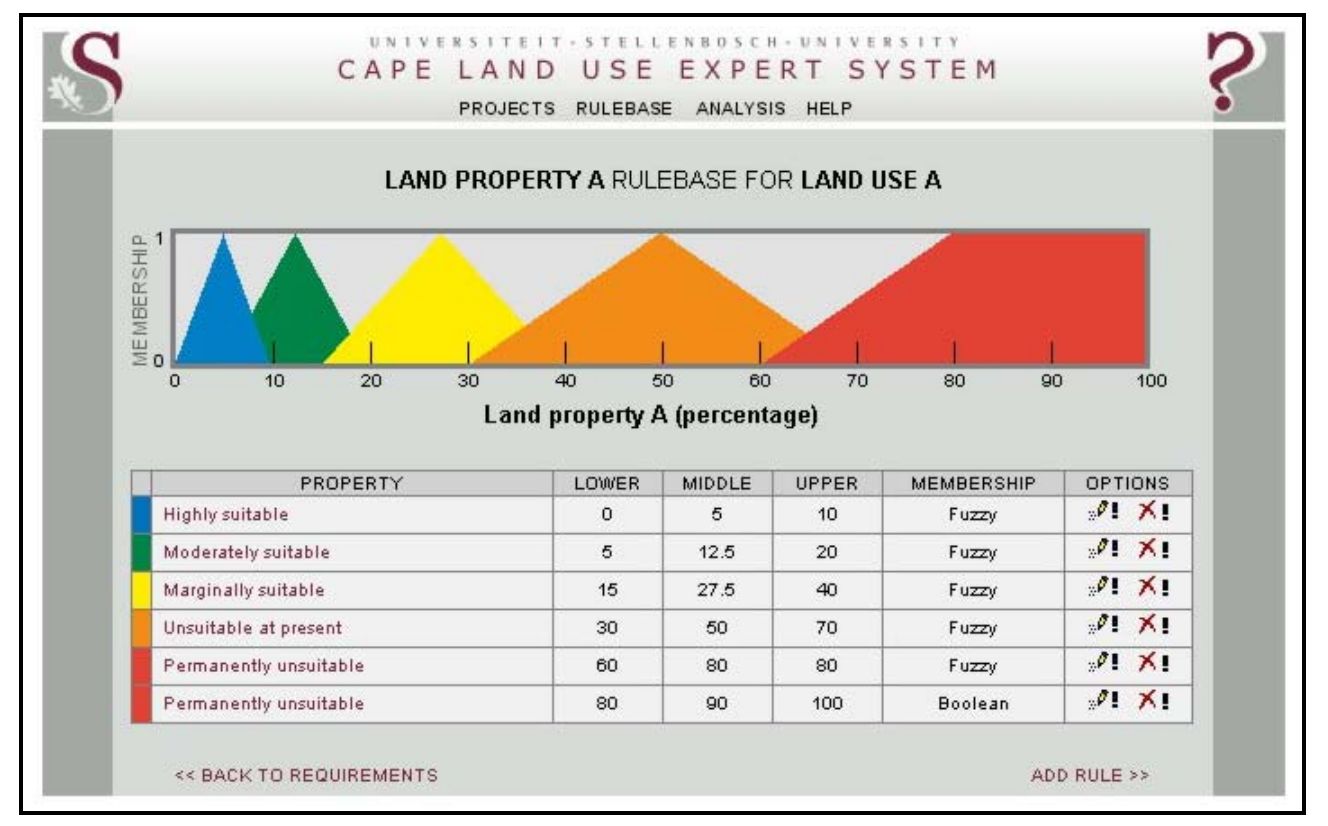

Figure 8-14 Requirement rules for a hypothetical land use as displayed on the rules page

The six rules listed in the table are also illustrated diagrammatically so that users can visualize the specified thresholds and fuzzy functions. The diagram was challenging to implement and considerable time was invested in its development. Although the diagram is functional and is rendered quickly, it has a number of limitations. Probably the most noticeable is the opaqueness of the shapes representing the different suitability levels, causing some rules to be partly obscured by others. Each fuzzy function is composed of one or more images and because current web browsers are unable to render transparent images, this shortcoming could not be overcome. To compensate for this limitation, the order in which the rules are drawn in the diagram can be manipulated by moving the mouse over the property name in the table. Another problem with 
current technology is that only rectangular and triangular shapes could be used to emulate the forms of the fuzzy functions. Functions that curve, such as s-functions, can not be depicted.

Rules can be deleted and edited in the same way described for requirements and land uses. New rules can be added by using the ADD RULE function on the page or the user can return to the req page by clicking on the BACK TO REQUIREMENTS function.

\subsubsection{Projects module}

Users can store the results of a particular suitability analysis done in a project. A user's projects can be viewed on the projects page (see Figure 8-15). The page lists all the projects along with the date and precise time a project was last modified. Users can also edit and delete projects using the tools in the OPTIONS column and new projects can be created by using the NEW PROJECT function.

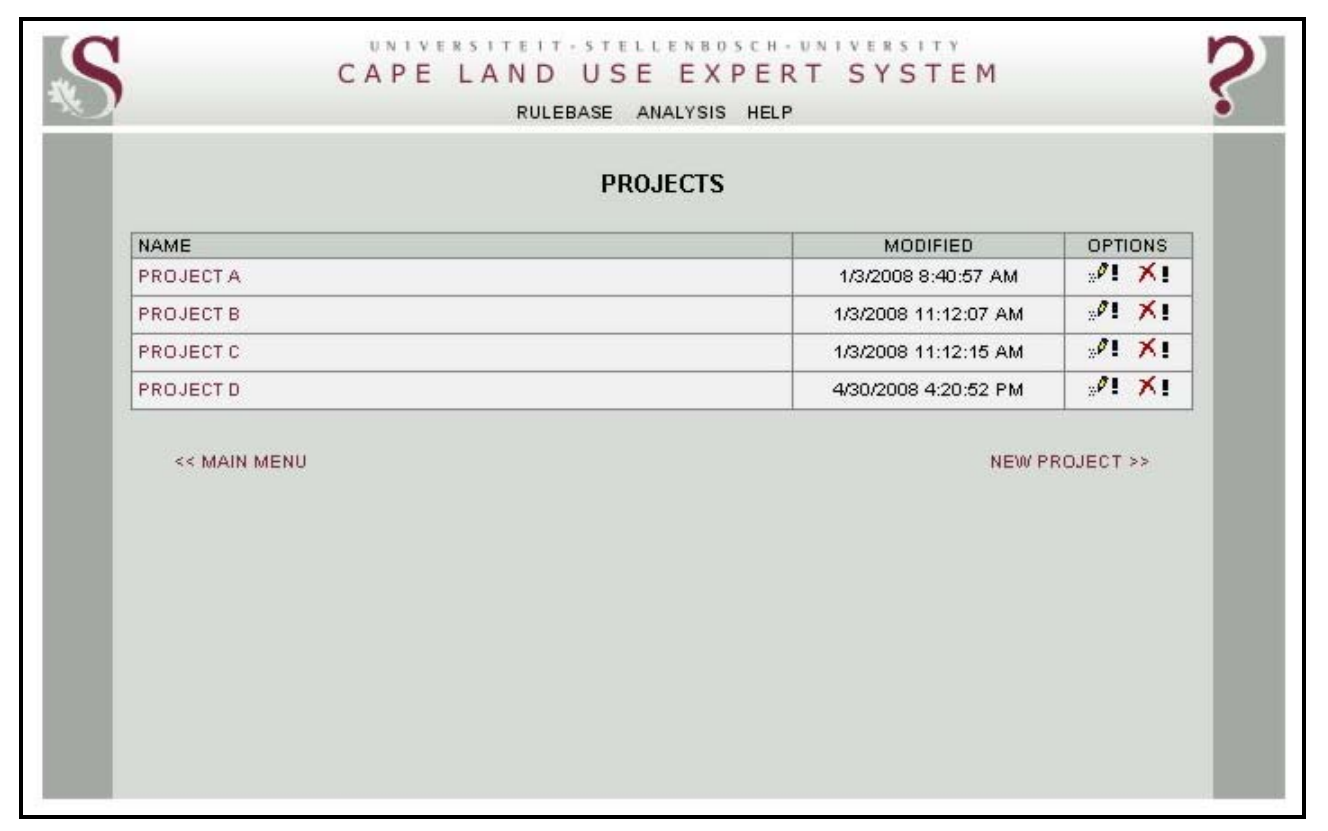

Figure 8-15 Information about a user's projects shown on the projects page

As mentioned in Section 8.3.3, each GUI page includes a banner with a menu that acts as a short cut to the modules. The banner menu is customized for each page to include relevant items only. While some items, such as RULEBASE and HELP, are standard on all pages allowing users to open the rulebase and online manual at any time, other items are only present under certain conditions. For instance, the ANALYSIS item which opens the analyse \& map module is only available if a project has been opened during the current session. The analyse \& map module can also be opened by clicking on a project name in the NAME list. 


\subsubsection{Analyse \& map module}

The analyse \& map module consists of one page which acts as an interactive map viewer. In reality, the viewer consists of many pages located in frames within the viewer page (called map/index). A frame is an HTML element that facilitates the inclusion of web pages in an existing web page. Frames are often used for web mapping applications to emulate GIS viewers. Such viewers usually contain a map area, where the spatial information is rendered, and a menu allowing users to select tools to interact with the map (e.g. zoom and pan). Many applications also include a table of contents (TOC) listing available data layers. Users can use the TOC to select the layers to be displayed. When included in web mapping applications, such elements are usually implemented as separate pages loaded into predefined frames. The advantage of this approach is that only certain frames need to be refreshed once an action is taken.

Five web pages and frames were used to develop an integrated analysis and mapping environment for CLUES. The first frame, called top, holds the banner and spans the entire width of the viewer (see Figure 8-16). As with the other GUI pages, the banner includes menu items that can be used to quickly access other functions. In the mapping environment, this feature is especially useful when a land use requirement rule needs to be edited and re-evaluated.

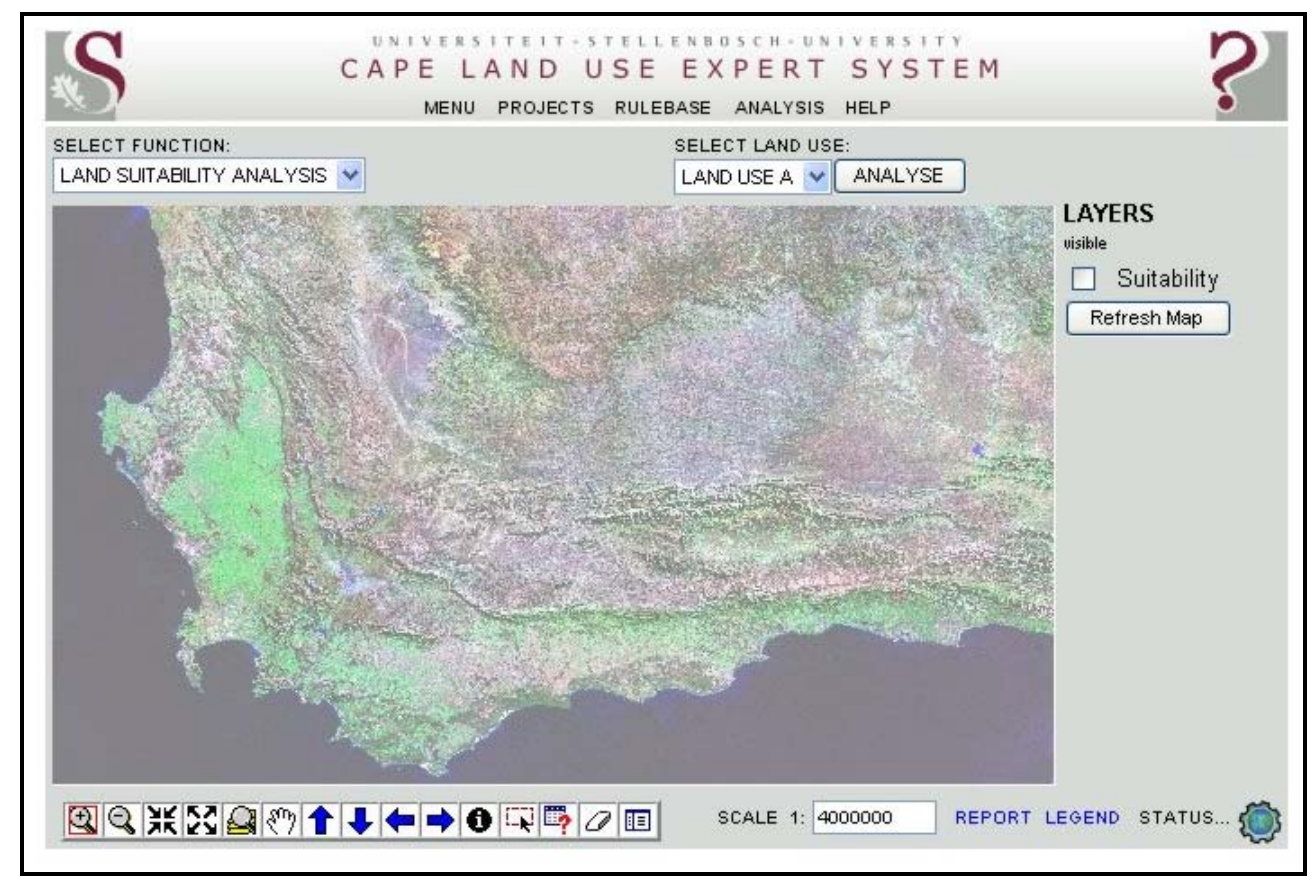

Figure 8-16 CLUES map viewer

The menu frame, positioned directly below the banner, was implemented to accommodate the analysis menu. The analysis menu contains two drop-down lists and a button. From these two lists the user can select the type of analysis and land use to be evaluated respectively. Although only one analysis function is currently available, it is expected that more functions will be added 
as the system is expanded. The land use drop-down list contains all the land uses in the user's rulebase and the GO button initiates the analysis.

The frame containing the map information is called map and is, at $565 \times 330$ pixels, the largest of all the frames. In addition to the map data, the map frame includes a north arrow and a line scale. The map frame can be manipulated by the range of tools in the tool frame, which is positioned directly below the map frame. Table 8-3 describes the functions of each of the available tools. The tool frame includes a map scale, which can be edited by the user to change the scale of the displayed map. If any of the land units on the map are currently selected, a report can be opened and printed using the REPORT function, while the LEGEND link opens a key for the current map. To ensure that users are informed about the status of any action, a status indicator is provided to the right of the tool frame.

Table 8-3 Tools available for manipulating the map frame

\begin{tabular}{|c|c|}
\hline Tool & Description \\
\hline 9 & Zoom out using mouse \\
\hline Q & Zoom in using mouse \\
\hline 彭 & Decrease scale by a factor of two \\
\hline ky & Increase scale by a factor of two \\
\hline 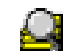 & Zoom to the Western Cape \\
\hline$g$ & Pan using mouse \\
\hline & Pan up \\
\hline & Pan down \\
\hline & Pan left \\
\hline & Pan right \\
\hline & Show information about a particular land unit using the mouse \\
\hline 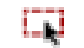 & Select land units by drawing a rectangular area and show information about all the selected land units \\
\hline 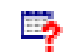 & Build a query \\
\hline$\theta$ & Deselect all land units \\
\hline 圆 & Show a legend \\
\hline
\end{tabular}


The layers frame is directly above the status indicator and lists the layers currently being displayed. In addition, this frame allows users to manipulate the visibility of layers by switching layers on or off. A refresh map button is included to effect any changes made to the layers list.

Although the frames described here are all individual web pages, they are integrated through programming to work as a unit. Many actions carried out in one frame will cause a reaction in another frame. For more details about the implementation of the analyse \& map module the reader is referred to the source code in Appendix G, which includes detailed comments and explanations.

This chapter described the development of the CLUES website, consisting of a GUI, inference engine and WMS. The GUI enables users to store a set of land requirements and rules in the CLUES knowledge base. During a suitability analysis, the knowledge base is interrogated by the inference engine to calculate land use suitability values for each land unit in the land unit database. The suitability values are then used to dynamically produce suitability maps which are converted by the WMS to a format that is viewable using the GUI. The GUI, inference engine and WMS form a coherent unit and enable users to carry out suitability analyses in a userfriendly manner demonstrated in the next chapter. 


\section{CHAPTER 9: DEMONSTRATIONS OF CLUES}

CLUES was developed to demonstrate how web technology can be used to deliver spatial analysis and modelling capabilities to Internet users. The resulting software product is a working web-based land evaluation system for carrying out land suitability analysis in the Western Cape. Land evaluation was the chosen application field because it strongly relies on geographical data and spatial analysis. In addition, there exist a need for a system that will support decisions regarding the optimal use of the Western Cape's land resources. Sound land use planning is required to ensure that the Western Cape's land and environmental resources are used sustainably.

To support decisions about the optimal use of land, this chapter demonstrates how CLUES can be used to generate suitability maps for perennial crops. Perennial crops constitute a major land use (see Section 2.1.3) encompassing many types of fruit and vines, but exclude annual commodities such as vegetables and wheat. Annual crops are excluded in this study because their cultivation practices and requirements differ markedly from those of perennial crops (Reiger 2006). Although the requirements specified in this chapter pertain to the main perennial crops produced in the Western Cape, namely deciduous fruits, vines, citrus and olives, it can be easily modified to include other crops.

To perform suitability analyses using CLUES, users are required to define the requirements of each land use to be assessed. Land use requirements are defined by setting land requirement rules, consisting of suitability levels, functions and thresholds for each land property considered. Because land use requirements seldom have equal importance, the relative importance of individual land use requirements can be specified by allocating a weight to each requirement. To conclude the suitability analysis procedure, a suitability map is produced showing the different levels of suitability for a particular area.

The following sections demonstrate how CLUES can be used to produce suitability maps for perennial crops. The defined rules are based on the specific crop requirements recorded in the literature. Only those fundamental land properties available in the land unit database were considered for the requirement definitions. The steps taken in the suitability analysis are illustrated using computer screen captures of the system's interface. Some maps are however supplemented with locational information (e.g. town names) for orientation purposes and do not appear on screen. Suitability maps are also provided with a standard legend which CLUES displays as a separate window. 


\subsection{SETTING RULES FOR PERENNIAL CROPS}

In order to set rules for perennial crop suitability, a new land use needs to be created in the knowledge base. To do so, the rulebase was opened from the main menu (Figure 8-11) to access the land uses page (Figure 8-12). A new land use called Perennial Crops was created using the CREATE LAND USE link. This link opens the landuse_create page allowing one to enter the name of a new land use (Figure 9-1).

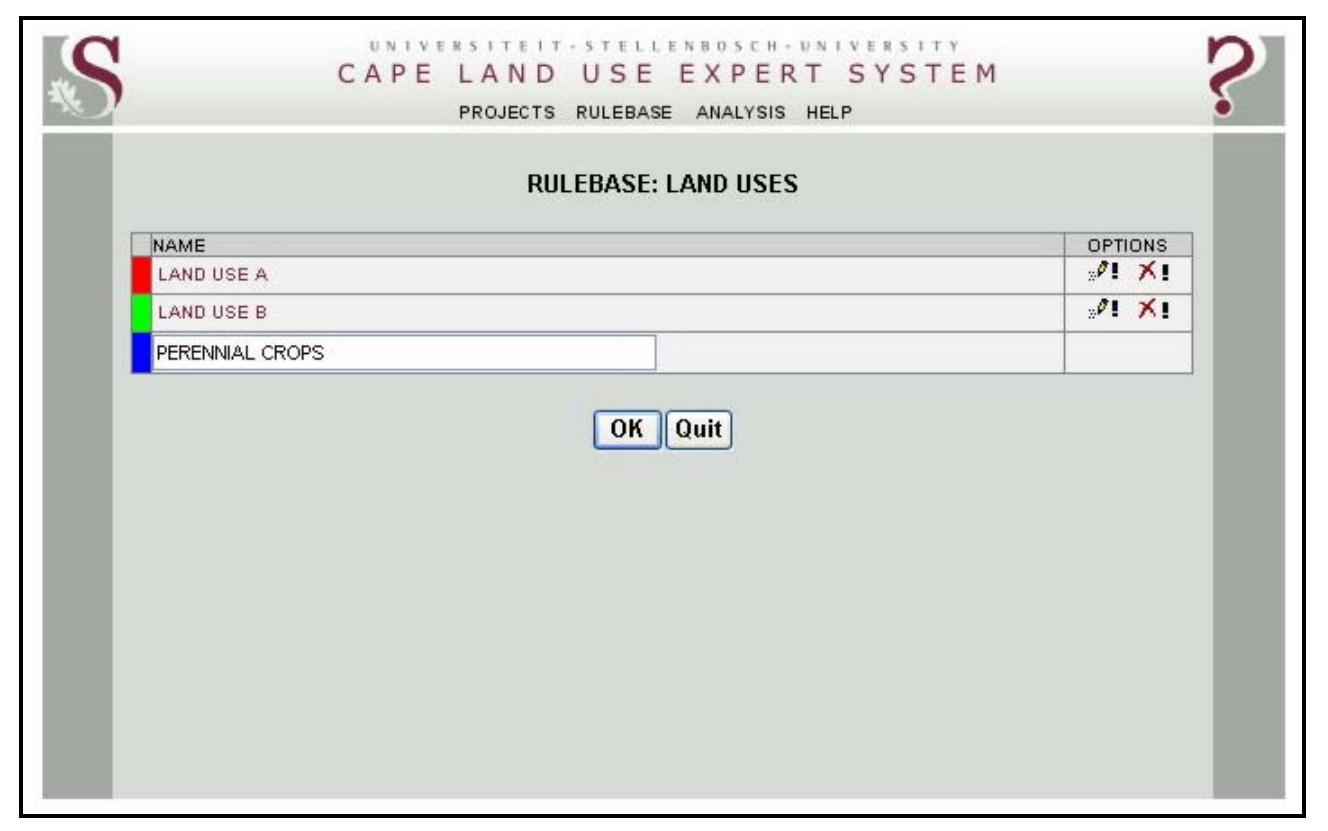

Figure 9-1 The landuse_create page used to create a new land use called 'perennial crops'

Once the new land use has been created, the knowledge base is prepared to accommodate land use requirements related to perennial crops. The steps taken to set up the necessary rules are discussed in the following sections.

\subsubsection{Terrain requirement rules}

Terrain has both direct and indirect influences on most agricultural land uses because it relates to climate and soil (see Section 4.1.3.4). The terrain-related factors considered for perennial crops are slope gradient, aspect, curvature and elevation.

\subsubsection{Slope gradient rules}

According to the regulations of the Conservation of Agricultural Resources Act no. 43 of 1983 (South Africa 1984), no land with slopes steeper than 20\% may be ploughed without permission from the appropriate authorities. Land units with slopes of more than $20 \%$ should therefore not be considered for perennial crops and hence are excluded from further analysis. In general, 
slopes with lower gradients are more suitable for perennial crop production, simply because it is easier to work the land on gentle slopes and because soils on steeper terrain are mostly shallower and dryer than those in areas that are gently inclined.

To install a rule that will exclude land units with slopes of more than $20 \%$ in CLUES, the Perennial Crops requirements page was opened by clicking on the appropriate link on the landuses page shown in Figure 9-1. The next step was to add a new item to the requirement list by selecting the ADD REQUIREMENT link. This opens the req_add page which contains a drop-down list of all the land properties in the knowledge base (see Figure 9-2). By referring to this list a user can select properties from a range of land properties to be included in a suitability analysis. To define a rule for slopes, the Slope gradient (\%) item was selected.

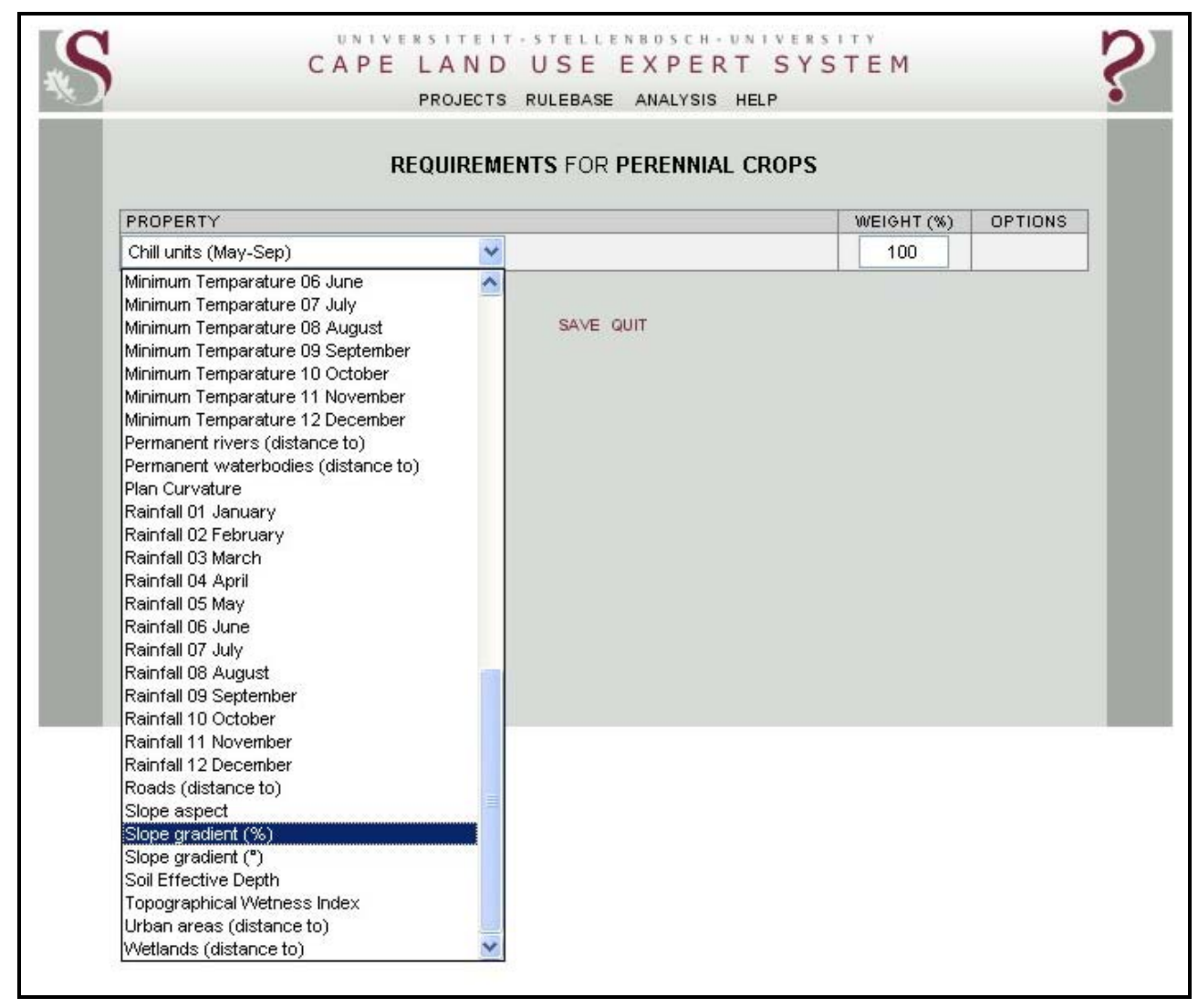

Figure 9-2 Slope gradient (\%) added as a requirement for perennial crops

Once created, the new rule was added to the slope land use requirement by opening the rules_add page using the ADD RULE link on the rules page. As explained in Section 8.1.1, when a land unit is classified as being permanently unsuitable for a particular land property, it cannot be promoted to a higher suitability level, no matter how well it performs any of its other land properties. A rule with a suitability level of permanently unsuitable is therefore regarded as a constraint, while suitability levels other than permanently unsuitable are considered as factors. Factors are rules that enhance or detract from a land use's overall suitability, while constraints are meant to limit or exclude areas for consideration (Malczewski 1999). 
To specify a constraint in terms of slope gradient it was appropriate to specify a Boolean rule with a suitability level of permanently unsuitable. A Boolean rule was chosen because it makes a hard distinction between land units on slopes of more than $20 \%$ and those that are not. To create the rule, the lower threshold for permanently unsuitable was set to $20 \%$, while the upper threshold was set to the maximum slope value that occurs in the land unit database (i.e. 207\%). The diagram for the rule is shown in Figure 9-3. Although the middle value (i.e. 113.5\%) is not used in Boolean rules, it is automatically calculated by the system in case the membership function is changed.

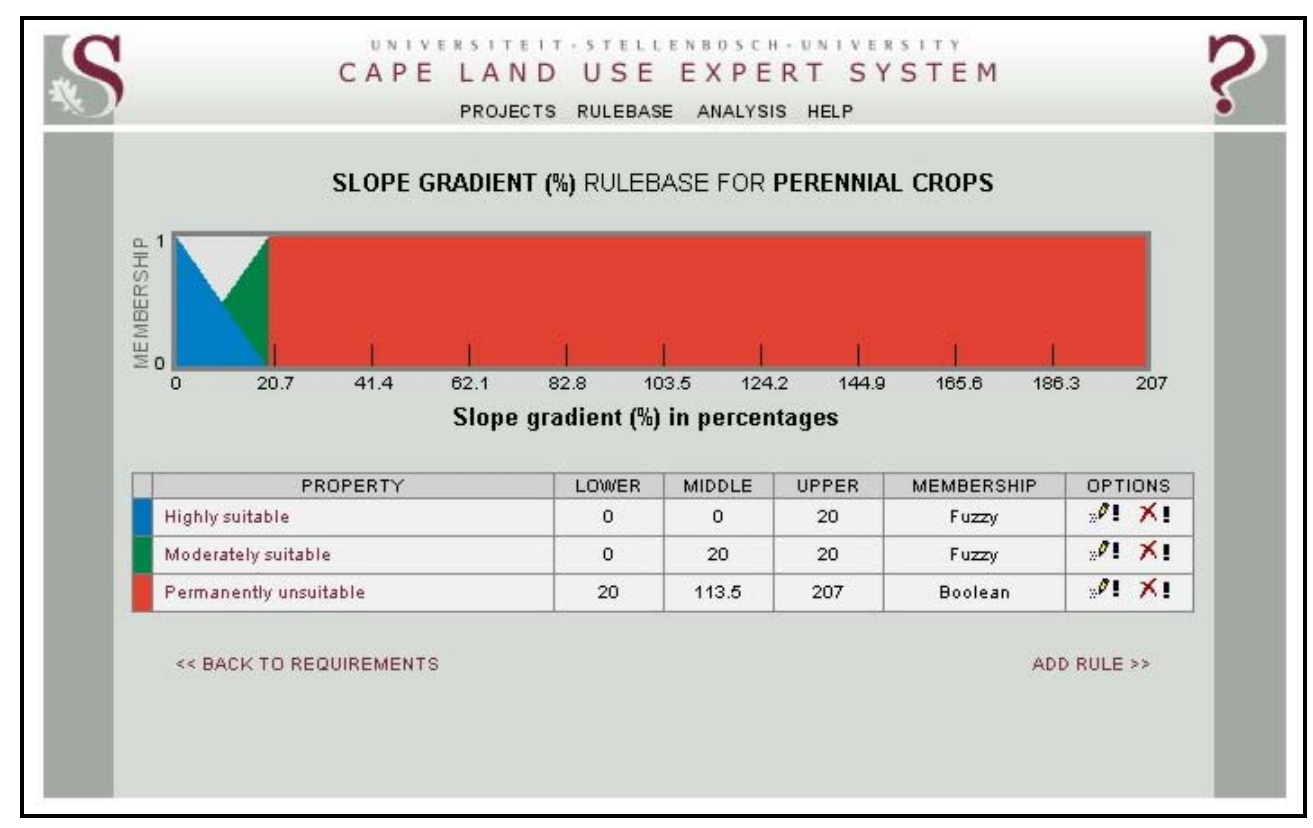

Figure 9-3 Rules defining suitability levels for slope gradient

Two additional fuzzy rules, ranging from $0 \%$ to $20 \%$, were set using ascending and descending fuzzy functions (see Section 8.1.2.2) to represent highly suitable and moderately suitable values respectively. In the highly suitable rule, the lower value was set to $0 \%$ while the upper value was set to $20 \%$. To indicate that membership should decrease from $0 \%$ to $20 \%$, the middle value is also set equal to the lower value $(0 \%)$. A similar approach was taken with the ascending moderately suitable rule, where the middle value was set equal to the upper value (20\%).

Although Boolean rules ranging from $0 \%$ to $10 \%$ and $10 \%$ to $20 \%$ could have been defined to represent the highly suitable and moderately suitable categories, the combined effect of the two fuzzy rules ensures a smooth transition between these categories. This is illustrated in Table 9-1 which lists the suitability values and levels for selected slope gradients. Values greater than 0 are used for factors (i.e. highly suitable, moderately suitable and marginally suitable), while values of less than 0 are used for constraints (i.e. permanently unsuitable and unsuitable at present). Refer to Section 8.1 for details of suitability value calculations. 
Table 9-1 Calculated suitability values and levels for selected slope values

\begin{tabular}{|c|c|c|}
\hline SLOPE (\%) & SUITABILITY VALUE & SUITABILITY LEVEL \\
\hline 0 & 3.0 & Highly suitable \\
\hline 5 & 2.75 & Highly suitable \\
\hline 10 & 2.5 & Highly suitable \\
\hline 15 & 2.25 & Moderately suitable \\
\hline 19.9 & 2.005 & Moderately suitable \\
\hline 20 & -2 & Permanently unsuitable \\
\hline
\end{tabular}

To view the spatial implications of the Slope gradient (\%) rules, a project was created in the projects page (see Figure 8-15). A project is essentially a virtual container that keeps record of the actions during land suitability mapping. This enables the storage of a user's activities so that a project can be reopened later. The mapping environment is opened by clicking on the newly created project in the projects table. When a new project is opened, a satellite image map of the Western Cape is presented (Figure 8-16). Because suitability analysis results are difficult to visualize at the scale of Figure 8-16 (i.e. 1:4 000 000), the mapping of the individual requirements is demonstrated on a smaller area of interest (AOI) in the greater Cape Town region (Figure 9-4). By using the zoom tool the scale and extent of the mapped area was changed to include Table Mountain (south of Cape Town) in the west and the Hottentots Holland Mountains (north-east of Somerset West) in the east.

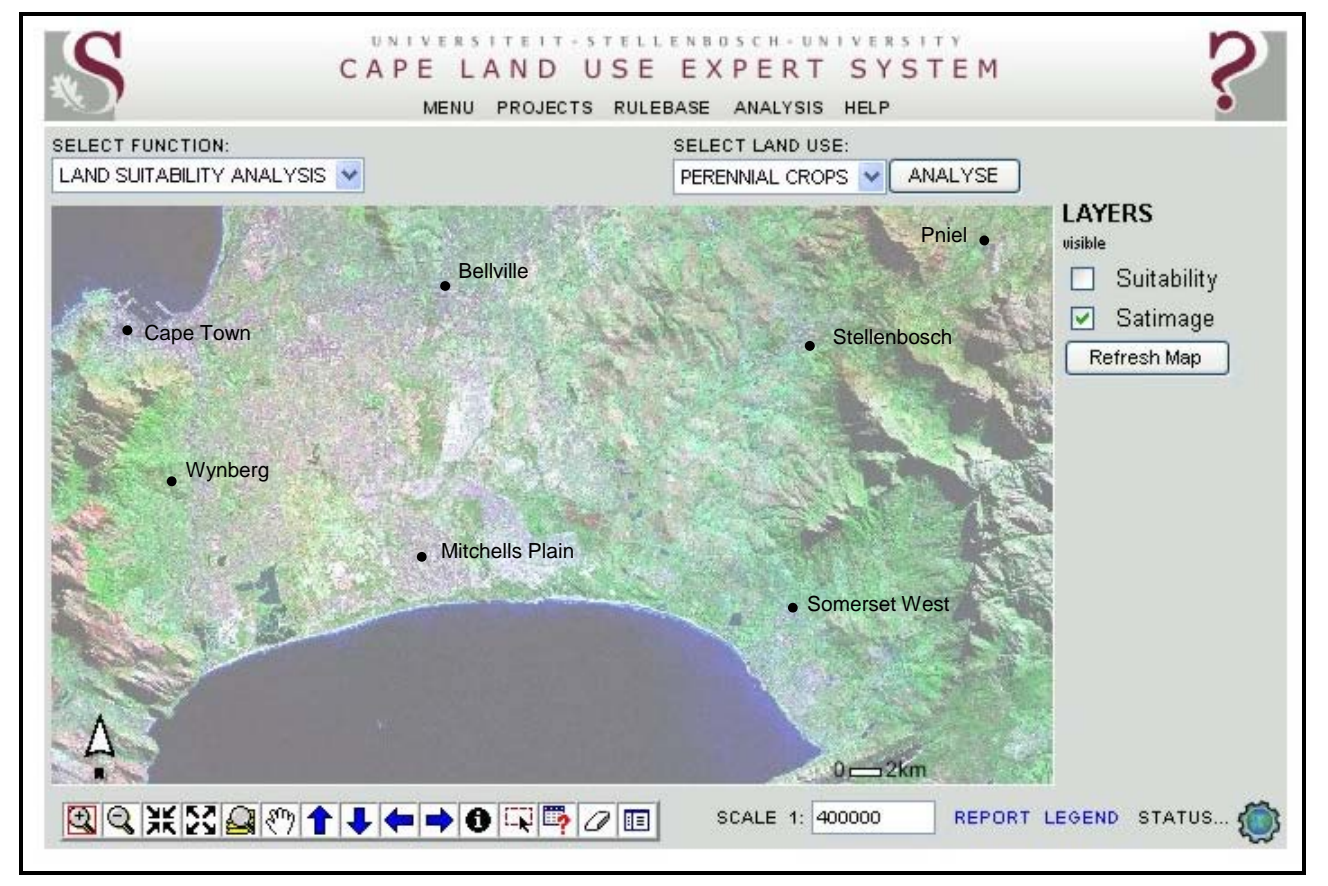

Figure 9-4 Satellite image of the AOI 
The suitability analysis for perennial crops based on the slope requirement was initiated by selecting Perennial Crops from the drop-down land use list in the menu frame above the map and by clicking on the ANALYSE button. The resulting slope requirement map (Figure 9-5) indicates that most of the AOI is highly suitable in terms of slope and that the mountainous areas were identified as being permanently unsuitable. Only a narrow band of hillslopes was classified as being moderately suitable.

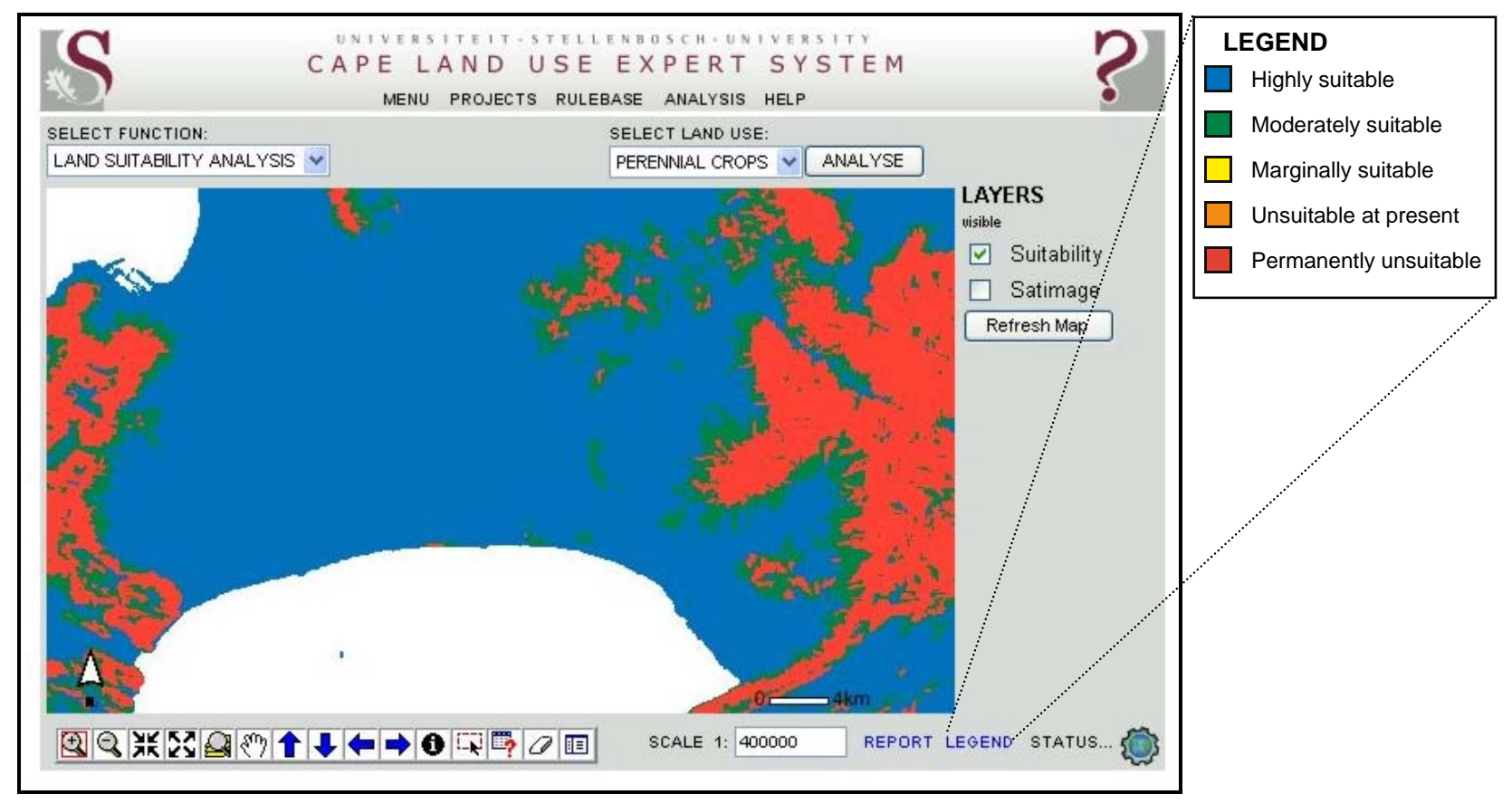

Figure 9-5 Slope requirement map of the AOI

The legend in Figure 9-5 is a key to the colours used for each suitability level. The standard legend is taken from a separate window that appears when the LEGEND link (bottom-right of map window) is opened and some categories may therefore be absent in a given analysis image.

For orientation purposes, the user can at any time toggle between the Suitability and Satimage layers in the layers list (to the right of the map) to enable or disable the display of the suitability result. If both the Suitability and Satimage layers are active, the suitability result overlays the satellite image, as shown in Figure 9-6.

\subsubsection{Aspect, curvature and elevation}

Although data for slope aspect, curvature and elevation is available in the land unit database, it was not used in the suitability analysis. More research is needed to determine the influence of these land properties on perennial crops. It is generally accepted that slope aspect, in combination with slope gradient, does play a role in the quality of perennial crops owing to its influence on the amount of sunlight an area receives (Carey 2005). In the southern hemisphere, moderately inclined northern slopes receive more solar radiation than southern or gently inclined 
slopes (Buckle 1996). Consequently, temperatures on northern slopes are generally higher where they may influence soil moisture and formation. In the relatively warm climate of the Western Cape, aspect has little impact on the minimum requirements of land for the establishment of perennial crops (Saayman 1981). However, slope aspect should be considered in suitability analyses of specific crops or cultivars (Carey 2005).

Concerning curvature, it is well known that day-night temperature differentials for land units in convex landscape positions are generally more stable and therefore less likely to be affected by frost (Buckle 1996). The soils on convex land components are dryer and better drained due to the divergence of runoff and groundwater flow from such areas. As with slope aspect, gradient has an indirect effect on crop suitability as it will affect soil formation over time. However, too little is known about the direct effects of curvature on perennial crops to set general suitability rules.

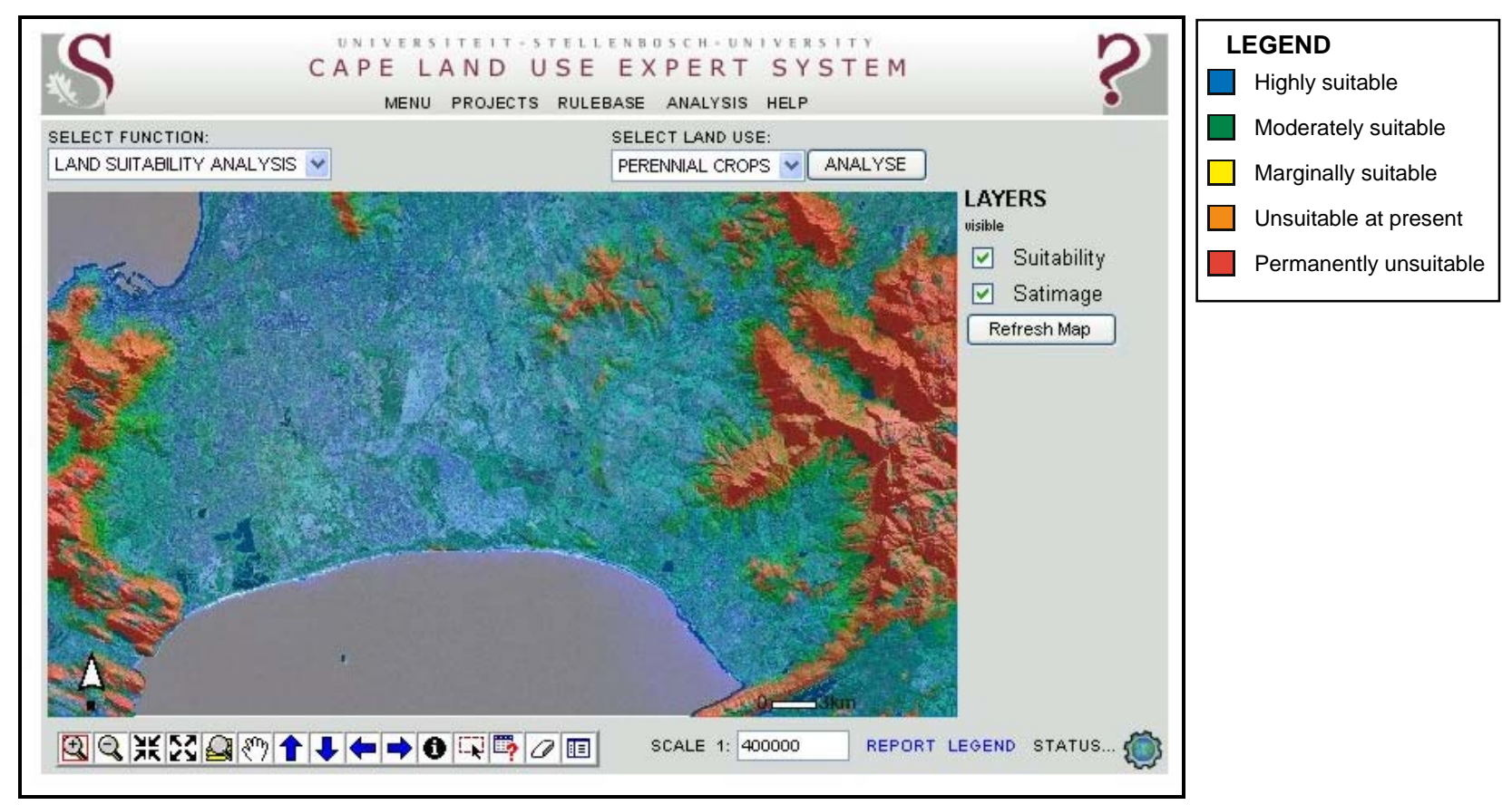

Figure 9-6 Suitability results overlaying a satellite image for orientation purposes

Elevation has a direct effect on climate because temperature decreases on average by about $0.3^{\circ} \mathrm{C}$ in South Africa with every $100 \mathrm{~m}$ above sea level (Saayman 1981). Land units on hills and mountains are therefore generally cooler than those in low-lying areas. No explicit rule to incorporate the effect of elevation on temperature was implemented in the rulebase because it is inherent in other land properties such as chill and heat units. The rules set for the climatic requirements of perennial crops are discussed next.

\subsubsection{Climate requirement rules}

Climate is the most important environmental variable affecting the production of fruit crops on a regional scale (Jackson 1999). Four climatic attributes relating to temperature and rainfall were 
considered in the suitability analysis of perennial crops. They are chill units, heat units, frost occurrence and mean annual rainfall.

\subsubsection{Chill units rules}

The cumulative number of hours that plants are exposed to temperatures ranging from $2.4^{\circ} \mathrm{C}$ to $9.1^{\circ} \mathrm{C}$ during winter is one of the most important climatic factors to consider for perennial crops. Most deciduous plants require a minimum number of chill units $(C U)$ - measured in the Western Cape as hours from leaf-drop in May until September - to satisfy dormancy, to stimulate growth, develop leaves, flower and set fruit. Failure to produce the required CU in areas with warm winters will prevent or reduce budding, resulting in poor crops (Reiger 2006; Schulze 1997). Most temperate fruit crops such as pome fruit (apples and pears) require at least 1000CU each winter (Reiger 2006), whereas peaches and apricots require between 806CU and 925CU (Valentini et al. 2004).

Due to the wide range of the chilling requirements of different varieties of perennial crops as well as the influence of microclimate on CU (Jackson 1999), no constraining chilling requirements were defined for CLUES. However, areas with less than 400 hours of chilling were considered marginally unsuitable for perennial crops, while land units with values of more than $1000 \mathrm{CU}$ were considered to be highly suitable for perennial crops. As in the case of slope gradient, fuzzy functions were used to facilitate gradual transitions between chill suitability levels (see Figure 9-7).

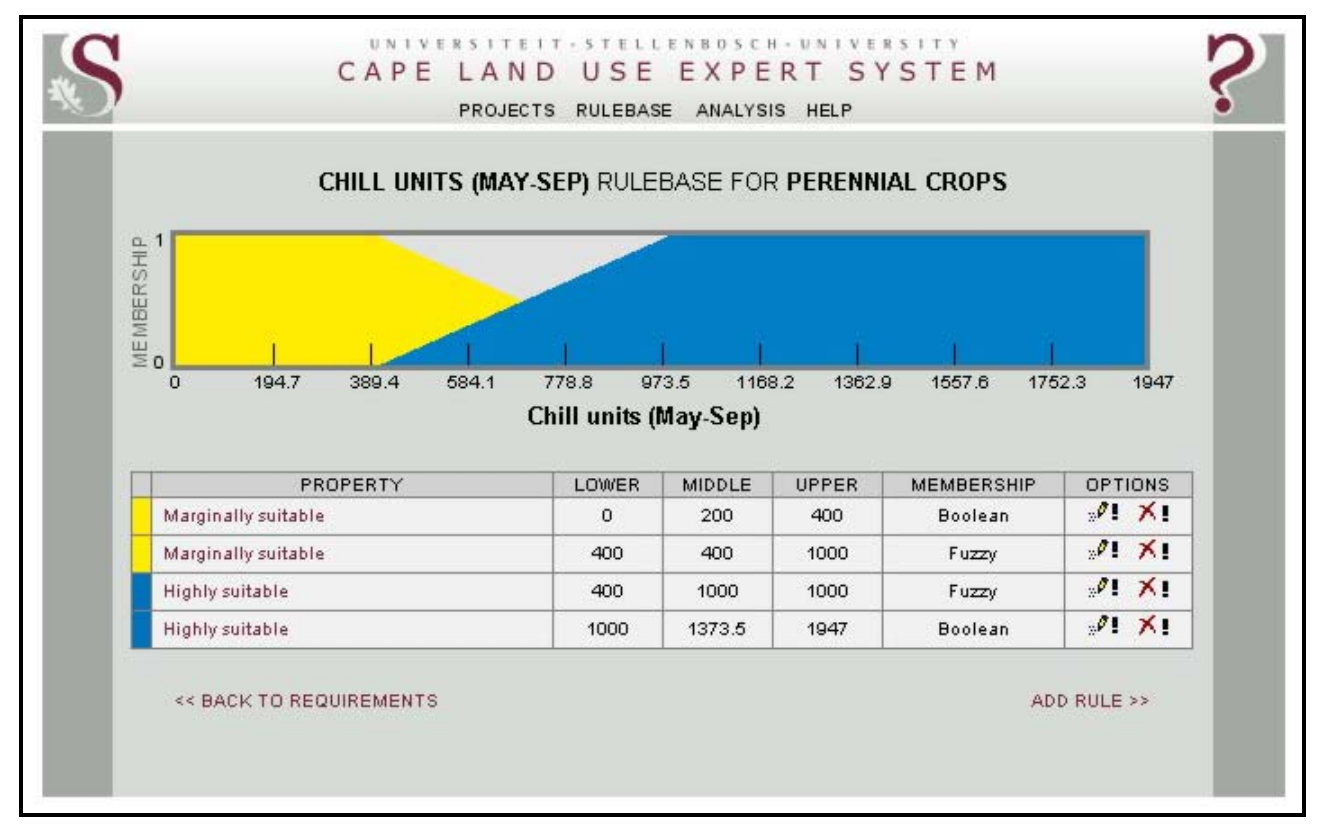

Figure 9-7 Chill units rules

Although no category was specified for moderately suitable, the overlapping nature of the two fuzzy rules, representing marginally suitable and highly suitable land units respectively, 
inherently encompasses this intermediate category. The inference engine allocates all land units with $\mathrm{CU}$ values ranging from 550 to 850 to this class. The spatial effect of the two fuzzy rules is illustrated in Figure 9-8.

It is clear from this requirement map that the chill factor has a strong relationship with elevation as most of the areas identified as being moderately or highly suitable are higher-lying areas, while the coastal and low-lying areas of the Cape Flats (i.e. around Mitchells Plain) are allocated to the marginally suitable category.

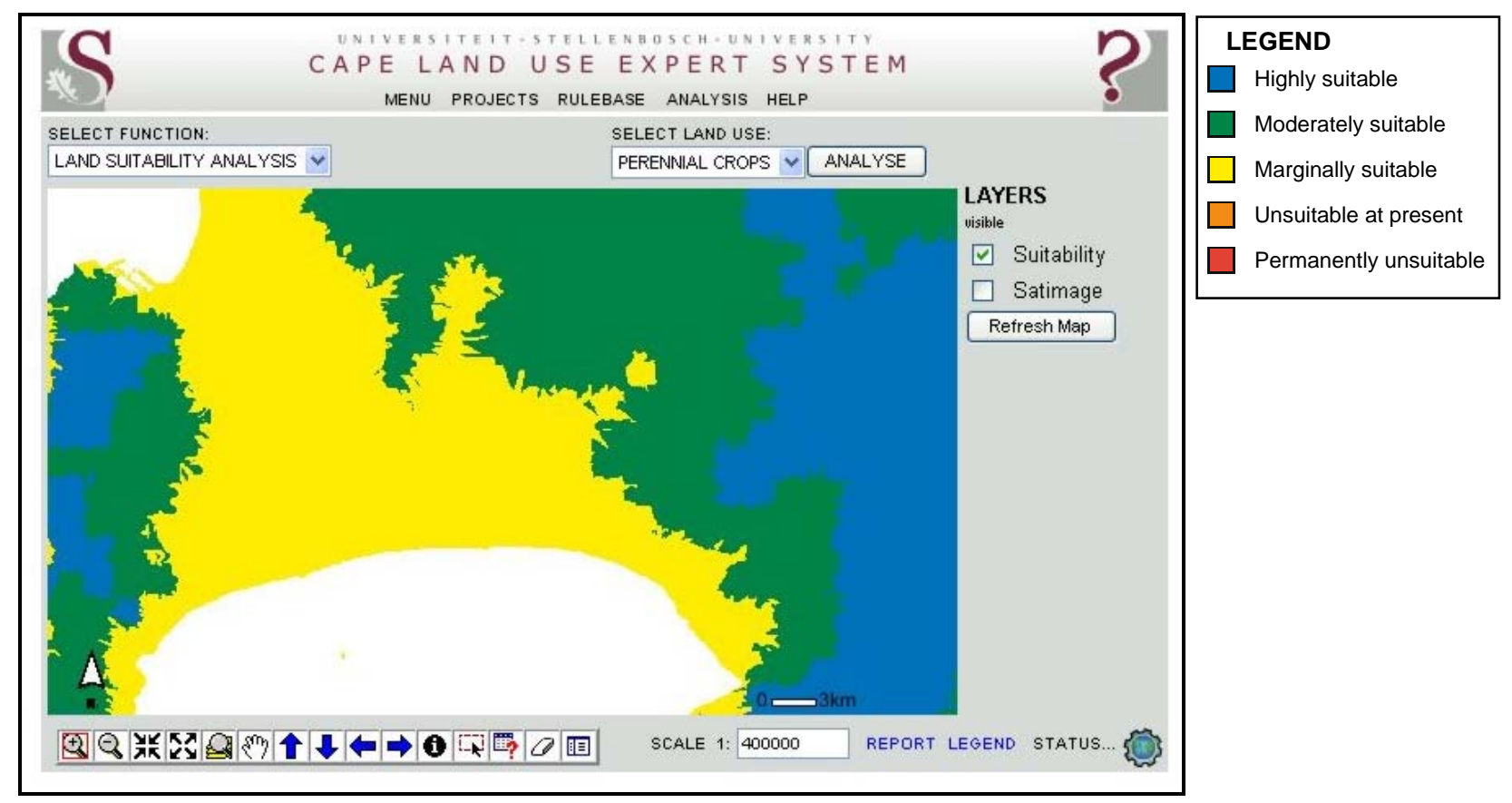

Figure 9-8 Chill units requirement map of the AOI

\subsubsection{Heat units rules}

Once the chilling requirement of a temperate woody plant has been satisfied, it must receive a certain number of heat units (HU) or growing degree-days (GDD) in order to resume growth. This is especially important for perennial crops as they require a minimum number of $\mathrm{HU}$ for fruits to ripen. Heat units are the number of days in a growing season (i.e. October to March in the Western Cape) with an average temperature of more than $10^{\circ} \mathrm{C}$ (Schulze 1997). HU are especially important for wine grapes which require a minimum of $1000 \mathrm{HU}$ to ripen, while areas with more than $2222 \mathrm{HU}$ are considered to be suitable for mass production of dessert wine cultivars (Saayman 1981). Some apple cultivars (Royal Gala) also require 1000HU (OrtegaFarias \& Leon 2002). Fruits such as some varieties of pears and apricots, require as few as 112HU (Valentini et al. 2004), while olives require about 150HU to flower (Orlandi et al. 2005).

In spite of the wide range of $\mathrm{HU}$ requirements of different perennial crops, it is generally accepted that land suitability for the production of perennial crops increases as HU increase and 
that areas with $\mathrm{HU}$ of more than 1000 will be suitable for all perennial crops. Two rules representing highly suitable were subsequently created for HU. The first uses a fuzzy function to affect an increase in membership to the highly suitable category for land units with HU values of 0 to 1000 and the second rule allocates full membership to all land units with HU values of more than 1000 (Figure 9-9).

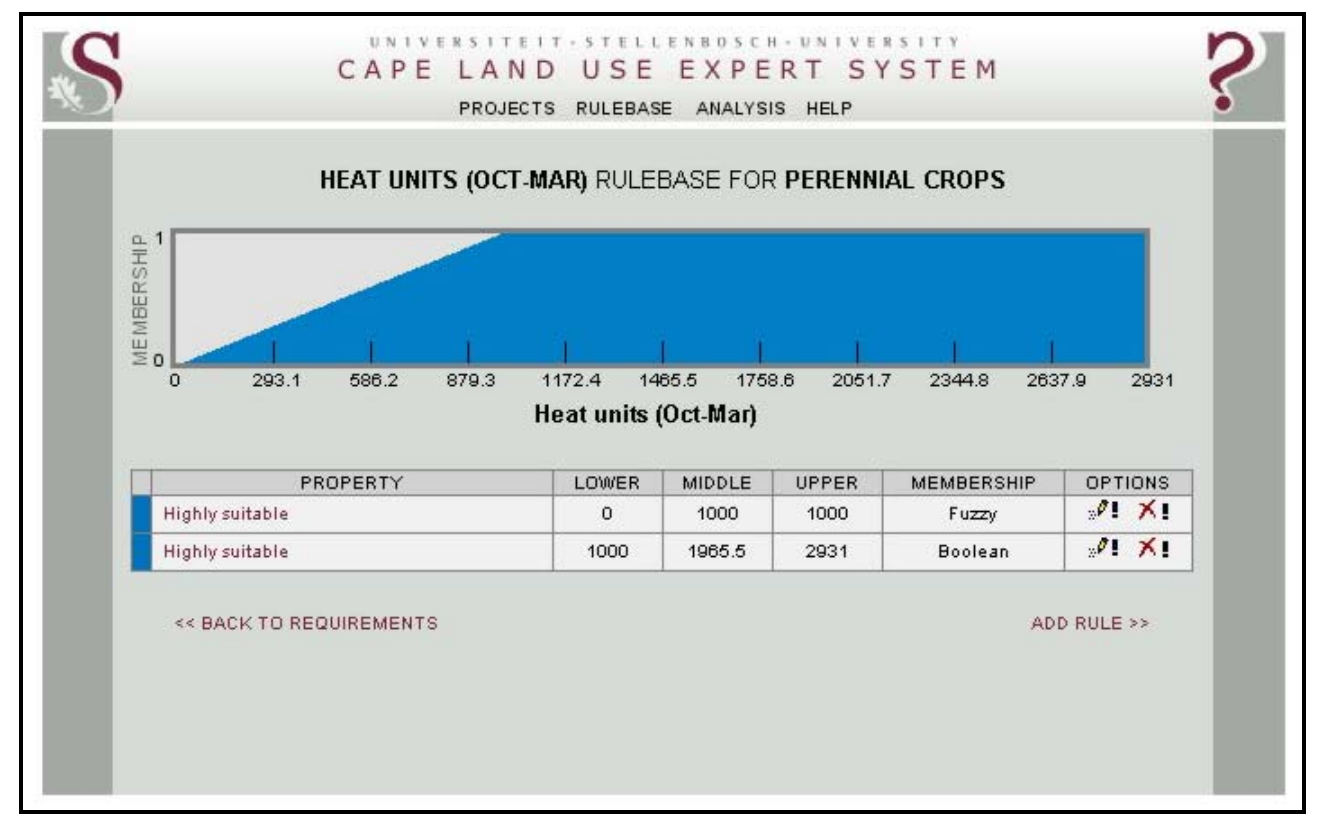

Figure 9-9 Heat units rules

The resulting requirement map is Figure 9-10 which, owing to the relatively high temperatures experienced in the Western Cape, clearly shows that not many land units will be negatively influenced by this requirement. In the AOI, only the mountain peaks (compare Figure 9-4) were rated as being moderately suitable for perennial crops while the rest of the AOI was rated as being highly suitable.

\subsubsection{Frost occurrence rules}

Most perennial crops can withstand very low temperatures (as low as $-18^{\circ} \mathrm{C}$ ) during their dormancy period (Reiger 2006) but they are less cold hardy during spring when leaf and flower buds begin to swell and bloom (Jackson 1999). Because temperatures in the Western Cape rarely fall below $5^{\circ} \mathrm{C}$, the main concern for perennial crops is temperatures below $0^{\circ} \mathrm{C}$ which expose buds and fruit to frost during the growing season. Areas prone to frost from September to March are therefore less suitable for perennial crop production. To identify such areas, the Frost (end) land property, indicating the date on which frost occurrence is likely to end (as averaged from historic data), was included in the analysis. Rules for marginally, moderately and highly suitable areas were defined as shown in Figure 9-11. 

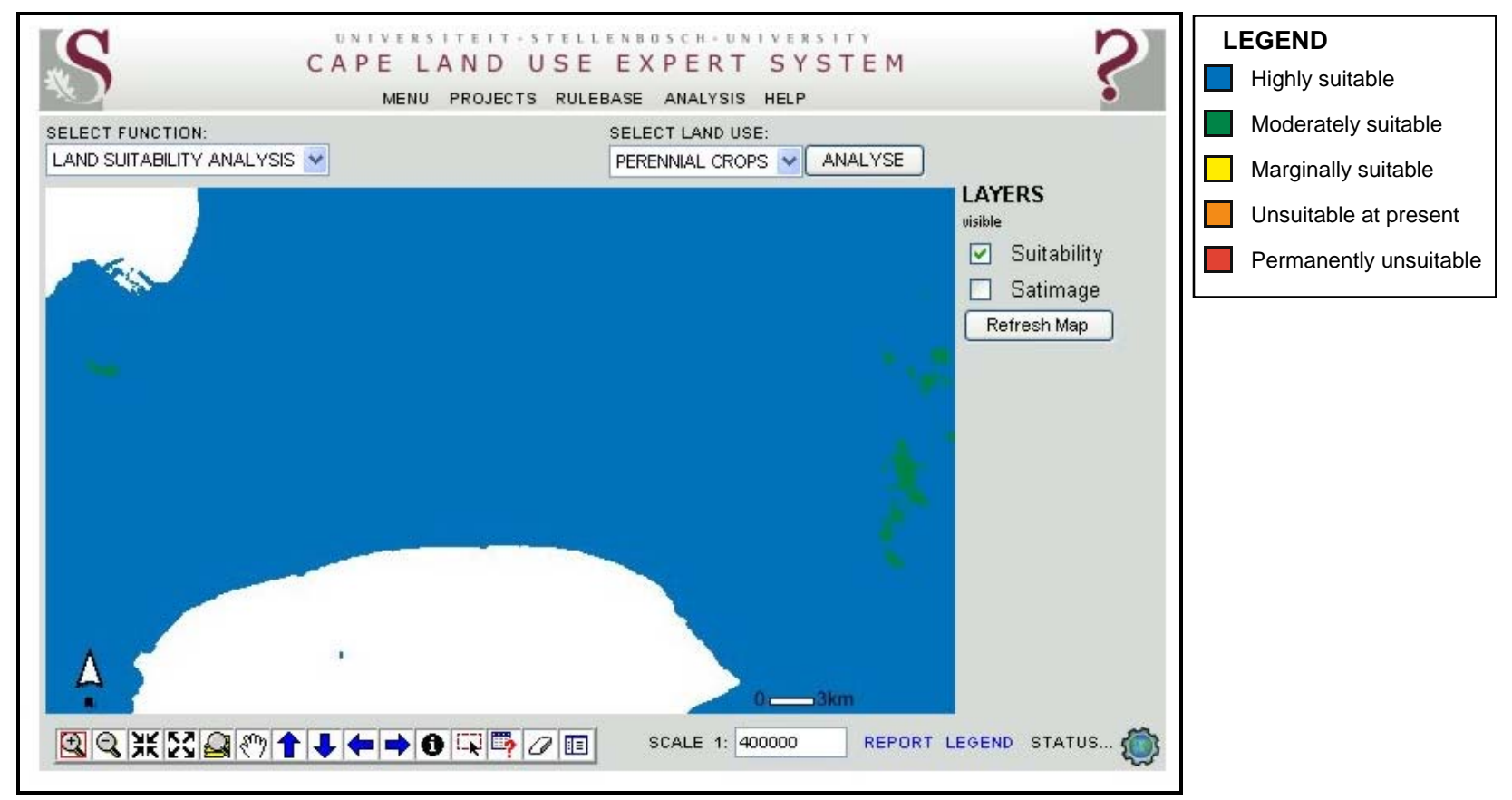

Figure 9-10 Heat units requirement map of the AOI

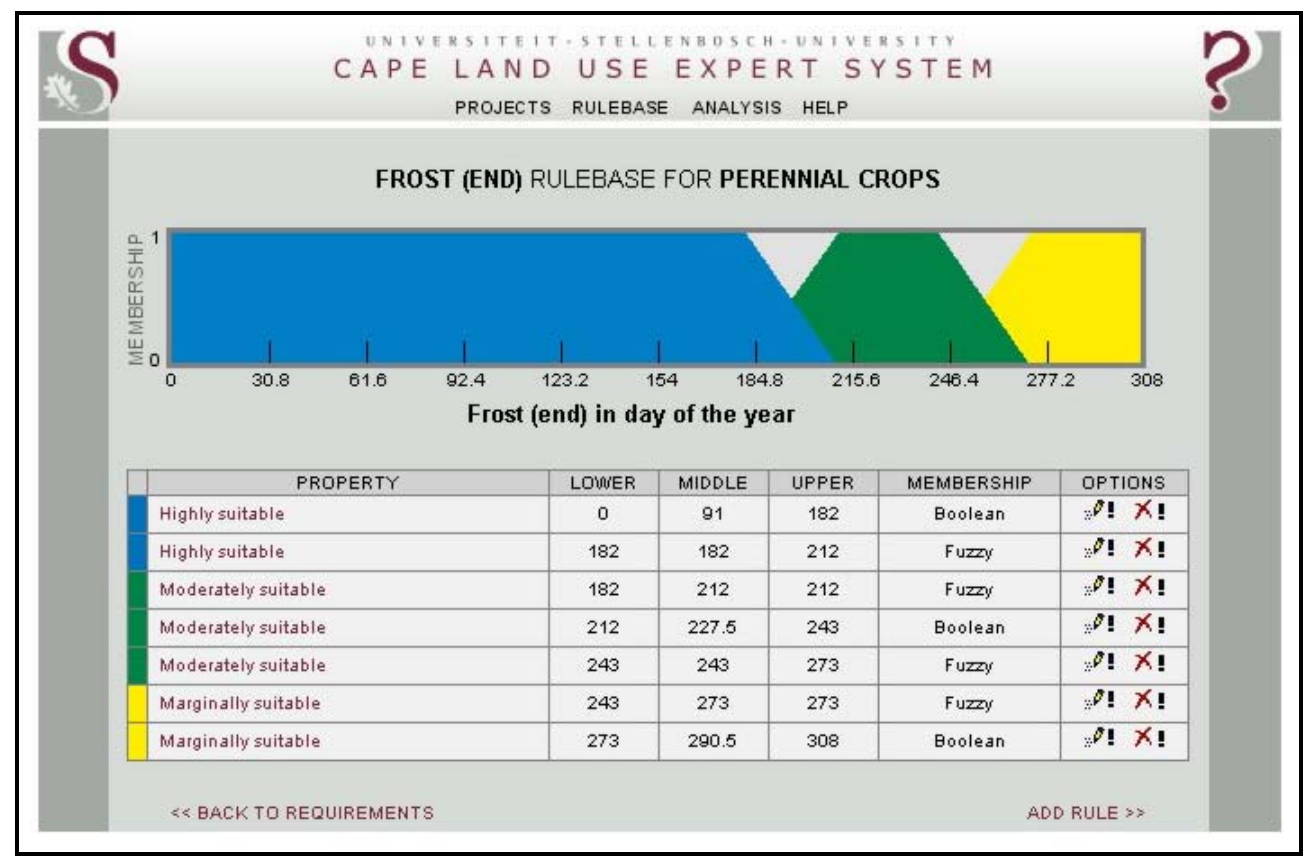

Figure 9-11 Frost requirement rules

Areas prone to late frost are considered to be marginally suitable. Because the frost (end) property is measured as days from the beginning of the year, all values of more than 273 (i.e. later than the end of September), are considered to be marginally suitable. Areas experiencing frost prior to the onset of the growing season (i.e. before day 212 or end of July), are considered highly suitable, while all other areas are considered to be moderately suitable for perennial crop production. Four additional fuzzy rules to create gradual transitions between the suitability categories have been formulated. 
The requirement map for frost (end) is shown in Figure 9-12. Due to proximity to the sea, most of the AOI is not adversely affected by frost and is consequently regarded as highly suitable. Only the Hottentots Holland Mountains north-east of Somerset West are mapped as being marginally suitable for perennial crop production.

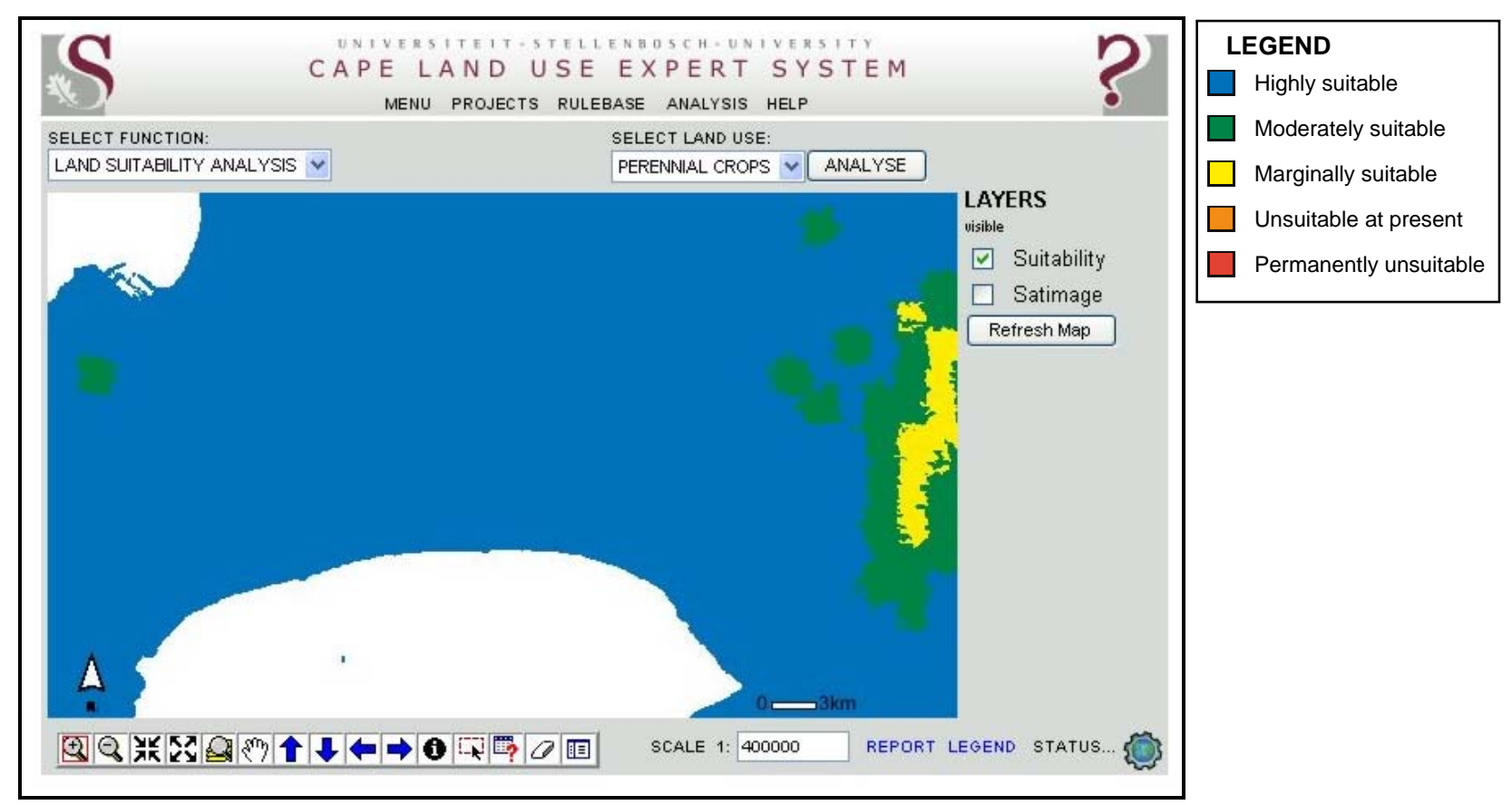

Figure 9-12 Frost requirement map of the AOI

\subsubsection{Rainfall rules}

Moisture availability is one of the main factors determining the growth performance of a plant. Moisture can be obtained from natural sources such as precipitation and soil, or it can be provided artificially through irrigation. In the Western Cape the amount of irrigation water needed to sustain optimal perennial crop growth is directly related to rainfall. To accommodate rainfall variations, seven rules relating to mean annual rainfall were added to the rulebase (see Figure 9-13).

Two fuzzy transitions were created in the ranges $150 \mathrm{~mm}$ to $400 \mathrm{~mm}$ and $600 \mathrm{~mm}$ to $850 \mathrm{~mm}$ to separate the marginally suitable, moderately suitable and highly suitable categories. Areas with an annual rainfall of more than $850 \mathrm{~mm}$ are considered to be highly suitable for perennial crops as only limited irrigation is likely to be required in such areas. The rules aim to indicate that irrigation requirements for perennial crops gradually increase as the annual rainfall decreases.

The annual rainfall requirement map (see Figure 9-14) shows that the entire AOI is either highly or moderately suitable for perennial crops. This can be expected because the AOI has a relatively high annual rainfall, particularly the mountains east of Stellenbosch where some of highest rainfall in South Africa is measured (more than $2000 \mathrm{~mm}$ annually). As a result, most the 


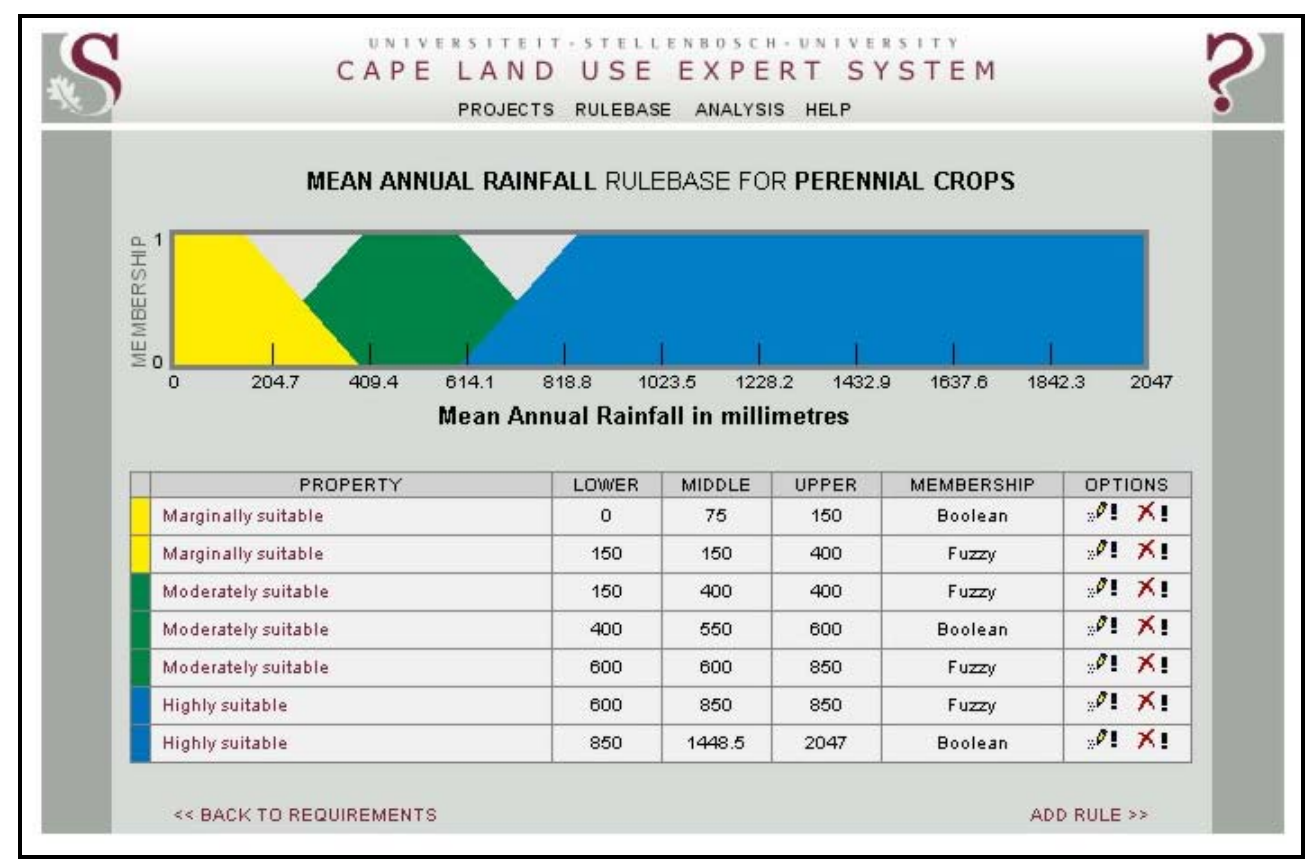

Figure 9-13 Mean annual rainfall requirement rules
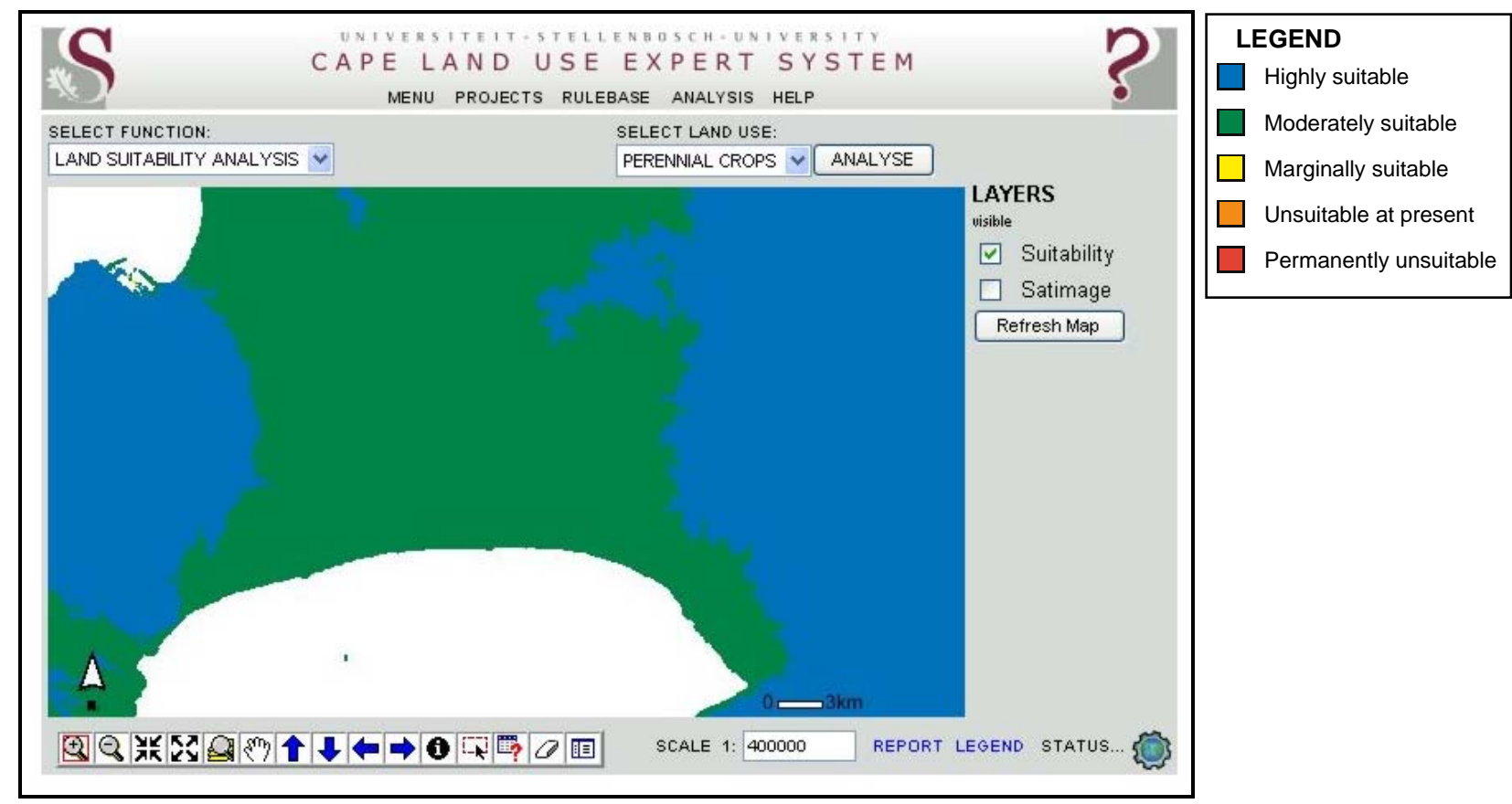

Figure 9-14 Mean annual rainfall requirement map of the AOI

vineyards and orchards in the Stellenbosch region are irrigated only during the driest months (January and February).

Apart from rainfall, the availability of water for plants is also affected by the moisture holding capacity of soils. The definition of soil requirements for perennial crops is described in the next section. 


\subsubsection{Soil requirement rules}

Soil properties are important for suitability analysis of perennial crops as they provide the necessary anchorage, moisture and nutrients for deciduous plants to develop. Two major attributes of soil are considered, namely clay content and effective depth.

\subsubsection{Soil clay content rules}

The clay content of soils determines to a great extent their moisture-holding capacity because clayey, fine-textured soils absorb more water than sandy, coarse-textured soils. Soils with excessive clay content can become waterlogged in wet areas thereby inhibiting plant growth. Growth can also be inhibited if there is too much fluctuation in soil moisture as is common in some areas in the Western Cape where very sandy soils occur (Saayman 1981). Finer-textured soils are generally also more fertile than sandy soils as they contain more organic matter and retain nutrients better. Concerning manageability, too clayey soils can be difficult to till and sandy soils which are more stable need frequent fertilization (Brown 2003; Lambrechts \& Ellis s.d.).

The influences of soil clay content in the A-horizon on the establishment of perennial crops were considered in defining the requirement rules (see Figure 9-15). Six fuzzy and two Boolean rules were defined. For the highly suitable category a Boolean rule was set at $10 \%$ to $20 \%$ and two asymmetrical fuzzy rules, ranging from $5 \%$ to $10 \%$ and $20 \%$ to $25 \%$ respectively, were created to implement a softer transition between the highly suitable and moderately suitable classes. A similar approach was taken for the transition between moderately suitable and marginally suitable. An additional Boolean rule was used to allocate values of more than $30 \%$ to the marginally suitable class.

The rules in Figure 9-15 were executed to produce the suitability map in Figure 9-16. By comparing the map with the rule set diagram in Figure 9-15, a good understanding of the soil clay content of the area can be deduced. Most of the areas rated as highly suitable for perennial crops in terms of clay content correspond well with the areas currently used for this land use: the lower slopes of the Table Mountain range and the winelands of Stellenbosch and Somerset West stand out. Most of the sandy Cape Flats are assigned to the moderately suitable class, while the high sandstone mountainous areas are classified as marginally unsuitable. 


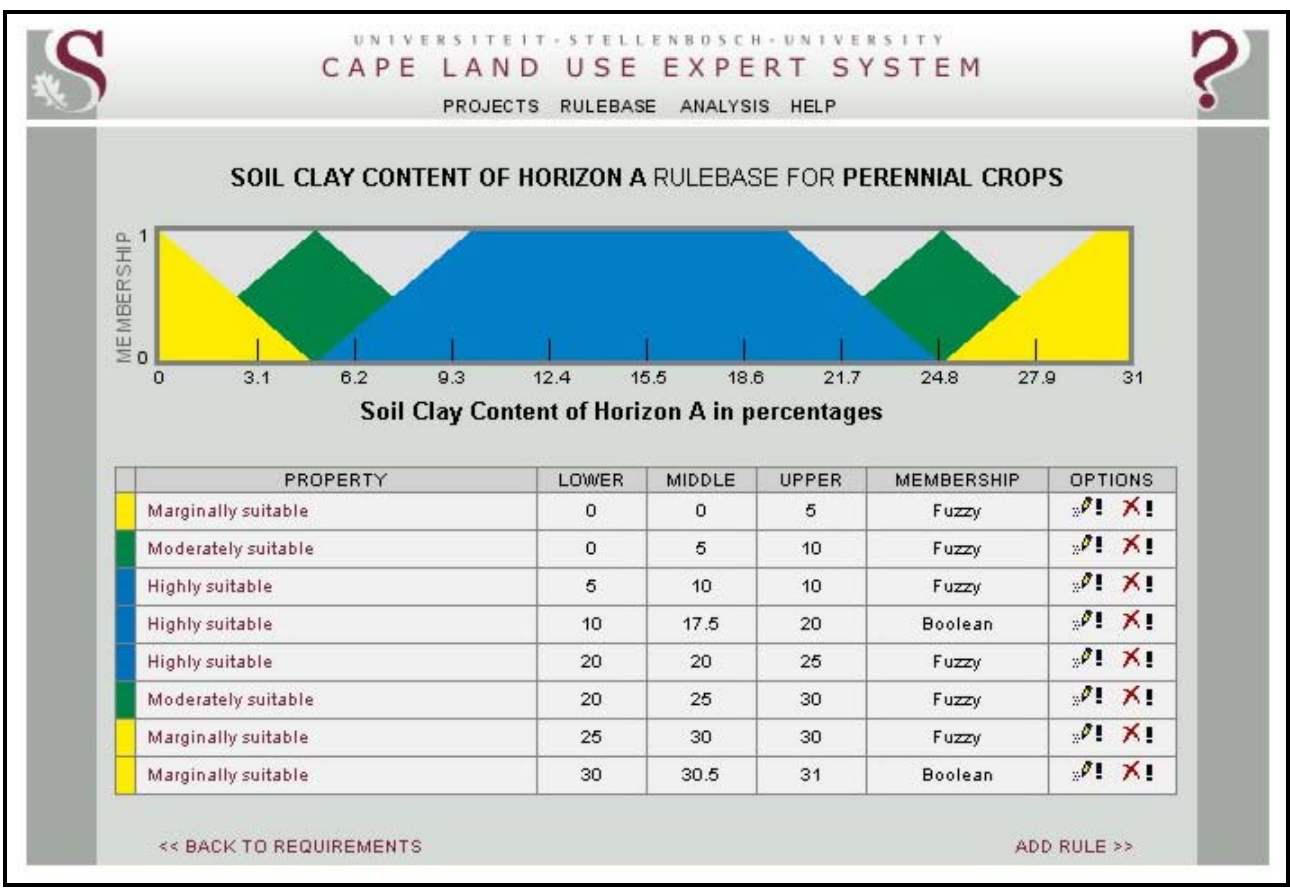

Figure 9-15 Soil clay content requirement rules
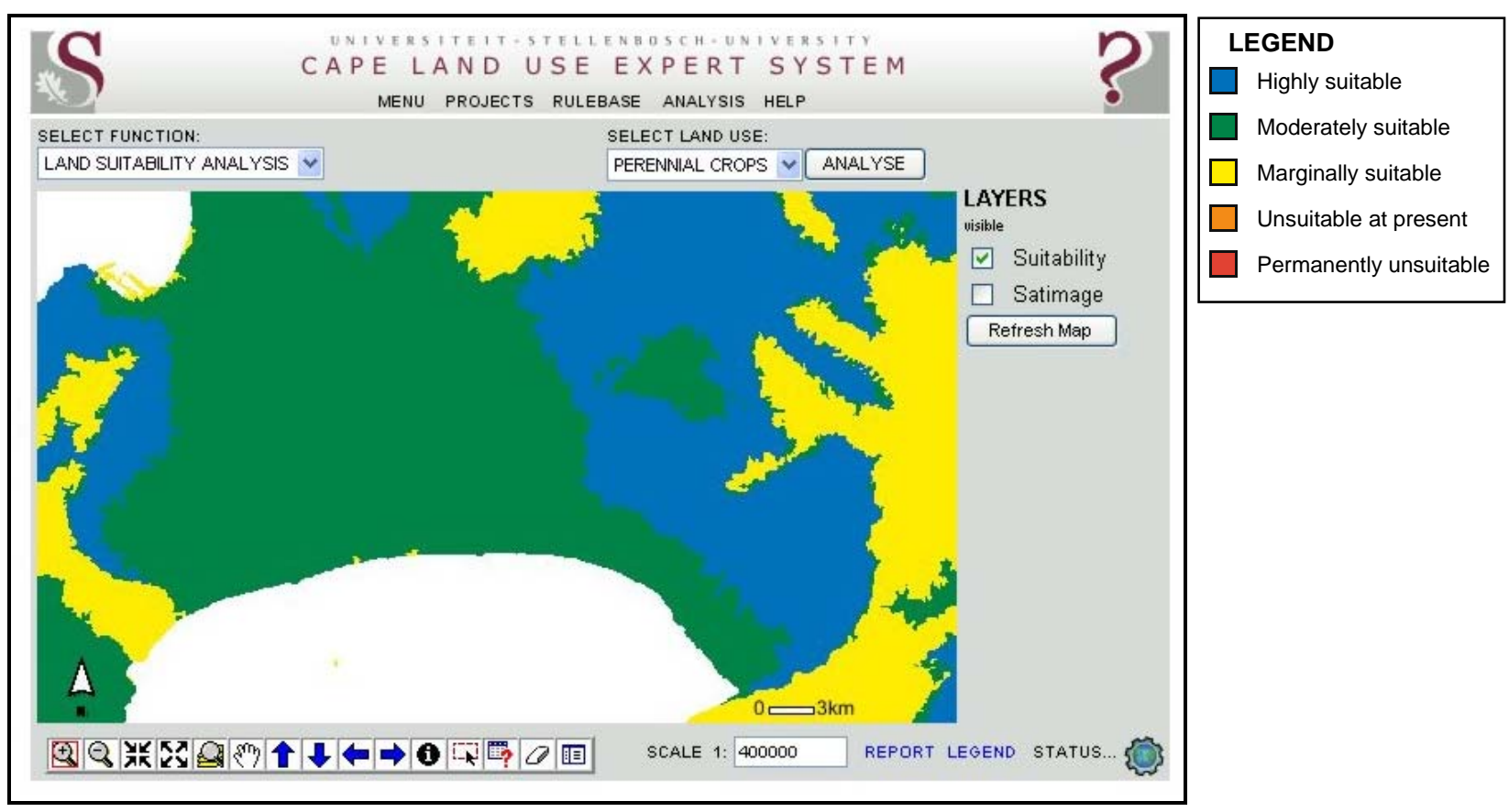

Figure 9-16 Soil clay content suitability map of the AOI

\subsubsection{Effective soil depth rules}

Effective soil depth, also called rooting depth, refers to the depth at which root penetration is strongly inhibited due to physical characteristics such as contact with bedrock, dense clay or permanent water, or due to contact with sub-soils with extreme chemical properties (Soil Survey Division Staff 1993). The effective depth of soil greatly determines the ability of soils to deliver adequate nutrients and moisture to perennial crops. It is generally accepted that deeper soils are 
more resistant to adverse climate conditions and that they ensure more stable growing circumstances (Saayman 1981).

As discussed in Section 5.2.2.2, the effective soil depth property was derived from the land type data which only differentiates between depths of $1200 \mathrm{~mm}$ or less. Soil series with effective depths of more than $1200 \mathrm{~mm}$ were simply indicated as being $1200 \mathrm{~mm}$ or deeper. In such cases the lower limit (i.e. 1200mm) was used. Due to the effect of averaging, most land units with deep soils were calculated to be shallower than they truly are. The values in the effective depth land property must therefore be interpreted as an index of depth rather than an absolute measure of effective depth. For instance, soils with an effective depth of more than $1000 \mathrm{~mm}$ should be interpreted as being $1000 \mathrm{~mm}$ or deeper.

Seven rules were defined for the effective soil depth property (Figure 9-17). In the first rule, shallow soils $(0 \mathrm{~mm}$ to $200 \mathrm{~mm})$ are allocated to the marginally suitable category. Soils with and effective depth of between $400 \mathrm{~mm}$ and $500 \mathrm{~mm}$ are considered to be moderately suitable for annual crop production, while deep soils (i.e. deeper than $700 \mathrm{~mm}$ ) are regarded to be highly suitable. Four fuzzy rules were defined to create transitions between the three Boolean rules.

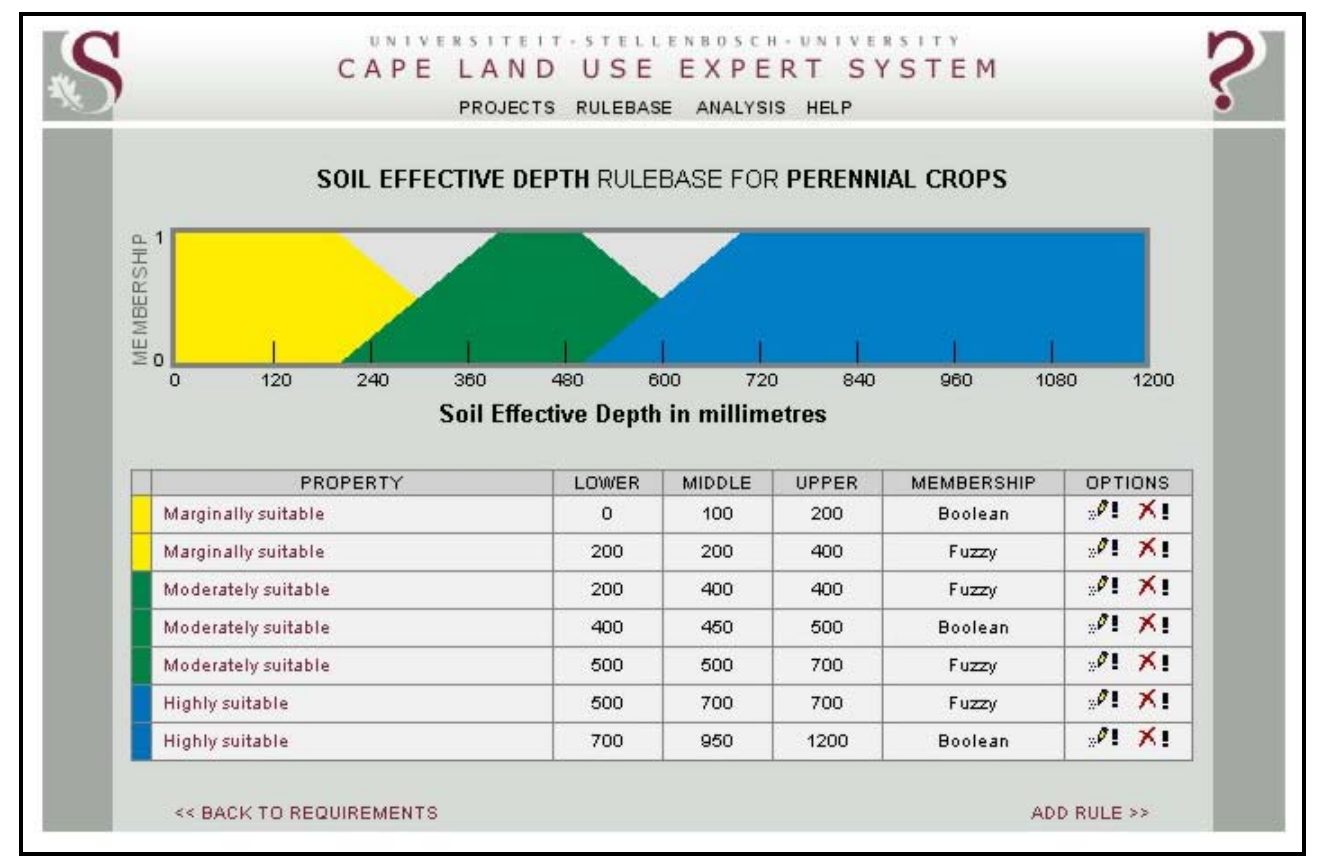

Figure 9-17 Effective soil depth requirement rules

The requirement map for perennial crops in terms of effective soil depth is shown in Figure 9-18. As expected, the more suitable deeper soils occur in the lower-lying areas. The mountainous areas are generally not considered to be suitable for annual crop production because much of the topsoil has been removed through erosion, the residual soil being too shallow for vineyard or orchard plantings. 

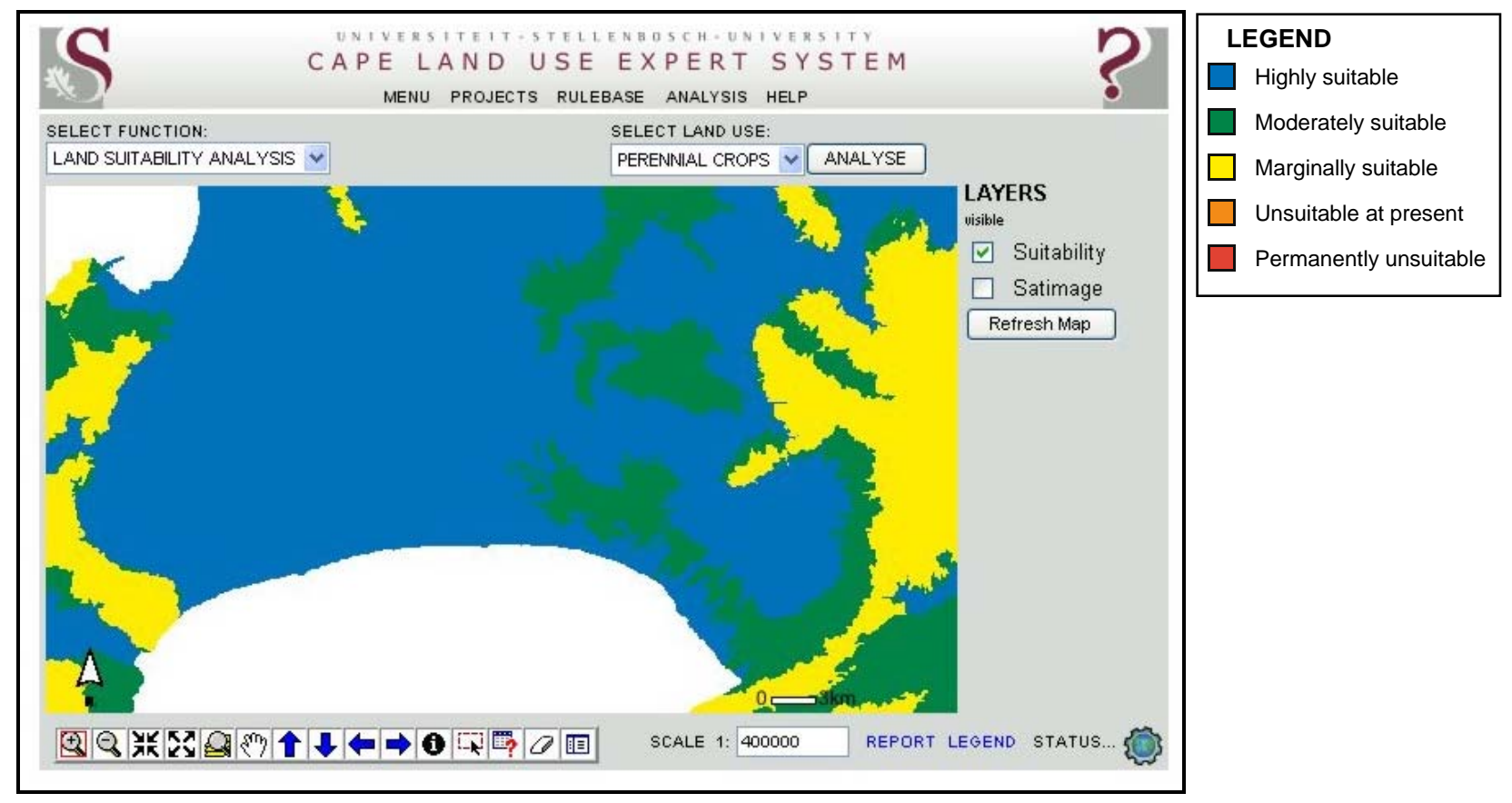

Figure 9-18 Effective soil depth requirement map of the AOI

\subsubsection{Current land uses and wetlands requirement rules}

The factors defined in the previous section relate to the natural and physical properties of land and do not take current land uses into account. To demonstrate how the present land uses can be incorporated into suitability analysis, two major land uses, namely urban and conservation areas, were used to define general constraints on agricultural development. Although wetlands is not a land use, it is added to the rulebase as a constraint to demonstrate how sensitive environmental areas can be included in a suitability analysis.

\subsubsection{Urban areas rules}

It was assumed that perennial crops cannot be established on land already transformed by urban use. Consequently, urban areas are considered to be permanently unsuitable for perennial crops. To implement this constraint, the Urban areas (distance to) land property was used to set a rule that excludes land units that are within $500 \mathrm{~m}$ from existing urban areas. A 500m buffer was used around urban areas to incorporate possible future expansion of the urban edge. Figure 9-19 shows the spatial effects when this rule applies.

\subsubsection{Conservation areas rules}

Conservation areas are protected by law from any agricultural development. National parks and nature reserves are therefore included as a constraint in the rulebase by using the Conservation areas (distance to) land property. As with Urban areas (distance to), all land units that are within 500 metres of conservation areas were excluded from further analysis. The land units 

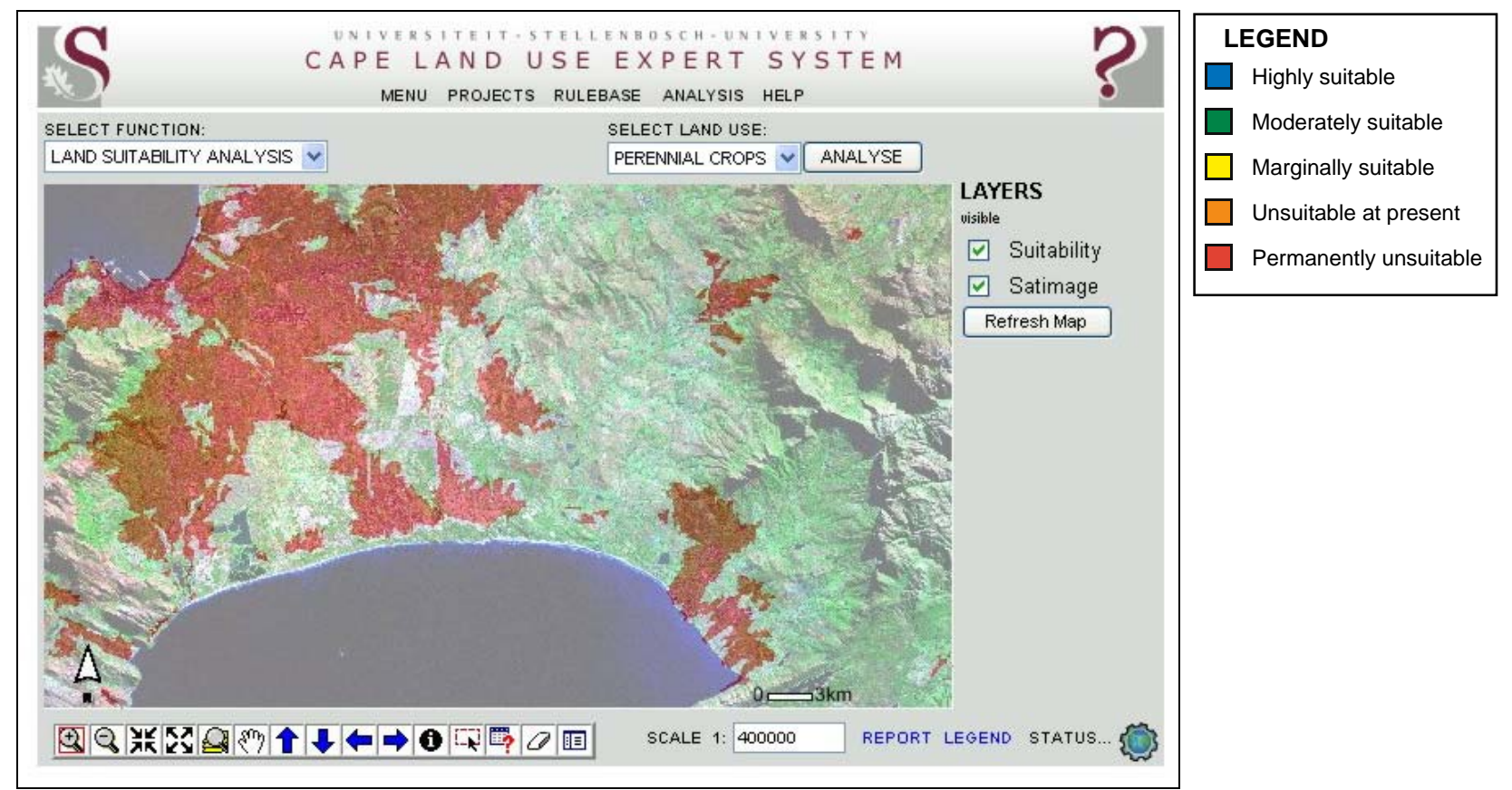

Figure 9-19 Urban areas in the AOI considered permanently unsuitable

affected by this constraint are shown in Figure 9-20 to be permanently unsuitable for perennial crop production.

\subsubsection{Wetlands rules}

Wetlands are among the most threatened ecosystems in the Western Cape and they must be protected at all costs from agricultural development (South Africa 1997). To exclude wetlands from consideration for perennial crops, a similar approach to the previous two agricultural constraints was taken using the wetlands (distance to) land property. Figure 9-21 shows the land units in the AOI that are affected by this requirement. Unfortunately, it is clear from the map that only major wetlands are included in the land unit database and that it should be expanded to include other smaller, but equally sensitive wetlands. A possible remedy is to use the information in the national wetlands map currently being developed by the South African National Biodiversity Institute (Dini 2007).

The constraints on perennial crop production discussed in this section do not constitute an exhaustive list. Other land cover and land uses such as water bodies, natural vegetation and mines, could also be included to reduce the number of land units considered for perennial crops. There is also a range of other factors, such as soil $\mathrm{pH}$, salinity and access to irrigation water, that may contribute to the suitability of land for perennial crops. Nevertheless, the factors discussed in this section should suffice to demonstrate the abilities of CLUES. 

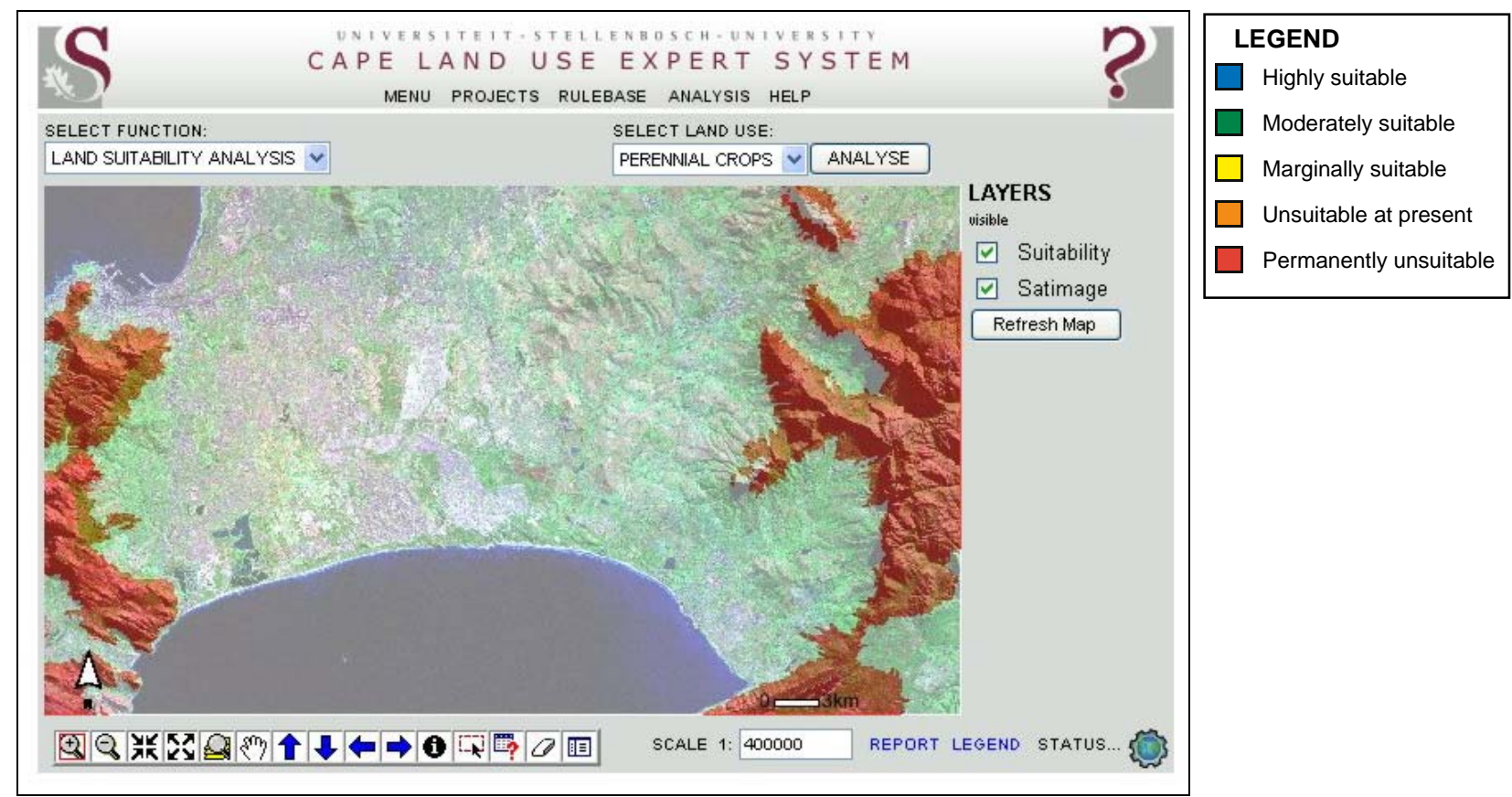

Figure 9-20 Conservation areas in the AOI considered permanently unsuitable

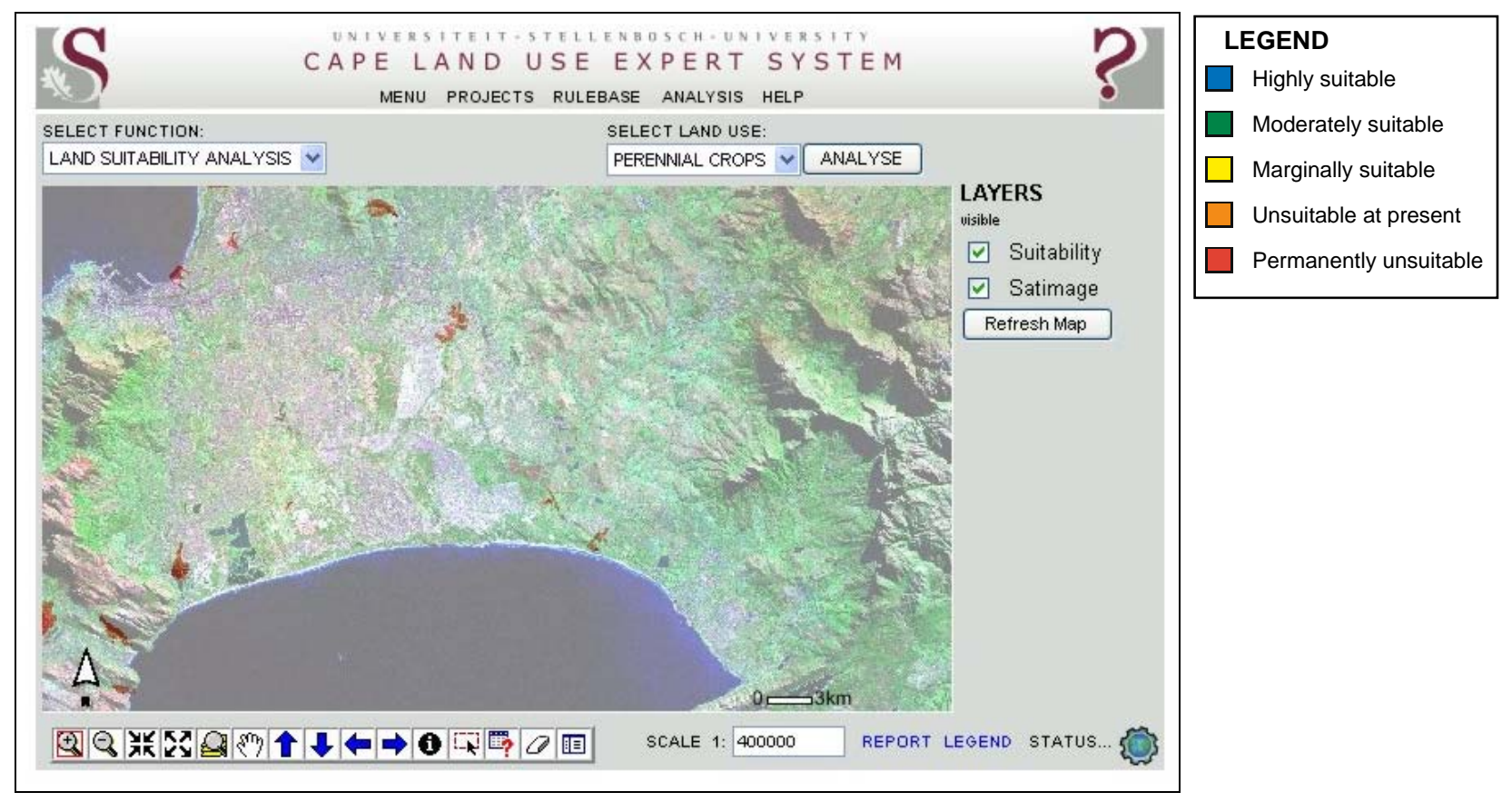

Figure 9-21 Wetlands in the AOI considered permanently unsuitable

\subsection{WEIGHTING SUITABILITY FACTORS}

All the factors defined above for the suitability analysis of land for perennial crop production are not equally important for determining suitability. The CLUES inference engine is designed to incorporate differences in importance by means of a weighting scheme. The weights assigned to the individual requirements were obtained by using the analytical hierarchy process (AHP) (see Section 2.3.1.5). The resulting AHP comparison matrix is shown in Table 9-2 (refer to Table 2-1 for scale value descriptions). 
Table 9-2 AHP comparison matrix of weights assigned to land use requirements

\begin{tabular}{|c|c|c|c|c|c|c|c|}
\hline & 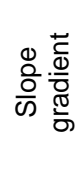 & 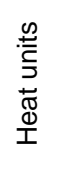 & 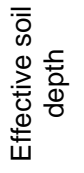 & 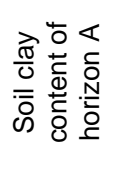 & 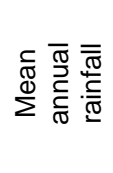 & 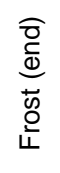 & 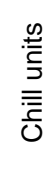 \\
\hline $\begin{array}{l}\text { Slope } \\
\text { gradient }\end{array}$ & - & $1 / 2$ & $1 / 4$ & $1 / 4$ & 1 & $1 / 4$ & $1 / 4$ \\
\hline Heat units & 2 & - & $1 / 2$ & $1 / 2$ & 2 & $1 / 2$ & $1 / 2$ \\
\hline $\begin{array}{l}\text { Effective soil } \\
\text { depth }\end{array}$ & 4 & 2 & - & 1 & 4 & 1 & 1 \\
\hline $\begin{array}{l}\text { Soil clay } \\
\text { content of } \\
\text { horizon A }\end{array}$ & 4 & 2 & 1 & - & 4 & 1 & 1 \\
\hline $\begin{array}{l}\text { Mean annual } \\
\text { rainfall }\end{array}$ & 1 & $1 / 2$ & $1 / 4$ & $1 / 4$ & - & $1 / 4$ & $1 / 4$ \\
\hline Frost (end) & 4 & 2 & 1 & 1 & 4 & - & 1 \\
\hline Chill units & 4 & 2 & 1 & 1 & 4 & 1 & - \\
\hline
\end{tabular}

An online AHP application developed by the Canadian Conservation Institute (2005) was used to calculate the eigenvectors from the comparison matrix to determine the overall importance of each factor. The resulting weight (importance) values are expressed as percentages in Figure 9-22. Although very little information about the relative importance of each of the factors could be found in the literature, most sources emphasize the importance of chill units during dormancy, especially for pome fruit. Frost during the growing season was considered to be critical for most perennial crops. These two factors, along with the requirements related to soil, consequently received the highest weightings (20\% each). The remaining $20 \%$ were allocated to heat units (10\%), slope gradient (5\%), mean annual rainfall (5\%), urban areas $(0.01 \%)$, wetlands $(0.01 \%)$ and conservation areas $(0.01 \%)$. Owing to the Western Cape's relatively warm climate, heat units are generally less important than chill units, while slope gradient is considered to be slightly more important than mean annual rainfall as it is assumed that irrigation infrastructure is available. 


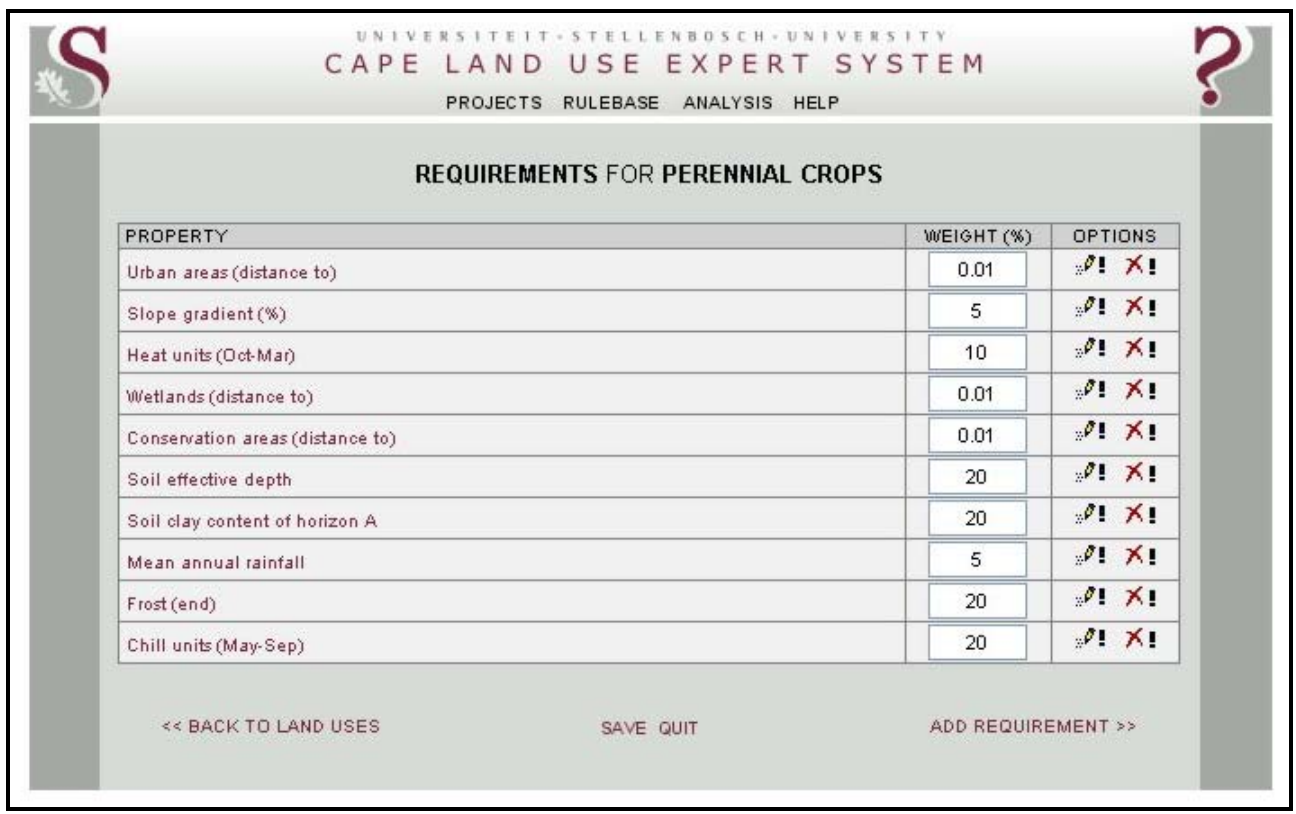

Figure 9-22 List of perennial crop requirements with weights shown on the req page

The three constraints, namely urban areas, wetlands, and conservation areas, were given weights of $0.01 \%$ each. Because requirements with weights of 0 are ignored by the inference engine, low values of slightly more than zero should be used to activate the constraints and to indicate that they do not inordinately contribute to or detract from the resulting suitability values. This approach to incorporating constraints is necessary because a requirement can act as a constraint (i.e. only consist of permanently unsuitable and unsuitable at present rules) or a factor (i.e. include marginally, moderately or highly suitable rules). A requirement, such as the slope gradient requirement in Figure 9-3, can however act as a constraint and a factor if it includes rules of both types. A weight of 0.01 is used for constraints to indicate that a requirement should not be interpreted as a factor. This ensures that land units that are permanently unsuitable in terms of any of the land requirements are not affected by other factors, no matter how well they perform.

It is important to note that the above weighting scheme is by no means a definitive solution for perennial crops as many horticulturalists will quite likely disagree with the chosen weights. This weighting scheme was merely created to demonstrate the functionality of the system. Ideally, more realistic weights and criteria should be derived from input obtained in a series of interviews with experts because the best solutions are often obtained when a group of experts participates in the AHP process.

\subsection{CASE STUDY SUITABILITY ANALYSIS AT VARYING SCALES}

The final step in the suitability analysis procedure is the production of suitability maps. The following sections demonstrate how suitability maps can be created for different areas and at 
varying levels of detail (i.e. map scales) to support land use decisions. Four case studies done for areas of decreasing size and increasing map scale are reported, namely greater Cape Town, Swartland, rural Malmesbury and Stellenbosch.

\subsubsection{Greater Cape Town}

The suitability analysis for perennial crops was done using the land use requirements and weights set out in the previous two sections. The analysis and mapping procedures took less than two minutes to execute for this AOI. Figure 9-23 is the resulting suitability map for perennial crop production in greater Cape Town. Most of the land units in this AOI are allocated to the permanently unsuitable suitability level. These units comprise urban and conservation areas, wetlands and terrain with slope gradients of more than $20 \%$.
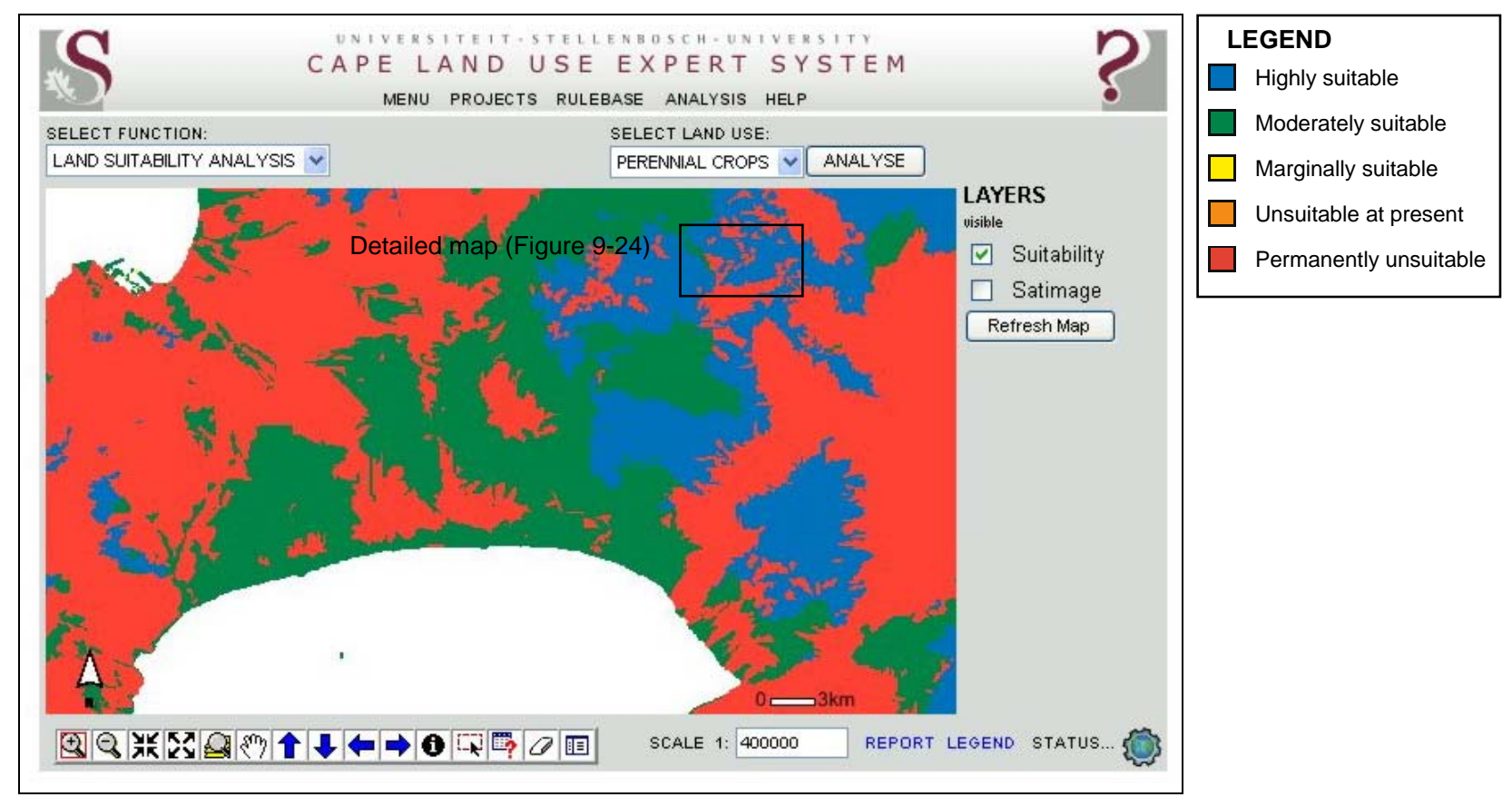

Figure 9-23 Suitability map for perennial crops in the greater Cape Town AOI

None of the land units in the AOI were identified as being marginally suitable for perennial crop production as this category is predominantly concealed by the permanently unsuitable class (compare Figures 9-8, 9-12, 9-16 and 9-18). This is especially noticeable in the Cape Flats area where most of the land units are marginally suitable in terms of chill units (see Figure 9-8). Land units in this area that were not masked by the permanently unsuitable category were upgraded to moderately suitable, mainly because they scored high in terms of heat units, frost end and effective soil depth.

\subsubsection{Stellenbosch}

The overall suitability of land for perennial crop production was calculated to be high in the Franschhoek-Stellenbosch-Somerset West region. This area scored high or moderate for most of 
the requirements and it coincides well with areas currently used for producing wine grapes. This is substantiated by a larger-scaled satellite image (Figure 9-24d) of the area north of Stellenbosch (location indicated as boxed in Figure 9-23) in which orchards and vineyards are clearly visible. By overlaying the suitability layer (Figure 9-24a) on the satellite image one can visually compare the suitability levels with current land uses. Observe that some areas currently under vineyards are identified as being unsuitable for perennial crops due to the $20 \%$ slope gradient. Some vineyards near to Stellenbosch are excluded according to the rule that land units within $500 \mathrm{~m}$ of urban areas are classified as being permanently unsuitable for perennial crops.

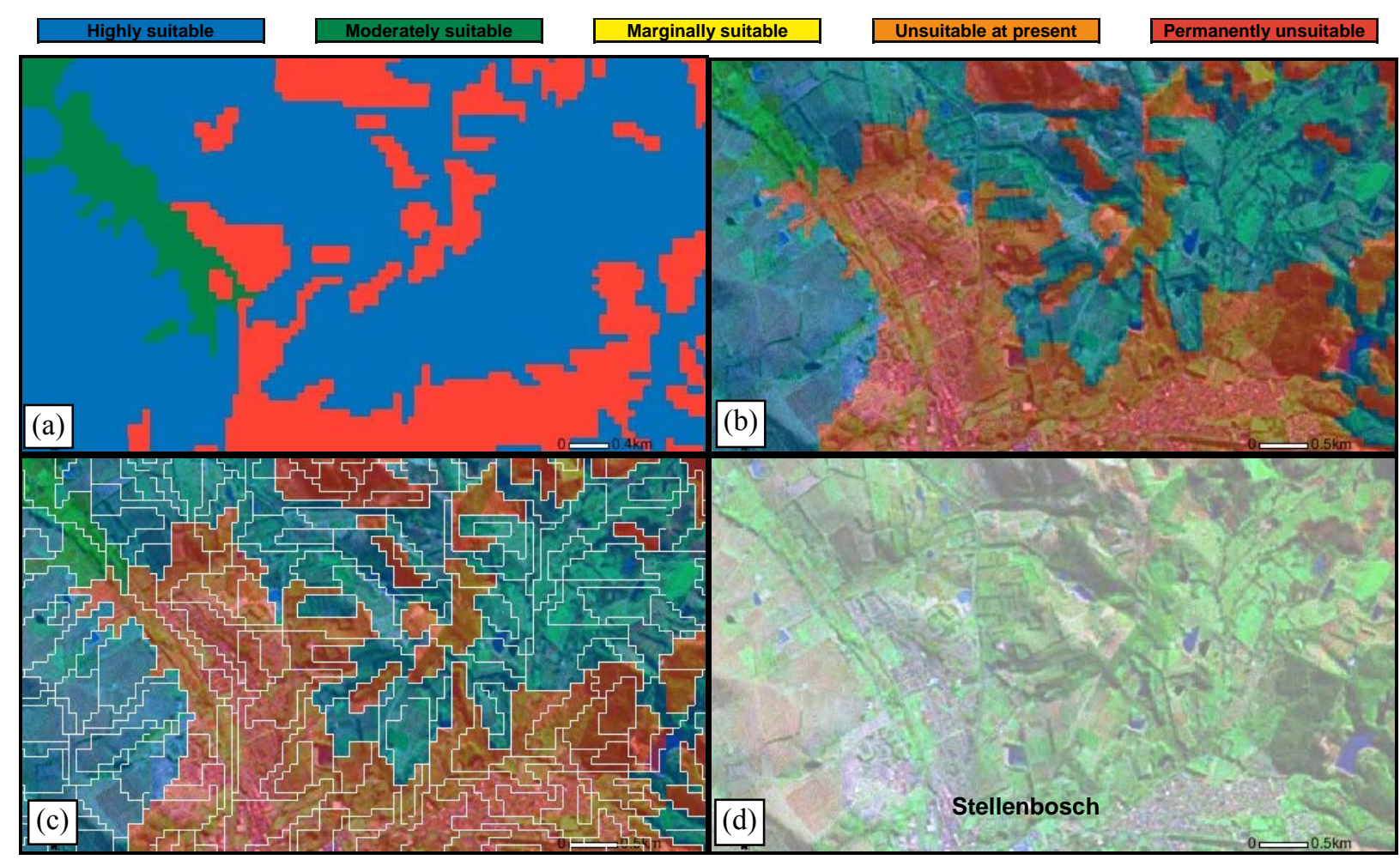

Figure 9-24 A compilation of CLUES screen captures showing detailed maps of (a) the suitability analysis result,

(b) the suitability overlay, (c) land unit outlines, and (d) the satellite image of the area north of Stellenbosch

Although the use of land components as mapping units is effective for natural environmental variables such as climate and soil, this example illustrates that they have limitations when used with features unrelated to terrain. For instance, urban edges often do not coincide with the boundaries of the land units (i.e. land components). This is strikingly apparent when the outlines of the land units are superimposed on the suitability map (Figure 9-24c). A possible solution to this limitation is to include boundaries of selected features in the land unit mapping process. But this will substantially increase the number of land units in the database, which in turn will negatively influence the system's overall response times. 
It is clear from Figure 9-24 that most of the land units identified in the Stellenbosch area as being highly suitable for perennial crops are indeed being used for this purpose. However, the aim of suitability mapping may also be to identify areas that are not being optimally or even illegally used so that alternative uses can be considered or remedies undertaken. To illustrate the further potential of suitability analysis, a proportion of the Swartland was chosen as an alternative AOI.

\subsubsection{Swartland}

The Swartland (Figure 9-25a) is a major wheat-producing area in the Western Cape where the recently improved irrigation infrastructure has fortuitously made it possible to introduce perennial crops.

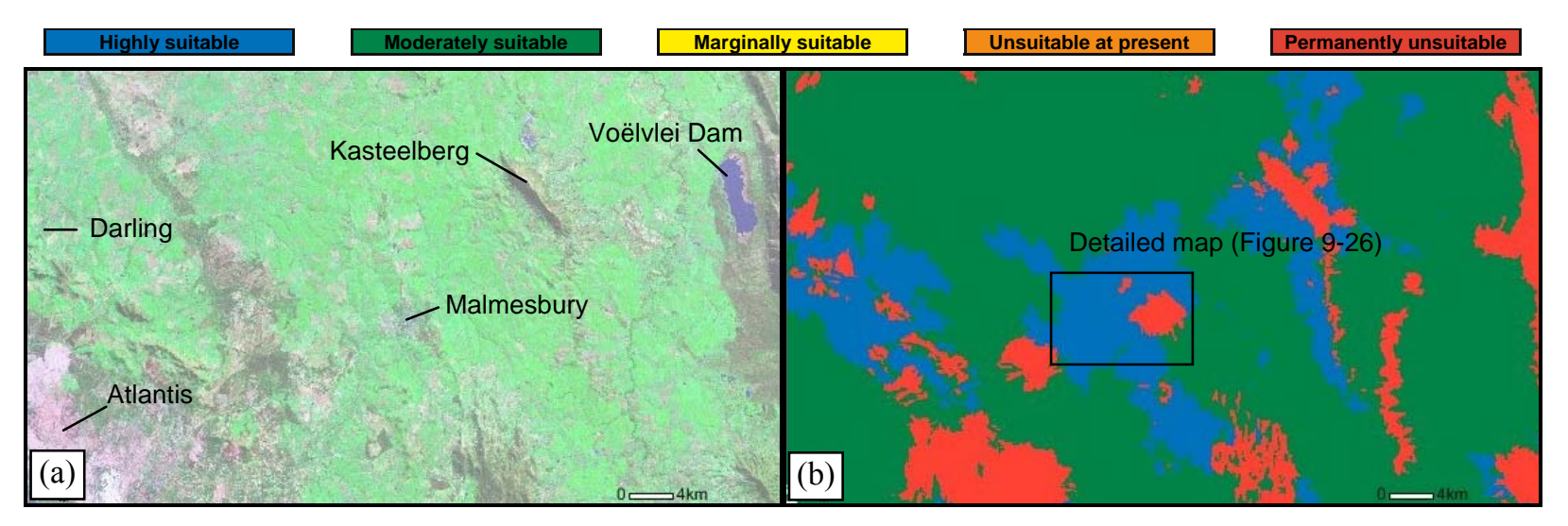

Figure 9-25 A compilation of CLUES screen captures showing (a) the satellite image and (b) the suitability analysis results for the Swartland area

According to the suitability analysis, most of the land units in the region are moderately suitable for perennial crops, while some areas around Darling and Malmesbury and the hillslopes south and north of Kasteelberg, are identified as highly suitable (Figure 9-25b). The land units excluded by the analysis are mostly those related to excessive slope and urban land use.

\subsubsection{Rural Malmesbury}

A larger-scale map of the rural area west of Malmesbury in the Swartland (Figure 9-26) reveals that the bulk of the land units that are highly suitable for perennial crops are currently being extensively worked for the cultivation of annual crops. Two areas are permanently unsuitable for perennial crops: the larger one was excluded mainly due its proximity to the town, while the small area in the north is a wetland (Figure 9-26b). As observed in Figure 9-24, some irregularities in suitability are related to the edges of urban areas. This anomaly is attributable to 


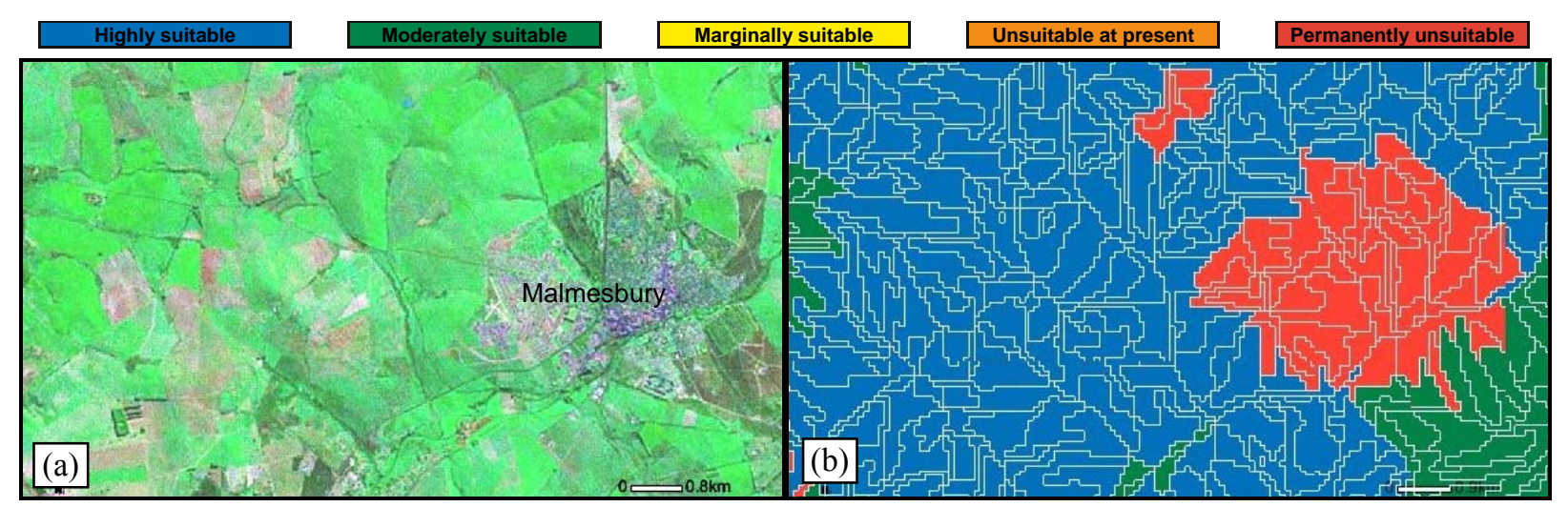

Figure 9-26 A compilation of CLUES screen captures showing detailed maps of (a) the satellite image and (b) the suitability analysis results for the area west of Malmesbury

the use of land components as the basic mapping units which do not align with existing land use boundaries.

Because perennial crops are potentially more profitable per area unit than wheat, it is fair to conclude that the non-urban and non-wetland areas in Figure 9-26 are currently not being optimally used. Based on this preliminary analysis, they may potentially be suitable for perennial crops. With an annual rainfall of $460 \mathrm{~mm}$, this area is significantly drier than the Stellenbosch area and it will therefore have to rely much more on irrigation. Alternatively, dry-land vineyards or olives can be considered. A more detailed (large-scale) survey incorporating factors like local soil properties and irrigation infrastructure could be conducted to identify specific areas suitable for intensive perennial crop cultivation.

\subsection{DISCUSSION}

This chapter has aimed to demonstrate how CLUES can be used to interactively and rapidly conduct suitability analyses in the Western Cape. The system offers the functionality to set and store detailed land use requirements and produce high-quality suitability maps within seconds. A series of suitability maps of varying scales were created for the greater Cape Town, Stellenbosch and Swartland/Malmesbury areas according to ten land requirements for perennial crop production. Although the results can be improved by incorporating more detailed data and by setting better rules and weights, the analyses have succeeded to illustrate the capabilities of the system. The demonstration clearly shows that the strength of CLUES is not only its ability to carry out suitability analyses, but that its true powers lie in its ability to facilitate the userfriendly assimilation of expert knowledge related to land uses and to rapidly generate spatial visualizations of the products of the analyses. The highly automated environment enables experts to interactively see the individual and combined effects on suitability by applying rules and weighting schemes. In addition, the web-based platform of CLUES allows experts to use the 
functionality from any computer with Internet access. This not only improves usability, but also increases accessibility as special software and licenses are not required. However, the use of web technology does limit users to the data stored in the land unit database because users are not allowed to include external data in their analyses. This restriction, along with other specific limitations of CLUES and web technology generally, are discussed in the next chapter. 


\section{CHAPTER 10: EVALUATION OF THE RESEARCH}

Web technology may provide a solution to the high cost of SDSS and GIS as it eliminates the need for expensive hardware or software. Web mapping has shown that the Internet is a costeffective way in which spatial information can be delivered to many users. The spatial analysis functionality employed by SDSS is however difficult to implement using web technology. This is mainly due to the complexities involved in managing the data used, created and updated in such spatial analysis operations. To date, this limitation has impeded the use of the Internet for SDSS applications.

The demonstrations described in the previous chapter confirm that spatial analysis functionality can be implemented using standard (i.e. web browser compatible) web technologies. The abilities and limitations of CLUES specifically and web technology generally are assessed in this chapter and in conclusion the study's aims and objectives are revisited to critically evaluate the research results. Suggestions for future research are finally offered.

\subsection{ASSESSMENT OF CLUES}

To investigate the potential of web technology for SDSS development, a system was needed that incorporates the functionality most frequently associated with SDSS. The most important property that a SDSS should exhibit is the ability to interactively generate different geographical scenarios through automated modelling and directed spatial analysis. This ability is especially important in land suitability analysis as it involves multiple factors of varying importance that are often difficult to define. By interactively visualizing the effects of different land use requirements, the user can gain a better understanding of the dynamics and complexities of land uses and their related properties.

CLUES was designed to encompass the functionality of SDSS with particular application to land evaluation. Given that the aim was to determine the potential of the Internet for SDSS development, the system had to be implemented using existing web technology so that Internet users would be able to carry out land evaluation using a standard web browser.

The success of a software product is usually measured against its ability to meet the requirements identified during the requirement analysis. A requirement analysis expresses the needs and constraints placed on a software product and precedes most large software developments. A software requirement is a property that the software must exhibit to solve a particular problem or perform a certain function. 
Drawing on an extensive literature study, a range of functional (i.e. what the system should do) and operational (i.e. how the system should do it) requirements was identified (Section 4.1). The data needed to support the functional and operational requirements was also specified. The following sections revisit these requirements in order to review the success of CLUES as a webbased land evaluation system. In each case further system design and implementation suggestions are offered, together with ideas for further systematic research in this field.

\subsubsection{Functionality requirements}

The main functional requirement of CLUES is to produce land use suitability maps. Chapter 9 records how CLUES can be used to carry out land suitability analyses and it testifies that most of the related functional requirements were implemented. It demonstrated that one is able to define land uses, set suitability rules, execute evaluations and create suitability maps. The only functional requirement that could not be implemented in CLUES is the ability for users to load and prepare their own spatial data for analysis. This functionality was excluded due the security risk associated with uploading data to web servers. A shared spatial database (i.e. a land unit database) was consequently developed and populated with fundamental data sets for land evaluation.

The process of setting land requirement rules is highly flexible as users are allowed to specify Boolean and fuzzy rules for individual suitability levels. Users can also manipulate the importance of each land property by assigning weights to individual land requirements while a unique set of land requirements can be defined for each land use that needs to be analysed. Careful consideration must be given to the relative weightings of land use requirements as these strongly influence the outcome of a suitability analysis. Decisions about weightings should be supported by techniques such as Saaty's (2003) analytical hierarchical process (AHP) or pairwise comparison (Malczewski 1999).

The mapping capability of CLUES is one of its most powerful functions in that it allows users to spatially visualize different land use scenarios. The mapping environment is intuitive and provides a range of tools through which users can produce suitability maps. Users can also produce reports on individual or selected land units.

Although the main functional requirements of CLUES have been met, much additional functionality should be considered to improve the system. A tool whereby users can produce an optimal land use map would greatly enhance the system's usefulness. Such a map can be generated by assigning to a land unit the land use having the highest suitability rating. The tool should permit users to select the land uses to be considered in the analysis and also allow them to 
specify targets for the areas that each land use should cover. For agricultural land uses these targets might reflect current demand for produce or market prices. By including prices and average yields in the knowledge base, maps of optimal income can be produced. Such maps will be very helpful to agricultural economics planning.

In the current version a satellite image is provided with which users can orientate themselves. It may be convenient to supplement the satellite image with additional layers such as roads and annotations to better orientate a user. However, care must be taken to keep the mapping environment simple and easy to use. Too many layers and additional map information might confuse users, especially those unfamiliar with GIS or similar software. Orientation layers should therefore be deactivated by default, but an option to activate them should be included.

The present version of CLUES does not allow for individual land properties to be viewed spatially. Although it is possible to view the effect of a single land use requirement on a suitability analysis, it may be helpful to allow users to produce maps of individual land properties so that they can familiarize themselves with the data in the land unit database. The inclusion of hundreds of land property layers in the mapping environment will inevitably cause confusion. A possible solution to this problem is to develop a second map viewer in which the individual land properties can be examined.

The system now offers a tool whereby users can import existing land use requirements from the central knowledge base. These land requirements should be extended to include more land uses so that users can work more efficiently. Requirement specifications should be based on expert knowledge and relevant literature, and the sources of the requirements must be properly referenced. CLUES will benefit from functionality that facilitates AHP and related consistency calculations.

The reporting capabilities of CLUES need to be improved. A worthwhile augmented report would be one that provides an overview of a land use evaluation. Such a report might include summaries of the rules and land use requirements used during the suitability analysis as well as statistics about the analysis results. An outline of the relative proportions (i.e. area) of each suitability level can, for instance, be provided. In addition, information about the specific area under consideration can be compared with that of the entire province. This kind of overview will be especially beneficial to environmental managers who are concerned with provincial and national conservation targets.

Although the use of land components as mapping units (land units) is effective for representing natural variables (e.g. effective soil depth, annual rainfall, heat units), they often do not coincide with man-made boundaries (e.g. urban edges and conservation area limits). This may cause 
inconsistent results when man-made features are considered in suitability assessments. A possible solution is to split land units along the boundaries of selected features. However, care must be taken to only incorporate essential boundaries as the splitting process may significantly increase the number of land units, causing increased storage requirements and longer processing times, particularly during suitability analyses.

\subsubsection{Operational performance requirements}

The operational performance of a system qualitatively describes how well it performs its primary function. The following sections evaluate CLUES in terms of its accessibility, speed, userfriendliness, and data storage capabilities.

\subsubsection{System accessibility}

Improved accessibility is one of the main advantages of using web technology for SDSS development. For demonstration purposes CLUES runs on an intranet and is accessible only to computers on the Stellenbosch University local area network (LAN). However, the system can be conveniently migrated to a web server open to all Internet users. This will allow anyone with access to a computer and the Internet to use the system.

Users do not need expensive GIS or expert system licenses to use CLUES. The only software required is a freely available web browser. This means that CLUES is platform independent and can be used on all major operating systems like Microsoft Windows, Linux, UNIX or Apple Macintosh. CLUES requires limited computer resources as most of the processing is carried out on the server. Even a computer with a modest hardware configuration or a mobile device such as a cellphone can be used.

\subsubsection{Operational speed}

Although the centralization of computer processing (i.e. a server-client approach) eliminates the need for sophisticated hardware and software on the client-side (i.e. user), it has implications as to the hardware and processing speed of the server. Because suitability analysis is computationally intensive - each record (i.e. land unit) in the land unit database must be considered and rated in terms of its land properties - an analysis can take a long time to complete.

In the requirement analysis it is specified that the response time of CLUES should be similar to that of a GIS. In addition, a maximum response time of 60 seconds is specified as this is the maximum time that a web user is willing to wait for a web page to load (see Section 4.1.2.2). Due to the size of the land unit database, however, it was determined that a tabular calculation 
that emulates a suitability analysis takes more than three minutes to complete in ArcGIS 9.2. Although the performance of CLUES is similar to that of ArcGIS, a response time of 60 seconds is clearly not a realistic target.

An SDSS is meant to enable users to interact with spatial data to better understand semistructured and complex geographical problems. A major advantage of SDSS over GIS is that it facilitates scenario building. Because response times in excess of three minutes inhibit interaction and will inevitably frustrate users, a measure was implemented that reduces the number of land units considered during a suitability analysis. By limiting the evaluation to those land units visible on the map at the time of initiating an analysis, response times were substantially improved to less than one minute (at a scale of 1:50 000). In addition, because the processing time increases exponentially as the map scale is reduced, analyses at scales smaller than 1:1000 000 were disabled.

Response times can be improved if CLUES is migrated to a more powerful server. The modest server on which the system now runs is not really suitable for intensive processing. Multiple servers may even become necessary as the number of concurrent users increases.

Another factor influencing response time is Internet bandwidth. Although very little bandwidth is required for most of the operations for setting of land use requirements, the mapping component of CLUES is image intensive which may cause delays on slow Internet connections. The size of the CLUES map frame was intentionally made small to keep bandwidth requirements at a minimum.

\subsubsection{System user-friendliness}

Regarding the user-friendliness of the system, all of the requirements set out in Section 4.1.2.3 were implemented in the design and construction of the GUI. The interface was kept as simple as possible whilst including all the necessary functionality. The appearances of menus, forms and tables are consistent on all pages to prevent confusion and users are led through the process with appropriate feedback and help.

To support the setting of rules, graphics are used to visualize the effect of rule thresholds. The visualization of fuzzy functions especially can be improved with the help of other web technologies such as shock wave flash (SWF). Because the graphics representing individual rules are constructed from oblique images, fuzzy rules are partly obscured by one another. With SWF, rules can be made transparent. It will also allow for more complex fuzzy functions (such as the sfunction) to be implemented. 
The mapping and analysis environment is intuitive even for users who are unfamiliar with GIS software. With a little practice, users can create high-quality maps using a range of available controls. These include panning, zooming and enabling/disabling layers for display. Suitability maps are automatically produced from within the mapping environment by simply selecting the appropriate land use from a drop-down list. For better readability, suitability maps are rendered in colours identical to the colours assigned to the suitability levels in the rulebase. Results can therefore be related to specific rule sets, hence supporting the interpretation of results as well as the interactive fine-tuning of rules.

\subsubsection{Data storage mode and capacity}

The use of land units as basic mapping units enabled the storage of suitability analysis results in the land unit database. Because these results are only stored temporarily (until the user ends the session), the storage space requirements remain constant. This means that there is no danger that the system will become unstable due to the creation of overly large volumes of data by users. The database is also protected from corruption by virtue of the storage and retrieval of data being internally managed by the system.

\subsubsection{Data requirements}

Although all the data sets specified in the requirement analysis were acquired, not all of these were available at the specified scale of 1:50 000. The following sections overview the quality of the data in the land unit database and make suggestions on how it can be improved.

\subsubsection{Soil data}

The soil data currently used by the system was derived from land type data, published at a scale of 1:250 000. This data is not detailed enough to work in land suitability analyses at a scale of 1:50 000. Ideally, it should be replaced by more detailed data mapped at scales of 1:50 000 or larger. Unfortunately, the land type data is the only existing soil data covering the entire Western Cape. Although semi-detailed to detailed (i.e. 1:50 000 to 1:1 000) soil surveys have been conducted for selected areas in the Western Cape, only some of this data is available in GIS format. Much time and financial resources will be required to collect and capture this data into a suitable format for use in suitability analyses. Moreover, the major part of the Western Cape has not been surveyed at large scales. This means that land type data will, for the foreseeable future, remain the only soil data source for the larger part of the Western Cape.

The land type data can be further analysed to estimate other variables such as soil $\mathrm{pH}$ and fertility. To do so, each soil series in each land type must be determined and interpreted to 
calculate average values that are representative of all the soil series in a land type. Owing to the large number of soil series in each land type, an algorithm will have to be developed to automate this process.

Because most of the data contained in the land types is based on terrain descriptions, it is possible to spatially enhance the data with the aid of terrain analysis (Van Niekerk \& Schloms 2002). However, more research is needed to determine the accuracy of such enhancements.

A methodology is also needed to use soil data at different scales in CLUES, including the semidetailed soil data available in GIS format. This will significantly improve suitability analysis in areas for which the semi-detailed data is available. To avoid any inconsistencies occurring in the results obtained from different soil data sources, users should be informed about the quality of the data used in an analysis and they should also be advised to interpret the results accordingly.

Techniques should be explored to predict soil distributions using more easily measured environmental variables for areas in which semi-detailed data is not available. These so-called soil-landscape models are increasingly being employed to supplement and even replace conventional soil maps (Park, McSweeney \& Lowery 2001). Soil-landscape modelling is the application of statistical techniques to predict the spatial distribution of soils using terrain and other environmental variables (Hengl, Gruber \& Shrestha 2004). The technique is based on the premise that there is a strong relationship between soil and topography (Jenny 1941). Although more research is needed to determine the accuracy of soil-landscape model predictions (McBratney, Santos \& Minasny 2003; Thwaites \& Slater 2000), the technique shows potential, especially in the Western Cape for which relatively good terrain data is available (Van Niekerk \& Schloms 2002).

\subsubsection{Terrain data}

The WCDEM was used as the terrain data source. According to the accuracy assessment reported in Section 5.1.3, no DEM of the Western Cape has a higher quality than the WCDEM and it was found to be superior in terms of both spatial resolution $(20 \mathrm{~m})$ and vertical accuracy (7m). The WCDEM was however too detailed to be analysed as a unit in CLUES and was consequently rescaled to $80 \mathrm{~m}$ resolution. The vertical accuracy $(9 \mathrm{~m})$ of the resulting WCDEM80 was not significantly effected and was still superior to other DEM of the Western Cape.

The WCDEM80 was used in CLUES as the source for elevation information and to generate the terrain derivatives slope gradient, slope aspect and curvature. It was also used to delineate land components using multi-resolution image segmentation. The land components were used as basic mapping units (land units) for suitability analysis. 


\subsubsection{Climate data}

The climate data included in the land unit database was generated at a resolution of 90 metres by far the most detailed climate data set currently available. Unfortunately, this data set consists of only average monthly temperature and rainfall variables. Further analysis is required to derive other variables useful in suitability analyses, for example chill units, heat units, continentality, a summer aridity index and precipitation seasonality. These climatic indicators can be generated in a GIS and loaded into the land unit database. Alternatively, functionality can be developed in CLUES that will generate the indices from the climate variables already in the database. Land properties calculated on demand can be considered as 'virtual' properties as they are not physically stored in the land unit database. This will not only save storage space but will allow users to manipulate and fine-tune land properties to their specific needs.

\subsubsection{Scale, scalability and flexibility requirements}

Although the current version of CLUES can be used in an operational environment, its application is limited by the number of land property data sets in the land unit database. The number of available land properties should be extended so that users can have more freedom in terms of land requirement construction.

As discussed in Section 10.1.3.1, the scale of the land unit data influences the level at which land evaluations can be done. Users must be made aware that the suitability maps generated by CLUES are not meant to be used to support land use management decisions on a local or farm level. The maps provide an overview of land suitability on municipal and provincial levels and should be used as a preliminary indication of areas that might be suitable for a particular use. Such areas should then be targeted for detailed analyses based on data captured at larger $(>1$ : 10 000) scales.

The principles by which CLUES carries out suitability analyses are not related to scale. Consequently, it is possible to modify the system to perform analyses at more detailed levels if the data is available. Conversely, by simply modifying the land unit database, the system can be configured to be used for another province or even on a national or global level. If such modifications result in a substantial increase in land units, a more robust DBMS may be required.

It is important to note that although the size of the land unit database can be extended, any increase in the number of land units will influence the response times of the system as there is a direct relationship between execution times and number of land units. For implementations where the number of land units exceeds one million, it is recommended that multiple land unit 
databases be used in order to reduce load. An additional menu or map can be included in the GUI that will allow users to choose the area (or land unit database) in which they would like to work. Such a differential approach will improve response times considerably and will effectively support an unlimited number of land units.

Another limitation of the existing land unit database is that it can only support 255 fields (i.e. attributes). Because land properties are stored as attributes of each land unit (i.e. record), each land property requires a field in the database. Consequently, there is a limit to the number of land properties that can be stored in the database. The maximum number of land properties also depends on the number of active users because each user is assigned a field for analysis purposes. It is unlikely that the number of users will exceed 50 at any time, which means that there are 200 (less five for other operational purposes) fields available for the storage of land properties. If storage for more than 200 land properties is needed, the database can be replaced by an enterprise DBMS such as Oracle, which can accommodate up to 1000 fields. Owing to factors such as the WMS and web server loads, a better solution would be to set up separate servers, each with a different instance of CLUES. As with most enterprise web applications, a central user management database can be created to direct users to the appropriate instance of CLUES.

During suitability analysis, the attributes (i.e. land properties) of each land unit in the land unit database are considered and evaluated against the rules in the knowledge base. By doing so CLUES essentially emulates one of the most fundamental spatial analysis operations, namely overlaying. Although logically delineated units representing terrain morphology (i.e. land components) are used as the basic mapping units in CLUES, other units may also be employed. Regular tessellations of square regions can for instance be used to emulate most raster GIS overlying operations. This approach is highly effective as it eliminates the need for complex spatial actions usually associated with vector overlaying. Operations such as finding intersections between overlaying futures, splitting features, and creating new records and fields are very processing-intensive, especially for large and complex areas such as the Western Cape.

CLUES was developed to investigate the potential of web technology for SDSS development in a sense it is a case study of a web-based SDSS for land evaluation. Its development has provided a good understanding of the available technologies and given insight into the capabilities and limitations of web technology for SDSS development. A synopsis of the potential of web technology for SDSS development is presented in the next section. 


\subsection{POTENTIAL OF WEB TECHNOLOGY FOR SDSS DEVELOPMENT}

Web technology holds much potential for SDSS development as it provides a cost-effective and intuitive way in which directed spatial analysis functionality can be delivered to a wide audience. Web-based SDSS such as CLUES are attractive to users because they are highly accessible and free. Users are also likely to find web-based SDSS intuitive and easy to use as the user interface consists of web pages and other web components that are familiar.

Web-based SDSS offer significant benefits to developers because much of the costs related to the distribution and maintenance of software are eliminated with client-server web technology. In contrast to local (i.e. desktop) SDSS, updates can be made on a continuous basis without seriously inconveniencing users. In effect, there is only one always up-to-date version of the system at any given time. This simplifies support, maintenance and training activities.

The centralized way in which web-based systems store and distribute data is appealing to users and developers. Many users do not have access to the spatial data required by many desktop SDSS. The provision of preconfigured data sets enables users to obtain results within minutes of entering the system as no data collection is required. In addition, it eliminates the need for users to carry out data manipulations such as coordinate system, map projection and datum conversions which, when done incorrectly, can have serious consequences regarding incorrect analysis results. The disadvantage of not allowing users to upload their own data is that they are bound by the available data, restricting them to the available land properties, and also limiting their analyses to a particular region.

A significant advantage of web-based SDSS is that reformatting of data to comply with the system requirements prior to development, reduces system development time by avoiding functionality to handle different formats and data types. Less time can also be spent on implementing robust data management and security measures to prevent data corruption.

Security breaches are a major risk in web applications. In spite of the continuous efforts by software developers and governments to reduce Internet-related crimes, many web users have become victims of Internet fraud or have inadvertently downloaded malicious software. By disabling the uploading of data to the web-based SDSS, much of the security risk is eliminated. Administrators must nevertheless ensure that server backups are made regularly to prevent data loss.

One of the main limitations of web technology for SDSS deployment is response times as some Internet connections are slow and can cause delays, especially when large maps (i.e. images) are frequently downloaded. However, due to the large databases involved in SDSS, the major cause 
of delay is more likely to be the processing of the tabular and spatial information. This is true for CLUES which is unable to carry out a suitability analysis for the entire Western Cape within an acceptable waiting period (i.e. 60 seconds). This limitation is directly related to the size of the spatial database as similar delays occur when the same operations are carried out using a desktop GIS. The delay is a result of the insufficient data-processing capability of the DBMS and is not attributable to web technology per se. In general, web applications are less responsive than their desktop counterparts as most actions are carried out by the web server. While the processing time on the web server is comparable with that of a desktop application, additional delays are created when a request for data is sent to the web server and when the resulting information is transmitted back as web pages. The combined effect of these delays can cause a latency that can frustrate users with slow Internet connections. However, on faster connections delays are almost unnoticeable, especially to frequent Internet users who have become accustomed to short delays.

Another limitation of web-based SDSS is the restrictions imposed by web technology concerning user-interface sophistication. Unlike desktop applications that can use almost unlimited graphics, web applications are limited by the standard markup and scripting languages used to develop them. Although most of the graphical requirements of CLUES are met by the use of simple image constructs and HTML, more advanced graphic capabilities may be required by other webbased SDSS. For instance, an application might require input mechanisms that allow users to change weightings by using animated sliders. Such advanced interface functionality is not available using HTML and will have to be implemented using SVG or SWF. Unfortunately, these technologies are not native to all web browsers and might not work on all systems.

In spite of the graphical restrictions of web technology, most of the mapping functionality needed by SDSS can be implemented using existing WMS. Although ArcIMS was used to develop CLUES, it is only one of the many WMS available. In addition to a number of other proprietary solutions, there are several open-source WMS that can be freely downloaded from the Internet. These products are frequently updated with new functionality and should be considered for future web-based SDSS implementations. Because the source code of opensource products is available, the potential for customization is virtually unlimited. However, proprietary products such as ArcIMS are considered to be more user-friendly and better documented, hence requiring less development time.

WMS are aimed at producing web maps and are not equipped with spatial analysis functionality. Most of the spatial analysis operations required by SDSS can nevertheless be developed using existing web technology. Not only can overlaying be emulated using fixed mapping units and standard DBMS functions, but operations such as proximity and connectivity can also be carried 
out using scripting. In addition, many enterprise DBMS, such as Oracle and Informix, have introduced spatial extensions to their products that offer a range of GIS-like operations. However, more research is required to investigate the potential of these extensions for the development of web-based SDSS.

The development of CLUES has shown that web technology offers many opportunities for the deployment of spatial analysis and modelling functionality. The generation process exposed many advantages and limitations of the currently available technology as discussed in this section. In the final section the research aims and objectives are revisited in order to evaluate the successfulness of this study.

\subsection{RESEARCH OBJECTIVES REVISITED}

The main aim of this research was to investigate the potential of web technology as a platform for delivering SDSS functionality to a wide audience. As an experiment, a web-based land use expert system was developed called the Cape Land Use Evaluation System (CLUES). The motive for developing the system was to gain insights into the abilities and limitations of available web technologies.

The first objective (see Section 1.6) was to review the literature to determine what functionality is needed by a land evaluation system. The literature on each step in the land evaluation procedure and the three approaches to land suitability analysis, namely Boolean overlay, multicriteria decision making (MCDM) and expert systems, was methodically reviewed (Chapter 2). The literature survey also revealed helpful information about web technologies available for the development of a web-based SDSS and special attention was given to the technologies related to web mapping applications (Chapter 3).

The study of current technologies and existing SDSS was not only instrumental in doing the requirements analysis and designing CLUES (Chapter 4), but also helped identify what data was needed to demonstrate the functionality of the system. This requirement led to the second research objective, namely to collect and prepare fundamental data sets to test and demonstrate CLUES (Chapter 5). Although good-quality climate and terrain data sets were obtained, a general lack of detailed soil data necessitated the use of smaller scale (i.e. 1:250 000) landtype data.

The third and most comprehensive objective of the research was to design, develop and implement the web-based land evaluation system. Land evaluation was chosen as the SDSS application as it strongly relies on spatial analysis - the cornerstone of SDSS. An expert system approach was taken to develop CLUES which consists of a land unit database, a knowledge base 
and a website. The activities related to the development of each of these components are described in Chapters 6, 7 and 8 respectively. Most of the requirements set out in Chapter 4 were successfully implemented in the resulting system.

The demonstration of CLUES (Chapter 9) constituted the fourth research objective. The functionality of the system was illustrated by carrying out land suitability analyses of perennial crops in the greater Cape Town, Stellenbosch and Swartland regions. The resulting land use scenarios are realistic and informative. They did, however, expose some limitations of the system and the available data. This is reported in Chapter 10, which addresses the final objective of the research, i.e. to critically evaluate and make recommendations about CLUES, and draw attention to the general limitations and potentials of web technology for web-based SDSS development. The evaluation showed that available web technology offers excellent opportunities for the deployment of spatial analysis and modelling functionality to a wide audience.

\subsection{CONCLUSION}

Products like Google Earth have spotlighted the value of web mapping technology for spatial decision support and they have demonstrated the potential of such tools for the cost-effective distribution of maps and other spatial information to improve productivity and to aid decision making. But the functionality of most web mapping applications is limited to data display and does not support more advanced functionality, such as spatial analysis and modelling, needed for SDSS development. This research has shown that web mapping technology can be extended to include this functionality by combining standard web mapping technology and database management systems with client-side and server-side web programming. The techniques developed here can be used to implement and distribute powerful spatial analysis functionality to Internet users. To date, such functionality has been the domain of those with access to expensive GIS software and the necessary data. A web-based SDSS such as CLUES has the potential to dramatically increase access to spatial analysis functionality since anyone with access to a modest computer and the Internet can use the systems.

Increased usage of web-based SDSS is likely to help improve spatial awareness because users will gain a better understanding of the possibilities of spatial technologies. Increased accessibility is likely to stimulate an increase in demand for additional functionality which in turn will inspire the development of better technology. One can anticipate that web-based SDSS will boost the current upward trend in the online use of maps and geographical tools, and also that new SDSS will be developed for various applications. Such online SDSS are expected to become valuable sources of spatial information and they will also provide mechanisms through 
which users can store, analyse and share expert knowledge to make better spatial decisions. Web technology is a medium possessing the unique ability to act as an intermediary between collaborating individuals to find solutions to complex geographical problems facing our increasingly complex world. It is expected that the capacity of web technology to cost-effectively deploy geographical information and functionality to a wide audience will bring the so-called 'unfinished GIS revolution' to an end and that web-based SDSS will help support earth-changing decision making. 


\section{REFERENCES}

Abran A, Moore JW, Bourque P \& Dupuis R (eds) 2004. The software engineering body of knowledge guide. Los Alamitos: IEEE Computer Society Professional Practices Committee.

Adediran AO, Parcharidisb I, Poscolieric M \& Pavlopoulosd K 2004. Computer-assisted discrimination of morphological units on north-central Crete (Greece) by applying multivariate statistics to local relief gradients. Geomorphology 58: 357-370.

Agrell PJ, Stam A \& Fischer GW 2004. Interactive multiobjective agro-ecological land use planning: The Bungoma region in Kenya. European Journal of Operational Research 158: 194-217.

Al-Najjar B \& Alsyouf I 2003. Selecting the most efficient maintenance approach using fuzzy multiple criteria decision making. International Journal of Economics 84: 85-100.

Altech Netstar 2008. Netstar [online]. Available from http://www.netstar.co.za/content/home/home.aspx [Accessed 1 July 2008].

Antonio G \& Signorini A 2005. The indexable Web is more than 11.5 billion pages. Paper delivered at the WWW conference, Chiba.

Argialas DP 1995. Towards structured-knowledge models for landform representation. Zeitschrift für Geomorfologie N.F 101: 85-108.

Ascough JCI, Rector HD, Hoag DL, McMaster GS, Vandenberg BC, Shaffer MJ, Weltz MA \& Ahjua LR 2001. Multicriteria spatial decision support systems for agriculture: Overview, applications, and future research directions. In Rizzoli AE \& Jakeman AJ (eds) Integrated assessment and decision support proceedings of the 1st Biennial Meeting of the IEMSS. June 24-27 2002, 175-180. Lugano: IEMSS.

Bailey B s.d. User interface design update [online]. Insights from Human Factors International Inc. Available from http://www.keller.com/articles/downloadspeed.txt [Accessed 6 February 2008].

Basson FC 2005. A spatial decision support system for groundwater abstraction impact assessment and licensing. MSc thesis. Stellenbosch: Stellenbosch University.

Berners-Lee T 1989. Information management: A proposal [online]. Geneva: CERN. Available from http://www.w3.org/History/1989/proposal.html [Accessed 18 Oct 2005]. 
Berners-Lee T 1991. Re: status. Re: X11 BROWSER for WWW [online]. Available from http://lists.w3.org/Archives/Public/www-talk/1991SepOct/0003.html [Accessed 31 October 2007].

Bester FJ 2004. Multi-criteria decision making and geographical information systems: An extension for ArcView. MSc thesis. Stellenbosch: Stellenbosch University.

Bolstad PV \& Smith JL Errors in GIS: Assessing spatial data accuracy. In Lyon JG \& McCarthy $\mathrm{J}$ (eds) Wetland and environmental applications in GIS, 301-312. New York: Lewis.

Bosshard A 2000. A methodology and terminology of sustainability assessment and its perspectives for rural planning. Agriculture, Ecosystems \& Environment 77: 29-41.

Botes A, McGeoch MA, Robertson HG, Van Niekerk A, Davids HP \& Chown SL 2006. Ants, altitude and change in the northern Cape Floristic Region. Journal of Biogeography 33: 7190.

Brown RB 2003. Soil texture [online]. Gainesville: University of Florida. Available from http://edis.ifas.ufl.edu/SS169 [Accessed 11 September 2007].

Bruno R, Follador M, Paegelow M, Renno F \& Villa N 2006. Integrating Remote Sensing, GIS and Prediction Models to Monitor the Deforestation and Erosion in Peten Reserve, Guatemala. Society for Mathematical Geology XIth International Congress, Université de Liège, Belgium.

Buckle C 1996. Weather and climate in Africa. Harlow: Longman.

Burrough PA 1989. Fuzzy mathematical methods for soil survey and land evaluation. Journal of Soil Science 40: 477-492.

Burrough PA, MacMillan RA \& Van Deursen W 1992. Fuzzy classification methods for determining land suitability from soil profile observations and topography. Journal of Soil Science 43: 193-210.

Burrough PA \& McDonnel RA 1998. Principles of geographical information systems. Oxford: Oxford University Press.

Campbell JB 2006. Introduction to remote sensing. London: Taylor \& Francis.

Canadian Conservation Institute 2005. Analytical Hierarchy Process (AHP) program [online]. Ottawa: Canadian Conservation Institute. Available from http://www.cciicc.gc.ca/tools/ahp/index e.asp [Accessed 10 July 2008]. 
Canter S 2004. Understanding Client-Side Scripting [online]. PC Magazine. Available from http://www.pcmag.com/article2/0,1759,1564972,00.asp [Accessed 1 June 2008].

CapeNature 2007. CapeNature reserves [online]. Cape Town: WCNCB. Available from http://www.capenature.com/index.php?fSectionId=3 [Accessed 31 August 2007].

Caquard S 2003. Internet, maps and public participation: Contemporary limits and possibilities. In Peterson MP (ed) Maps and the Internet, 345-357. Amsterdam: Elsevier.

Carey VA 2005. The use of viticultural terroir units for demarcation of geographical indicators for wine production in Stellenbosch and surrounds. $\mathrm{PhD}$ dissertation. Stellenbosch: Stellenbosch University, Dept of Viticulture and Oenology.

Cartwright W 2003. Maps on the Web. In Peterson MP (ed) Maps and the Internet, 35-56. Amsterdam: Elsevier.

CDSM 2007a. Digital elevation model (DEM) [online]. Cape Town: CDSM. Available from http://w3sli.wcape.gov.za/ [Accessed 21 September 2007].

CDSM 2007b. Maps of the national series [online]. Cape Town: CDSM. Available from http://w3sli.wcape.gov.za/ [Accessed 21 September 2007].

Ceballos-Silva A \& López-Blanco J 2003a. Delineation of suitable areas for crops using a multicriteria evaluation approach and land use/cover mapping: A case study in Central Mexico. Agricultural Systems 77: 117-136.

Ceballos-Silva A \& López-Blanco J 2003b. Evaluating biophysical variables to identify suitable areas for oats in Central Mexico: A multi-criteria and GIS approach. Agriculture, Ecosystems and Environment 95: 371-377.

Chang K 2006. Introduction to geographic information systems. 3rd ed. New York: McGraw Hill.

Clarke KC 2003. Getting started with geographic information systems. Upper Saddle River: Prentice Hall.

Clarke M 1990. Geographical information systems and model-based analysis: Towards effective decision support systems. In Scholten HJ \& Stillwell JCH (eds) Geographical information systems for urban and regional planning, 165-175. Amsterdam: Kluwer Academic Publishers.

CNDV Africa 2005. Western Cape provincial spatial development framework [online]. Cape Town: PGWC. Available from http://www.capegateway.gov.za/eng/pubs/guides/W/120505 [Accessed 11 March 2008]. 
Codd EF 1970. A relational model of data for large shared data banks. Cummiciations of the ACM 13: 377-387.

Cools N, De Pauw E \& Deckers J 2002. Towards an integration of conventional land evaluation methods and farmers' soil suitability assessment: A case study in northwestern Syria. Agriculture, Ecosystems and Environment 1968: 1-16.

Dai FC, Lee CF \& Zhang XH 2001. GIS-based geo-environmental evaluation for urban land-use planning: A case study. Engineering Geology 61: 257-271.

Davidson DA (ed) 1986. Land evaluation. New York: Van Nostrand Reinhold.

Davidson DA, Theocharopoulos SP \& Bloksma RJ 1994. A land evaluation project in Greece using GIS and based on Boolean and fuzzy set methodologies. International Journal of Geographical Information Systems 8: 369-384.

De Kok R, Schneider T \& Ammer U 1999. Object-based classification and applications in the alpine forest environment. International Archives of Photogrammetry and Remote Sensing 32: part 7-4-3.

De la Beaujardiere J 2004. OGC Web map service interface [online]. Available from http://www.opengeospatial.org/standards/wms [Accessed 7 February 2008].

De la Rosa D 2002. MicroLEIS DSS: A land evaluation decision support system for agricultural soil protection [online]. Seville: Instituto de Recursos Naturales y Agrobiología de Sevilla. Available from http://irnas106.irnase.csic.es/microlei/manual2/overview.htm\#top [Accessed 13 October 2006].

De la Rosa D, Mayol F, Diaz-Pereira F, Fernandez M \& De la Rosa D 2004. A land evaluation decision support system (MicroLEIS DSS) for agricultural soil protection with special reference to the Mediterranean region. Environmental Modelling \& Software 19: 929-942.

Definiens Imaging 2004. eCognition 4 User Manual. Munich: Definiens Imaging GmbH.

Definiens Imaging 2007. Definiens Developer (version 7) user manual. Munich: Definiens AG.

DeMers MN 2005. Fundamentals of geographic information systems. 3rd ed. New York: Wiley \& Sons.

Dendgiz O, Bayramin Ü \& Yüksel M 2003. Geographic information system and remote sensing based land evaluation of BeypazarÝ area soils by ILSEN model. Turkish Journal of Agriculture and Forestry 27: 145-153. 
Dennis AR \& Taylor NJ 2006. Information foraging on the web: The effects of "acceptable" Internet delays on multi-page information search behaviour. Decision Support Systems 42: 810-820.

Densham PJ 1991. Spatial decision support systems. In Maguire DJ, Goodchild MF \& Rhind DW (eds) Geographical information systems: Principles and applications, 403-412. Harlow: Longman.

Dent D \& Young A 1981. Soil survey and land evaluation. London: George Allen \& Unwin.

Dini J 2007. WfWet's year in review: March 2006 to April 2007 [online]. Cape Town: SANBI. Available from http://wetlands.sanbi.org/resource.php?id=113 [Accessed 10 March 2008].

Dobbs D 2004. Vital statistics: Web pages that suck [online]. Electronic review of computer books. Available from http://www.ercb.com/feature/feature.0027.html [Accessed 13 May 2008].

Du Toit DA, Mouton PLN, Flemming AF, Van Niekerk A, Day JA \& Schulz R 2002. Climate and the presence of generation glands in female girdled lizards: A case study of the cordylusniger-oelofseni complex. Journal of Herpetology 39: 384-388.

Du Toit DA, Mouton PLN \& Van Niekerk A 2006. Climatic correlates of melanistic cordylid lizards. Paper presented at the 8th Herpetological Association of Africa Symposium, NorthWest University, Potchefstroom.

Dymond JR, Derose RC \& Harmsworth GR 1995. Automated mapping of land components from digital elevation data. Earth Surface Processes and Landforms 20: 131-137.

Eastman JR 2000. Decision strategies in GIS. Directions Magazine Dec 2000: s.p.

Eastman JR 2006. IDRISI Andes: Guide to GIS and image processing. Worcester: Clark University.

Eastman JR, Jin W, Kyem PAK \& Toledano J 1995. Raster procedures for multi-criteria/multiobjective decisions. Photogrammetric Engineering \& Remote Sensing 61: 539-547.

Encyclopaedia Britannica 2007. Artificial intelligence [online]. Encyclopædia Britannica Online. Available from http://www.britannica.com/eb/article-219098 [Accessed 23 Dec 2007].

ESRI 2002a. ArcView GIS online user guide. Redlands: ESRI.

ESRI 2002b. ArcView online help. Redlands: ESRI.

ESRI 2002c. MapObjects. Redlands: ESRI. 
ESRI 2003. ArcIMS scalability supports heavy demand for site. ArcUser January-March: s.p.

ESRI 2007a. ArcGIS 9.2 online user manual. Redlands: ESRI.

ESRI 2007b. ArcIMS 9.2 Installation manual. Redlands: ESRI.

ESRI 2007c. ArcSDE [online]. Available from http://www.esri.com/software/arcgis/arcsde/index.html [Accessed 23 November 2007].

FAO 1976. A framework for land evaluation. Rome: FAO.

FAO 1984. Land evaluation for development. Rome: FAO.

FAO 1985. Guidelines: Land evaluation for irrigated agriculture. Rome: FAO.

Fernandez Ruiz R 2003. Alternative land uses to forestry in the Western Cape: a case study of La Motte plantation. MSc thesis. Stellenbosch: Stellenbosch University.

Fischer G, Granat J \& Makowski M 1998. AEZWIN: An interactive multiple-criteria analysis tool for land resources appraisal. Laxenburg: International Institute for Applied Systems Analysis.

Fleming CC \& Von Halle B 1989. Handbook of relational database design. New York: Addison-Wesley.

Fourie JC 2006. Evaluating agricultural potential of a Cape Metropolitain catchment: A fuzzy logic approach. MSc thesis. Stellenbosch: Stellenbosch University.

Gartner G 2005. TeleCartography: A new means of GeoCommunication. In Taylor DRF (ed) Cybercartography: Theory and practice, 373-387. Amsterdam: Elsevier.

Gibson PJ \& Power CH 2000. Introduction to remote sensing: Digital image processing and applications. London: Taylor and Francis.

Giles PT \& Franklin SE 1998. An automated approach to the classification of the slope units using digital data. Geomorphology 21: 251-264.

GISjobs.com 2006. User survey [online]. Appleton: GISjobs.com, LLC. Available from http://www.gisjobs.com/survey/responses.jsp?countryLoc=all\&sal=N [Accessed 10 February 2006].

Goldstuck A 2004. The next big boom [online]. Pinegowrie: World Wide Worx. Available from http://www.ghostdigest.co.za/code/A 391.html [Accessed 21 Aug 2006]. 
Goodchild MF \& Densham PJ 1990. Research initiative six, spatial decision support systems: Scientific report for the specialist meeting, Technical Report 90-5. Santa Barbara: National Center for Geographic Information and Analysis.

Google 2005. Google launches free 3D mapping and search product [online]. Mountain View: Google Inc. Available from http://www.google.com/press/pressrel/google earth.html [Accessed 18 Oct 2005].

Graff LH \& Usery EL 1993. Automated classification of generic terrain features in digital elevation models. Photogrammetric Engineering \& Remote Sensing 59: 1409-1417.

GRASS 2006. Geographic resources analysis support system [online]. Trento. Available from http://grass.itc.it/ [Accessed 16 February 2006].

Green D \& Bossomaier T 2001. Online GIS and spatial metadata. London: Taylor \& Francis.

Hall DJ \& Khanna DK 1977. The ISODATA method computation for the relative perception of similarities and differences in complex and real data. In Enslein K, Ralston A \& Wilf HS (eds) Statistical methods for digital computers, 340-373. New York: John Wiley \& Sons.

Hall GB, Wang F \& Subaryono 1992. Comparison of Boolean and fuzzy classification methods in land suitability analysis by using geographical information systems. Environment and Planning 24: 497-516.

Hengl T, Gruber S \& Shrestha DP 2004. Reduction of errors in digital terrain parameters used in soil-landscape modelling. International Journal of Applied Earth Observation and Geoinformation 5: 97-112.

Hijmans RJ, Cameron SE, Parra JL, Jones PG \& Jarvis A 2005. Very high resolution interpolated climate surfaces for global land areas. International Journal of Climatology 25: 1965-1978.

Hoersch B, Braun G \& Schmidt U 2002. Relation between landform and vegetation in alpine regions of Wallis, Switzerland. A multiscale remote sensing and GIS approach. Computers, Environment and Urban Systems 26: 113-139.

Houghton JT, Ding Y, Griggs DJ, Noguer M, Van der Linden PJ, Da X, Maskell K \& Johnson CA (eds) 2001. Climate change 2001: The scientific basis. Cambridge: Cambridge Press.

Huajun T, Debaveye J, Da R \& Van Ranst E 1991. Land suitability classification based on fuzzy set theory. Pedologie 61: 277-290.

Huajun T \& Van Ranst E 1992. Testing of fuzzy set theory in land suitability assessment for rainfed grain maize production. Pedologie 42: 129-147. 
IBM 2007. Informix spatial datablade module [online]. Available from http://www306.ibm.com/software/data/informix/blades/spatial/ [Accessed 23 November 2007].

Igué AM, Gaiser T \& Stahr K 2004. A soil and terrain digital database (SOTER) for improved land use planning in Central Benin. European Journal of Agronomy 21: 41-52.

Irvin BJ, Ventura SJ \& Slater BK 1997. Fuzzy and isodata classification of landform elements from digital terrain data in Pleasant Valley, Wisconsin. Geoderma 77: 137-154.

Jackson DI 1999. Climate and fruit plants. In Jackson DI \& Looney NE (eds) Temparate and subtropical fruit production, 7-14. New York: CABI.

James AJ 2001. Die identifisering van ontwikkelingsensitiewe areas teen berghellings:

Stellenbosch- en Hottentotshollandberge. MA thesis. Stellenbosch: Stellenbosch University.

Jankowiski P \& Nyerges T 2001. GIS-supported collaborative decision making: Results of an experiment. Annals of the Association of American Geographers 91: 48-70.

Jarupathirun S \& Zahedi FM 2007. Exploring the influence of perceptual factors in the success of web-based spatial DSS. Decision Support Systems 43: 933-951.

Jenks GF 1967. The data model concept in statistical mapping. International Yearbook of Cartography 7: 186-190.

Jenny H 1941. Factors of soil formation: A system of quantitative pedology. New York: McGraw-Hill.

Jiang B 2003. Beyond serving maps: Serving GIS functionality over the Internet. In Peterson MP (ed) Maps and the Internet, 147-157. Oxford: Elsevier.

Jiang H \& Eastman JR 2000. Application of fuzzy measures in multi-criteria evaluation in GIS. International Journal of Geographical Information Science 14: 173-184.

Joerin F, Theriault M \& Musy A 2001. Using GIS and outranking multi-criteria analysis for land-use suitability assessment. International Journal of Geographical Information Science 15: 153-174.

Joubert SJ 2007. High-resolution climatic variable generation for the Western Cape. MSc thesis. Stellenbosch: Stellenbosch University.

Joubert SJ \& Van Niekerk A 2005. Enhancement of climate data in the South Western Cape using digital elevation models. Paper presented at the Annual South African Geography Students Conference, Cape Town, University of the Western Cape. 
Kalogirou S 2002. Expert systems and GIS: An application of land suitability evaluation. Computers, Environment and Urban Systems 26: 89-112.

Köbben B 2001. Publishing maps on the Web. In Kraak M-J (ed) Web cartography developments and prospects, 73-86. London: Taylor \& Francis.

Kok P \& Collinson M 2006. Migration and urbanization in South Africa. Report 03-04-02. Pretoria: Statistics South Africa.

Kotonya G \& Sommerville I 1998. Requirements engineering: Processes and techniques. West Sussex: John Wiley \& Sons.

Kraak M-J 2001. Settings and needs for web cartography. In Kraak M-J \& Brown A (eds) Web cartography developments and prospects, 1-7. London: Taylor \& Francis.

Lambrechts JJN \& Ellis F s.d. Soil surveys, soil maps, survey methods and land and soil capability evaluation. Stellenbosch: Stellenbosch University.

Land Type Survey Staff 1984. Land types of the maps 2626 Wes-Rand \& 2726 Kroonstad. Pretoria: Department of Agriculture.

Lantican CA, Grierson IT, Chittleborough DT \& Lewis MM 1998. Application of the automated land evaluation system (ALES) and remote sensing for land use planning in the Philippines. Paper presented at the Ninth Australasian Remote Sensing Conference, Sydney.

Lime S 2006. MapServer [online]. Minnesota: University of Minnesota. Available from http://mapserver.gis.umn.edu/ [Accessed 17 November 2007].

Longley PA, Goodchild MF, Maquire DJ \& Rhind DW 2002. Geographic information systems and science. Chichester: John Wiley \& Sons.

Lutgens FK \& Tarbuck EJ 1998. The atmosphere. New Jersey: Prentice Hall.

Lütz M \& Bastian O 2002. Implementation of landscape planning and nature conservation in the agricultural landscape: a case study from Saxony. Agriculture, Ecosystems and Environment 92: 159-170.

Lynch SD 1999. Converting point estimates of daily rainfall onto a rectangular grid. Paper presented at the ESRI User Conference '98, San Diego.

MacMillan RA, Jones RK \& McNabb DH 2004. Defining a hierarchy of spatial entities for environmental analysis and modeling using digital elevation models (DEMs). Computers, Environment and Urban Systems 28: 175-200. 
Mahini AS \& Gholamalifard M 2006. Siting MSW landfills with a weighted linear combination methodology in a GIS environment. International Journal of Environment, Science and Technology 3: 435-445.

Malczewski J 1999. GIS and multi-criteria decision analysis. New York: John Wiley \& Sons.

Malczewski J 2004. GIS-based land-use suitability analysis: A critical overview. Progress in Planning 62: 3-65.

Malczewski J 2006. Ordered weighted averaging with fuzzy quantifiers: GIS-based multicriteria evaluation for land-use suitability analysis. International Journal of Applied Earth Observation and Geoinformation 8: 270-277.

Mantel S, Van Engelen VWP, Molfino JH \& Resink JW 2000. Exploring biophysical potential and sustainability of wheat cultivation in Uruguay at the national level. Soil Use and Management 16: 270-278.

Mantel S, Zhang X \& Zhang G 2003. Identification of potential for banana in Hainan island, China. Pedosphere 13: 147-155.

MapQuest 2008. Mapquest [online]. Denver. Available from http://www.mapquest.com/ [Accessed 1 June 2008].

Marinoni O 2004. Implementation of the analytical hierarchy process with VBA in ArcGIS. Computers \& Geosciences 30: 637-646.

Mau-Crimmins T, De Steiguer JE \& Dennis D 2005. AHP as a means for improving public participation: A pre-post experiment with university students. Forest Policy and Economics 7: 501-514.

McBratney AB, Santos MLM \& Minasny B 2003. On digital soil mapping. Geoderma 117: 3-52.

McDonald RC, Isbell RF, Speight JG, Walker J \& Hopkins MS 1984. Australian soil and land survey. Melbourne: Inkata Press.

McHarg I 1969. Design with nature. New York: Doubleday \& Company.

McSweeney K, Gessler PE, Slater B, Hammer RD \& Bell J 1994. Towards a new framework for modeling the soil-landscape continuum. Soil Science Society of America 33: 127-145.

Microsoft 2007. Microsoft Office Access 2007 top 10 benefits [online]. Available from http://office.microsoft.com/en-us/access/HA101650211033.aspx [Accessed 5 November 2007]. 
Miliaresis GC 2001. Geomorphometric mapping of Zagros Ranges at regional scale. Computers \& Geosciences 27: 775-786.

Mitchell CW 1991. Terrain evaluation. New York: Longman.

Mitchell CW, Webster R, Beckett PHT \& Clifford B 1979. An analysis of terrain classification for long-range prediction of conditions in deserts. Geographical Journal: 72-85.

Mlisa A 2007. Spatial decision support system for hydrological studies in the Table Mountain Group aquifers. MSc thesis. Stellenbosch: Stellenbosch University.

Modine A 2007. Dell makes headway in server market [online]. The Register. Available from http://www.theregister.co.uk/2007/08/23/idc_servers_q207/ [Accessed 2 November 2007].

Mongkolsawat C, Thirangoon P \& Kuptawutinan P 1997. A physical evaluation of land suitability for rice: A methodological study using GIS. Paper presented at the 18th Asian Conference on Remote Sensing, Malaysia.

Mozilla Foundation 2008. About JavaScript [online]. Available from http://developer.mozilla.org/en/docs/About JavaScript [Accessed 1 July 2008].

Mucina L \& Rutherford MC (eds) 2006. The vegetation of South Africa, Lesotho and Swaziland. Pretoria: SANBI.

MWEB 2005. StreetMap [online]. Johannesburg: MWEB. Available from http://new.mweb.co.za/general/streetmap.jsp [Accessed 18 Oct 2005].

Mweso E 2003. Evaluating the importance of soil moisture availability (as a land quality) on selected rainfed crops in Serowe area, Botswana. MSc thesis. Enschede: International Institute for Geo-Information Science and Earth Observation.

Nah F 2004. A study on tolerable waiting time: How long are Web users willing to wait? [online]. Lincoln: University of Nebraska-Lincoln. Available from http://sigs.aisnet.org/sighci/bit04/BIT_Nah.pdf [Accessed 31 August 2007].

NASA 2005. Shuttle Radar Topography Mission [online]. Available from http://www2.jpl.nasa.gov/srtm/mission.htm [Accessed 13 May 2008].

National Geographic Society 2005. Map machine [online]. Washington D.C.: National Geographic Society. Available from http://plasma.nationalgeographic.com/mapmachine/ [Accessed 18 Oct 2005].

Netcraft 2007. August 2007 web server survey [online]. Available from http://news.netcraft.com/archives/web_server_survey.html [Accessed 22 August 2007]. 
netz-tipp.de 2002. Das Internet spricht Englisch - und neuerdings auch Deutsch [online]. netztipp.de. Available from http://www.netz-tipp.de/languages.html [Accessed 19 Oct 2005].

New South Wales National Parks and Wildlife Service 2001. C-Plan: User manaul. Armidale: New South Wales National Parks and Wildlife Service.

Nexen 2007. PHP stats evolution for August 2007 [online]. Available from http://www.nexen.net/chiffres_cles/phpversion/17507php_stats evolution for august_2007.php [Accessed 2 November 2007].

Nielsen J 1994. Ten usability heuristics [online]. Available from http://www.useit.com/papers/heuristic/heuristic list.html [Accessed 4 September 2007].

Nisar Ahamed TR, Rao GK \& Murthy JSR 2000. GIS-based fuzzy membership model for cropland suitability analysis. Agricultural Systems 63: 75-95.

Open Geospatial Consortium 2007. OpenGIS web map service (WMS) implementation specification [online]. Available from http://www.opengeospatial.org/standards [Accessed 24 December 2007].

Open Source Initiative 2007. Home [online]. Available from http://www.opensource.org/ [Accessed 13 May 2008].

Oracle 2007. Oracle spatial \& Oracle locator: Location features for Oracle database 11g [online]. Available from http://www.oracle.com/technology/products/spatial/index.html [Accessed 22 November 2007].

Orlandi E, Ruga L, Romano B \& Fornaciari M 2005. Olive flowering as an indicator of local climate changes. Theoretical Applied Climatology 81: 169-176.

Ortega-Farias SO \& Leon L 2002. Models for predicting apple diameter by using growingdegree days, cultivar Royal Gala. Acta Horticulturae 584: 163-176.

Oxford English Dictionary 2008. Compact Oxford English Dictionary [online]. Oxford: Oxford University Press. Available from http://www.askoxford.com/concise oed/website?view=uk [Accessed 31 January 2008].

Park SJ, McSweeney K \& Lowery B 2001. Identification of the spatial distribution of soils using a process-based terrain characterization. Geoderma 103: 249-272.

Patterson G 2005. Land type survey of South Africa completed [online]. ARC. Available from http://www.arc-iscw.agric.za/main/topnews/landsurvey.htm [Accessed 23-06-2005]. 
Peterson M 2003. Maps and the Internet: An introduction. In Patterson M (ed) Maps and the Internet, 1-16. Oxford: Elsevier.

Pettey C 2007. Gartner says worldwide relational database market increased 14 percent in 2006 [online]. Stamford: Gartner. Available from http://www.gartner.com/it/page.jsp?id=507466 [Accessed 5 November 2007].

Phua M-H \& Minowa M 2005. A GIS-based multi-criteria decision making approach to forest conservation planning at a landscape scale: A case study in the Kinabalu Area, Sabah, Malaysia. Landscape and Urban Planning 71: 207-222.

Pickup G \& Chewings VH 1996. Correlations between DEM-derived topographic indices and remotely-sensed vegetation cover in rangelands. Earth Surface Processes and Landforms 21: 517-529.

Proctor W \& Qureshi E 2005. Multi-criteria evaluation revisited [online]. Available from http://www.anzsee.org/anzsee2005papers/Proctor_Multi-criteria_evaluation.pdf [Accessed 13 May 2008].

Pummakarnchana O, Tripathi N \& Dutta J 2005. Air pollution monitoring and GIS modeling: A new use of nanotechnology based solid state gas sensors. Science and Technology of Advanced Materials 6: 251-255.

Reiger M 2006. Introduction to fruit crops. New York: Haworth Press.

Research Surveys 2006. Latest SA Web user survey results released [online]. Cape Town. Available from http://www.webchek.co.za/about press\%20saweb2006.html [Accessed 21 Aug 2006].

Reuters Foundation 2005. AlertNet [online]. Reuters. Available from http://www.alertnet.org/ [Accessed 18 Oct 2005].

Rodriguez E, Morris CS, Belz JE, Chaplin EC, Martin JM, Daffer W \& Hensley S 2005. An assessment of the SRTM topographic products. Pasadena: Jet Propulsion Laboratory.

Rossiter DG 2001. The automated land evaluation system (ALES) [online]. New York: Cornell University. Available from http://www.css.cornell.edu/landeval/ales/ales.htm [Accessed 13 May 2008].

Rossiter DG \& Van Wambeke AR 1997. Automated land evaluation system ALES version 4.65 user's manual. New York: Cornell University.

Saaty TL 1977. A scaling method for priorities in hierarchical structures. Journal of Mathematical Psychology 15: 234-281. 
Saaty TL 1998. Ranking by eigenvector versus other methods in the analytic hierarchy process. Applied Mathematics Letters 11: 212-125.

Saaty TL 2003. Decision-making with the AHP: Why is the principal eigenvector necessary. European Journal of Operational Research 145: 85-91.

Saaty TL \& Vargas LG 1991. Prediction, projection and forcasting. Boston: Kluwer Academic Publishers.

Saayman D 1981. Klimaat, grond en wingerdbougebiede. In Burger J \& Deist J (eds) Wingerdbou in Suid-Afrika, Cape Town: Maskew Miller.

Salewicza KA \& Nakayama M 2004. Development of a web-based decision support system (DSS) for managing large international rivers. Global Environmental Change 14: 25-37.

SANBI s.d. CAPE: Cape Action for People and the Environment [online]. Cape Town: SANBI. Available from http://cpu.uwc.ac.za/CAPE/index.asp\#CAPE [Accessed 13 October 2006].

SAWIS 2008. Statistics of wine-grape vines as on 30 November 2007. Paarl: SAWIS.

SAWS 2007. South Africa Weather Service background [online]. Pretoria. Available from http://www.weathersa.co.za/Corporate/History.jsp [Accessed 11 October 2007].

Schulze RE 1997. South African atlas of agrohydrology and-climatology. Report TT82/96. Pretoria: Water Research Commission.

Selvidge P 1999. How long is too long to wait for a website to load? Usability News 1.2: s.p.

Sicat RS, Carranza EJM \& Nidumolu UB 2005. Fuzzy modeling of farmers' knowledge for land suitability classification. Agricultural Systems 83: 49-75.

Smith CS, McDonald GT \& Thwaites RN 2000. TIM: Assessing the sustainability of agricultural land management. Journal of Environmental Management 60: 267-288.

Soil Survey Division Staff 1993. Soil survey manual. Washington DC: Soil Conservation Service, U.S. Department of Agriculture.

South Africa 1984. Act on conservation of agricultural resources, Act 43 of 1983. Government Gazette of South Africa 9238, 25.5.1984.

South Africa 1997. White paper on the conservation and sustainable use of South Africa's biological diversity [online]. Pretoria: Department of Environmental Affairs and Tourism. Available from http://www.environment.gov.za/PolLeg/WhitePapers/Biodiversity/Contents.htm [Accessed 16 August 2004]. 
South Africa 2000. Promotion of access to information act, Act 2 of 2000. Government Gazette of South Africa 20852.

Speight JG 1977. Landform pattern description from aerial photographs. Photogrammetria 32: 161-182.

Statistics South Africa 2001. Census 2001: Key results. Pretoria: Statistics South Africa.

Statistics South Africa 2006a. Gross domestic product. Pretoria: Statistics South Africa.

Statistics South Africa 2006b. Survey of large scale agriculture 2005. Preliminary. Pretoria: Statistics South Africa.

Statistics South Africa 2007. Mid-year population estimates, 2007 [online]. Pretoria: Statistics South Africa. Available from http://www.statssa.gov.za/publications/statsdownload.asp?PPN=P0302\&SCH=3952 [Accessed 30 June 2008].

Sun Microsystems 2008. The Source for Java Developers [online]. Available from [Accessed 1 July 2008].

Taylor DRF 2005. The theory and practice of cybercartography: An introduction. In Taylor DRF (ed) Cybercartography: theory and practice, 1-13. Amsterdam: Elsevier.

Thompson JA, Bell JC \& Butler CA 2001. Digital elevation model resolution: Effects on terrain attribute calculation and quantitative soil-landscape modeling. Geoderma 100: 67-89.

Thompson MW 1999. South African national land-cover database project: data users manual. Pretoria: CSIR.

Thwaites RN \& Slater BK 2000. Soil-landscape resource assessment for plantations - a conceptual framework towards an explicit multi-scale approach. Forest Ecology and Management 128: 123-138.

Thysen I \& Detlefsen NK 2006. Online decision support for irrigation for farmers. Agricultural Water Management 86: 269-276.

Traintaphyllou E 2000. Multi-criteria decision-making methods: A comparative study. London: Kluwer Academic.

Tsou MH 2003. An intelligent software agent architecture for distributed cartographic knowledge bases and Internet mapping services. In Peterson MP (ed) Maps and the Internet, 231-245. Amsterdam: Elsevier. 
Tsoumakas G \& Vlahavas I 1999. ISLE: An intelligent system for land evaluation. ACAI '99 Workshop on Intelligent Techniques for Spatio-Temporal Data Analysis in Environmental Applications, Chania, Greece.

Turner D 2005. Transkei and Ciskei land type survey [online]. ARC. Available from http://www.arc-iscw.agric.za/main/projects/landtype.htm [Accessed 23-06-2005].

Twery MJ, Knopp PD, Thomasma SA, Rauscher HM, Nute DE, Potter WD, Maier F, Wang J, Dass M, Uchiyama H, Glende A \& Hoffman RE 2005. NED-2: A decision support system for integrated forest ecosystem management. Computers and Electronics in Agriculture 49: 2443.

U.S. Department of Health and Human Services 2006. Research-based Web design \& usability guidelines [online]. Available from http://www.usability.gov/pdfs/guidelines.html [Accessed 6 September 2007].

U.S. Department of Labor 2006. Career voyages [online]. U.S. Department of Labor. Available from http://www.careervoyages.gov/index.cfm [Accessed 9 February 2006].

Vahidov R \& Kersten GE 2004. Decision station: situating decision support systems. Decision Support Systems 38: 283-303.

Valentini N, Me G, Spanna F \& Lovisetto M 2004. Chilling and heat requirement in apricot and peach varieties. Acta Horticulturae 636: 199-203.

Van der Merwe JH 1997. GIS-aided land evaluation and decision-making for regulating urban expansion: A South African case study. GeoJournal 43: 135-151.

Van der Merwe JH 2006. Multi-criteria evaluation for land planning: Urban application. Presentation given to the Department of Environmental Affairs and Development Planning, Provincial Government of the Western Cape, Stellenbosch, Stellenbosch University.

Van der Merwe JH, Ferreira SLA \& Van Niekerk A 2008. A spatial gap-analysis of tourism development opportunity in the Western Cape Province. Report 01/2008. Stellenbosch: Stellenbosch University.

Van der Merwe JH \& Steyl I 2005. Rural solid waste management: A planning strategy for higher density agricultural regions. Journal of Public Administration 40: 295-313.

Van der Merwe JH \& Von Holdt DS 2006. Environmental footprint of aircraft noise exposure at Cape Town International Airport. The South African Geographical Journal 88: 177-193.

Van Niekerk A 1997. Die ontwikkeling van geografiese inligtingstelsels vir omgewingsbestuur in die Wes-Kaap. MSc thesis. Stellenbosch: Stellenbosch University. 
Van Niekerk A 2001. Western Cape Digital Elevation Model: Product description. Stellenbosch: Centre for Geographical Analysis, Stellenbosch University.

Van Niekerk A \& Schloms BHA 2001. Automated mapping of land components from elevation data. Paper presented at the Fourth Biennial International Conference of the Society of South African Geographers, Rawsonville.

Van Niekerk A \& Schloms BHA 2002. A comparison of automatically mapped land components with large-scale soil maps. Paper presented at the Regional Conference of the International Geographical Union, Durban.

Van Ranst E, Tang H, Groenemans R \& Sinthurahat S 1996. Application of fuzzy logic to land suitability for rubber production in peninsular Thailand. Geoderma 70: 1-19.

Van Wyngaarden R \& Waters N 2007. An unfinished revolution - gaining perspective on the future of GIS. GeoWorld September: s.p.

Varma VK, Ferguson I \& Wild I 2000. Decision support system for the sustainable forest management. Forest Ecology and Management 128: 49-55.

Vreeker R, Nijkamp P \& Ter Welle C 2002. A multicriteria decision support methodology for evaluating airport expansion plans. Transportation Research 7: 27-47.

W3C 2002. XHTML 1.0 The Extensible HyperText Markup Language (Second Edition) [online]. Available from http://www.w3.org/TR/xhtml1/ [Accessed 1 July 2008].

W3C 2006. Extensible Markup Language (XML) 1.1 (Second Edition) [online]. Available from http://www.w3.org/TR/xml11/ [Accessed 1 July 2008].

W3C 2008. HTML 5 [online]. Available from http://www.w3.org/html/wg/html5/ [Accessed 4 April 2008].

W3Schools 2008a. DHTML is the art of combining HTML, JavaScript, DOM, and CSS. [online]. Available from [Accessed 1 July 2008].

W3Schools 2008b. SQL join [online]. Available from

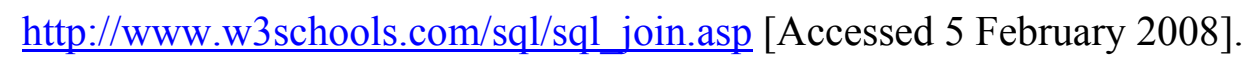

Walther BA, Schaffer N, Van Niekerk A, Thuiller W, Rahbek C \& Chown SL 2007. Modelling the winter distribution of a rare and endangered migrant, the Aquatic Warbler Acrocephalus paludicola. Ibis 149: 701-714. 
Wandahwa P \& Van Ranst E 1996. Qualitative land suitability assessment for pyrethrum cultivation in west Kenya based upon computer-captured expert knowledge and GIS. Agriculture, Ecosystems \& Environment 56: 187-202.

Wang F, Hall GB \& Subaryono 1990. Fuzzy information representation and processing in conventional GIS software: Database design and application. International Journal of Geographical Information Systems 4: 261-283.

Wang K-J \& Chein C-F 2003. Designing an Internet-based group decision support system. Robotics and Computer Integrated Manufacturing 19: 65-77.

Wang WK 2005. A knowledge-based decision support system for measuring the performance of government real estate investment. Expert Systems with Applications 29: 901-912.

Wikipedia 2005. Internet [online]. Wikimedia Foundation Inc. Available from http://en.wikipedia.org/wiki/Internet [Accessed 18 Oct 2005].

Wood J 2006. Landserf [online]. London: City University. Available from http://www.landserf.org/ [Accessed 16 February 2006].

Wood LJ \& Dragicevic S 2007. GIS-based multicriteria evaluation and fuzzy sets to identify priority sites for marine protection. Biodiversity and Conservation 16: 2539-2558.

Yalcin G \& Akyurek Z 2004. Analysing flood vulnerable areas with multicriteria evaluation [online]. Available from http://cartesia.org/geodoc/isprs2004/comm2/papers/154.pdf [Accessed 13 May 2008].

Zadeh L 1965. Fuzzy sets. Information and Control 8: 338-353.

Zhou Q \& Liu X 2004. Analysis of errors of derived slope and aspect related to DEM data properties. Computers \& Geosciences 30: 369-378. 


\section{PERSONAL COMMUNICATIONS}

Moss D 2006. Interview about land evaluation systems. Stellenbosch, Dennis Moss Partnership (11 November 2006).

Schloms BHAS 2007. Interview about soil science and pedogenesis. Stellenbosch, Stellenbosch University (18 September 2007).

Schloms BHAS 2007. Interview about the environmental requirements of perennial crops. Stellenbosch, Stellenbosch University (29 February 2008).

Van der Merwe A (ajvdmerw@pgwc.gov.za) 2008. RE: ArcIMS. Email to A van Niekerk (avn@sun.ac.za) (7 July 2008).

Van der Merwe JH 2008. Discussion on AHP process. Stellenbosch, Stellenbosch University (30 April 2008). 


\section{APPENDICES}

Appendix A Avenue script to extract soil effective depth, clay content in the A 203 horizon and mechanical limitations from the land type data

Appendix B $\quad$ Automatic Land Component Mapper (ALCoM) Avenue script

Appendix C Avenue script to extract land property data from raster datasets for each land unit in the land unit database

Appendix D $\quad$ Field and integrity rule descriptions for each entity in the knowledge 216 base

Appendix E Visual Basic procedure to calculate each land unit's suitability based 221 on the land use requirements in the knowledge base

Appendix F ArcIMS map configuration file

Appendix G CD containing the CLUES website source code 


\section{APPENDIX A}

Avenue script to extract soil effective depth, clay content in the A horizon and mechanical limitations from the land type data

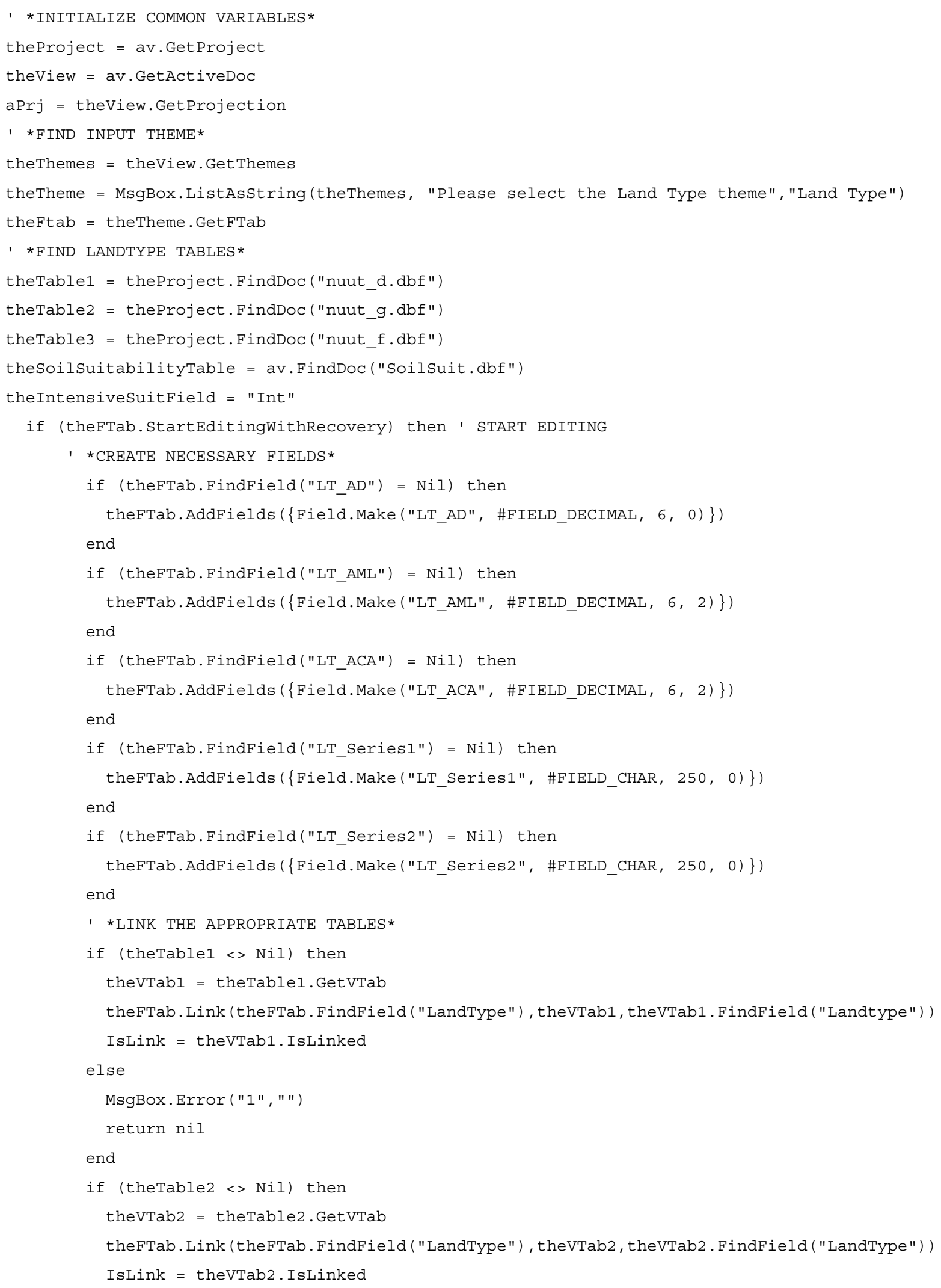


else

MsgBox.Error ("2", " ")

return nil

end

theselection $=$ theFTab. GetSelection

theselection. SetAll

numberofunits $=$ theselection. Count

' *GET INFORMATION FOR EACH LAND TYPE*

for each rec in 0.. (numberofunits - 1) ' FOR EACH LANDTYPE

if (theFTab.ReturnValue (theFTab.FindField("LandType"), rec) <> " ") then

' *SELECT CURRENT LAND TYPE*

theSelection.ClearAll

theselection. Set ( $r e c$ )

theFTab.SetSelection (theselection)

theselection2 = theVTab1. GetSelection

theselection $3=$ theVTab2.GetSelection

AverageDepth $=0$

AverageSoilscore $=0$

AverageMechanicalLimitation $=0$

MechanicalLimitation $=0$

Totalseries $=$ " "

Depth $=0$

AverageClayA $=0$

Clay $=0$

theSoilsortList $=\{\}$

thesoilsortPercList $=\{\}$

' *FIND LIST OF TERRAIN TYPES ASSOCIATED WITH THIS LAND TYPE*

for each rec2 in theselection2 ' FOR EACH TERRAIN TYPE

' *TEMPORARILY STORE TERRAIN INFORMATION*

thePercentageofTerrain $=$

theVTab1.ReturnValue (theVTab1.FindField ("Terrain_p"), rec2)

terrain = theVTab1.ReturnValue (theVTab1.FindField("Terrain_u"), rec2)

AverageSoilscoreofTerrainType $=0$

AverageMechanicalLimitationofTerrainType $=0$

AverageDepthofTerrainType $=0$

AverageClayAofTerrainType $=0$

TotalSeriesofTerrainType = " "

' *GET LIST OF SOIL TYPES ASSOCIATED WITH EACH TERRAIN TYPE*

for each rec3 in theselection3, FOR EACH SOIL SERIES

thePercofSoil = theVTab2.ReturnValue (theVTab2.FindField ("Soil_p"), rec3)

terrain2 = theVTab2.ReturnValue (theVTab2.FindField("Terrain_u"), rec3)

if (terrain = terrain2) then, LOOK FOR CURRENT TERRAIN TYPE IN SOIL TABLE

' *GET AVERAGE SOIL DEPTH ASSOCIATED WITH EACH SOIL IN THE CURRENT TERRAIN TYPE*

if (theVTab2.ReturnValue (theVTab2.FindField("Soil_d_t"), rec3) = "-") then

Depth = theVTab2.ReturnValue (theVTab2.FindField("Soil_d_u"), rec3) +

( (theVTab2.ReturnValue (theVTab2.FindField ("Soil_d_l"), rec3) -

theVTab2.ReturnValue (theVTab2.FindField ("Soil_d_u"), rec3)) / 2)

elseif (theVTab2.ReturnValue (theVTab2.FindField("Soil_d_t"), rec3) = "<")

then

Depth $=$ theVTab2.ReturnValue (theVTab2.FindField("Soil_d_l"), rec3) / 2

elseif (theVTab2.ReturnValue (theVTab2.FindField("Soil_d_t"), rec3) = ">")

then 


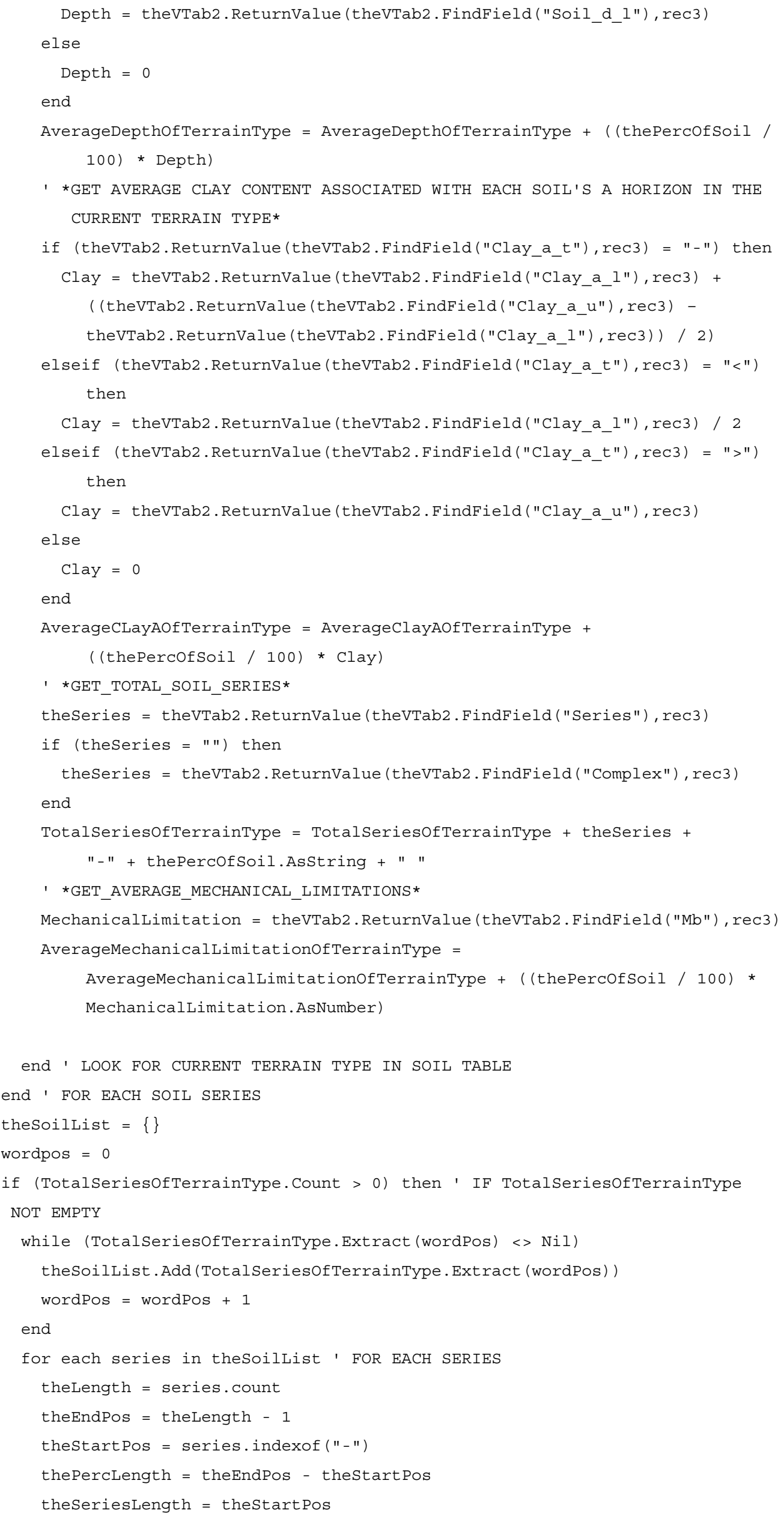




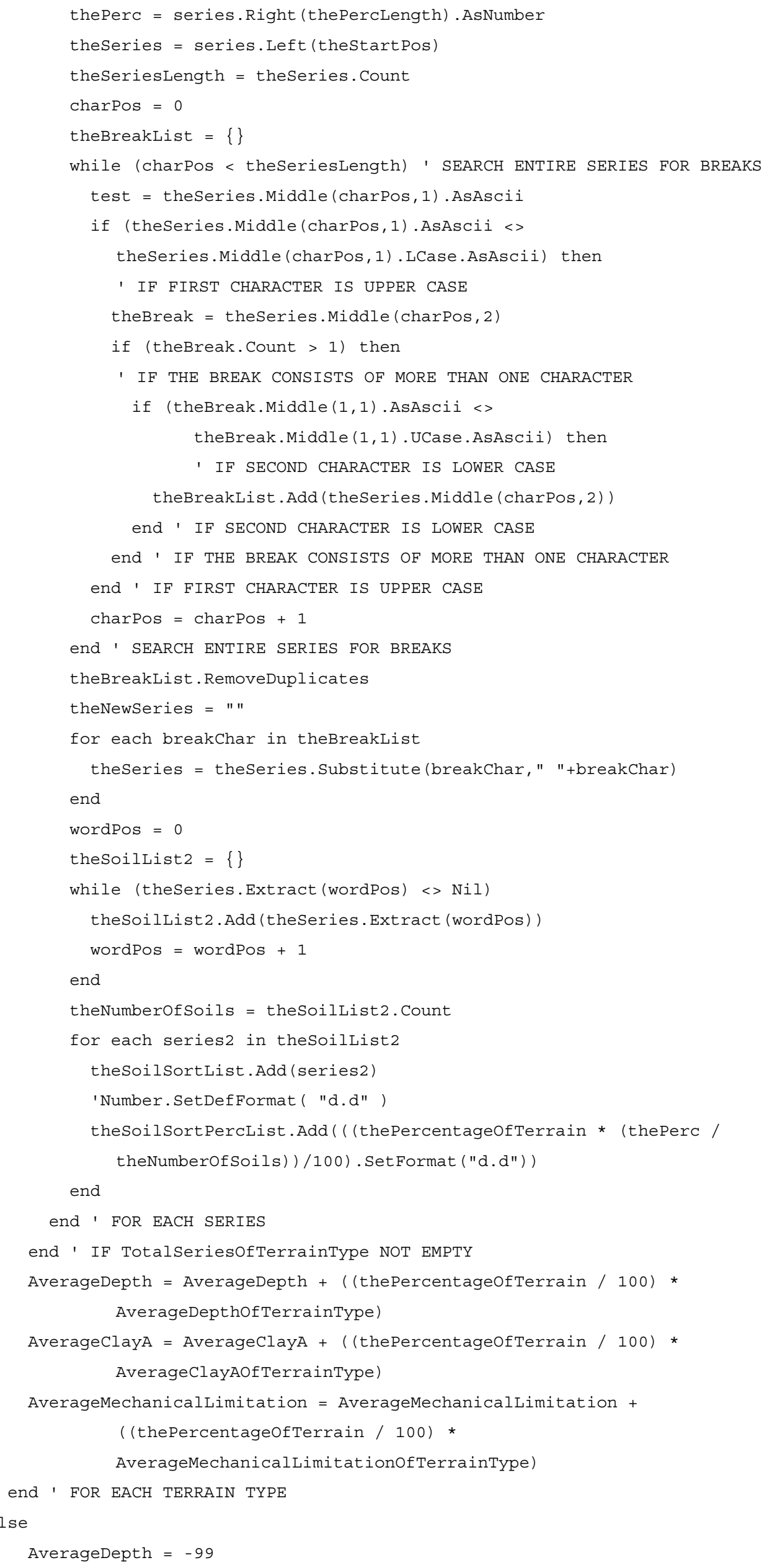




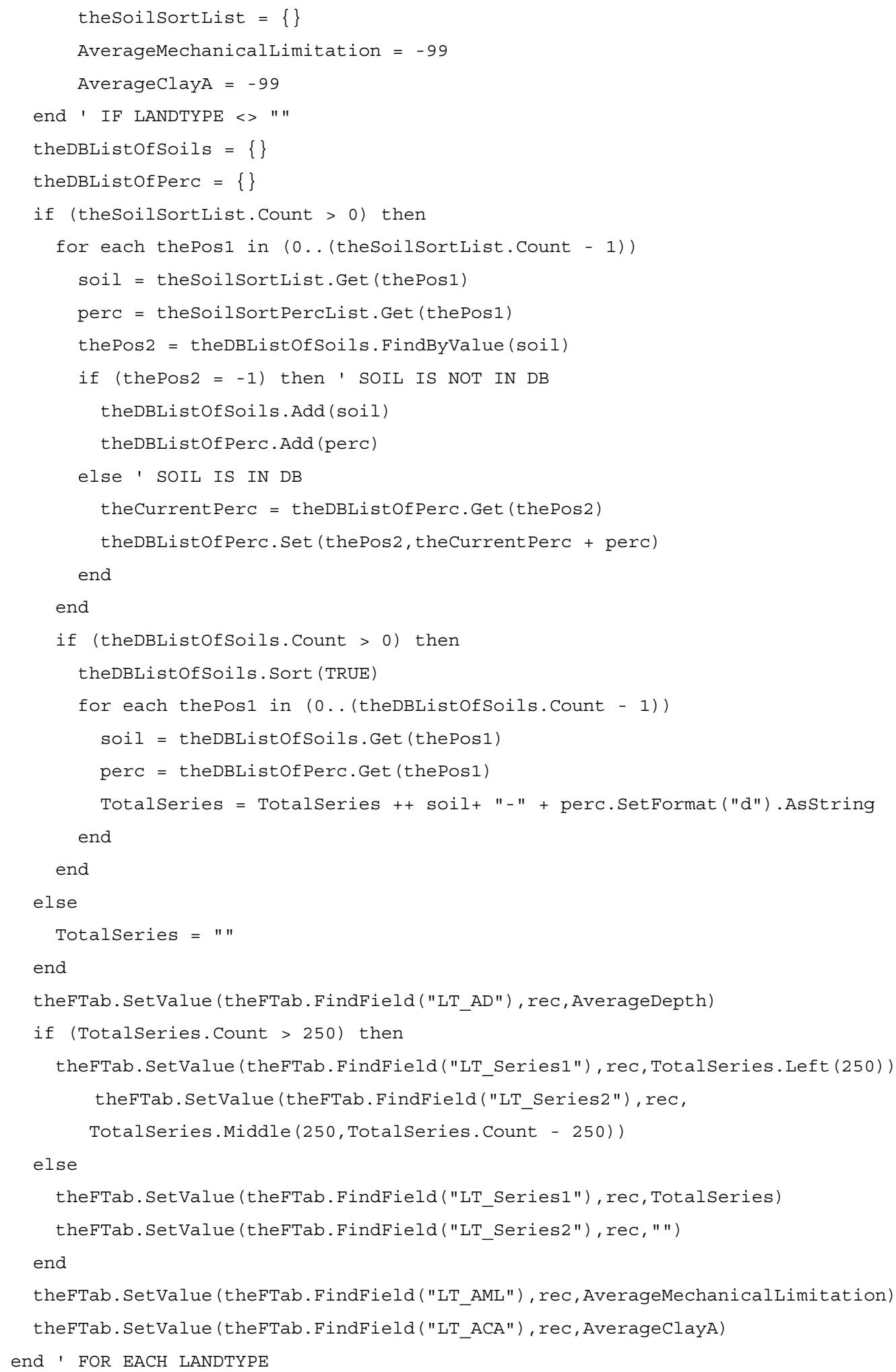




\section{APPENDIX B}

\section{Automatic Land Component Mapper (ALCoM) Avenue script}

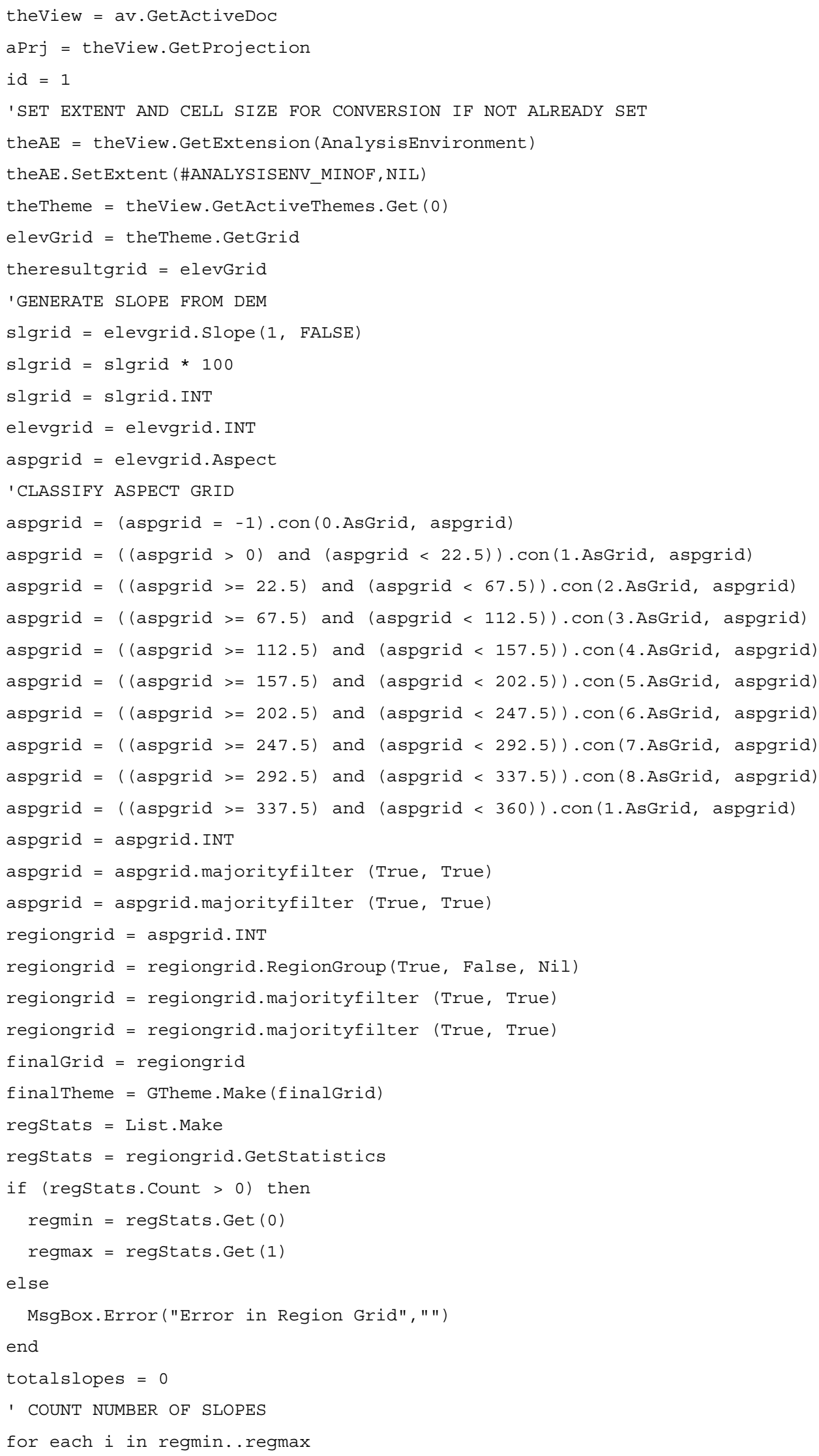




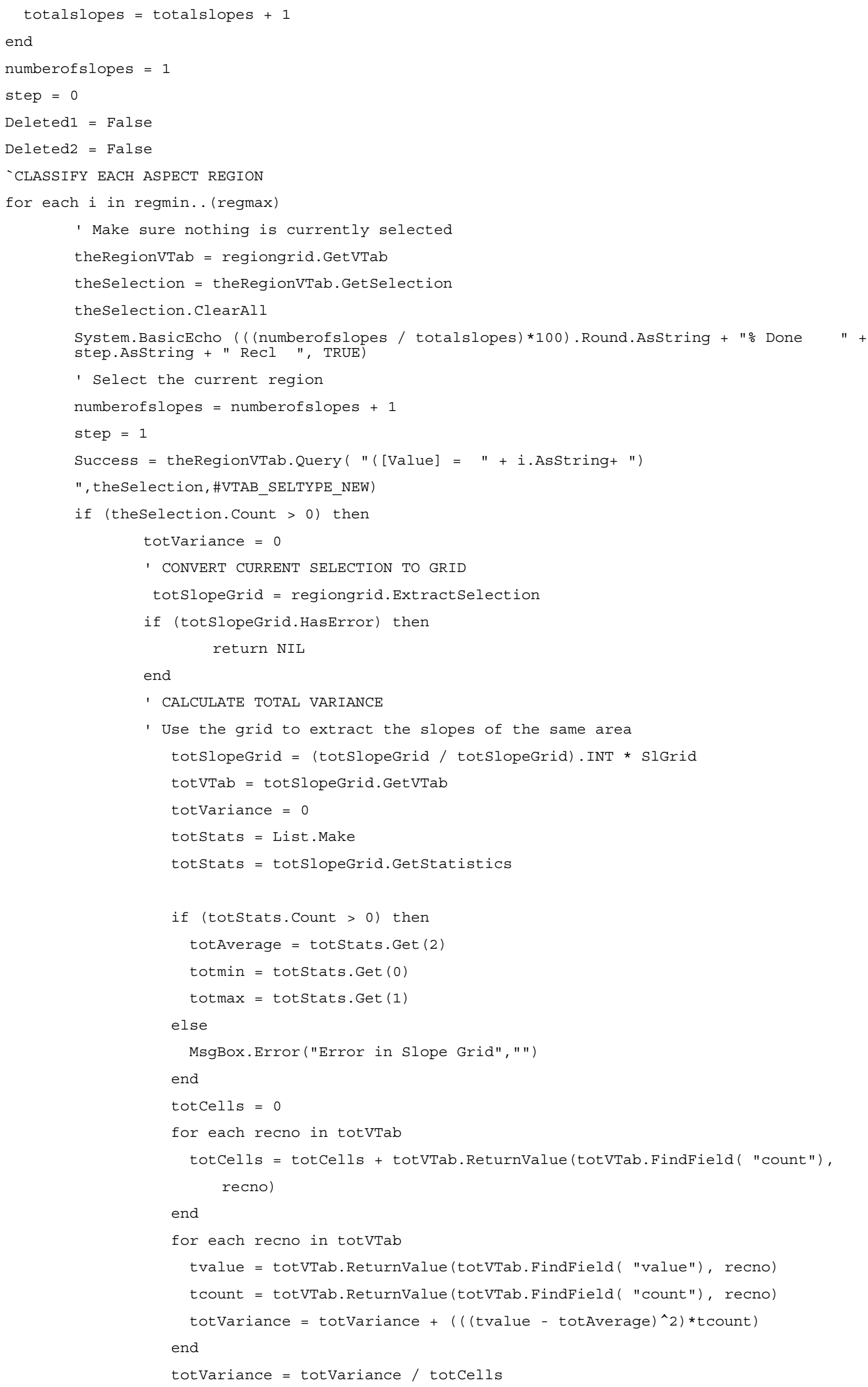


' Decide whether the area should be reclassified

if ((totCells > 50) and ((totmax - totmin) > 1) and (totVariance > 1)) then ' CREATE NEW LEGEND FOR SPOPE GRID USING NATURAL BREAKS

theSlopeTheme $=$ GTheme.Make (totSlopeGrid)

theslopeLegend $=$ theSlopeTheme. GetLegend

theslopeLegend.Natural (theslopeTheme, "Value", 2)

' STORE GRID ACCORDING TO ITS NEW LEGEND

theField $=$

theSlopelegend. GetFieldNames.Get (theslopeLegend. GetFieldNames. Count - 1)

aClasslist $=$ theSlopeLegend.GetClassifications

' the list of classifications and each classification needs to

' be cloned so that the labels in the original legend do not change theClassList $=$ aClassList $\cdot$ Deepclone

numberofclasses $=0$

for each $C$ in theclasslist

numberofclasses $=$ numberofclasses +1

end

count $=1$

- Add labels for each classification starting at 1

for each $\mathrm{C}$ in theclasslist

if (count < numberofclasses) then

c. SetLabel (count. Asstring)

count $=$ count +1

end

end

theResultGrid $=$

totSlopeGrid. ReclassByClassList (theField, theClassList, FALSE)

avList $=$ List. Make

noc $=2$

count $=1$

$\mathrm{DO}=$ true

IsSmaller $=$ true

' While the variance is high, reclassify

While (DO)

theResultVTab $=$ theResultGrid.GetVTab

theSelection $=$ theResultVTab.GetSelection

theselection. ClearAll

Success $=$ theResultVTab.Query $($ "[Value $]="$ + count.AsString+ "

", theselection, \#VTAB_SELTYPE_NEW)

if (theselection. Count > 0) then

CurrentGrid = theResultGrid.Extractselection

CurrentGrid = (CurrentGrid / CurrentGrid). INT

CurrentGrid = CurrentGrid * SlGrid

CurrentVTab $=$ CurrentGrid.GetVTab

Currentstats $=$ List. Make

CurrentStats $=$ CurrentGrid.GetStatistics

if (Currentstats. Count > 0) then

CurrentAverage $=$ CurrentStats.Get (2)

else

MsgBox.Error("Error in Current Grid"," ")

end 


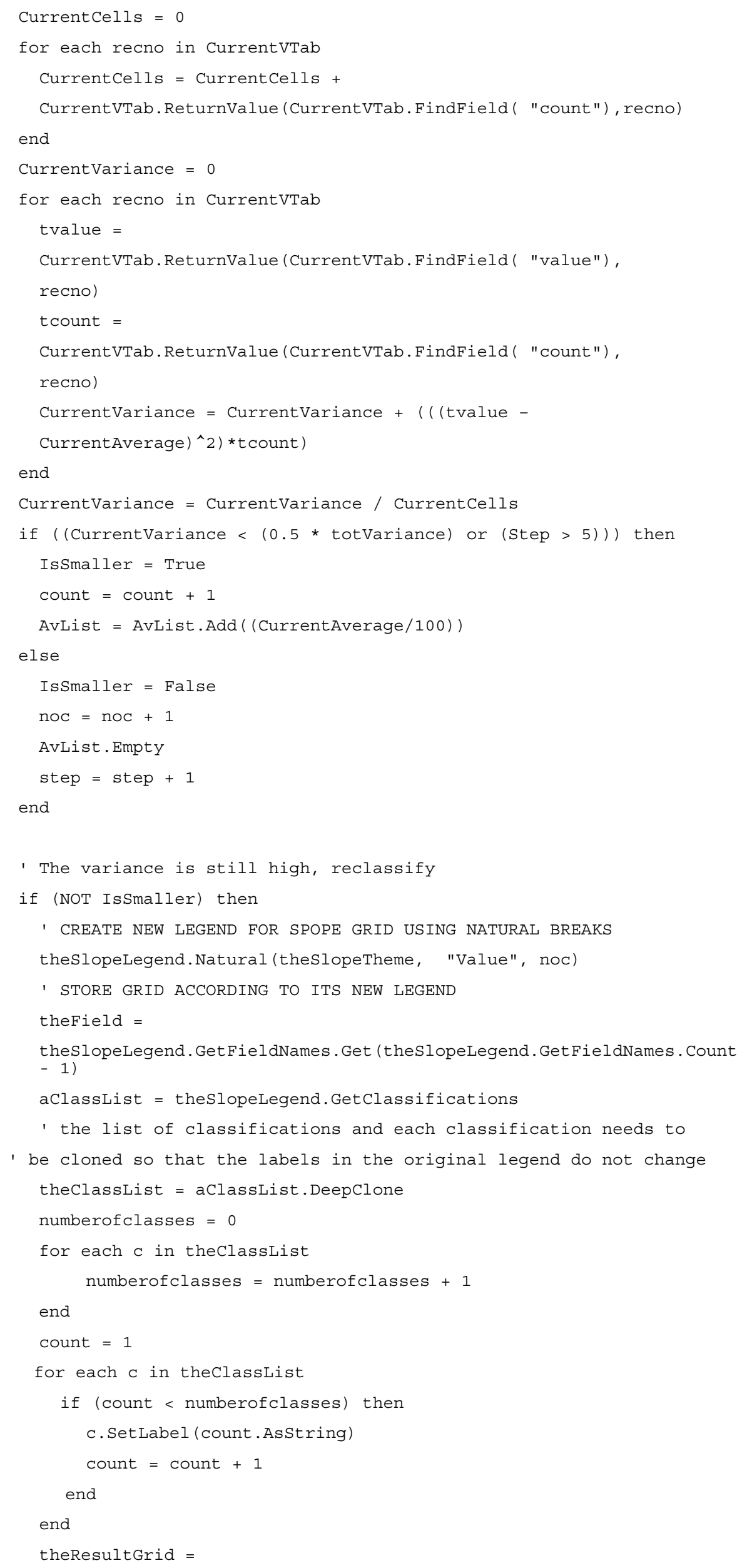


totSlopeGrid.ReclassByClassList (theField, theClassList, FALSE)

count $=1$

end

else

Do $=$ False

' STORE GRID ACCORDING TO ITS NEW LEGEND

ResultTheme $=$ GTheme. Make (theResultGrid)

theResultLegend $=$ ResultTheme.GetLegend

theField = theResultLegend.GetFieldNames.Get

(theResultLegend.GetFieldNames. Count - 1)

aclasslist $=$ theResultLegend.GetClassifications

' the list of classifications and each classification needs to

' be cloned so that the labels in the original legend do not change theClassList $=$ aclassList $\cdot$. DeepClone

numberofclasses $=0$

for each $\mathrm{C}$ in theclassList

numberofclasses $=$ numberofclasses +1

end

count $=1$

for each $c$ in theclasslist

if (count $<=$ numberofclasses) then

c.SetLabel (AvList. Get (count - 1). Round.AsString)

count $=$ count +1

end

end

theResultGrid $=$

theResultGrid.ReclassByClassList (theField, theClassList, FALSE)

end

end

theAE = theView.GetExtension (AnalysisEnvironment)

theAE.SetExtent (\#ANALYSISENV_VALUE, elevgrid.GetExtent)

finalgrid $=$ (theResultGrid. IsNull). Con (finalgrid, theResultGrid)

if (finalgrid.HasError) then return NIL end

FinalTheme $=$ GTheme $\cdot$ Make $($ finalgrid $)$

else

theAE = theView.GetExtension (AnalysisEnvironment)

theAE.SetExtent (\#ANALYSISENV_VALUE, elevgrid.GetExtent)

finalgrid $=$

(totSlopeGrid.IsNull). Con (finalgrid, (totAverage/100).AsGrid.INT)

if (finalgrid.HasError) then return NIL end

FinalTheme $=$ GTheme. Make (finalgrid)

end

end

end

If (finalgrid.HasError) then

return NIL

else

theView. AddTheme (Final Theme)

end 


\section{APPENDIX C}

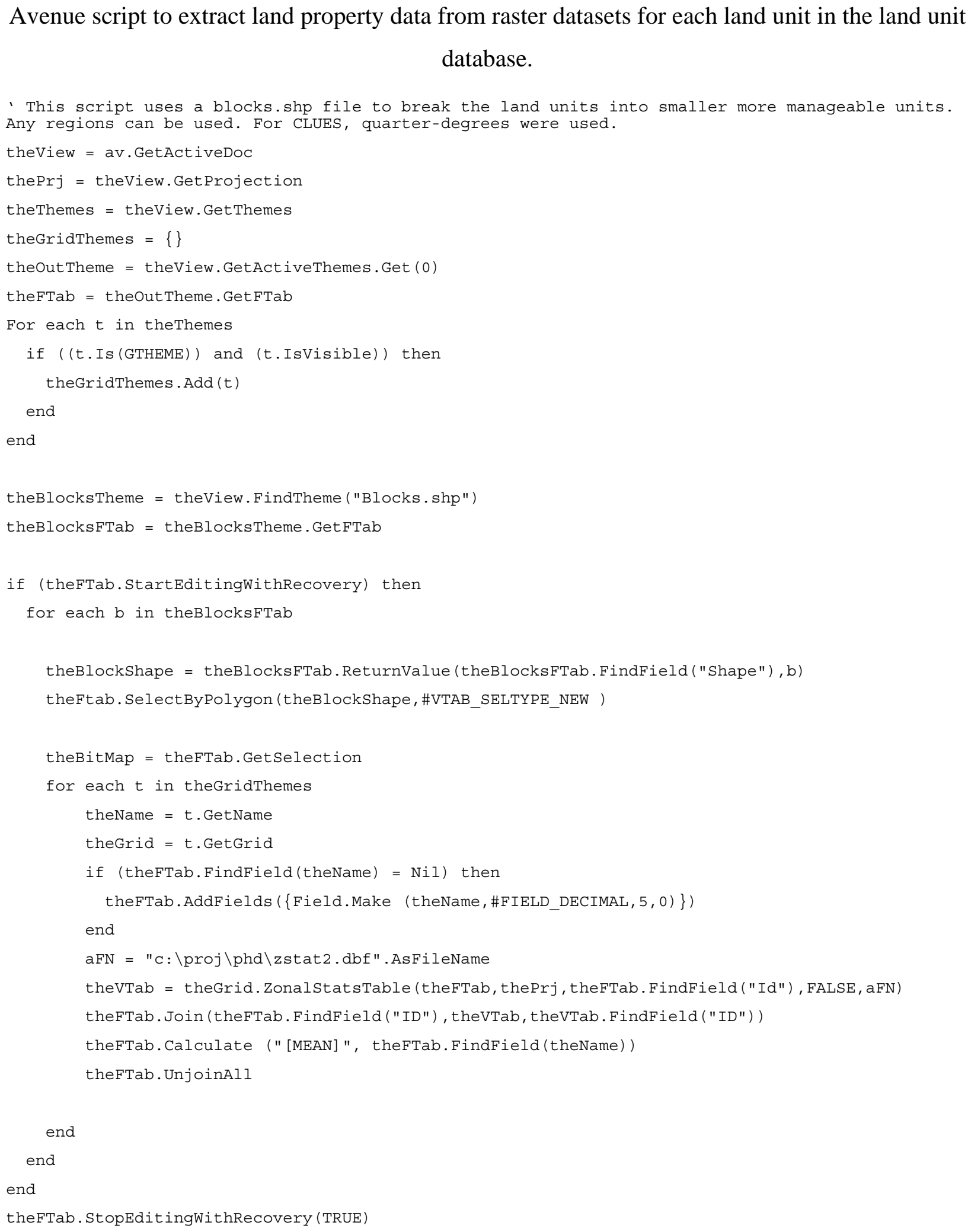




\section{APPENDIX D}

Field and integrity rule descriptions for each entity in the knowledge base

\begin{tabular}{|l|l|}
\hline \multicolumn{2}{|l|}{ ENTITY: LAND USE } \\
\hline ATTRIBUTE & DOMAIN AND TRIGGER RULES \\
\hline LAND_USE_ID & $\begin{array}{l}\text { Data type: number } \\
\text { Format: integer } \\
\text { Uniqueness: unique } \\
\text { Null support: non-null } \\
\text { Insert trigger: none } \\
\text { Update trigger: not allowed } \\
\text { Delete trigger: ensure no child entities are present }\end{array}$ \\
\hline USER_ID & $\begin{array}{l}\text { See USER entity } \\
\text { NAME }\end{array}$ \\
\hline $\begin{array}{l}\text { Data type: text } \\
\text { Length: } 100 \\
\text { Uniqueness: non-unique } \\
\text { Null support: non-null }\end{array}$ \\
\hline
\end{tabular}

\section{ENTITY: LAND REQUIREMENT}

\begin{tabular}{|l|l|}
\hline ATTRIBUTE & DOMAIN AND TRIGGER RULES \\
\hline LAND_REQUIREMENT_ID & $\begin{array}{l}\text { Data type: number } \\
\text { Format: integer } \\
\text { Uniqueness: unique } \\
\text { Null support: non-null } \\
\text { Insert trigger: none } \\
\text { Update trigger: not allowed } \\
\text { Delete trigger: ensure no child entities are present }\end{array}$ \\
\hline LAND_USE_ID & See LAND_USE entity \\
\hline LAND_PROPERTY_ID & See LAND_PROPERTY entity \\
\hline WEIGHT & $\begin{array}{l}\text { Data type: number } \\
\text { Length: } 4 \text { digits } \\
\text { Format: decimal (two decimal places) } \\
\text { Uniqueness: non-unique } \\
\text { Null support: non-null }\end{array}$ \\
\hline
\end{tabular}




\begin{tabular}{|c|c|}
\hline \multicolumn{2}{|c|}{ ENTITY: LAND_REQUIREMENT_RULE } \\
\hline ATTRIBUTE & DOMAIN AND TRIGGER RULES \\
\hline \multirow[t]{7}{*}{ LAND_REQUIREMENT_RULE_ID } & Data type: number \\
\hline & Format: integer \\
\hline & Uniqueness: unique \\
\hline & Null support: non-null \\
\hline & Insert trigger: none \\
\hline & Update trigger: not allowed \\
\hline & Delete trigger: ensure no child entities are present \\
\hline LAND_REQUIREMENT_ID & See LAND_REQUIREMENT entity \\
\hline \multirow[t]{5}{*}{ SUITABILITY } & Data type: text \\
\hline & Length: 2 characters \\
\hline & Format: alphanumeric (options: N2, N1, S3, S2, S1) \\
\hline & Uniqueness: non-unique \\
\hline & Null support: non-null \\
\hline \multirow[t]{4}{*}{ LOWER_VALUE } & Data type: number \\
\hline & Format: decimal (3 places) \\
\hline & Uniqueness: non-unique \\
\hline & Null support: non-null \\
\hline \multirow[t]{4}{*}{ MIDDLE_VALUE } & Data type: number \\
\hline & Format: decimal (3 places) \\
\hline & Uniqueness: non-unique \\
\hline & Null support: non-null \\
\hline \multirow[t]{4}{*}{ UPPER_VALUE } & Data type: number \\
\hline & Format: decimal (3 places) \\
\hline & Uniqueness: non-unique \\
\hline & Null support: non-null \\
\hline \multirow[t]{6}{*}{ CURVE_ID } & Data type: number \\
\hline & Length: 1 \\
\hline & Range: $1-2$ \\
\hline & Format: integer \\
\hline & Uniqueness: non-unique \\
\hline & Null support: non-null \\
\hline
\end{tabular}

\section{ENTITY: LAND_PROPERTY}

\begin{tabular}{|l|l|}
\hline ATTRIBUTE & DOMAIN AND TRIGGER RULES \\
\hline LAND_PROPERTY_ID & Data type: number \\
& Format: integer \\
& Uniqueness: unique \\
& Null support: non-null \\
& Insert trigger: none \\
\hline
\end{tabular}




\begin{tabular}{|c|c|}
\hline & $\begin{array}{l}\text { Update trigger: not allowed } \\
\text { Delete trigger: ensure no child entities are present }\end{array}$ \\
\hline DATA_SOURCE_ID & See DATA_SOURCE entity \\
\hline NAME & $\begin{array}{l}\text { Data type: text } \\
\text { Length: } 50 \\
\text { Uniqueness: non-unique } \\
\text { Null support: non-null }\end{array}$ \\
\hline UNIT & $\begin{array}{l}\text { Data type: text } \\
\text { Length: } 50 \\
\text { Uniqueness: non-unique } \\
\text { Null support: non-null }\end{array}$ \\
\hline MIN & $\begin{array}{l}\text { Data type: number } \\
\text { Format: decimal (3 places) } \\
\text { Uniqueness: non-unique } \\
\text { Null support: non-null }\end{array}$ \\
\hline MAX & $\begin{array}{l}\text { Data type: number } \\
\text { Format: decimal (3 places) } \\
\text { Uniqueness: non-unique } \\
\text { Null support: non-null }\end{array}$ \\
\hline
\end{tabular}

\section{ENTITY: LAND_UNIT}

\begin{tabular}{|c|c|}
\hline ATTRIBUTE & DOMAIN AND TRIGGER RULES \\
\hline LAND_UNIT_ID & $\begin{array}{l}\text { Data type: number } \\
\text { Format: integer } \\
\text { Uniqueness: unique } \\
\text { Null support: non-null } \\
\text { Insert trigger: none } \\
\text { Update trigger: not allowed } \\
\text { Delete trigger: ensure no child entities are present }\end{array}$ \\
\hline LAND_PROPERTY_ID & See LAND_PROPERTY entity \\
\hline VALUE & $\begin{array}{l}\text { Data type: numeric } \\
\text { Length: } 10 \text { digits } \\
\text { Range: } 0.000-9999999.000 \\
\text { Format: decimal (three decimal places) } \\
\text { Uniqueness: non-unique } \\
\text { Null support: non-null }\end{array}$ \\
\hline
\end{tabular}

\section{ENTITY: PROJECT}

\begin{tabular}{|l|l|}
\hline ATTRIBUTE & DOMAIN AND TRIGGER RULES \\
\hline PROJECT_ID & $\begin{array}{l}\text { Data type: number } \\
\text { Format: integer }\end{array}$ \\
\hline
\end{tabular}




\begin{tabular}{|c|c|}
\hline & $\begin{array}{l}\text { Uniqueness: unique } \\
\text { Null support: non-null } \\
\text { Insert trigger: none } \\
\text { Update trigger: not allowed } \\
\text { Delete trigger: ensure no child entities are present }\end{array}$ \\
\hline USER_ID & See USER entity \\
\hline LAND_USE_ID & See LAND_USE entity \\
\hline NAME & $\begin{array}{l}\text { Data type: text } \\
\text { Length: } 50 \\
\text { Uniqueness: non-unique } \\
\text { Null support: non-null }\end{array}$ \\
\hline MODIFIED & $\begin{array}{l}\text { Data type: text } \\
\text { Length: } 50 \\
\text { Uniqueness: non-unique } \\
\text { Null support: non-null }\end{array}$ \\
\hline MIN_X & $\begin{array}{l}\text { Data type: number } \\
\text { Format: integer } \\
\text { Uniqueness: non-unique } \\
\text { Null support: non-null }\end{array}$ \\
\hline MAX_X & $\begin{array}{l}\text { Data type: number } \\
\text { Format: integer } \\
\text { Uniqueness: non-unique } \\
\text { Null support: non-null }\end{array}$ \\
\hline MIN_Y & $\begin{array}{l}\text { Data type: number } \\
\text { Format: integer } \\
\text { Uniqueness: non-unique } \\
\text { Null support: non-null }\end{array}$ \\
\hline MAX_Y & $\begin{array}{l}\text { Data type: number } \\
\text { Format: integer } \\
\text { Uniqueness: non-unique } \\
\text { Null support: non-null }\end{array}$ \\
\hline
\end{tabular}

\section{ENTITY: DATA_SOURCE}

\begin{tabular}{|l|l|}
\hline ATTRIBUTE & DOMAIN AND TRIGGER RULES \\
\hline DATA_SOURCE_ID & Data type: number \\
& Format: integer \\
& Uniqueness: unique \\
& Null support: non-null \\
& Insert trigger: none \\
& Update trigger: not allowed \\
& Delete trigger: ensure no child entities are present \\
\hline NAME & Data type: text \\
\hline
\end{tabular}




\begin{tabular}{|l|l|}
\hline SCALE & $\begin{array}{l}\text { Length: } 50 \text { characters } \\
\text { Uniqueness: non-unique } \\
\text { Null support: non-null }\end{array}$ \\
\hline ORIGIN & $\begin{array}{l}\text { Data type: text } \\
\text { Length: } 50 \text { characters } \\
\text { Uniqueness: non-unique } \\
\text { Null support: non-null }\end{array}$ \\
\hline $\begin{array}{l}\text { Data type: text } \\
\text { Length: } 50 \text { characters } \\
\text { Uniqueness: non-unique } \\
\text { Null support: non-null }\end{array}$ \\
\hline
\end{tabular}




\section{APPENDIX E}

Visual Basic procedure to calculate each land unit’s suitability based on the land use requirements in the knowledge base

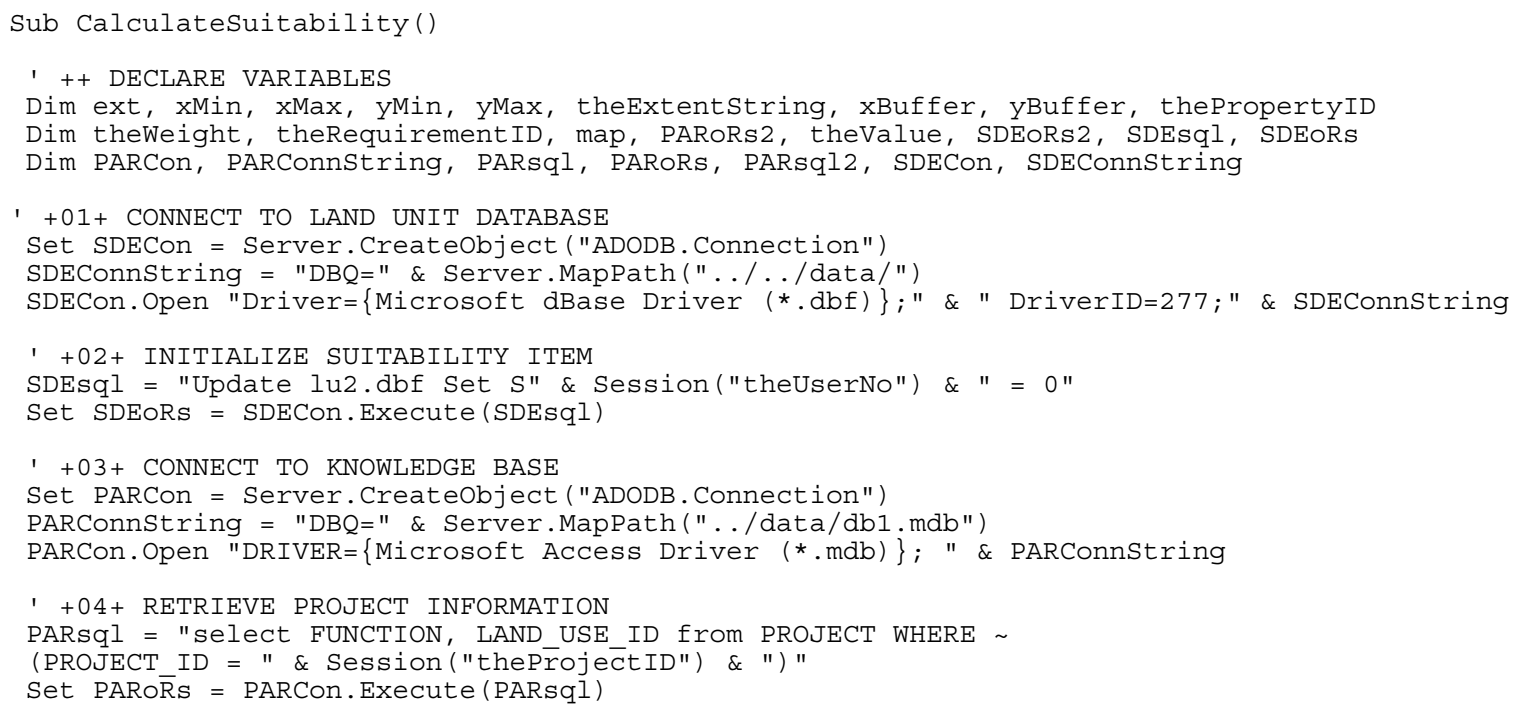




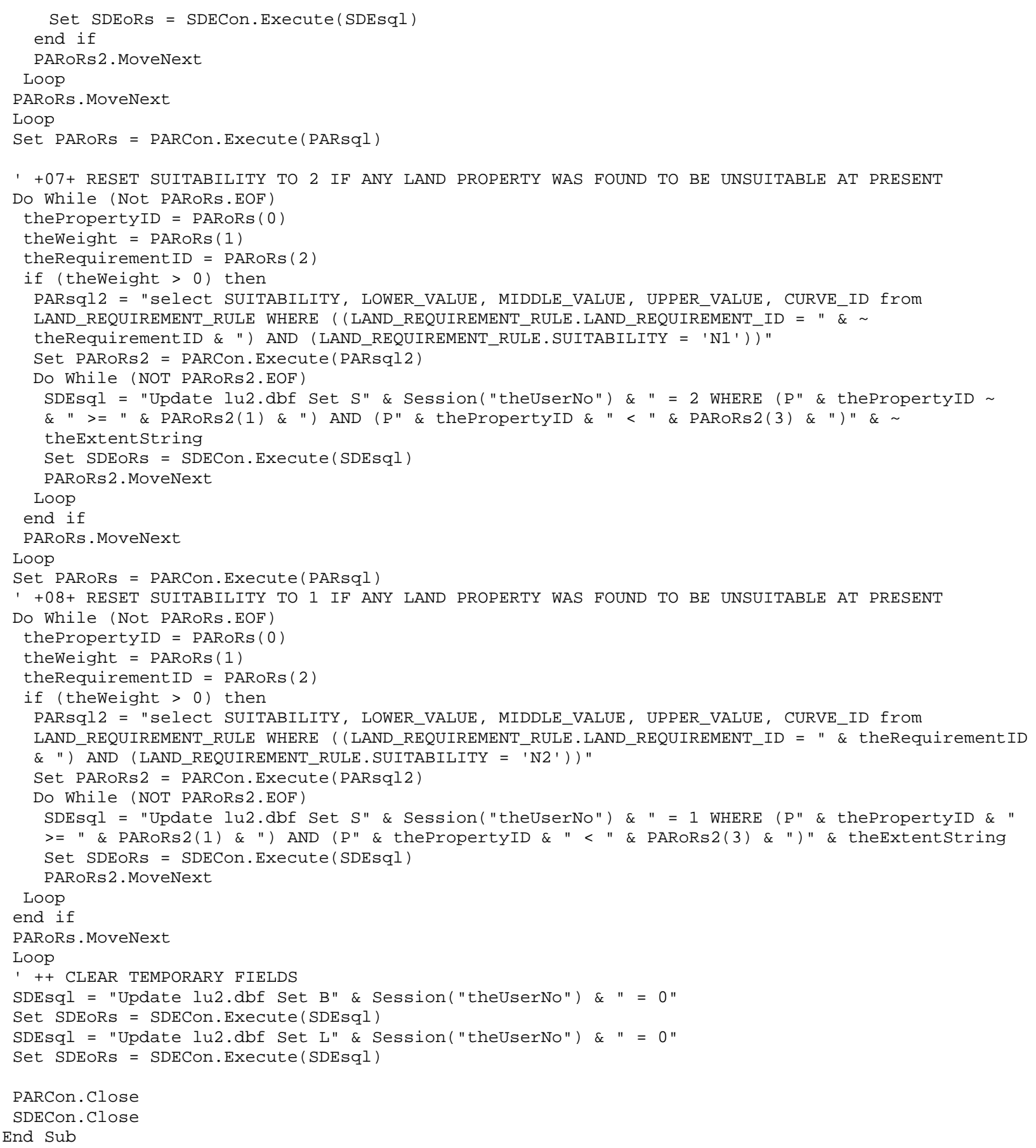




\section{APPENDIX F}

\section{ArcIMS map configuration file}

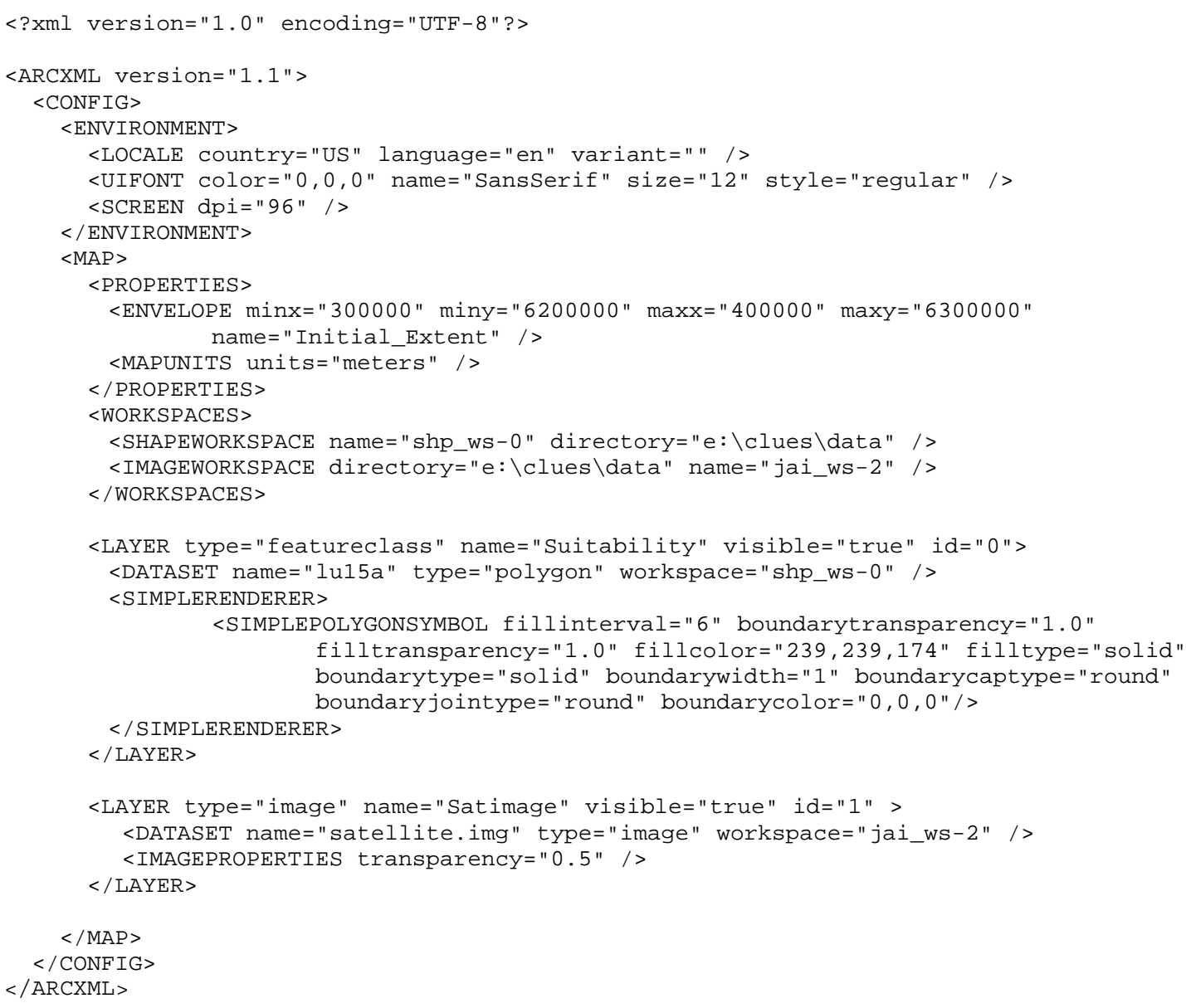


APPENDIX G

CD containing the CLUES website source code (see back cover) 